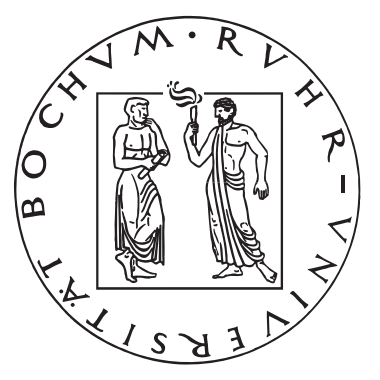

\title{
Bruchenergie laufender Risse \\ in Gestein
}

Dissertation

zur

Erlangung des Grades

Doktor-Ingenieur

der

Fakultät für Bau- und Umweltingenieurwissenschaften der Ruhr-Universität Bochum

von

Andreas Bertram

aus Bochum

Bochum, im Mai 2008 



\section{Vorwort}

Die vorliegende Arbeit entstand während meiner Tätigkeit als wissenschaftlicher Mitarbeiter am Institut für Mechanik der Ruhr-Universität Bochum, Arbeitsgruppe für Experimentelle Mechanik. Sie wurde im Rahmen des Sonderforschungsbereiches 526 Rheologie der Erde von der Deutschen Forschungsgemeinschaft gefördert und von der Fakultät für Bau- und Umweltingenieurwissenschaften der Ruhr-Universität Bochum als Dissertation angenommen.

Herrn Prof. Dr.-Ing. J. F. Kalthoff danke ich herzlich für die Anregung und Betreuung dieser Arbeit. Durch seine große Erfahrung sowie durch seine stete Unterstützung und Diskussionsbereitschaft konnte die Arbeit in der vorliegenden Form entstehen.

Herrn Prof. Dr.-Ing. M. Alber danke ich für sein Interesse an dieser Arbeit und die freundliche Bereitschaft zur Übernahme des Koreferats.

Prof. Dr.-Ing. M. Scherer danke ich für seine freundliche Bereitschaft als fachfremder Gutachter zu fungieren.

Allen Mitarbeitern der Arbeitsgruppe Experimentelle Mechanik danke ich für die stets gute Zusammenarbeit und die kollegiale Unterstützung. Herrn J. Banken und Herrn B. Thüner möchte ich besonders für Ihre Mitarbeit bei der Probenfertigung und Versuchsvorbereitung danken. Frau A. Kost gilt mein Dank für ihre Unterstützung und die Übernahme administrativer Aufgaben. Nicht zuletzt möchte ich mich bei Herrn Dr.-Ing. D. Bergmannshoff und Herrn Dr.-Ing. A. Bürgel für ihre Diskussionsbereitschaft und die Vorarbeiten zu dieser Arbeit bedanken.

Herrn Dr. D. R. Curran vom SRI International, Menlo Park, USA, danke ich dafür, daß er durch seine Mitarbeit als Gastwissenschaftler des Forschungsprojektes wertvolle Erkenntnisse beigesteuert hat. Herrn Dr. J. Grasa von der Universidad de Zaragoza, Spanien, danke ich für seine Hilfe bei der Erstellung des numerischen Modells. Herrn Prof. Dr. A. Fernández Canteli von der Universidad de Oviedo, Spanien, danke ich für lehrreiche Diskussionen zu verschieden Aspekten dieser Arbeit.

Bei Herrn Dipl.-Ing. L. Röchter und Herrn Dr. rer. nat. J. Nüchter bedanke ich mich für ihre große Hilfe bei der Durchsicht des fertigen Manuskripts.

Ebenfalls richtet sich mein Dank an die Mitarbeiter der Institute für Mechanik, Werkstoffe sowie für Geologie, Mineralogie und Geophysik, die durch viele nützliche Hilfestellungen und Diskussionen wesentlich zum Gelingen dieser Arbeit beigetragen haben.

Ganz herzlich möchte ich auch meiner Frau Agnieszka für ihre intensive Unterstützung, ihre Aufmunterung und ihre Geduld danken. 
Referenten: Prof. Dr.-Ing. J. F. Kalthoff Prof. Dr.-Ing. M. Alber Prof. Dr.-Ing. M. Scherer

Tag der Einreichung: 20.05 .2008 Tag der mündlichen Prüfung: 24.07.2008 


\section{Zusammenfassung}

Die Seismizität der oberen Erdkruste wird weitgehend von Bruchvorgängen kontrolliert. Das Verständnis der Sprödverformung von Krustengesteinen ist daher von großer gesellschaftlicher Relevanz. Die Kenntnis der Energiebilanz ist für ein umfassendes Verständnis der Mechanik von Erdbeben von entscheidender Bedeutung. Zum heutigen Zeitpunkt liegt eine geschlossene Beschreibung der energetischen Bedingungen der Bruchvorgänge im Verlauf von Erdbeben jedoch noch nicht vor. Verbesserte Erkenntnisse zu Erdbebenvorgängen bedingen eine genaue Charakterisierung der dissipativen Bruchausbreitungsprozesse. Ein Problem beim Aufstellen einer Energiebilanz ergibt sich daraus, daß es nicht möglich ist, Ergebnisse von Bruchexperimenten im Labormaßstab auf die Gegebenheiten in der Erdkruste zu übertragen, da sich bei herkömmlichen Laborexperimenten im Vergleich zu Erdbeben niedrigere Bruchenergien einstellen. Informationen zur Energiedissipation aus Laborexperimenten an Gesteinen liegen bislang lediglich für die Bruchinitiierung und für geringe Bruchausbreitungsgeschwindigkeiten vor. Es ist jedoch davon auszugehen, daß koseismische Bruchvorgänge sehr hohe Ausbreitungsgeschwindigkeiten erreichen, und weder eine rein statische Betrachtung der Rißinitiierung noch der -ausbreitung die aktiven Prozesse hinlänglich genau beschreiben. Anhand von Untersuchungen an anderen Materialien ist zudem bekannt, daß für dynamische Bruchvorgänge mit hohen Ausbreitungsgeschwindigkeiten, bei der Annäherung an eine maximale Bruchgeschwindigkeit, deutlich höhere Energiedissipationswerte resultieren als für langsame Bruchausbreitung bzw. -initiierung.

Im Rahmen der vorliegenden Arbeit werden Laborexperimente zur Bestimmung der Energiedissipation an laufenden Rissen in natürlichen Gesteinsproben durchgeführt. Dabei werden mehrere Größenordnungen der Rißausbreitungsrate abgedeckt. Ziel der Arbeit ist, einen Beitrag zur Verringerung der Differenz zwischen den bei natürlichen seismischen Ereignissen gemessenen Energiebilanzen und solchen aus Laborexperimenten zu leisten. Bruchenergien und Bruchgeschwindigkeiten werden mit einem optimierten Dehnungsmeßstreifen-Verfahren bestimmt. Methoden der Schlag/Stoßbelastung werden eingesetzt, um durch die Erzeugung eines Energieüberangebotes bei der Initiierung Risse in Laborproben endlicher Abmessungen auf hohe Ausbreitungsgeschwindigkeiten zu beschleunigen. Zusätzlich werden die Bedingungen bei der Initiierung der Rißlaufereignisse untersucht.

Die Messungen werden an den Gesteinen Solnhofener Kalkstein und Arkansas Novaculit durchgeführt. Mit der Methode der Stoßbelastung werden die maximalen Bruchgeschwindigkeiten der Gesteine erreicht. Die ermittelten Bruchenergien weisen im Bereich der maximalen Bruchgeschwindigkeit einen starken Anstieg auf. Zusätzlich wird ein zweiter starker Anstieg der Bruchenergien, der sich für die stabile subkritische Rißerweiterung im Bereich langsamer Rißausbreitungsgeschwindigkeiten ergibt, bis zum Übergang zur überkritischen Rißerweiterung quantitativ erfasst. Zwischen diesen Bereichen stark ansteigender Bruchenergien ist die Variation der 
Bruchenergie mit der Bruchgeschwindigkeit gering. Insgesamt ergeben sich für beide Gesteine unter dynamischen Bedingungen bei hohen Bruchausbreitungsgeschwindigkeiten Bruchenergien, die signifikant größer sind als für langsame Rißausbreitung bzw. als für Rißinitiierung. Durch die Bestimmung der Bruchenergie bei hohen Ausbreitungsgeschwindigkeiten konnten durch die durchgeführten Experimente neuartige Erkenntnisse für die Energiedissipation bei dynamischen Bruchvorgängen in Gesteinen gewonnen werden. Die Ergebnisse können daher dazu verwendet werden, verbesserte Energiebilanzmodelle bei Erdbeben aufzustellen. 


\section{Inhaltsverzeichnis}

Symbol- und Abkürzungsverzeichnis iv

1 Einleitung $\quad 1$

2 Grundlagen $\quad 3$

2.1 Linear-elastische Bruchmechanik ................ 3

2.1.1 Spannungskonzentrationen - das Irwinsche $K$-Konzept . . . . . 3

2.1.2 Bruchzähigkeit . . . . . . . . . . . . . 8

2.1.3 Prozeßzone .................... 8

2.1.4 Gültigkeitsgrenzen der linear-elastischen Bruchmechanik . . . . 11

2.1.5 Energieansatz - das Griffithsche G-Konzept . . . . . . . . . 13

2.2 Bruchausbreitung . . . . . . . . . . . . . . 16

2.2.1 Bruchausbreitungskriterium . . . . . . . . . . . 16

2.2.2 Bruchausbreitungsrichtung . . . . . . . . . . . . 19

2.2.3 Rißinitiierung bei gemischter Beanspruchung . . . . . . . . 21

2.3 Dynamische Erweiterung der linear-elastischen Bruchmechanik . . . . 22

2.3.1 Vorbemerkungen ................. 22

2.3.2 Elastische Wellen . . . . . . . . . . . . . . . . 22

2.3.3 Dynamische Effekte bei stationären Rissen . . . . . . . . . 23

2.3.4 Dynamische Effekte bei instabiler Rißverlängerung . . . . . . . 25

2.4 Anwendung der Bruchmechanik auf geologische Materialien . . . . . . 32

3 Methodik $\quad 36$

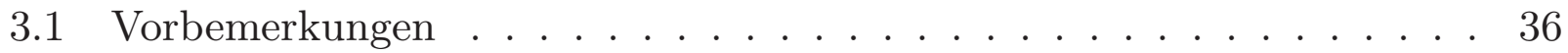

3.2 Bestimmung von Rißlaufbruchzähigkeiten mit dem optimierten Dehnungsmeßstreifen-Ketten-Verfahren . . . . . . . . . . . . . . . 36

3.2.1 Prinzip des optimierten Dehnungsmeßstreifenverfahrens bei stationären Rissen . . . . . . . . . . . . . . . 36 
3.2.2 Dehnungsmeßstreifen-Ketten-Verfahren bei laufenden Rissen

3.3 Methoden zur Erzeugung von Rißlaufereignissen unter Laborbedingungen von der Initiierung bis in den Bereich der maximalen Geschwindigkeit ..................... . . . 46

3.3.1 Einflüsse der Probengeometrie und der Belastungsart auf die Erzeugung von Rißlaufereignissen bei quasistatischer Beanspruchung . . . . . . . . . . . . . . . 46

3.3.2 Erzeugung schneller Rißausbreitung durch dynamische Beanspruchung ................. . . . 49

3.3.3 Einsatz von Scher(Modus-II)-Beanspruchungsbedingungen zur Erzeugung von Rißlaufereignissen mit hoher Bruchgeschwindigkeit ................... . . 50

3.3.4 Einsatz dynamischer Scher(Modus-II)-Beanspruchungsbedingungen mit der LECEI-Technik zur Erzeugung von Rißlaufereignissen mit maximaler Bruchgeschwindigkeit . . . . . . . . 51

3.4 Globalenergie-Verfahren . . . . . . . . . . . . . . . . 55

3.5 Bruchgeschwindigkeitsabschätzung mit Wallnerlinien . . . . . . . . 56

4 Ergebnisse

4.1 Probenmaterial und Probendimensionierung . . . . . . . . . . 59

4.2 Erzeugung von Rißlaufereignissen in Gestein . . . . . . . . . . . . . . 61

4.3 Experimentell in Gestein erzielte Bruchgeschwindigkeiten . . . . . . . 63

4.4 Meßdatengewinnung . . . . . . . . . . . . . . . . . 66

4.4.1 Vorbemerkungen ................ 66

4.4.2 Dehnungsmeßstreifensignale zur Rißlaufzähigkeitsmessung an Gestein ... . . . . . . . . . . . . 67

4.5 Gesamtergebnisse . . . . . . . . . . . . . . 73

4.6 Vergleich der Ergebnisse durch mikroskopische Untersuchungen . . . . 77

4.7 Kontroll- und Vergleichsmessungen . . . . . . . . . . . . . . . 81

5 Diskussion $\quad 86$

$\begin{array}{ll}\text { Literaturverzeichnis } & 91\end{array}$ 
Anhang I I

A Ergänzende Messungen an Gestein . . . . . . . . . . . . . . . I

B Zur Meßgenauigkeit/Mehrdeutigkeit von DMS-Signalen bei laufenden Rissen . . . . . . . . . . . . . . . . . . VI

C Bestimmung von Spannungsintensitätsfaktoren . . . . . . . . . XI

D Ablauf des LECEI-Experimentes . . . . . . . . . . . . XIII

E Finite-Elemente-Modellierung des LECEI-Experimentes . . . . . . . . XV F Ergebnistabellen .................. XIX 


\section{Symbol- und Abkürzungsverzeichnis}

\section{Lateinische Buchstaben}

\begin{tabular}{|c|c|}
\hline$a$ & Rißlänge \\
\hline$A_{0}, A_{1}, B_{0}, B_{1}$ & Koeffizienten höherer Terme der Rißspitzenspannungsverteilung \\
\hline$c$ & Schallgeschwindigkeit \\
\hline$C O D$ & Rißöffnung \\
\hline$B$ & Probendicke \\
\hline DMS & Dehnungsmeßstreifen \\
\hline$E$ & Elastizitätsmodul \\
\hline$E_{\text {kin }}$ & kinetische Energie \\
\hline ESZ & Ebener Spannungszustand \\
\hline EDZ & Ebener Dehnungszustand \\
\hline$f$ & Funktionen der Spannungsverteilung an einem Riß \\
\hline$F$ & Kraft, äußere Belastung \\
\hline $\begin{array}{l}G_{I}, G_{I I}, G_{I I I} \\
G_{C}\end{array}$ & $\begin{array}{l}\text { Energiefreisetzungsrate, Modus-I, -II und -III } \\
\text { spezifische Bruchenergie }\end{array}$ \\
\hline$G_{c}^{*}$ & spezifische Initiierungsenergie bei Kerben \\
\hline$G_{d}^{*}$ & spezifische dynamische Initiierungsenergie bei Kerben \\
\hline$G_{I D}$ & spezifische Bruchausbreitungsenergie, Modus-I \\
\hline$K_{I}, K_{I I}, K_{I I I}$ & Spannungsintensitätsfaktoren, Modus-I, -II und -III \\
\hline$\dot{K}_{I}, \dot{K}_{I I}$ & Belastungsrate, Modus-I und -II \\
\hline$K_{c}$ & Bruchzähigkeit \\
\hline$K_{c}^{*}$ & Bruchzähigkeit bei Kerben \\
\hline$K_{c}^{i}$ bzw. $G_{c}^{i}$ & Beginn instabiler Rißverlängerung \\
\hline$K_{c}^{s}$ bzw. $G_{c}^{s}$ & Beginn stabiler Rißverlängerung \\
\hline$K_{I d}, K_{I I d}$ & dynamische Initiierungszähigkeit, Modus-I, und -II \\
\hline$K_{d}^{*}$ & dynamische Initiierungszähigkeit bei Kerben \\
\hline$K_{I D}$ & Rißlaufzähigkeit, Modus-I \\
\hline$L$ & Probenbreite \\
\hline$r_{p}$ & Prozeßzonenradius \\
\hline$R$ & Rißwiderstand \\
\hline$s$ & Auflagerabstand \\
\hline SIF & Spannungsintensitätsfaktor \\
\hline$t$ & Zeit \\
\hline$U_{d}$ & dissipierte Energie \\
\hline$U_{p}$ & elastische Dehnungsenergie \\
\hline$U_{p}^{s}$ & Rißschließenergie \\
\hline$v_{0}$ & Belastungs-, Schlag-/Stoßge \\
\hline
\end{tabular}




$\begin{array}{ll}v_{c} & \text { Rißlaufgeschwindigkeit } \\ v_{p} & \text { Longitudinal-/Druckwellengeschwindigkeit } \\ v_{s} & \text { Transversal-/Scherwellengeschwindigkeit } \\ v_{r} & \text { Rißuferverschiebung } \\ v_{R} & \text { Rayleigh-/Oberflächenwellengeschwindigkeit } \\ V & \text { Volumen } \\ W & \text { Probenhöhe } \\ W^{a} & \text { äußere Arbeit }\end{array}$

\section{Griechische Buchstaben}

$\alpha$

$\epsilon$

$\epsilon_{\alpha}$

$\epsilon_{\mathrm{DMS}}$

$\gamma$

$\mu$

$\nu$

$\phi$

$\rho$

$\varrho$

$\sigma$

$\sigma_{f}$

$\sigma_{Y S}$

$\sigma_{t}$

$\tau$
Meßrichtung eines Dehnungsmeßstreifens

Dehnung

gerichtete Dehnung, Meßrichtung $\alpha$

Durch Dehnungsmeßstreifen gemessene Dehnung

Scherdehnung

Schermodul

Poissonzahl

Porosität

Kerbradius

Dichte

Spannung

Materialfestigkeit

Fließspannung

Zugfestigkeit

Scherspannung

\section{Indizes und Koordinatenbezeichnungen}

$$
\begin{aligned}
& i, j \\
& x, y, z \\
& r, \varphi, z \\
& I, I I, I I I
\end{aligned}
$$

Komponentennotation

karthesische Koordinaten

Polar- und Zylinderkoordinaten

Beanspruchungsmoden 



\section{$1 \quad$ Einleitung}

Die Ausbreitung von Rissen in Gestein ist ein alltägliches Naturphänomen mit direkt spürbaren Auswirkungen. Als Erdbeben initiieren Risse in der Erde vor allem in der spröden Oberkruste (Schizosphäre) an präexistierenden Schwächezonen infolge tektonischer Beanspruchungen. Bruchvorgänge sind aber auch für Sicherheitsabwägungen im Rahmen geotechnischer Fragestellungen von Bedeutung. Sie sind ein entscheidender Faktor bei Stabilitätsvorhersagen mit gesellschaftlicher und wirtschaftlicher Relevanz und betreffen weite Gebiete, über die Gefährdung durch Erdbeben und Tsunamis oder anthropogen induzierte Versagensfälle bis hin zur Nutzung unterirdischer Energieressourcen.

Für eine umfassende Betrachtung von Bruchprozessen ist vor allem die Kenntnis der Energiedissipation in den verschiedenen Phasen eines Rißlaufereignisses, für die sich bei der Initiierung und im Verlauf der Ausbreitung unterschiedliche Energieniveaus einstellen, von Bedeutung. Dabei sind die Vorgänge bei Bruchprozessen in der Erde hochkompliziert und unterliegen vielfältigen Einflüssen u. a. im Beanspruchungszustand und Materialaufbau der betroffenen Strukturen. Untersuchungen zum Verständnis des Bruchverhaltens von Gestein werden einerseits direkt, an Proben im Labor, und andererseits indirekt, in situ im Inneren der Erde beispielsweise durch Inversion seismischer Wellenfelder, durchgeführt. Ergebnisse hieraus können bisher aufgrund der großen Abweichungen der betreffenden Längen- und Zeitskalen nicht zur Übereinstimmung gebracht werden. Labor- und Feldbeobachtungen berücksichtigen mit den zur Zeit eingesetzten Methoden zudem nicht die Abhängigkeit der Bruchvorgänge von der Ausbreitungsgeschwindigkeit, sondern bestimmen Versagensparameter lediglich mittelnd über den tatsächlichen Geschwindigkeitsverlauf. Somit erfassen sie nicht die Variation der Energiedissipation mit der Bruchgeschwindigkeit. Zur Erfassung solcher geschwindigkeitsabhängiger Effekte wird im Rahmen dieser Arbeit der Bruchvorgang auf den einfachsten Fall, den der Zugrißausbreitung in homogenen Gesteinen, zurückgeführt und mittels der Methoden der linear-elastischen Bruchmechanik in Laborexperimenten quantifiziert. Ziel der Arbeit ist es, durch die Messung der Energiebilanz an laufenden Rissen in Gesteinsproben, von der Initiierung bis in den Bereich hoher Ausbreitungsgeschwindigkeiten, zum besseren Verständnis von Bruchvorgängen in der Erde beizutragen.

Die Energiedissipation wird auf der Grundlage der Analogie zwischen der spezifischen Bruchenergie und der Bruchzähigkeit unter Berücksichtigung dynamischer Effekte in Form von kritischen Spannungsintensitätsfaktoren als Rißlaufzähigkeiten $K_{I D}$ in Abhängigkeit der Ausbreitungsgeschwindigkeit des Risses $v_{c}$ ermittelt; dabei entspricht die spezifische Bruchenergie dem Quadrat der Bruchzähigkeit: $G_{I D}\left(v_{c}\right) \sim$ $K_{I D}^{2}\left(v_{c}\right)$. Rißlaufzähigkeiten werden mit einem optimierten Dehnungsmeßstreifen- 
Ketten-Verfahren entlang des Rißpfades, unter Berücksichtigung höherer Glieder, aus dem Rißspitzenfeld mit hochauflösenden Methoden gemessen. Mit diesem Verfahren sind sowohl die Höhe der Rißlaufzähigkeit als auch die der momentanen Ausbreitungsgeschwindigkeit bis in den Bereich sehr hoher Rißlaufgeschwindigkeiten simultan bestimmbar. Ergänzend werden die Bedingungen bei der Rißinitiierung sowohl für quasistatische $\left(K_{I c}\right)$ als auch für dynamische $\left(K_{I d}, K_{I I d}\right)$ Beanspruchungsbedingungen erfasst. Neben der Verwendung herkömmlicher quasistatischer Belastung zur Initiierung langsamer Rißlaufereignisse werden dynamische Belastungstechniken unter Zug(Modus-I)- und unter Scher(Modus-II)-Bedingungen dazu eingesetzt, ein Energieüberangebot bei der Rißinitiierung zu erzeugen. Dadurch werden Risse in Laborproben mit notwendigerweise beschränkten Abmessung auf maximale Rißausbreitungsgeschwindigkeiten beschleunigt. Somit werden Ergebnisse zur Messung der Energiedissipation für den gesamten möglichen Rißgeschwindigkeitsbereich erzielt. Das Schadensbild entlang des Rißpfades wird mit mikroskopischen Methoden untersucht. Die Ergebnisse werden durch Kontroll- und Vergleichsexperimente verifiziert.

Auf diese Einleitung folgt in Kapitel 2 eine Einführung in die Grundlagen der linearelastischen Bruchmechanik. Im Anschluß werden die Bedingungen bei der Ausbreitung von Rissen vorgestellt und ein Überblick über dynamische Einflüsse auf Bruchvorgänge, besonders im Hinblick auf hohe Rißausbreitungsgeschwindigkeiten angegeben. Das Kapitel wird mit einer Literaturstudie zum Stand der Forschung bei der Anwendung der Bruchmechanik auf Gesteine abgeschlossen.

In Kapitel 3 werden die eingesetzten Methoden erläutert. Zuerst wird die Technik der Bestimmung der momentanen Werte von Rißlaufzähigkeit und Rißlaufgeschwindigkeit im Verlauf eines Rißlaufereignisses vorgestellt. Dann werden die Methoden zur Erzeugung von Rißlaufereignissen in Gestein, speziell bis in den Bereich hoher Ausbreitungsgeschwindigkeiten unter Schlag-/Stoßbelastungsbedingungen beschrieben.

In Kapitel 4 werden die erzielten Ergebnisse präsentiert. Zunächst werden die untersuchten Gesteine charakterisiert. Dann wird auf die Besonderheiten des Bruchverhaltens von Gesteinen im Vergleich zu herkömmlichen sprödbrechenden Materialien und auf den Einfluß der eingesetzten Belastungsmethoden auf die erzielten Rißlaufgeschwindigkeiten eingegangen. Schließlich werden die mit dem Dehnungsmeßstreifen-Ketten-Verfahren ermittelten Rißlaufzähigkeitsdaten im Zusammenhang mit dem experimentellen Rahmen erläutert und im Gesamtüberblick über dem Geschwindigkeitsverlauf angegeben. Mit Bezug auf die erzielten Ergebnisse werden im Anschluß die mikroskopischen Untersuchungen vorgestellt. Den letzten Abschnitt des Kapitels bildet die Bestätigung bestimmter Aspekte der Ergebnisse durch Kontrollund Vergleichsmessungen.

Abschließend wird in Kapitel 5 die im Spannungs- $K$-Bild ermittelte BruchzähigkeitsBruchgeschwindigkeits-Beziehung diskutiert und die Übertragung der Ergebnisse in das Energie-G-Bild angegeben. Dabei wird auf die Bedeutung der Ergebnisse für Bruchvorgänge in der Erde verwiesen. 


\section{Grundlagen}

\subsection{Linear-elastische Bruchmechanik}

\subsubsection{Spannungskonzentrationen - das Irwinsche $K$-Konzept}

Das Versagensverhalten eines belasteten Festkörpers wird entscheidend durch das Vorhandensein von Rissen bestimmt. Ausgehend von dieser Beobachtung wurde vor etwa hundert Jahren erkannt, daß die an Rißspitzen auftretenden Spannungsüberhöhungen oder Spannungskonzentrationen ursächlich für die Rißausbreitung und somit letztlich für den Versagensprozeß sind. Daraufhin wurden Wege gesucht, dies im Rahmen einer mechanischen Betrachtung zu quantifizieren, um somit verlässliche Sicherheitsaussagen zu ermöglichen. Den entscheidenden Durchbruch erzielte G. R. Irwin in der Mitte des 20. Jahrhunderts durch die Einführung des bruchmechanischen $K$-Konzeptes, das mit dem Spannungsintensitätsfaktor $K$ als Rißspitzenparameter ein Maß für die Gefährlichkeit und mit der hierüber definierten Bruchzähigkeit ein Maß für den Widerstand eines Materials gegen Bruchausbreitung liefert [1-8]. Das $K$-Konzept beschreibt im wesentlichen sprödes Bruchverhalten, bei dem Abweichungen von linear-elastischem Materialverhalten auf eine schmale Zone in der Nähe der Rißufer beschränkt sind.

Zur Beschreibung der Belastungssituation eines rißbehafteten Körpers werden in der Bruchmechanik drei fundamentale Beanspruchungsmoden eingeführt (Abb. 1), aus denen sich durch Superposition sämtliche Belastungsfälle zusammensetzen lassen. Man unterscheidet, relativ zur Lage des Risses, zwischen:

- Modus-I - Zugbeanspruchung,

- Modus-II - ebener Scherung und

- Modus-III - nichtebener Scherung.
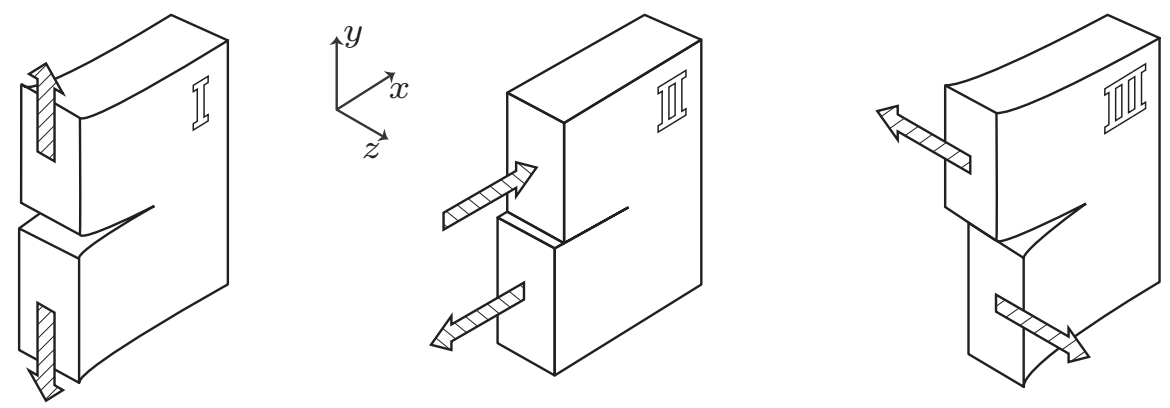

Abb. 1: Fundamentale Beanspruchungsmoden eines Risses. 
Bezüglich der Belastungssituation läßst sich, als Randwertproblem formuliert, mithilfe der linearen Elastizitätstheorie jeweils das die Beanspruchung bestimmende Spannungsfeld an der Rißspitze ableiten [9-13]. Die Charakteristika solcher Rißspitzenfelder verdeutlicht ein idealisiertes Beispiel.

Betrachtet wird eine unendlich ausgedehnten Scheibe mit einem scharfen Innenriß der Länge $2 a$ unter einer äußeren Zugbelastung $\sigma_{0}$ in $y$-Richtung, d.h. einer reinen Modus-I-Beanspruchung (Abb.2). Obwohl, wie im vorliegenden Beispiel der Fall, unter Umständen nur eine einachsige äußere Belastung vorliegt, resultiert in Rißspitzennähe ein mehrachsiger Beanspruchungszustand. Wie viele andere Rißprobleme läßt sich dieses Beispiel auf ebene elastische Zustände reduzieren. Hierbei verschwindet für den ebenen Spannungszustand (ESZ) und den ebenen Dehnungszustand (EDZ) jeweils eine der Hauptspannungen bzw. -dehnungen. Für den ebenen Spannungszustand gilt: $\sigma_{z}=0$ und für den ebenen Dehnungszustand: $\epsilon_{z}=0$ sowie $\sigma_{z}=\nu\left(\sigma_{x}+\sigma_{y}\right)$. Des weiteren verschwinden für beide Zustände die folgenden gemischten deviatorischen Komponenten: $\gamma_{x z}=\gamma_{y z}=\tau_{x z}=\tau_{y z}=0$. Dabei gibt der ebene Spannungszustand die Gegebenheiten an der Scheibenoberfläche wieder und der ebene Dehnungszustand gilt für das Scheibeninnere, mit den entsprechenden Auswirkungen für die Ausbildung der plastischen Zone (siehe 2.1.3). Nach einer Lösung von Sneddon [10] lautet das Spannungsnahfeld $(r \rightarrow 0)$ für die rechte Rißspitze:

$$
\begin{aligned}
\sigma_{x} & =\frac{\sigma_{0} \sqrt{\pi a}}{\sqrt{2 \pi r}} \cos \frac{\varphi}{2}\left(1-\sin \frac{\varphi}{2} \sin \frac{3 \varphi}{2}\right), \\
\sigma_{y} & =\frac{\sigma_{0} \sqrt{\pi a}}{\sqrt{2 \pi r}} \cos \frac{\varphi}{2}\left(1+\sin \frac{\varphi}{2} \sin \frac{3 \varphi}{2}\right), \\
\tau_{x y} & =\frac{\sigma_{0} \sqrt{\pi a}}{\sqrt{2 \pi r}} \sin \frac{\varphi}{2} \cos \frac{\varphi}{2} \cos \frac{3 \varphi}{2}
\end{aligned}
$$

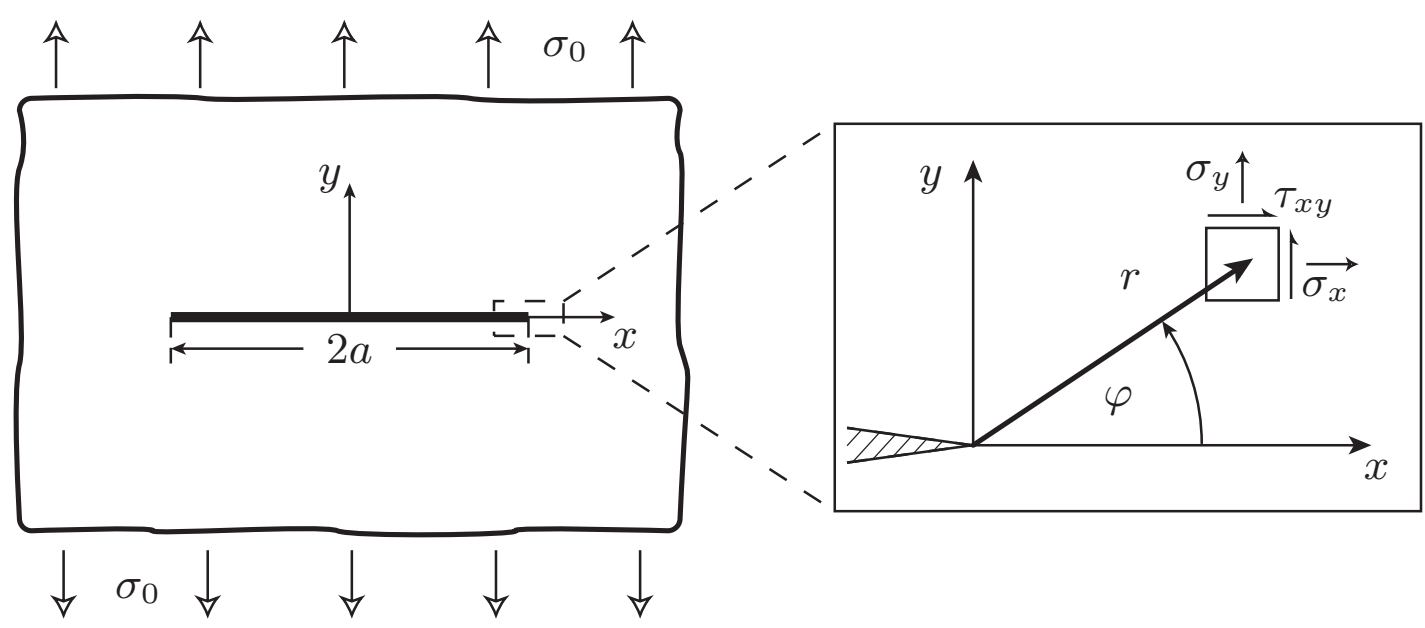

Abb. 2: Unendlich ausgedehnte Scheibe mit Innenriß unter gleichförmigem Zug. 
und die Verschiebungen $u$ (in $x$-Richtung) und $v$ (in $y$-Richtung) in Rißspitzennähe lassen sich angeben als (z. B. [14]):

$$
\begin{aligned}
& u=\frac{\sigma_{0} \sqrt{\pi a}}{\mu} \sqrt{\frac{r}{2 \pi}} \cos \frac{\varphi}{2}\left(\kappa-1+2 \sin ^{2} \frac{\varphi}{2}\right), \\
& v=\frac{\sigma_{0} \sqrt{\pi a}}{\mu} \sqrt{\frac{r}{2 \pi}} \sin \frac{\varphi}{2}\left(\kappa+1-2 \cos ^{2} \frac{\varphi}{2}\right)
\end{aligned}
$$

mit: $\quad \mu=\frac{E}{2(1+\nu)}$

$$
\begin{aligned}
\text { und: } & \kappa=\frac{3-\nu}{1+\nu} & & (E S Z) \\
\kappa & =3-4 \nu & & (E D Z) .
\end{aligned}
$$

Das Spannungsfeld Gl. (1) weist eine Singularität mit $1 / \sqrt{r}$ auf (Abb.3), deren Stärke durch die äußere Spannung $\sigma_{0}$ und die Rißlänge $a$ bestimmt wird. Dieses Verhalten ergibt sich analog für die anderen Beanspruchungsmoden. Die Spannungssingularität ist charakteristisch für sämtliche elastischen Rißprobleme, so daß das Rißspitzenspannungsfeld immer gegeben ist durch:

$$
\sigma_{i j}=\frac{K_{I, I I, I I I}}{\sqrt{2 \pi r}} f_{i j}^{I, I I, I I I}(\varphi)
$$

wobei die durch $f_{i j}(\varphi)$ beschriebene Winkelabhängigkeit für den jeweiligen Modus eine eigene Form annimmt und mit dem Spannungsintensitätsfaktor $K$ sämtliche

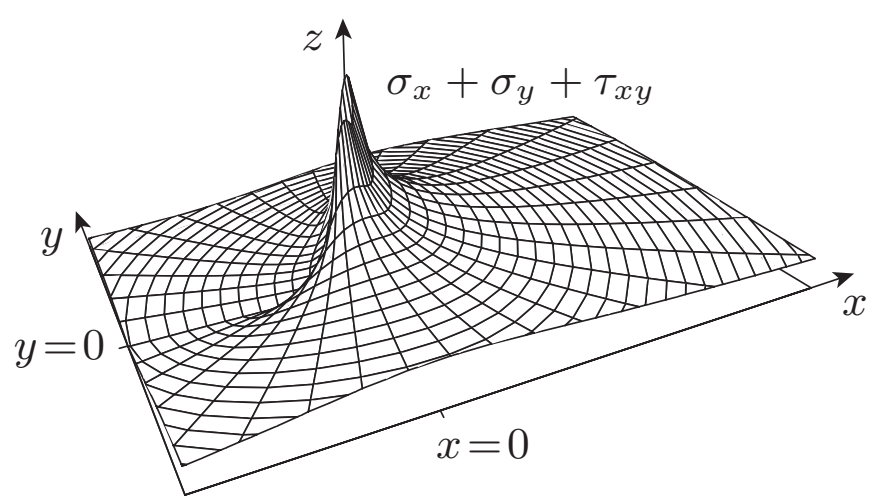

a)

Abb. 3: Rißspitzenspannungsfeld eines scharfen Risses unter Modus-I-Beanspruchung nach der Sneddon-Lösung: a) Spannungssumme in der Ebene und b) für das Ligament $(y=0, \varphi=0, x>0)$.

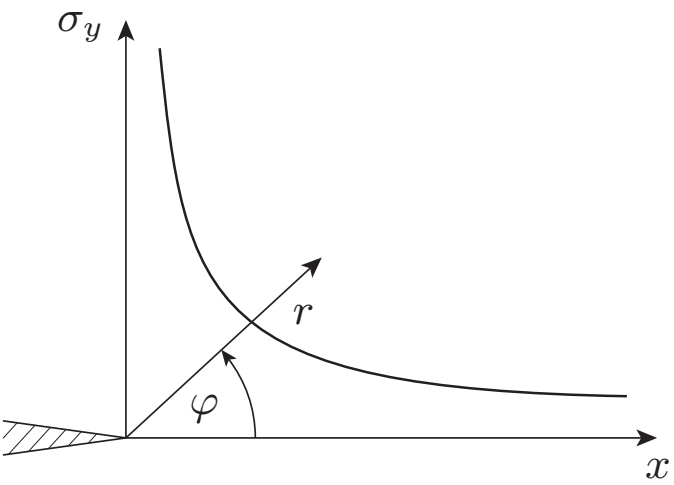

b) 
Parameter, die nicht von $r$ und $\varphi$ abhängen, zusammengefaßst werden. Der Spannungsintensitätsfaktor ist auf dem Ligament $(y=\varphi=0)$ für den Grenzübergang $r \rightarrow 0$ definiert als:

$$
\begin{aligned}
K_{I} & :=\lim _{r \rightarrow 0} \sigma_{y}(r, \varphi=0) \sqrt{2 \pi r}, \\
K_{I I} & :=\lim _{r \rightarrow 0} \tau_{x y}(r, \varphi=0) \sqrt{2 \pi r}, \\
K_{I I I} & :=\lim _{r \rightarrow 0} \tau_{z y}(r, \varphi=0) \sqrt{2 \pi r} .
\end{aligned}
$$

Die hier angegebene Definition bezieht sich, formal gesehen, ausschließlich auf scharfe Risse. Spannungsintensitätsfaktoren sind ein Maß für die Stärke der Spannungsumlagerung durch das Vorhandensein des Risses. An Rissen mit abgerundeten Rißspitzen - im folgenden zur Unterscheidung von scharfen Rissen als „Kerben“ bezeichnet - vollzieht sich diese Spannungsumlagerung in der Nähe der Rißspitze in abgeschwächtem Maße, die sich ausbildenden Spannungskonzentrationen weisen eine Proportionalität zum Grad der Kerbschärfe auf (zur Übertragbarkeit des $K$-Konzeptes auf abgerundete Kerben siehe [15]; siehe auch Abschnitt 3.2, [16, 17]).

Die Sneddon-Lösung, die für den Nahbereich $(r \rightarrow 0)$ eine gute Näherung für das Spannungsfeld liefert, kann für größere Entfernungen von der Rißspitze nicht verwendet werden. Eine allgemeine Lösung, die für beliebige Entfernungen das Spannungsfeld exakt beschreibt, wird nach Williams [12,18] durch folgenden Reihenansatz repräsentiert:

$$
\sigma_{i j}=\sum_{n=0}^{\infty} C_{n} r^{\frac{n-1}{2}} f_{n, i j}(\varphi)
$$

Dabei liefert das erste Glied der Reihe wiederum das - mit der Sneddon-Lösung identische - Nahfeld. Daher lautet Gl. (5a), mit $C_{0}=K / \sqrt{2 \pi}$ bzw. mit $A_{0}=K / \sqrt{2 \pi}$, benutzt man den Buchstaben $A$, um die Faktoren von halbzahligen und den Buchstaben $B$, um die Faktoren der ganzzahligen Exponenten von $r$ zu kennzeichnen, in ausgeschriebener Form:

$$
\sigma_{i j}=\frac{K}{\sqrt{2 \pi r}} f_{0, i j}(\varphi)+B_{0} r^{0} f_{1, i j}(\varphi)+A_{1} r^{\frac{1}{2}} f_{2, i j}(\varphi)+\cdots
$$

Es wird deutlich, daß das Spannungsfeld für kleine Entfernungen von der Rißspitze durch die Singularität des durch den Spannungsintensitätsfaktor bestimmten Reihengliedes dominiert wird. Die Glieder höherer Ordnung in $r$ (höhere Glieder) stellen für die Bestimmung des Spannungsintensitätsfaktors $K$ Störterme dar. Diese Störterme gewinnen erst für wachsende Abstände $r$ von der Rißspitze immer stärker an Bedeutung. Davon ausgenommen ist der zweite, der $B_{0}$-Term, der nicht vom Abstand $r$ von der Rißspitze abhängt - daher auch bezeichnet als „,konstanter Term“* Dieser

\footnotetext{
* Ebenfalls, gerade in der englischsprachigen Literatur, ist die Bezeichnung ,T-Stress" gebräuchlich [19].
} 
Term wird häufig bei der Bestimmung einer Näherungslösung für das Rißspitzenspannungsnahfeld berücksichtigt [11,20], er liefert grundsätzlich, d. h. für jeden Beanspruchungsmodus, nur Beiträge zur $x$-Normalkomponente $\left(\sigma_{x}\right)$ des Spannungsfeldes. Die Beiträge der höheren Glieder der Spannungsverteilung zum gesamten Spannungsfeld sind nur durch aufwendige Analysen [21] bzw. experimentell, z. B. mittels des spannungsoptischen Meßverfahrens [12,22, 23], zu bestimmen.

Die die Stärke des Spannungsfeldes bestimmenden Faktoren $C_{n}$, also insbesondere auch der Spannungsintensitätsfaktor $K$, enthalten, neben der Information über die Belastung und die Rißgeometrie, die der jeweiligen Körpergeometrie angepaßten Randbedingungen. Für den vorliegenden Spezialfall der unendlich ausgedehnten Scheibe und über die eingangs getroffene Beschränkung auf den Modus-I-Fall hinaus, auch bei Vorliegen einer jeweils reinen Modus-II- oder Modus-III-Beanspruchung durch die äußere Belastung $\tau_{0, x y}$ bzw. $\tau_{0, x z}$, ergibt sich für die Spannungsintensitätsfaktoren des Beispiels:

$$
K_{I}=\sigma_{0, y} \sqrt{\pi a}, \quad K_{I I}=\tau_{0, x y} \sqrt{\pi a} \quad \text { und } \quad K_{I I I}=\tau_{0, x z} \sqrt{\pi a} .
$$

Für den Beispielsfall sind durch die einfache Körpergeometrie nur die Belastung sowie die Rißlänge selbst und sonst keine weiteren Randbedingungen zu beachten. Die Abhängigkeit der Spannungsintensitätsfaktoren von Geometrie- und Beanspruchungsparametern sind für eine große Anzahl von Beispielen in Form von Katalogen oder Handbüchern zusammengefaßt [24-26]. Bei überlagerten Belastungen des gleichen Modus sind, bei Vorliegen einer identischen Verteilung des Spannungsfeldes in Rißspitzennähe (gleiche $r$-, $\varphi$-Abhängigkeit), nach dem Superpositionsprinzip die resultierenden Spannungsintensitätsfaktoren zu addieren: $K_{i}^{\text {ges }}=\sum_{n=1}^{N} K_{i, n}(i=I, I I, I I I)$. Abbildung 4 zeigt dazu im Modus-I das Beispiel einer durch gleichmäßigen Zug beanspruchten Probe mit einem Randriß. Der an dem Probenrand angreifenden Spannung ist eine Belastung durch ein Kräftepaar in Rißnähe überlagert. Eine Kombination von Beanspruchungen unterschiedlicher Moden ist dagegen nicht zulässig.
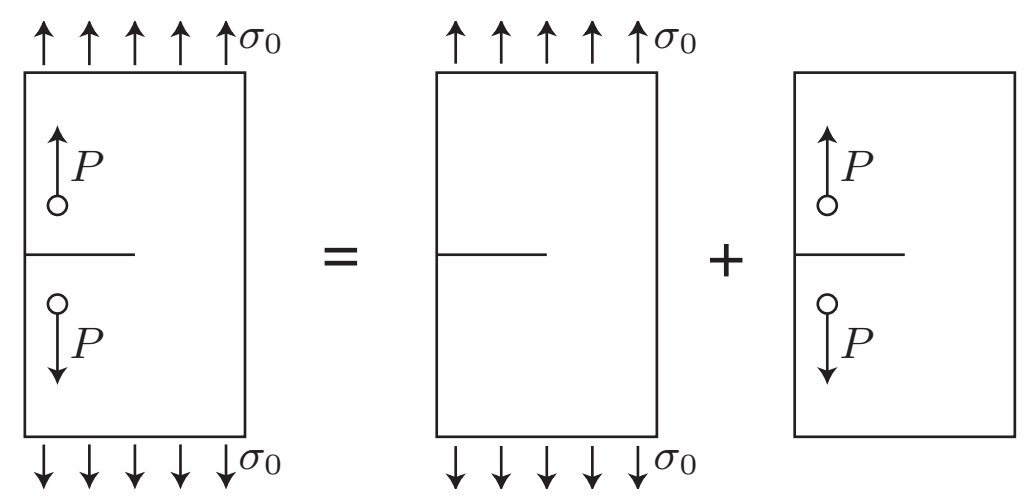

Abb. 4: Superposition von Spannungsintensitätsfaktoren bei überlagerter Beanspruchung des gleichen Modus (hier: Angreifen einer äußeren Spannung $\sigma_{0}$ und eines Kräftepaares $P$ im Modus-I). 


\subsubsection{Bruchzähigkeit}

Mithilfe der Spannungsintensitätsfaktoren läßt sich nun ein Bruchkriterium formulieren: Der kritische Wert des Spannungsintensitätsfaktors, bei dem Rißinstabilität ohne weitere Erhöhung der Belastung einsetzt, wird als Bruchzähigkeit $K_{c}$ bezeichnet [27].

Die Bruchzähigkeit ist ein Maß für den Widerstand eines Materials gegen Rißausbreitung. Sie stellt - im Gegensatz zu dem Spannungsintensitätsfaktor, der als Maß für die Höhe der lokalen Beanspruchung eine rein mechanische Größe angibt - einen charakteristischen Materialkennwert dar. Dieser Kennwert ist konstant, wenn Oberflächeneffekte vernachlässigbar sind. Bei der experimentellen Ermittlung konstanter Bruchzähigkeiten $K_{I c}$ müssen dazu bestimmte Gültigkeitsbedingungen eingehalten werden, auf die in Abschnitt 2.1.4 eingegangen wird. Als Materialkennwert wird die Bruchzähigkeit von den Eigenschaften des Materials bestimmt, dazu zählen u. a. die Mikrostruktur sowie eventuelle Wärmebehandlungen. Zusätzlich ist diese Größe von anderen Einflußfaktoren, wie der Belastungshistorie, der Temperatur oder dem Umgebungsmedium abhängig.

\subsubsection{Prozeßzone}

In den vorstehend angeführten Betrachtungen wird linear-elastisches Materialverhalten für den gesamten Körper und den gesamten Belastungsvorgang angenommen. Unter diesen idealisierten Bedingungen treten in Rißspitzennähe theoretisch unendlich hohe Spannungen auf. Jedes reale Material reagiert jedoch nur bis zu einem endlichen Spannungsniveau linear-elastisch; steigt die Beanspruchung über dieses durch das jeweilige Materialverhalten festgelegte Niveau, setzt irreversible Materialschädigung ein. Durch das Ausbilden von Spannungskonzentrationen ist diese Materialschädigung bei Rißproblemen auf eine Zone in der Nähe der Rißspitze beschränkt, deren Größe - bis zum Erreichen des kritischen Wertes - von der Stärke der Beanspruchung abhängt.

Nach einem Modell von Irwin [27] erstreckt sich diese inelastische Zone über ein kreisrundes Gebiet, dessen Radius $r_{p}$ sich für eine Modus-I-Beanspruchung aus dem elastischen Spannungsfeld (Gl. 1, $\varphi=0$ ) ergibt zu:

$$
r_{p}=\frac{1}{2 \pi}\left(\frac{K_{I}}{\sigma_{f}}\right)^{2}
$$

Hierbei ist $\sigma_{f}$ die Grenzspannung, die, hier im Rahmen eines Normalspannungskriteriums, von den anliegenden Spannungen zu überschreiten ist, um Materialschädigung zu erzeugen. Für diesen Festigkeitswert ist eine dem Materialverhalten entsprechende Größe anzusetzen; bei metallischen Materialien ist dies die Fließgrenze $\sigma_{Y S}$, bei Gesteinen wird jedoch die Zugfestigkeit $\sigma_{t}$ verwendet (siehe auch Abschnitt 2.4, [28-30]). 
Für die Probenoberfläche und das Probeninnere ergeben sich aufgrund der unterschiedlichen Mehrachsigkeit des Spannungsfeldes unterschiedliche Grenzspannungen. Im Probeninneren (im ebenen Dehnungszustand) ist die Grenzspannung gegenüber der Probenoberfläche (im ebenen Spannungszustand) nach Abschätzung des von-

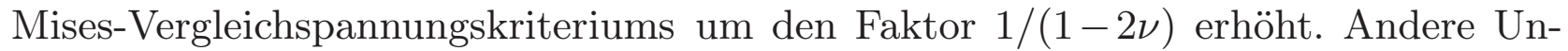
tersuchungen legen diesen Faktor zu $2^{3 / 4}=1,68$ fest $[7,27]$. Folglich ist - für das Irwin-Modell - die Ausdehnung $r_{p}$ der Zone inelastischen Materialverhaltens im ebenen Dehnungszustand (EDZ) gegenüber dem ebenen Spannungszustand (ESZ) etwa um diesen Faktor geringer. Des weiteren wird die inelastische Zone im Zusammenhang mit Gesteinen üblicherweise als Prozeßzone und nicht als plastische Zone (wie bei Metallen) bezeichnet, um der Besonderheit des Versagensverhaltens des betrachteten Materials Rechnung zu tragen: Als lokaler Versagensmechanismus dominiert bei Gesteinen Mikrorißbildung über plastischen Spannungsabbau. In der Literatur werden die Begriffe „plastische Zone“ und „Prozeßzone“ teilweise nebeneinander benutzt. Dadurch wird zwischen verschiedenen Zonen der Inelastizität unterscheiden, wobei die Prozeßzone die Anteile in unmittelbarer Nähe der Rißspitze umfaßt, wo die Bedingungen (u.a. das Auftreten großer Versatzbeträge infolge der Beanspruchung) durch die (für endliche Verformungen definierte) Plastizitätstheorie nicht mehr ausreichend genau beschrieben werden [3,5,14]. Der Begriff Prozeßzone, wie er im Rahmen dieser Arbeit verstanden wird, faßst jedoch sämtliche nichtlinearen und inelastischen (insbesondere also auch plastische) Prozesse in der Nähe der Rißspitze zusammen.

Neben dem Irwinschen gibt es weitere Modelle, die die Prozeßzone beschreiben. Das gebräuchlichste Modell ergibt sich aus dem elastischen Spannungsfeld (Gln. 1 und 3) durch Verwendung einer aus der von Mises-Vergleichsspannung abgeleiteten Versagensbedingung $\left(2 \sigma_{f}^{2}=\left(\sigma_{1}-\sigma_{2}\right)^{2}+\left(\sigma_{2}-\sigma_{3}\right)^{2}+\left(\sigma_{3}-\sigma_{1}\right)^{2}\right)$. Da in diesem Fall auch die Spannungsverteilung außerhalb des Ligaments berücksichtigt wird, ist der Prozeßzonenradius prinzipiell eine Funktion des Winkels $\varphi$. Für Modus-IBelastungsbedingungen ergibt sich (siehe z. B. [31]):

$$
\begin{aligned}
& r_{p, I}=\frac{1}{2 \pi}\left(\frac{K_{I}}{\sigma_{f}}\right)^{2} \cos ^{2} \frac{\varphi}{2}\left(1+3 \sin ^{2} \frac{\varphi}{2}\right) \\
& r_{p, I}=\frac{1}{2 \pi}\left(\frac{K_{I}}{\sigma_{f}}\right)^{2} \cos ^{2} \frac{\varphi}{2}\left[1+3 \sin ^{2} \frac{\varphi}{2}-4 \nu(1-\nu)\right]
\end{aligned}
$$

und für Modus-II-Belastungsbedingungen:

$$
\begin{aligned}
& r_{p, I I}=\frac{1}{2 \pi}\left(\frac{K_{I I}}{\sigma_{f}}\right)^{2}\left(3-\frac{9}{4} \sin ^{2} \varphi+\sin ^{2} \frac{\varphi}{2}\right), \\
& r_{p, I I}=\frac{1}{2 \pi}\left(\frac{K_{I I}}{\sigma_{f}}\right)^{2}\left[3-\frac{9}{4} \sin ^{2} \varphi+\sin ^{2} \frac{\varphi}{2}(1-2 \nu)^{2}\right] .
\end{aligned}
$$

Eine graphische Darstellung dieser Beziehungen ist in Abb. 5 gegeben. 

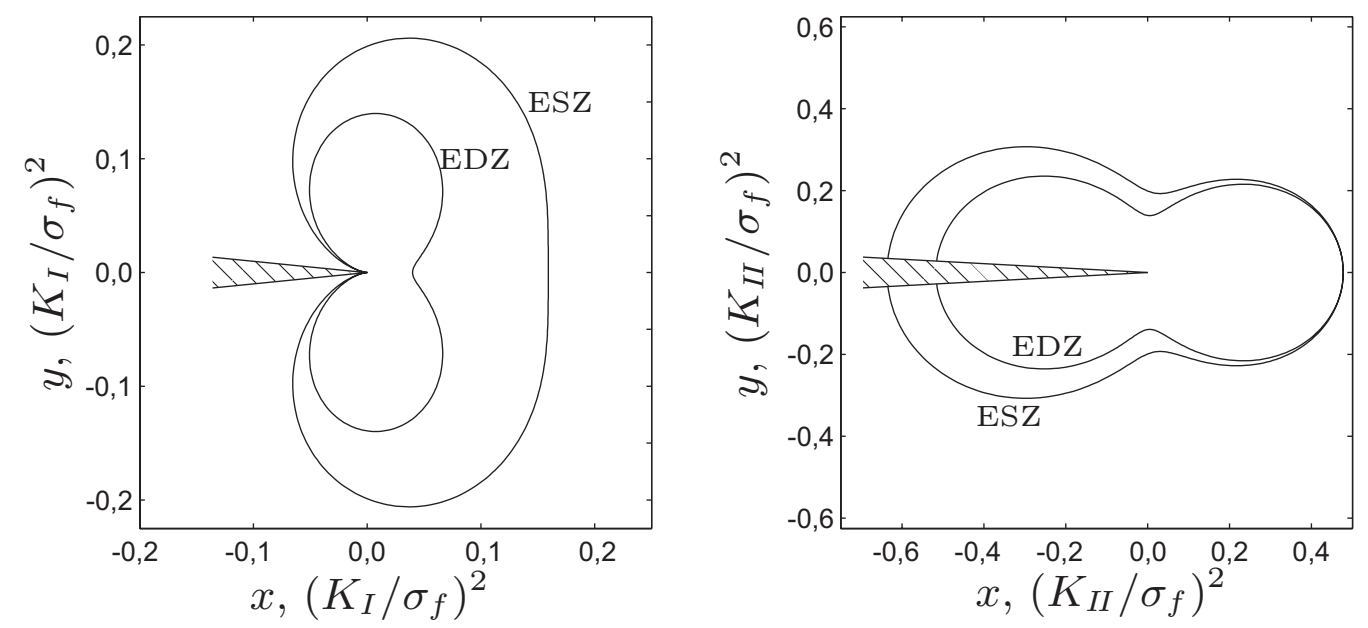

Abb. 5: Prozeßzonen für Modus-I (links) und Modus-II (rechts) unter äquivalenten Belastungsbedingungen $K_{I}=K_{I I}(\nu=1 / 4)$.

Beide vorgestellten Modelle weisen eine lineare Proportionalität zwischen der Prozeßzonengröße und dem Quadrat des Spannungsintensitätsfaktors (und somit mit der Bruchenergie, s. Abs. 2.1.5) auf. Darüber hinaus besteht eine invers quadratische Proportionalität zur Grenzspannung $\left(r_{p} \sim K^{2} / \sigma_{f}^{2}\right)$. Sowohl der Spannungsintensitätsfaktor als Maß für die Gefährlichkeit des Risses als auch der Festigkeitswert bestimmen das Versagensverhalten entscheidend. Diese beiden Größen legen die Größe der Prozeßzone und somit die Grenze des Bereiches fest, in dem linear-elastische Gesetzmäßigkeiten gültig sind. Sie werden deshalb dazu benutzt, die Anwendbarkeit der linear-elastischen Bruchmechanik zu prüfen und für Laboruntersuchungen die Mindestprobenabmessungen festzulegen (wie im folgenden Abschnitt beschrieben). Die Annahme ebener Zustände stellt eine Vereinfachung dar. Im Dreidimensionalen ergeben sich (für Grenzfälle sehr dünner oder sehr dicker Körper sogar fundamentale) Änderungen von Form und Größe der Prozeßzone [32].

Untersuchungen der mikromechanischen Vorgänge in der Prozeßzone sowie die Ermittlung der Prozeßzonengröße mit experimentellen Methoden sind kompliziert (ein ausführlicher Überblick findet sich in [33], s. auch [34]). Die mikromechanischen Vorgänge bei der Energiedissipation in der Prozeßzone werden jedoch für die Bestimmung von Bruchzähigkeiten nicht im Detail studiert, obwohl sie ihre Stärke entscheidend bestimmen und implizit das Ausbreitungsverhalten von Rissen (Abschnitt 2.2) beeinflussen. Das dissipative Gebiet der Prozeßzone wird als „black box“ betrachtet, es muß lediglich sichergestellt werden, daß die Interpretation im Rahmen der linearelastischen Bruchmechanik außerhalb dieser Zone erfolgt (s. folgenden Abschnitt). 


\subsubsection{Gültigkeitsgrenzen der linear-elastischen Bruchmechanik}

Einer der wesentlichen Aspekte bei der Anwendung der linear-elastischen Bruchmechanik ist es, zur Bewertung der Sicherheit von Strukturen vergleichbare, größenunabhängige Kennwerte bestimmen zu können, die das Bruchverhalten eines Materials eindeutig beschreiben und somit einen einzigen charakteristischen Materialparameter liefern. Für die Bestimmung solcher Kennwerte im Sinne konservativer Sicherheitserwägungen muß die bruchmechanische Situation mit dem größten Gefährdungspotential zugrunde gelegt werden. Dieses ist für den Spannungszustand gegeben, für den die kleinste Zone nichtlinearer Deformationen resultiert. Im allgemeinen ist dies die Zug(Modus-I)-Belastung im ebenen Dehnungszustand, d. h. der für Sicherheitsaspekte relevante Rißinitiierungskennwert ist die Bruchzähigkeit $K_{I c}$ (englische Bezeichnung ,plane strain fracture toughness“). Die an die Bestimmung von gültigen Bruchzähigkeiten geknüpften Bedingungen wurden von der „American Society for Testing and Materials" (ASTM - Amerikanische Materialprüfungsgesellschaft) in Form von Prüfvorschriften zur $K_{I c}$-Bestimmung in Laborexperimenten eingeführt (siehe z. B. ASTM E 399 [35]). Ein Kernpunkt der Vorschriften ist die Festlegung von Mindestprobengrößen. Diese sind mit der Größe der Prozeßzone korreliert, wie im folgenden erläutert.

Die laterale Ausdehnung der Rißspitzenprozeßzone ist - wie im vorangegangenen Abschnitt beschrieben - über den Querschnitt einer Struktur nicht konstant. Die Rißspitzenprozeßzone ist an der Oberfläche (im ebenen Spannungszustand) größer als im Inneren (im ebenen Dehnungszustand). In Abb. 6 ist dies für das durch Gl.(8) bestimmte Modell am Beispiel einer zugbelasteten ebenen Scheibe gezeigt. Wegen der Form der Prozeßzone wird dieses Modell auch als Hundeknochenmodell bezeichnet. Der sich unter der Oberfläche ausbildende Übergangsbereich ist von endlicher Ausdehnung, da er auf den Bereich beschränkt ist, in dem sich die Reduktion der
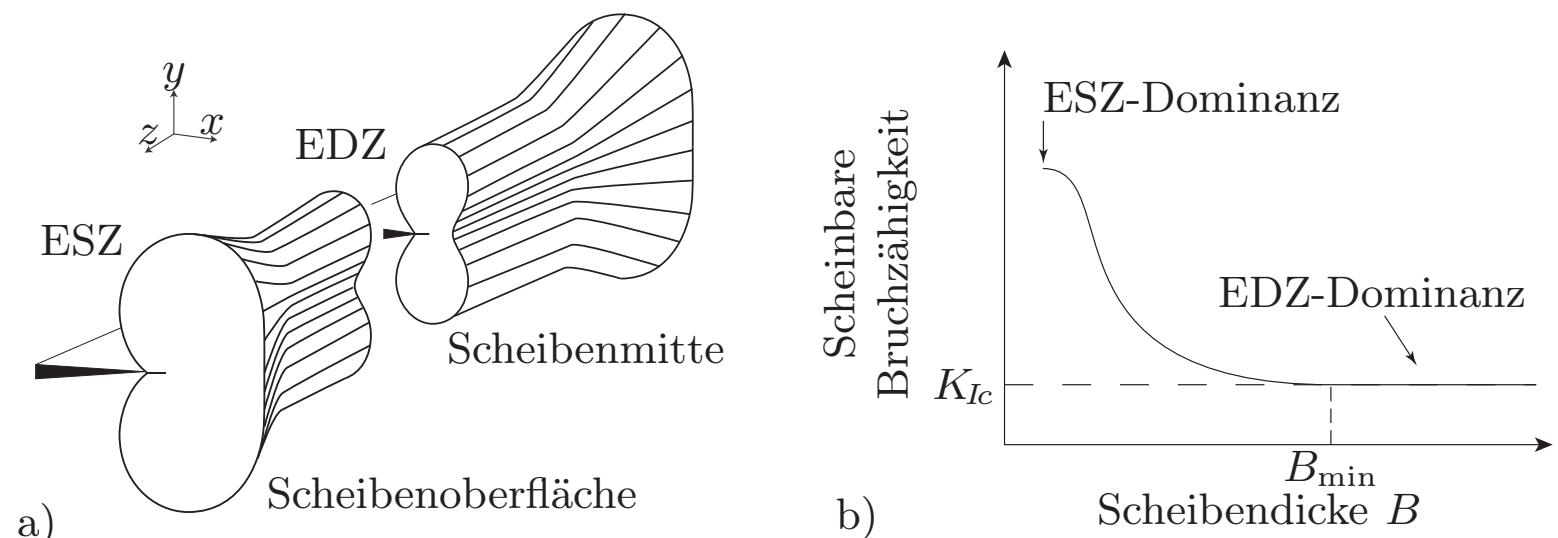

Abb. 6: Zusammenhang zwischen Scheibendicke und Prozeßzonengröße bzw. Bruchwiderstand (schematisch): a) Hundeknochenmodell der Prozeßzone, b) Bruchzähigkeit als Funktion der Scheibendicke. 
Mehrachsigkeit auswirkt. Infolgedessen entsteht ab einer gewissen Scheibendicke im Scheibeninneren ein Bereich mit konstant geringer Prozeßzonengröße und konstant geringem Bruchwiderstand. Für dicke Scheiben läßt sich daher der Gesamtzustand näherungsweise dadurch beschreiben, daß für die gesamte Scheibe das Vorliegen eines ebenen Dehnungszustand angenommen wird, d.h. der ebene Dehnungszustand dominiert. Demgegenüber kann sich in dünnen Scheiben kein ebener Dehnungszustand ausbilden und das Gesamtverhalten läßt sich näherungsweise durch den ebenen Spannungszustand beschreiben. Bezogen auf die Ermittlung eines größenunabhängigen Rißinitiierungskennwertes bedeutet dies, daß die Scheibendicke hinreichend groß sein muß, damit die verfälschenden Oberflächeneffekte vernachlässigt werden können. Wie in Abb.6b skizziert, werden bis zu einer Mindestscheibendicke $\left(B_{\min }\right)$ scheinbare Bruchzähigkeiten bestimmt, die den materialspezifischen Bruchwiderstand überschätzen.

Darüber hinaus muß die Größe der Prozeßzone, in der linear-elastische Grundsätze keine Gültigkeit besitzen, vernachlässigbar klein sein im Verhältnis zu sämtlichen anderen relevanten Dimensionen der betrachteten Struktur. Dieses sogenannte Kleinbereichsfließen (,small scale yielding“6) ist eine grundlegende Forderung für die Anwendbarkeit des K-Konzeptes. Dadurch wird gewährleistet, daß der Gesamtzustand der Struktur auf linear-elastischen Gesetzmäßigkeiten beruht und das Verhalten ausserhalb der Prozeßzone durch die Spannungsintensitätsfaktoren (Gl.3) bzw. durch die allgemeine Lösung des Spannungsfeldes (Gl.5) bestimmt wird. Die Verletzung dieser Forderung führt ebenfalls zu einer fehlerhaften Bestimmung bruchmechanischer Kennwerte.

Auf der Basis der vorstehend aufgeführten Bedingungen legt die o.a. ASTMPrüfvorschrift E 399 die Mindestprobengrößen für Laborexperimente zu:

$$
a, B,(W-a) \geq 2,5\left(\frac{K_{I}}{\sigma_{f}}\right)^{2}
$$

fest. Hierbei bezeichnet $B$ die Probendicke; die Rißlänge $a$ sowie die Länge des Ligaments $(W-a)$ legen den Abstand der Rißspitze zur Probenberandung fest. Der Vergleich mit den Gln. (7) und (8) zeigt, daß die Mindestprobengröße quantitativ über den Faktor $\left(K_{I} / \sigma_{f}\right)^{2}$ mit der Prozeßzonengröße zusammenhängt.

Ähnliche Prüfvorschriften zur Bestimmung von Scherbruchzähigkeiten (Modus-II oder -III) wurden bislang nicht erlassen. Auch bei Vorliegen einer anderen als einer reinen Zug(Modus-I)-Beanspruchung müssen die vorstehenden Anforderungen beachtet werden und die Gültigkeitsbedingungen entsprechend angepaßt werden. Nach einer Untersuchung von Hiese [31,36] resultieren beispielsweise für Scher(Modus-II)Experimente andere Mindestprobengrößen. Für diesen Fall muß die Probengröße bezüglich der Probenebene um einen Faktor vier größer sein, bezüglich der Probendicke sind aber Proben zulässig, die um einen Faktor drei dünner sind als unter Zug(ModusI)-Beanspruchung. Da Bruchwiderstände, entgegen der allgemeinen Annahme, unter 
Umständen bei Scher(Modus-II)-Beanspruchung oder bei gemischter Beanspruchung geringere Werte annehmen können als bei Zug(Modus-I)-Beanspruchung (s. auch Abs. 2.2), wurde ein Hinweis auf die geänderten Gültigkeitsanforderungen in die neueste Auflage der FKM-Richtlinie „Bruchmechanischer Festigkeitsnachweis“ [37,38] aufgenommen.

Die hier geschilderten Einschränkungen betreffen ausschließlich die Bestimmung von kritischen Spannungsintensitätsfaktoren im ebenen Dehnungszustand. Die Anwendung der linear-elastischen Bruchmechanik zur Bestimmung anderer Rißspitzenparameter bleibt davon unberührt, solange die Bedingung des Kleinbereichsfließens gilt. Solche bruchmechanischen Größen können vergleichend auf Strukturen gleicher Dicke und gleichen Materials übertragen werden. Sobald jedoch das Kleinbereichsfließen nicht mehr gewährleistet ist, sollten andere bruchmechanische Methoden, wie das $J$-Integral-Konzept, angewendet werden.

\subsubsection{Energieansatz - das Griffithsche G-Konzept}

Alternativ zu dem vorstehend beschriebenen $K$-Konzept kann das Bruchkriterium auch über einen Energieansatz formuliert werden. Die Definition dieses Bruchkriteriums geht auf Arbeiten von A. A. Griffith [39,40] zurück und markiert durch die erstmalige Berücksichtigung von Rissen bei der Versagensanalyse den Beginn der Bruchmechanik.

In diesem Energieansatz beschreibt die Energiefreisetzungsrate $G$ die Beanspruchung eines Risses in einem belasteten Körper. Sie ist gegeben als die bei einer gedachten, infinitesimalen Vergrößerung der Bruchfläche resultierende Abnahme der potentiellen, elastischen Energie $\mathrm{d} U_{p}$ des Körpers und damit freiwerdenden, für eine eventuelle Rißausbreitung zur Verfügung stehende Energie. Für ebene Scheiben der Dicke $B$ und Rißverlängerung um $\mathrm{d} a$ ist:

$$
G=-\frac{1}{B} \frac{\mathrm{d} U_{p}}{\mathrm{~d} a} .
$$

Je nach Art der Beanspruchung der Probe werden zwei idealisierte Extremfälle unterschieden: Für den Fall konstanter Verschiebung $(l=$ const., „Fixed Grip“, siehe Abb. 7a) ist die Änderung der potentiellen, elastischen Energie allein gegeben durch die Änderung der inneren, in der Probe gespeicherten Formänderungsenergie $\mathrm{d} U_{p}^{i}$, $\mathrm{d} U_{\mathrm{p}}=\mathrm{d} U_{p}^{i}$. Für den Fall konstanter Last $(P=$ const., „Fixed Load“, siehe Abb. 7b) ist zusätzlich die von den Belastungskräften geleistete äußere Arbeit $\mathrm{d} W^{a}$ zu berücksichtigen, $\mathrm{d} U_{p}=\mathrm{d} U_{p}^{i}-\mathrm{d} W^{a}=-\mathrm{d} U_{p}^{i}$, mit $W^{a}=2 U_{p}^{i}$. Allgemein ist:

$$
G=-\frac{1}{B} \frac{\mathrm{d} U_{p}}{\mathrm{~d} a}=-\frac{1}{B} \frac{\mathrm{d}}{\mathrm{d} a}\left(U_{p}^{i}-W^{a}\right),
$$

mit: $\quad W^{a}=0$ für konstante Verschiebung

$W^{a} \neq 0$ für konstante Last. 

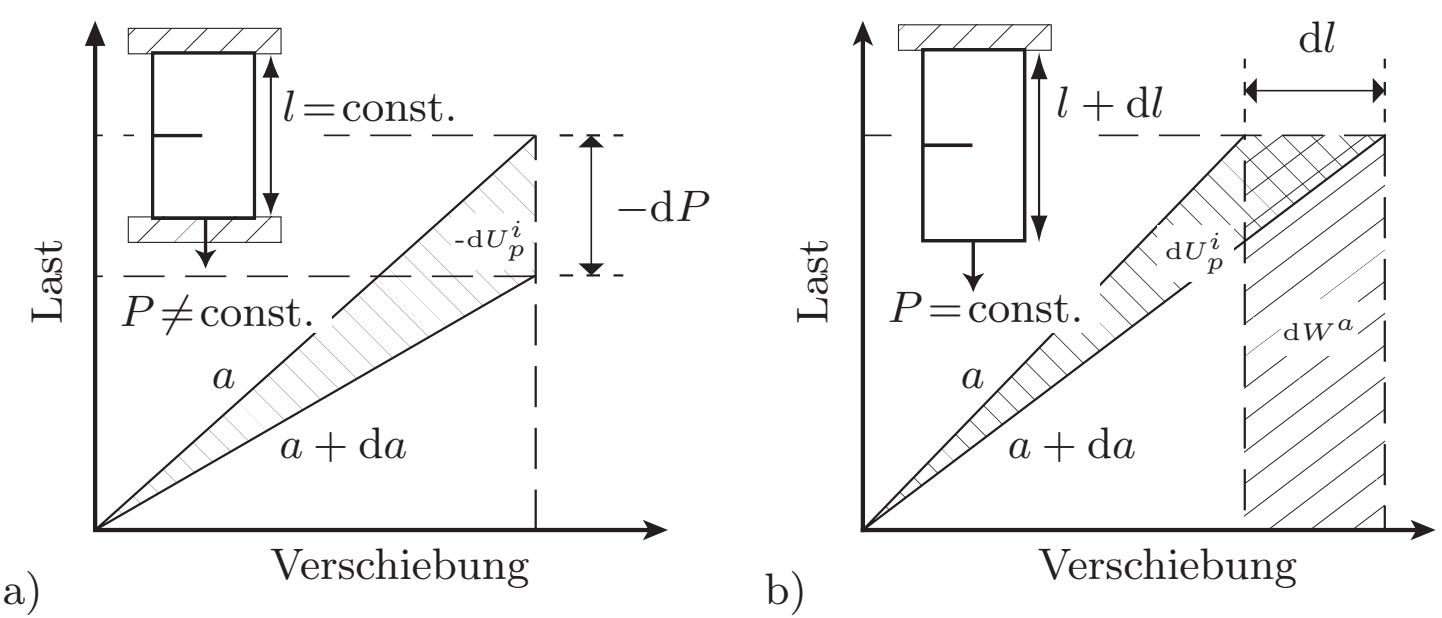

Abb. 7: Energieänderung bei Rißverlängerung um da für: a) konstante Verschiebung $(\mathrm{d} l=0)$ und $\mathrm{b})$ konstante Last $(\mathrm{d} P=0)$, nach [1].

Entspricht die Abnahme der potentiellen, elastischen Energie und damit die für Rißausbreitung zur Verfügung stehende Energie der bei der Erzeugung der neuen Bruchfläche dissipierten Energie $U_{d}$ tritt Bruchausbreitung ein, der Zustand wird kritisch mit $G=G_{c}$, wobei $G_{c}$ die spezifische Bruchenergie bezeichnet:

$$
\frac{\mathrm{d}}{\mathrm{d} a}\left(U_{p}+U_{d}\right)=0
$$

Zwischen der Energiefreisetzungsrate und den Spannungsintensitätsfaktoren besteht eine vollständige Analogie. Dies zeigte Irwin [11,41] über die Berechnung der Rißschließenergie. Die Rißschließenergie $U_{p}^{s}\left(U_{p}^{s}=-U_{p}\right)$ ist die Energie, die benötigt wird, einen um d $a$ verlängerten Riß auf der gleichen Länge wieder zu schließen (Abb. 8). Die Rißschließenergie ist gegeben durch:

$$
\mathrm{d} U_{p}^{s}=2 \int_{a}^{a+\mathrm{d} a} \frac{1}{2} \sigma_{y} \widetilde{v}_{r} \mathrm{~d} x
$$

mit $\widetilde{v}_{r}$ (Rißuferverschiebung vor dem Rißschließen) entsprechend Gl. (2) für $\varphi= \pm \pi$ und allgemeine Spannungsintensitätsfaktoren. Daraus ergibt sich mit Gl. (11) folgende Beziehung zwischen Energiefreisetzungsrate und Spannungsintensitätsfaktor:

$$
G_{I, I I}=\frac{K_{I, I I}^{2}}{E^{*}}
$$

mit: $\quad E^{*}= \begin{cases}E & \text { für ESZ } \\ E /\left(1-\nu^{2}\right) & \text { für EDZ }\end{cases}$ 
a)

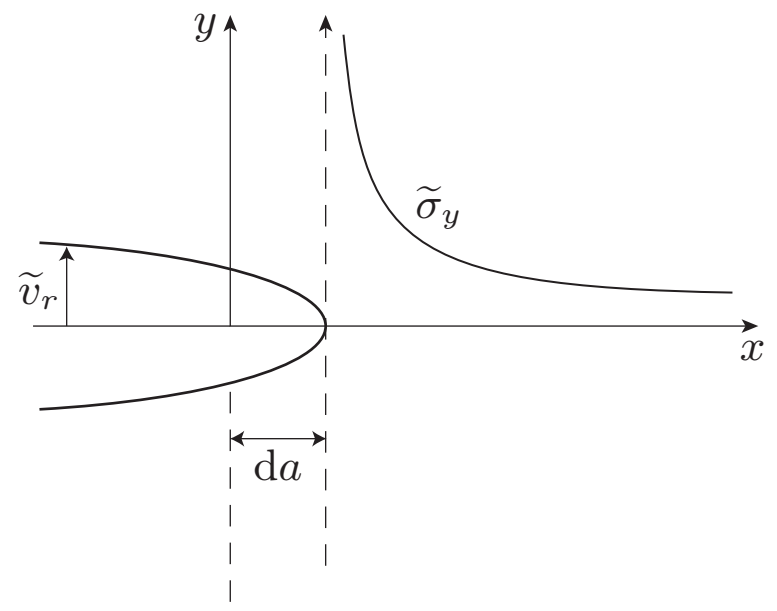

b)

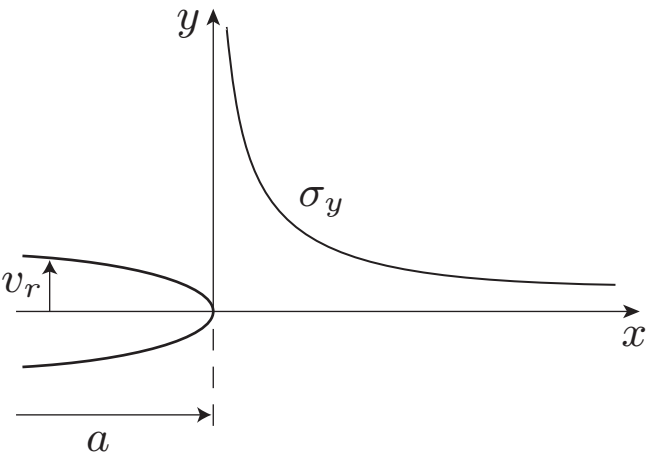

Abb. 8: Spannungsverteilung $\widetilde{\sigma}_{y}$ bzw. $\sigma_{y}$ und Öffnung $\widetilde{v}_{r}$ bzw. $v_{r}$ eines Risses: a) vor und b) nach Rißschließen um da.

und

$$
G_{I I I}=\frac{K_{I I I}^{2}(1+\nu)}{E} .
$$

Bei gemischter Beanspruchung ergibt sich im Energie- $G$-Bild die gesamte Energiefreisetzungsrate (im Gegensatz zum Spannungs- $K$-Bild - s. o.) direkt aus der Summe der Beiträge der einzelnen Moden: $G=G_{I}+G_{I I}+G_{I I I}$.

Für das Einsetzen von Rißinstabilität ist Gl. (15) entsprechend anzuwenden auf die Bruchzähigkeit $K_{c}$ und die spezifische Bruchenergie $G_{c}$, die den kritischen Wert der Energiefreisetzungsrate darstellt. Die spezifische Bruchenergie $G_{c}$ charakterisiert hierbei den materialspezifischen Grenzwert, ab dem die durch die Energiefreisetzungsrate beschriebene Energieanlieferung ausreicht, um den für die Rißausbreitung benötigten Energiebedarf zu decken. In Erweiterung der ursprünglichen Formulierung durch Griffith, bei der nur Beiträge der Oberflächenenergie (an der neu geschaffenen Bruch,,ober"fläche gespeicherte Energie) zur Energiedissipation berücksichtigt wurden, umfaßt die spezifische Bruchenergie - wegen der Äquivalenz des $K$ - und des $G$-Konzeptes - die gesamte während der Rißausbreitung in der Prozeßzone dissipierte Energie. Ein Riffortschritt um da wird demnach durch die Verschiebung der 
Prozeßzone um diesen Betrag beschrieben, die, wie in Abb. 9 für einen Modus-I-Riß skizziert, dabei eine Zone irreversibler Materialschädigung entlang der Rißufer hinterläßst. Durch diese Sichtweise ist es möglich den Energieansatz, anders als ursprünglich angenommen (Griffith schränkte die Anwendbarkeit seiner Theorie auf Materialien - wie z. B. Glas - ein, die sich streng linear-elastisch verhalten), auf eine große Zahl von Materialien zu beziehen (siehe auch [42-44]).

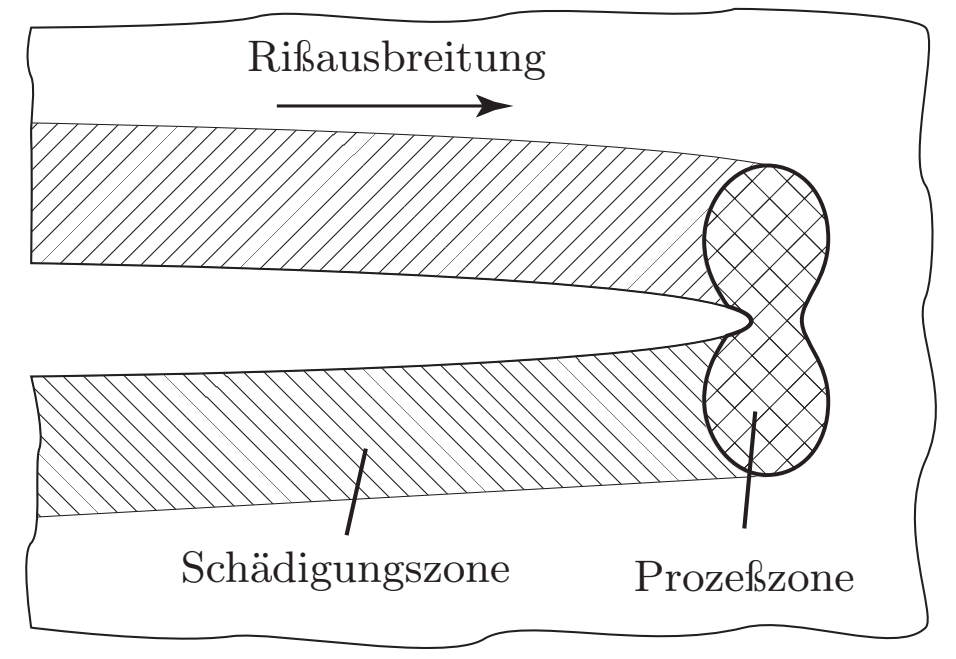

Abb. 9: Ausbildung einer Schädigungszone bei Rißausbreitung.

\section{$2.2 \quad$ Bruchausbreitung}

\subsubsection{Bruchausbreitungskriterium}

Die Bruchausbreitung durch Rißverlängerung kann stabil oder instabil verlaufen. Bei stabiler Rißverlängerung besteht die Möglichkeit den Ausbreitungsvorgang zu kontrollieren, bei instabiler Rißverlängerung hingegen im allgemeinen nicht. Die in den vorangegangenen Abschnitten vorgestellten Initiierungskriterien beziehen sich auf den Beginn instabiler Rißverlängerung. Diese tritt bei Überschreiten der Bruchbedingung $\left(K \geq K_{c}\right.$ bzw. $G \geq G_{c}$ ), ab dem Erreichen einer bestimmten kritischen Kombination aus Belastung $\sigma_{c}$ und Rißlänge $a_{c}$ ein. Abbildung 10 zeigt dies für das Spannungs-K-Bild (Abb. 10a) und für das Energie-G-Bild (Abb. 10b) anhand des einfachen Beispiels aus Abschnitt 2.1.1. Dargestellt sind Beanspruchungskurven als Funktion der Rißlänge für jeweils drei Laststufen. Da bei der Spannung $\sigma_{01} \mathrm{im}$ betrachteten Rißlängenintervall die Bruchbedingung nicht erfüllt ist (kein Schnittpunkt zwischen den Beanspruchungskurve und kritischem Rißinitiierungskennwert), tritt für diese Laststufe keine Rißverlängerung auf. Für die nächst höhere Laststufe $\sigma_{02}$ ist die Rißinitiierungsbedingung für alle Risse, deren Länge größer als $a_{c 2}$ ist, 


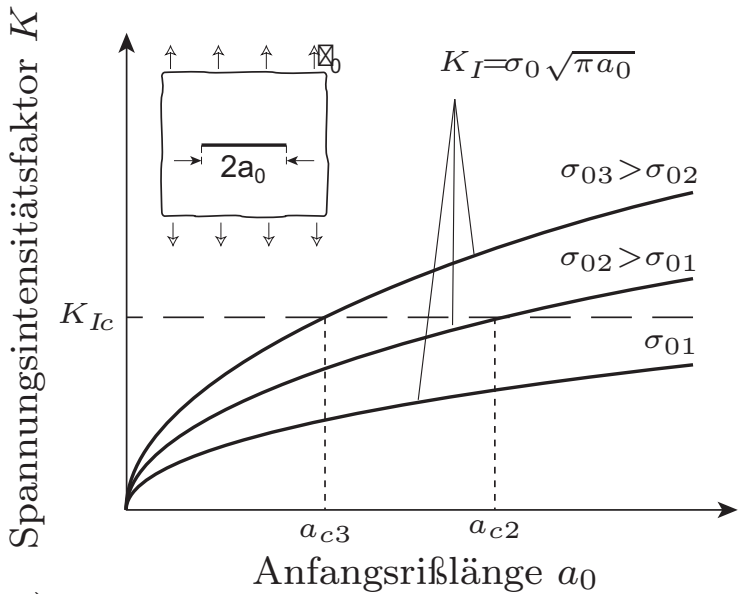

a)

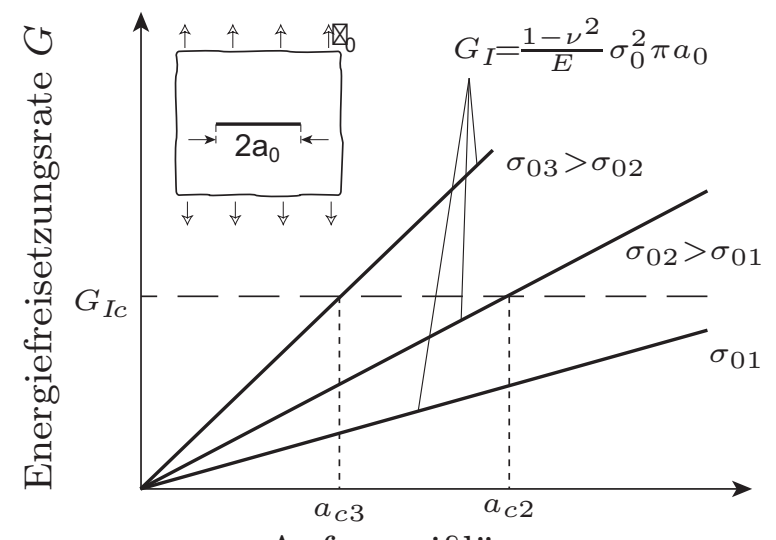

b)

Abb. 10: Rißinitiierung im a) Spannungs- $K$-Bild und b) Energie-G-Bild (nach [45]).

erfüllt. Bei der höchsten der drei Laststufen $\sigma_{03}$ werden sich alle Risse, die länger als $a_{c 3}$ sind, instabil ausbreiten. Dabei besteht nicht nur zwischen dem Spannungsintensitätsfaktor und der Energiefreisetzungsrate bei der Beschreibung der Beanspruchung eine Analogie zwischen Spannungs- $K$-Bild und Energie- $G$-Bild, sondern auch im Bezug auf die kritischen Werte der Beanspruchung, die den Widerstand des Materials gegen Rißverlängerung wiedergeben. Daher werden diese kritischen Werte im folgenden zusammenfassend als Rißwiderstand $R$ bezeichnet.

Bei Bruchausbreitungsvorgängen muß die Bruchbedingung nicht nur für die erstmalige Initiierung des stationären Risses, sondern auch für die weitere Rißverlängerung erfüllt sein. Dabei ist das Bruchverhalten, d. h. die Beziehung zwischen dem Rißwiderstand und der Rißinitiierung bzw. der Rißverlängerung materialabhängig. Das unterschiedliche Bruchverhalten findet Ausdruck in der Form der Rißwiderstandskurve oder $R$ - $\Delta a$-Kurve (kurz: $R$-Kurve), die den Rißwiderstand bezüglich der Rißverlängerung beschreibt. Ein Material, für das nach der Rißinitiierung bei fortschreitender Rißverlängerung stets derselbe Energiebetrag verbraucht wird, zeichnet sich durch eine „flache" $R$-Kurve aus. Für ein solches Material ist der Rißwiderstand eine Konstante: $R=K_{c}$ bzw. $R=G_{c}$. Dieses Bruchverhalten ist in Abb. 11a dargestellt. Die Kurve, die $R$ als Funktion der Rißlänge beschreibt, weist nach dem sprunghaften Anstieg bei $a=a_{c}$ keine weitere Steigerung auf. Dieses Verhalten bzw. eine solche Form der $R$-Kurve ist charakteristisch für Materialien mit sprödem Bruchverhalten, die Rißausbreitung erfolgt sofort nach der Initiierung und somit für den gesamten Ausbreitungsprozeß instabil. Im Gegensatz dazu tritt bei stärker plastischen Materialien mit stärker duktilem Bruchverhalten stabile subkritische Rißverlängerung vor dem Einsetzen der instabilen Bruchausbreitung auf, wie in Abb. 11b dargestellt. Bei solchen Materialien steigt die $R$-Kurve nach dem Beginn der Rißausbreitung an. Bei fortschreitender Rißverlängerung wird ein immer größerer Energiebetrag verbraucht, d. h. der Rißwiderstand ist nicht konstant: $R=R(a)$. Dies ist darauf zurück- 


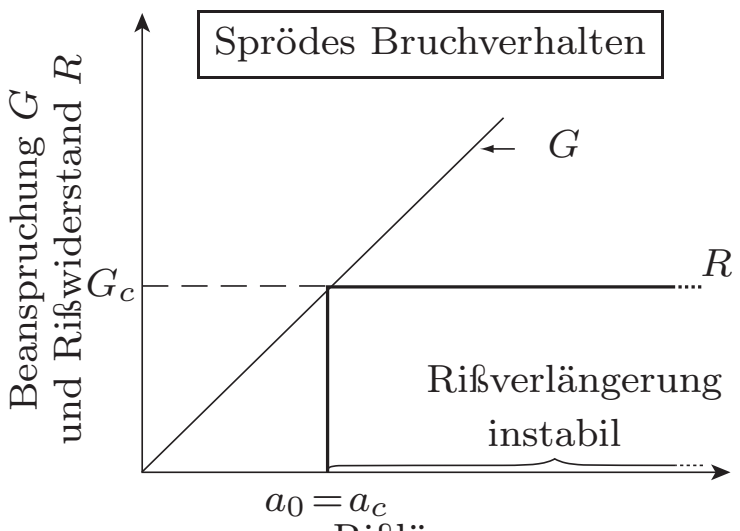

a)

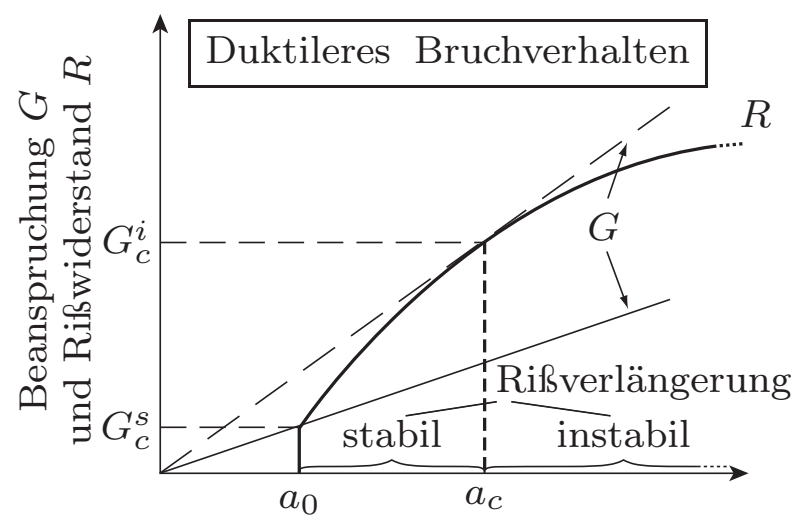

b)

Rißlänge $a$

Abb. 11: Beanspruchung $(G)$ und Rißwiderstand $(R)$ für: a) flache Rißwiderstandskurve und b) steigende Rißwiderstandskurve bei Rißverlängerung.

zuführen, daß die Größe der Prozeßzone bei der Rißverlängerung zunimmt. Dieses Verhalten ist typisch für viele metallische Materialien [46]. In der Abbildung sind zwei Rißinitiierungswerte gekennzeichnet: Hierbei bezeichnet $G_{c}^{s}$ (dem entspräche $K_{c}^{s}$ im Spannungs- $K$-Bild) den Beginn der stabilen Rißausbreitung; Das Einsetzen der instabilen Rißausbreitung, bezeichnet mit $G_{c}^{i}\left(K_{c}^{i}\right)$, erfolgt dort, wo die Beanspruchungskurve eine Tangente an die $R$-Kurve bildet. Da das Bruchverhalten eines solchen Materials von der Rißlänge abhängt, kann es nicht durch einen einzelnen charakteristischen Kennwert beschrieben werden, sondern wird vielmehr durch den gesamten Verlauf der $R$-Kurve bestimmt. Häufig wird jedoch trotzdem nur der Bruchzähigkeitswert für den Beginn der stabilen Rißverlängerung $K_{c}^{s}$ bestimmt, weil die Bestimmung der gesamten $R$-Kurve eines Materials sehr aufwendig ist.

Insgesamt wird zur Analyse von Rißausbreitungsphänomenen neben der notwendigen Bedingung, daß das Initiierungskriterium

$$
K \geq K_{c}=R \quad \text { bzw. } \quad G \geq G_{c}=R^{*}
$$

erfüllt ist, eine hinreichende Bedingung benötigt, die aus der Monotonie der Beanspruchungskurve abgeleitet wird. Für die instabile Rißausbreitung muß bezüglich der Rißverlängerung um d $a$ die Beanspruchung relativ zum Rißwiderstand ansteigen:

$$
\frac{\mathrm{d} K}{\mathrm{~d} a}>\frac{\mathrm{d} R}{\mathrm{~d} a} \quad \text { bzw. } \quad \frac{\mathrm{d} G}{\mathrm{~d} a}>\frac{\mathrm{d} R}{\mathrm{~d} a} .
$$

Wohingegen Risse sich stabil ausbreiten wenn:

$$
\frac{\mathrm{d} K}{\mathrm{~d} a} \leq \frac{\mathrm{d} R}{\mathrm{~d} a} \quad \text { bzw. } \quad \frac{\mathrm{d} G}{\mathrm{~d} a} \leq \frac{\mathrm{d} R}{\mathrm{~d} a} .
$$

Andere geläufige Bezeichnungen für $R$ lauten im Spannungs- $K$-Bild $K_{R}$ oder $T$ bzw. im Energie- $G$-Bild $G_{R}$ oder $R$. 
Anders ausgedrückt wird nach der Initiierung die Rißausbreitung nur aufrechterhalten (oder sogar beschleunigt verlaufen), solange die Energieanlieferungsrate nach Gl. (17) den Materialwiderstand gegen Rißausbreitung übersteigt Ist dies nicht gegeben, wird die Rißgeschwindigkeit sinken und der Riß letztlich arretieren.

Die Form der $R$-Kurve ist nicht immer eine autonome Größe, d.h. sie kann unter gewissen Umständen von der Art der Belastung oder durch strukturelle Einflüsse bestimmt sein $[1,4,45]$. So ist es z. B. möglich, daß sich für ein Material, das gewöhnlich eine flache $R$-Kurve aufweist, bei der Verwendung in Strukturen geringer Dicke (keine Dominanz des ebenen Dehnungszustandes) eine steigende $R$-Kurve ausbildet. Anders als bei einer flachen $R$-Kurve muß die Beanspruchung bei einer steigenden $R$-Kurve im Bereich der stabilen Rißverlängerung nach der Initiierung weiter erhöht werden, um den Riß voranzutreiben.

Bei genauerer Betrachtung wird zwar auch bei sprödbrechenden Materialien der Anfang der Rißverlängerung nicht durch einen scharfen Knick, sondern durch einen Übergangsbereich beschrieben, dieser ist jedoch vernachlässigbar klein. Die vorstehend beschriebenen Zusammenhänge gelten für eine quasistatische Betrachtungsweise, auf dynamische Gesichtspunkte wird in Abs. 2.3 eingegangen. Darüber hinaus kann die Rißausbreitung weiteren Einflußfaktoren wie zeitlichen oder korrosiven Effekten unterliegen, auf die hier nicht weiter eingegangen wird.

\subsubsection{Bruchausbreitungsrichtung}

Unabhängig vom Belastungsmodus erfolgt die Rißausbreitung bei linear-elastischem Materialverhalten in der Regel als Zugriß [47-50]. Die Ausbreitungsrichtung des Risses und die Höhe des kritischen Wertes der Beanspruchung hängen von der Belastungsrichtung und somit vom Belastungsmodus bzw. bei gemischter Beanspruchung („,mixed mode“) vom Mischungsverhältnis der Belastungsmoden ab. Dabei bestimmt das Mischungsverhältnis den Abknickwinkel, der sich zwischen dem präexistierenden „Mutterrif“" und dem von dessen Spitze initiierten „Tochterriß“ einstellt (vgl. Abb. 12). In der Literatur finden sich eine Vielzahl von Modellen zur Vorhersage der Ausbreitungsrichtung und der kritischen Beanspruchung bei gemischter Belastung. Vergleichende Zusammenstellungen dieser Mixed-Mode-Bruchkriterien finden sich u. a. bei $[5,49,51]$.

In Bezug auf die Bestimmung der Bruchausbreitungsrichtung wird das Tangentialspannungskriterium von Erdogan und Sih [52] sehr gut durch experimentelle Befunde bestätigt [4,51]. Zur Betrachtung dieses Kriteriums empfiehlt es sich, die Spannungen an der Rißspitze (Gln. 1 u. 3) in Polarkoordinaten zu transformieren. Für eine 


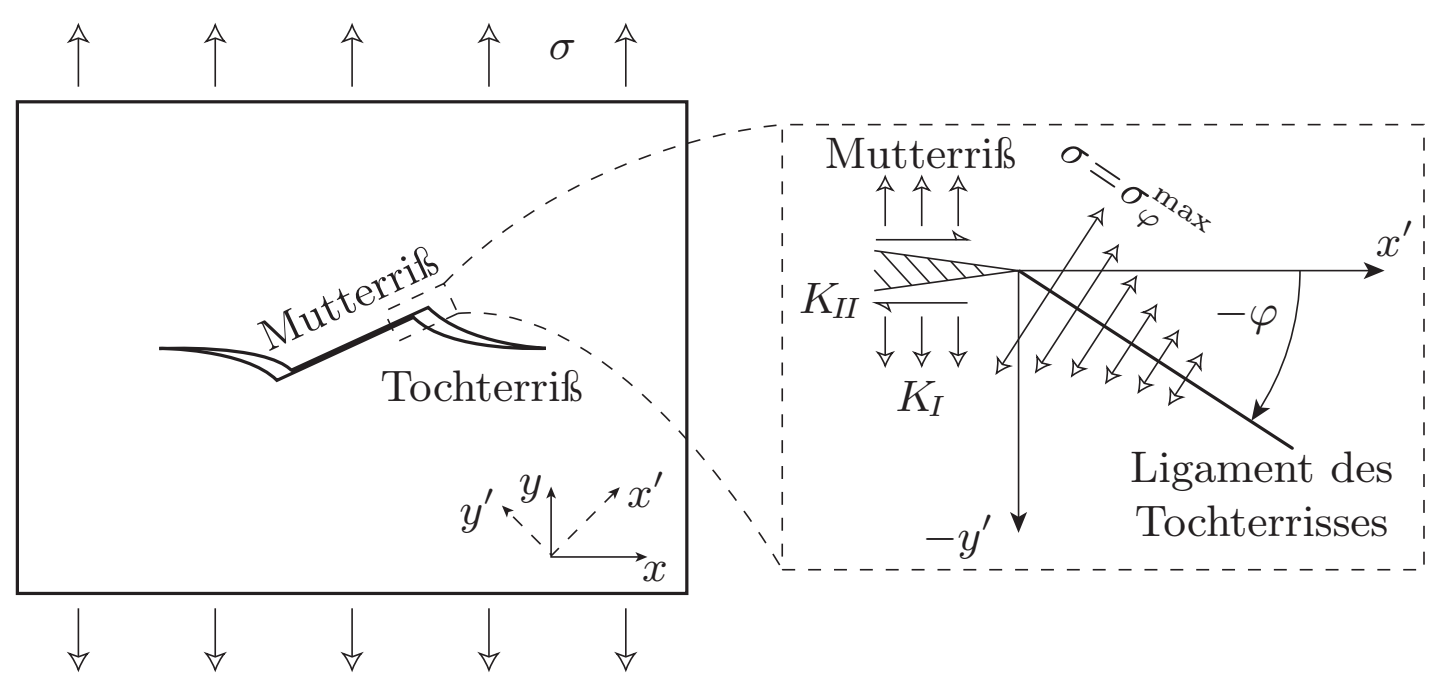

Abb. 12: Abknicken von Rissen bei Mixed-Mode-Beanspruchung.

gemischte Zug(Modus-I)- und Scher(Modus-II)-Beanspruchung lauten sie:

$$
\begin{aligned}
\sigma_{r} & =\frac{1}{2 \sqrt{2 \pi r}}\left[K_{I} \cos \frac{\varphi}{2}(3-\cos \varphi)+K_{I I} \sin \frac{\varphi}{2}(3 \cos \varphi-1)\right], \\
\sigma_{\varphi} & =\frac{1}{2 \sqrt{2 \pi r}} \cos \frac{\varphi}{2}\left[K_{I}(1+\cos \varphi)-K_{I I} 3 \sin \varphi\right], \\
\tau_{r \varphi} & =\frac{1}{2 \sqrt{2 \pi r}} \cos \frac{\varphi}{2}\left[K_{I} \sin \varphi+K_{I I}(3 \cos \varphi-1)\right] .
\end{aligned}
$$

Die Rißausbreitung erfolgt nach diesem Kriterium senkrecht zu der Richtung, in der die Tangentialspannung $\sigma_{\varphi}$ an der Spitze des Mutterrisses (vor der Initiierung) und folglich die Zugspannung für den Tochterriß (nach der Initiierung) maximal ist. Die Tangentialspannung erreicht ihr Maximum $\sigma_{\varphi}^{\max }$, wenn die deviatorischen Spannungen $\tau_{r \varphi}$ verschwinden und $\sigma_{\varphi}$ zur Hauptspannung wird. Der Abknickwinkel kann somit durch Separation aus Gl. (19) bestimmt werden. Für den Extremfall der reinen Modus-I-Beanspruchung $\left(K_{I I}=0\right)$ ergibt sich ein Winkel von $0^{\circ}$; der Riß pflanzt sich selbstähnlich fort. Für reine Modus-II-Beanspruchung $\left(K_{I}=0\right)$ ist der Betrag des Abknickwinkels $|\varphi|=70,5^{\circ}$. Das Vorzeichen des Winkels ist bei positivem Schersinn negativ (vgl. Beispiel in Abb. 12). Das hier skizzierte Kriterium kann ebenso durch Bestimmung der Richtung, in der die Energiefreisetzungsrate ihr Maximum annimmt, im Energie-G-Bild formuliert werden [4]. Zum Vergleich mit anderen Modellen bzw. experimentellen Ergebnissen sei auf die eingangs angegebenen Arbeiten verwiesen. Die mittels der verschiedenen Modelle für den Sprödbruch ermittelten Abknickwinkel sind bis zu einem Mischungsverhältnis von $50 \%$ sehr ähnlich, nur für große Anteile an Scherbeanspruchung unterscheiden sie sich geringfügig. Die Vorgänge beim Abknicken von Rissen sind Gegenstand aktueller Forschung [53, 54]. Die Richtungsstabilität der Bruchausbreitung wird durch den konstanten Term der Spannungsverteilung bestimmt [55]. 


\subsubsection{Rißinitiierung bei gemischter Beanspruchung}

Die Beschreibung der Rißinitiierung unter Mixed-Mode-Beanspruchungsbedingungen geschieht durch sogenannte Bruchgrenzkurven, die das Einsetzen instabiler Rißverlängerung in Abhängigkeit vom Mischungsverhältnis der Beanspruchung wiedergeben. Die kritische Mixed-Mode-Beanspruchung setzt sich anteilig aus Spannungsintensitätsfaktoren (SIF) der beteiligten Moden zusammen. Abbildung 13 zeigt beispielhaft eine solche Bruchgrenzkurve für eine gemischte Zug(Modus-I)- und Scher(Modus-II)-Beanspruchung. Die mit „crit.“ bezeichneten Werte sind nur mit der jeweiligen Bruchzähigkeit identisch, wenn ein reiner Belastungsmodus vorliegt - d. h. nur Belastungen eines Modus existieren -, ansonsten geben sie lediglich den Anteil des jeweiligen Modus an der Belastung wieder und es ist: $K_{I}^{\text {crit. }} \neq K_{I c}$ und $K_{I I}^{\text {crit. }} \neq K_{I I c}$. Im Allgemeinen sind die Bruchzähigkeiten unterschiedlicher Moden verschieden: $K_{\text {Ic }} \neq K_{\text {IIC }} \neq K_{\text {IIIC }}$.

Experimentell bestimmte Bruchgrenzkurven wurden von Richard und von Hiese mit bekannten Mixed-Mode-Bruchkriterien verglichen [31,51]. Dieser Vergleich zeigt, daß die Rißinitiierung unter Mixed-Mode-Beanspruchungsbedingungen stark von den charakteristischen Materialeigenschaften abhängt und die Vorhersage tatsächlicher Initiierungswerte, selbst unter Beschränkung auf Materialien mit spröden Versagenseigenschaften, nur bedingt möglich ist. Experimentelle Untersuchungen erfordern jedoch einen hohen Aufwand. Des weiteren ist festzuhalten, daß die kritischen Werte unter Zug(Modus-I)- und unter Scher(Modus-II)-Beanspruchung, teilweise erheblich, voneinander abweichen. Darüber hinaus wurde in neuesten Untersuchungen zur gemischten Beanspruchung eine Abhängigkeit von der Belastungsreihenfolge festgestellt [56].

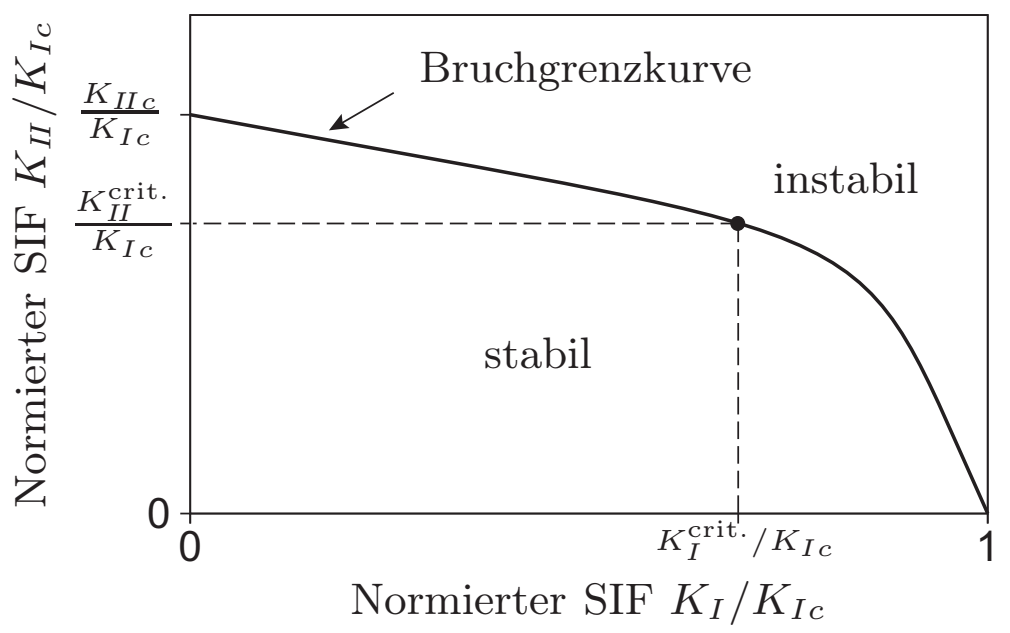

Abb. 13: Bruchgrenzkurve (schematisch) bei Rissen unter gemischter Modus-I- und Modus-II-Beanspruchung. 


\subsection{Dynamische Erweiterung der linear-elastischen Bruchme- chanik}

\subsubsection{Vorbemerkungen}

In den vorangehenden Abschnitten wurden bruchmechanische Zusammenhänge bei der Initiierung von Rissen, bei der stabilen subkritischen Bruchausbreitung sowie für den Übergang zur instabilen Bruchausbreitung vorgestellt. Für diese Fälle wird der Einfluß der Zeit und das Auftreten von Trägheitskräften, durch den dynamische Effekte gekennzeichnet sind, vernachlässigt. Dynamische Effekte gewinnen jedoch infolge hoher Belastungsraten immer größere Bedeutung je schneller die Rißausbreitung sich vollzieht [57-77]. Auch bei abrupter Beanspruchung stationärer Risse durch Schlag- oder Stoßbelastung ist eine dynamische Betrachtungsweise erforderlich [76-84]. Durch diese dynamische Betrachtungsweise, auf die bezüglich der beiden genannten Punkte im folgenden eingegangen wird, werden sowohl Effekte, die für den Beanspruchungszustand bzw. die Spannungsanalyse als auch solche, die für die materialspezifischen bruchmechanischen Parameter relevant sind, berücksichtigt.

\subsubsection{Elastische Wellen}

Die meisten dynamischen Effekte sind ursächlich mit dem Phänomen elastischer Wellenausbreitung verbunden. Die Ausbreitungsgeschwindigkeit einer Welle ist eine Materialkonstante, die von den übrigen elastischen Konstanten abhängig ist. Es wird grundsätzlich unterschieden zwischen Longitudinal- und Transversalwellen, die unterschiedliche Ausbreitungsgeschwindigkeiten besitzen. Im einfachsten Fall, bei Stabwellen, ist $v_{p}=\sqrt{E / \varrho}$ die Ausbreitungsgeschwindigkeit der Longitudinal- oder Druckwelle. Für Druckwellen ist, im Gegensatz zu Transversal- oder Scherwellen, bei der Wellenausbreitung im Raum die Dehnungsbehinderung zu berücksichtigen. Infolgedessen hängt die Longitudinal- oder Druckwellengeschwindigkeit vom Spannungszustand $\mathrm{ab}[3]$ :

$$
v_{p}=\sqrt{\frac{E}{\varrho} \frac{1-\nu}{(1+\nu)(1-2 \nu)}}, \quad \quad \text { im EDZ }
$$

und

$$
v_{p}=\sqrt{\frac{E}{\varrho} \frac{1}{1-\nu^{2}}}, \quad \quad \text { im ESZ. }
$$

Die Ausbreitungsgeschwindigkeit der Transversal- oder Scherwelle ist:

$$
v_{s}=\sqrt{\frac{\mu}{\varrho}}=\sqrt{\frac{E}{\varrho} \frac{1}{2(1+\nu)}} .
$$


Des weiteren sind für dynamische Prozesse im Rahmen der Bruchmechanik Rayleighwellen von Bedeutung. Es handelt sich hierbei um Oberflächenwellen, die sich in einem berandeten Körper/elastischen Halbraum ausbilden. Ihre Intensität nimmt exponentiell mit dem Abstand von der freien Oberfläche ab. Die Rayleighwelle besteht, in einem Verhältnis von $3 / 2$ aus einem longitudinalen und einem vertikal polarisierten transversalen Anteil. Die Partikelbewegung erfolgt auf einer retrograd durchlaufenen elliptischen Bahn, in einer Ebene senkrecht zur Oberfläche. Die Ausbreitungsgeschwindigkeit der Rayleighwelle ist mit denjenigen der anderen Wellentypen gekoppelt. Die Longitudinal- und die Transversalwellengeschwindigkeit sind ebenfalls miteinander verknüpft: Beispielsweise ist für einen Poissonschen Körper $(\nu=1 / 4)$ $v_{p} / v_{s}=\sqrt{2(1-\nu)} / \sqrt{(1-2 \nu)}=\sqrt{3} *$. Damit wird die Rayleighwellengeschwindigkeit durch ihr Verhältnis lediglich zu einer der anderen Wellengeschwindigkeiten bestimmt. In einer guten Näherung (s. [85]) wird sie gegeben durch:

$$
v_{R}=\frac{0,86+1,14 \nu}{1+\nu} v_{s}
$$

Hieraus ergibt sich, daß die Ausbreitungsgeschwindigkeit von Rayleighwellen, etwa 10\% unter der Transversalwellengeschwindigkeit liegt. Die Geschwindigkeit der Ausbreitung elastischer Wellen definiert eine obere Grenze für die Geschwindigkeit der Informationsübertragung in einem Material und somit für den Aufbau von Spannungsintensitätsfeldern.

\subsubsection{Dynamische Effekte bei stationären Rissen}

Die bei der dynamischen Beanspruchung stationärer Risse wirkenden Trägheitseffekte äußern sich dadurch, daß der Spannungsintensitätsfaktor zeitabhängig wird: $K=K(t)$. Bei dem betrachteten Problem eines Risses in Materie handelt es sich um ein schwingfähiges System mit einer charakteristischen Übertragungsfunktion. Durch eine dynamische Änderung des Beanspruchungszustandes - etwa durch Stoßoder Schlagbelastung - wird dieses System zu erzwungenen Schwingungen angeregt. Dies sei an dem einfachen Beispiel eines Risses der Länge $a$, ohne Berücksichtigung weiterer Randbedingungen, illustriert. Auf den Riß werde, wie in Abb. 14a skizziert, über einen endlichen Zeitraum $\Delta t$ eine Fernfeldspannung $\sigma_{0}$ aufgebracht und dann konstant gehalten. Bei der Antwortfunktion des Systems, gegeben durch den Aufbau des Spannungsintensitätsfeldes an der Rißspitze (Abb. 14b), sind verglichen mit der anregenden Funktion Veränderungen in Amplitude und Phase zu beobachten: Der Aufbau des Spannungsintensitätsfeldes erfolgt mit einer zeitlichen Verzögerung und die Amplitude oszilliert gedämpft um den neuen Gleichgewichtszustand $K(\infty)$. Nach

*Die Bezeichnung der Wellengeschwindigkeiten mit $v_{p}$ und $v_{s}$ folgt der in der Seismologie üblichen Terminologie: Die Longitudinalwelle wird als Primär- bzw. P-Welle (auch „pressure wave“) bezeichnet, da sie aufgrund der größeren Ausbreitungsgeschwindigkeit nach einem Erdbeben zuerst am Ort des Seismometers eintrifft, folglich wird die Transversalwelle als Sekundär- bzw. S-Welle (auch ,shear wave“) bezeichnet. 


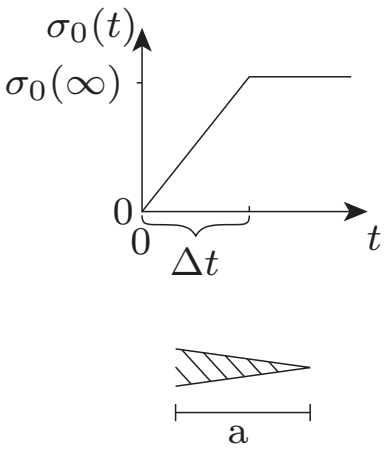

a)

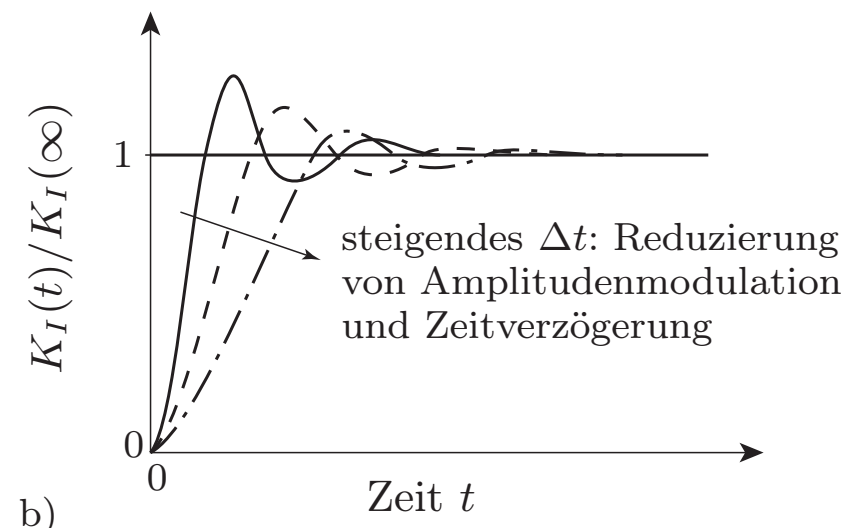

b)

Abb. 14: Aufbau des Spannungsintensitätsfeldes bei der dynamischen Belastung eines stationären Risses (nach [5]).

dem Abklingen der Schwingungen ist der Spannungsintensitätsfaktor für die statische und die dynamische Betrachtungsweise identisch. Insbesondere die Amplitudenmodulation ist für die Bruchmechanik von besonderer Bedeutung, da, im Vergleich zu der statischen Betrachtungsweise, durch das „Überschwingen“ kurzzeitig deutlich höhere Spannungsintensitätsfaktoren resultieren. Diese beiden Effekte hängen von der Belastungsrate ab, d.h. im vorliegenden Beispiel von der Länge des Zeitraumes in dem der Spannungsstoß erfolgt. Für den Übergang zu einem Rechteckpuls (,step function"), also für $\Delta t \rightarrow 0$, ist sowohl die zeitliche Verzögerung als auch die Amplitude der Überschwinger am größten. Realistisch betrachtet erfolgen die höchst möglichen Beanspruchungsraten jedoch aus einer Wellenbelastung, für die die Anstiegszeiten immer noch endlich sind. Nimmt $\Delta t$ hingegen $\mathrm{zu}$, verringert sich der Einfluß dynamischer Effekte zunehmend und wird für $\Delta t v_{s} / a \gtrsim 1$ vernachlässigbar klein [5].

Eine analytische Darstellung des dynamischen Spannungsintensitätsfeldes ist nur für wenige Spezialfälle möglich [5,69,70]. Die Bestimmung dynamischer Spannungsintensitätsfaktoren erfolgt daher vorzugsweise experimentell oder numerisch (einen Überblick liefern [84,86]). Implikationen für die im Rahmen dieser Arbeit durchgeführten Schlag-/Stoßexperimente werden an gegebener Stelle im Kap. 3 erläutert.

Der kritische Wert des Spannungsintensitätsfaktors unter dynamischen Belastungsbedingungen, die dynamische Rißinitiierungszähigkeit $K_{I d}$, unterliegt generell ebenfalls dem Einfluß der Beanspruchungsrate. Ob dynamische Initiierungszähigkeiten im Vergleich zu statischen ansteigen oder abfallen ist stark materialabhängig. Wie aus Gl. (7) ersichtlich, hängt die Bruchzähigkeit wechselseitig mit der Festigkeit und der Größe der Prozeßzone zusammen. Ein Einfluß der Belastungsrate auf den Festigkeitswert bei vielen Materialien ist allgemein bekannt. Die Ausbildung der Prozeßzone kann, abhängig vom jeweiligen Deformationsmechanismus (und der Temperatur), ebenfalls durch die Dehnrate gesteuert sein. Infolge einer Steigerung der Beanspruchungsrate 
sinkt daher die Bruchzähigkeit, wenn versprödende Einflüsse überwiegen, und nimmt im Gegensatz dazu bei einer Zunahme der Duktilität höhere Werte an [84]. Hinweise auf experimentelle Untersuchungen der dynamischen Rißinitiierung an verschiedenen Materialien finden sich ebenfalls in [84].

\subsubsection{Dynamische Effekte bei instabiler Rißverlängerung}

Die Verlängerung eines Risses wird, wie in Abs. 2.2 beschrieben, dadurch angetrieben, daß das Energieangebot durch das Beanspruchungsfeld mindestens den Energiebedarf ( $\left.E_{\text {Bruch }}\right)$ deckt, der durch den Bruchwiderstand resultiert (s. Abb. 15). Ein sich darüber hinaus einstellendes Energieüberangebot $E_{\ddot{U}}$ beschleunigt die Rißufer zusätzlich und bestimmt somit den Geschwindigkeitsverlauf des Rißausbreitungsprozeßes. Das Energieüberangebot wird während der Rißausbreitung in kinetische Energie $E_{\text {kin }}$ umgewandelt, weshalb für laufende Risse die in Gl. (11) angegebene Bilanz des Energieflusses in die Prozeßzone um einen kinetischen Term zu ergänzen ist $[3,57,76]$ :

$$
G=\frac{\mathrm{d} U_{p}}{\mathrm{~d} a}-\frac{\mathrm{d} E_{\text {kin }}}{\mathrm{d} a} .
$$

Hierbei ergeben sich die überschüssige Energie $E_{\ddot{U}}$, wie in Abb. 15 für den Beginn der Rißausbreitung skizziert, und die kinetische Energie $E_{\text {kin }}$, die durch die Geschwindigkeit $v_{m}$ einzelner Massenpunkte aus dem relevanten Volumen $\mathrm{d} V$ (nahe dem Rißufer) bestimmt ist, für eine bestimmte kritische Spannung $\sigma_{c}$ aus:

$$
E_{\ddot{\mathrm{U}}}=\int_{a_{0}}^{a}(G-R) \mathrm{d} a=f\left(\sigma_{c}, a\right) \quad \text { und } \quad E_{\text {kin }}=\frac{1}{2} \varrho \int v_{m}^{2} \mathrm{~d} V=f\left(\sigma_{c}, a, v_{c}\right) .
$$

Die kinetische Energie ist, wie in Gl.(24) angegeben, aufgrund der bei der Beschleunigung der Rißufer wirkenden Trägheitskräfte von der Rißausbreitungsgeschwindigkeit

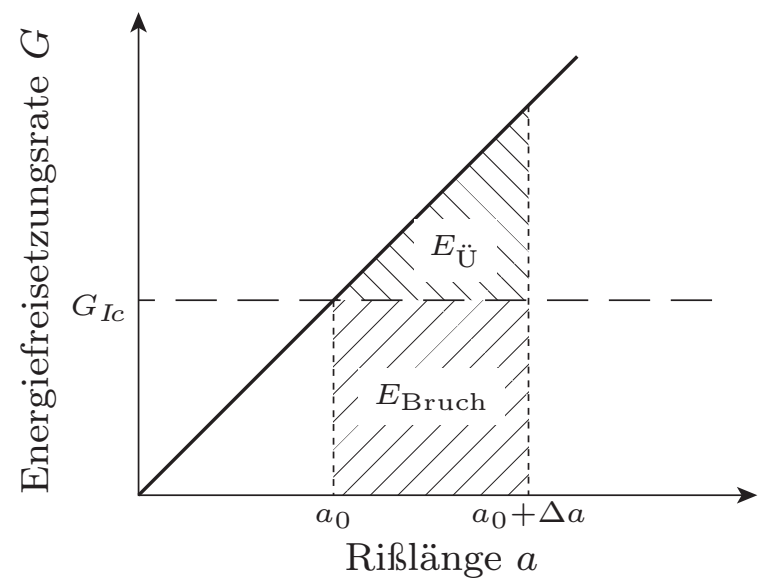

Abb. 15: Ausbildung eines Energieüberangebotes $E_{\ddot{\mathrm{U}}}$ bei laufenden Rissen. 
$v_{c}=\dot{a}$ abhängig. Auch die Ausbildung des Spannungs- und Verschiebungsfeldes an der Rißspitze eines sich ausbreitenden Risses ist daher dynamisch beeinflußt. Für einen sich mit der mit der Geschwindigkeit $v_{c}$ ausbreitenden Zugriß, bezüglich eines an der Rißspitze mitbewegten Koordinatensystemes $(\bar{x}=x-a(t)=\bar{r} \cos \bar{\varphi}$ und $\bar{y}=y=\bar{r} \sin \bar{\varphi}$ ), lautet entsprechend Gl. (4) die Definition des Spannungsintensitätsfaktors:

$$
K_{I}=K_{I}(t):=\lim _{\bar{r} \rightarrow 0} \sigma_{y}(\bar{r}, \bar{\varphi}=0, t) \sqrt{2 \pi \bar{r}} .
$$

Unter Verwendung von $\alpha_{i}=\sqrt{1-v_{c}^{2} / v_{i}^{2}}$ und $D=4 \alpha_{p} \alpha_{s}-\left(1+\alpha_{s}^{2}\right)^{2}$ sowie mit tan $\bar{\varphi}_{i}=$ $\alpha_{i} \tan \bar{\varphi}$, wobei $i=p, s$ und $\tan \bar{\varphi}=\bar{y} / \bar{x}$, ergeben sich für den singulären Teil des Rißspitzenspannungsfeldes eines laufenden Risses folgende Spannungskomponenten ([76], siehe auch $[45,49,87])$ :

$$
\begin{aligned}
\sigma_{\bar{x}} & =\frac{K_{I}}{\sqrt{2 \pi \bar{r}}} \frac{1+\alpha_{s}^{2}}{D}\left[\frac{\left(1+2 \alpha_{p}^{2}-\alpha_{s}^{2}\right) \cos \frac{\bar{\varphi}_{p}}{2}}{\sqrt{1-\left(\frac{v_{c} \sin \varphi}{v_{p}}\right)^{2}}}-\frac{4 \alpha_{p} \alpha_{s}}{1+\alpha_{s}^{2}} \frac{\cos \frac{\bar{\varphi}_{s}}{2}}{\sqrt{1-\left(\frac{v_{c} \sin \varphi}{v_{s}}\right)^{2}}}\right] \\
\sigma_{\bar{y}} & =\frac{K_{I}}{\sqrt{2 \pi \bar{r}}} \frac{1+\alpha_{s}^{2}}{D}\left[\frac{-\left(1+\alpha_{s}^{2}\right) \cos \frac{\bar{\varphi}_{p}}{2}}{\sqrt{1-\left(\frac{v_{c} \sin \varphi}{v_{p}}\right)^{2}}}+\frac{4 \alpha_{p} \alpha_{s}}{1+\alpha_{s}^{2}} \frac{\cos \frac{\bar{\varphi}_{s}}{2}}{\sqrt{1-\left(\frac{v_{c} \sin \varphi}{v_{s}}\right)^{2}}}\right] \\
\tau_{\bar{x} \bar{y}} & =\frac{K_{I}}{\sqrt{2 \pi \bar{r}}} \frac{2\left(1+\alpha_{s}^{2}\right) \alpha_{p}}{D}\left[\frac{\sin \frac{\bar{\varphi}_{p}}{2}}{\sqrt{1-\left(\frac{v_{c} \sin \varphi}{v_{p}}\right)^{2}}}-\frac{\sin \frac{\bar{\varphi}_{s}}{2}}{\sqrt{1-\left(\frac{v_{c} \sin \varphi}{v_{s}}\right)^{2}}}\right] .
\end{aligned}
$$

Für die Gültigkeit der vorstehenden Beziehungen wird vorausgesetzt, daß eventuelle Körperberandungen weit entfernt sind und somit die bei Rißfortschritt von den Rißufern in Form von Entlastungswellen abgestrahlte Energie nicht durch Reflexion zur Rißspitze zurückgelangt.

Das in Gl. (26) angegebene Spannungsfeld ist bis auf die Geschwindigkeitskorrekturterme mit dem Spannungsfeld nach Sneddon identisch (vergl. Gl. 1) und geht für $v_{c} \rightarrow 0$ in letzteres über. Allerdings steigen die Spannungen bei wachsenden Rißgeschwindigkeiten an und erreichen bei der Rayleighwellengeschwindigkeit unendlich hohe Werte, vorausgesetzt der Spannungsintensitätsfaktor bleibt endlich. Dabei steigt $\sigma_{\bar{x}}$ bei der Erhöhung der Rißgeschwindigkeit sehr viel schneller an als $\sigma_{\bar{y}}$. Dadurch reduziert sich, wie das Verhältnis der Spannungen auf dem Ligament zeigt [45]:

$$
\frac{\sigma_{\bar{y}}(\bar{\varphi}=0)}{\sigma_{\bar{x}}(\bar{\varphi}=0)}=\frac{4 \alpha_{p} \alpha_{s}-\left(1+\alpha_{s}^{2}\right)^{2}}{\left(1+2 \alpha_{p}^{2}-\alpha_{s}^{2}\right)\left(1+\alpha_{s}^{2}\right)-4 \alpha_{p} \alpha_{s}},
$$

die Mehrachsigkeit des Spannungsfeldes (Abb. 16). Diese Mehrachsigkeitsreduktion begünstigt die Ausbildung der Prozeßzone (vergl. Abs. 2.1.3) und hat einen erheblichen Einfluß auf den Bruchwiderstand (s. u.). 


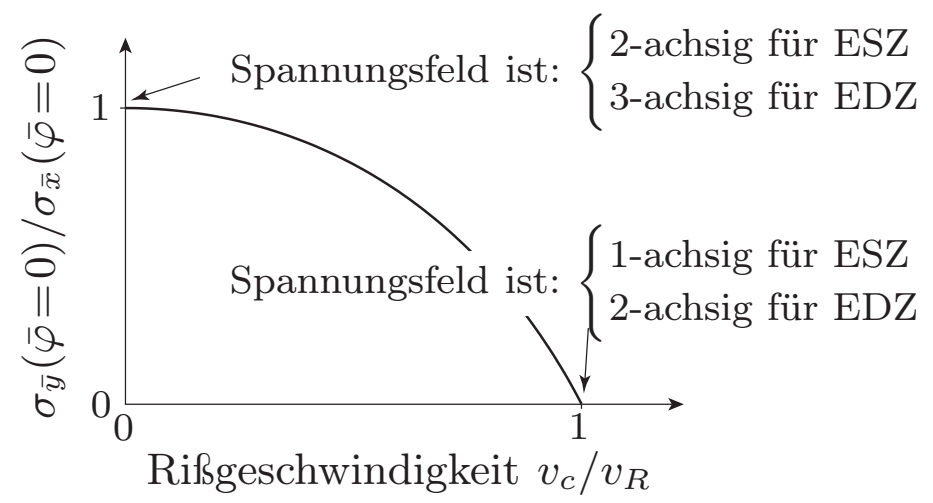

Abb. 16: Mehrachsigkeitsreduktion bei Rißbeschleunigung $(\nu=1 / 4)$.

Aus Gl. (26) ist ersichtlich, daß für die dynamische Betrachtungsweise die $1 / \sqrt{r}$ Singularität bestehen bleibt und das Rißspitzenspannungsfeld wie bei der statischen Betrachtungsweise durch den Spannungsintensitätsfaktor bestimmt wird. Daher ist, solange die Bedingung des Kleinbereichsfließens erfüllt ist, das bruchmechanische Konzept auf dynamische Fälle erweiterbar. Wobei für dynamische Rißausbreitung aufgrund des Dehnrateneffektes eher eine Verringerung der Prozeßzonengröße zu erwarten ist [88].

Der Aufbau von Rißspitzenspannungskonzentrationen ist ebenfalls von der Ausbreitungsgeschwindigkeit eines Risses abhängig. Dabei kann der dynamische Spannungsintensitätsfaktor $K\left(v_{c}>0\right.$ ) (im folgenden abkürzend mit $K\left(v_{c}\right)$ bezeichnet) mittels eines Korrekturfaktors, der sämtliche Geschwindigkeitseinflüsse beinhaltet, aus dem statischen Spannungsintensitätsfaktor $K\left(v_{c}=0\right)$ (kurz $\left.K(0)\right)$ bestimmt werden:

$$
K_{I}\left(v_{c}\right)=K_{I}(0) k\left(v_{c}\right) .
$$

Diese Beziehung gilt für alle praktisch relevanten Anwendungsfälle [76], solange eine Beeinflussung des originären Belastungszustandes ausgeschlossen werden kann; wobei die Entfernung möglicher Störquellen (Körperberandung, weitere Rißspitzen) und die Wellengeschwindigkeit zu berücksichtigen sind. Der geschwindigkeitsabhängige Korrekturfaktor $k\left(v_{c}\right)$ lautet näherungsweise [14]:

$$
k\left(v_{c}\right)=\frac{1-\frac{v_{c}}{v_{R}}}{\sqrt{1-v_{c} \frac{2}{v_{p}}\left(\frac{v_{s}}{v_{R}}\right)^{2}\left(1-\frac{v_{s}}{v_{p}}\right)^{2}}}
$$

und ist in Abb. 17a graphisch dargestellt. Der Wert des Korrekturfaktors $k$ (durchgezogene Linie) fällt vom Wert $k=1$ bei $v_{c}=0$ kontinuierlich auf den Wert $k=0$ bei $v_{c}=v_{R}$ ab. Demnach ist der Betrag des dynamischen Spannungsintensitätsfaktors stets geringer als der des statischen. Es wir deutlich, daß bei Rißausbreitungsvorgängen der Aufbau von Spannungsüberhöhungen bei steigenden Rißgeschwindigkeiten 


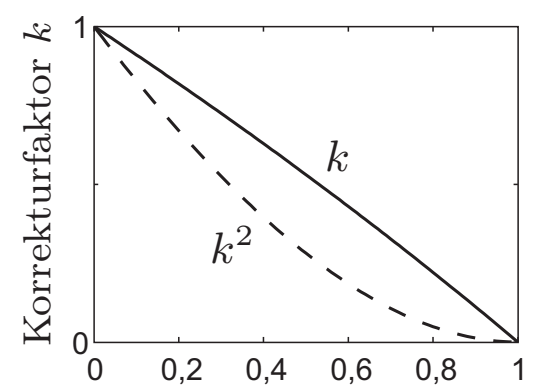
a)

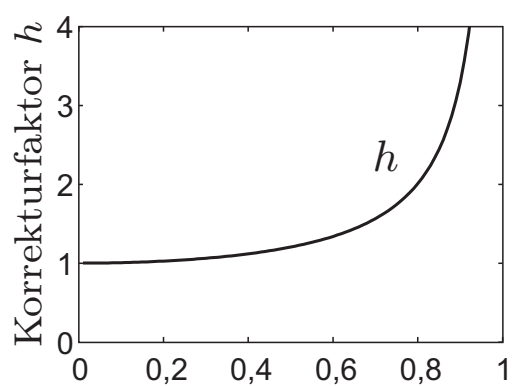

b)

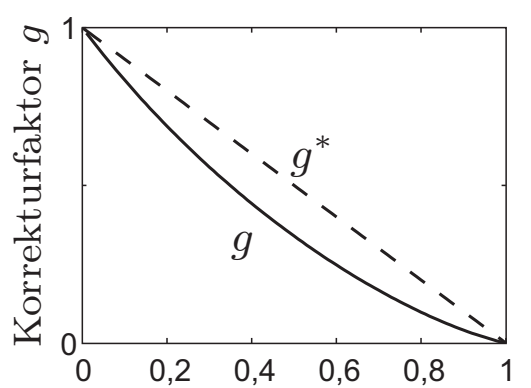

c)
Rißgeschwindigkeit $v_{c} / v_{R}$

Abb. 17: Korrekturfaktoren bei der Umrechnung von: a) $K_{I}(0)$ in $K_{I}\left(v_{c}\right)$, b) $K_{I}\left(v_{c}\right)$ in $G_{I}\left(v_{c}\right)$ und c) $K_{I}(0)$ oder $G_{I}(0)$ in $G\left(v_{c}\right)$ (für $\nu=1 / 4$ ).

zunehmend behindert wird. Gänzlich unterbunden wird der Aufbau von Spannungsüberhöhungen bei Erreichen der Rayleighwellengeschwindigkeit, die somit eine absolute Obergrenze für die Ausbreitungsgeschwindigkeit von Zugrissen markiert. Eine umfassendere Betrachtung (s.u.) zeigt, daß die Rayleighwellengeschwindigkeit nur eine theoretische Grenze darstellt; in der Praxis werden solch hohe Rißausbreitungsgeschwindigkeiten nie erreicht.

Auch die Energiefreisetzungsrate unterliegt unter dynamischen Bedingungen geschwindigkeitsabhängigen Einflüssen. Zur Bestimmung der dynamischen Energiefreisetzungsrate aus dem dynamischen Spannungsintensitätsfaktor (vergl. hierzu Gl. 15) ist ein weiterer geschwindigkeitsabhängiger Korrekturfaktor zu berücksichtigen [45,76]; für den ebenen Dehnungszustand gilt:

$$
G_{I}\left(v_{c}\right)=\frac{1-\nu^{2}}{E} K_{I}^{2}\left(v_{c}\right) h\left(v_{c}\right),
$$

mit:

$$
h\left(v_{c}\right)=\frac{v_{c}^{2} \alpha_{p}}{(1-\nu) v_{s}^{2}\left(4 \alpha_{p} \alpha_{s}-\left(1+\alpha_{s}^{2}\right)^{2}\right)} .
$$

Dabei steigt der Wert des Korrekturfaktors $h$ (Abb. 17b) von $h=1$ bei $v_{c}=0$ auf $h=\infty$ bei $v_{c}=v_{R}$ an. Im ebenen Spannungszustand entfällt der Faktor $1-\nu^{2}$ und die geänderten Steifigkeitsbedingungen sind über eine entsprechende Anpassung der Geschwindigkeiten zu berücksichtigen (s. Abs. 2.3.2, [76]). Somit stellt Gl. (30) die dynamische Erweiterung der Verknüpfung zwischen Spannungs- $K$ - und Energie- $G$ Bild (Gl. 15) dar, wobei die Geschwindigkeitseinflüsse bis in einen Bereich von etwa $60 \%-70 \%$ der Rayleighwellengeschwindigkeit gering $(<50 \%)$ sind.

Durch Einsetzen von Gl. (28) in Gl. (30) ergibt sich für die Beziehung zwischen der dynamischen Energiefreisetzungsrate und dem statischen Spannungsintensitätsfaktor 
insgesamt der Ausdruck:

$$
\begin{aligned}
G_{I}\left(v_{c}\right) & =\frac{1-\nu^{2}}{E} K_{I}^{2}(0) k^{2}\left(v_{c}\right) h\left(v_{c}\right) \\
& =G_{I}(0) g\left(v_{c}\right)
\end{aligned}
$$

Der Korrekturfaktor $g$ folgt aus der Kombination der geschwindigkeitsabhängigen Korrekturfaktoren $k$ und $h$ (die gegenläufige Monotonieeigenschaften aufweisen) zu:

$$
g\left(v_{c}\right)=k^{2}\left(v_{c}\right) h\left(v_{c}\right)
$$

und fällt, wegen der größeren Konvergenzgeschwindigkeit des Quadrates von $k$ (gestrichelte Kurve in Abb. 17a) gegenüber $h$, wie in Abb. 17c dargestellt, ebenfalls kontinuierlich vom Wert $g=1$ bei $v_{c}=0$ auf $g=0$ bei $v_{c}=v_{R}$ ab. Somit ergibt sich im Energie-G-Bild ein ähnlicher Verlauf wie im Spannungs- $K$-Bild. Vereinfachend kann der Korrekturfaktor $g$ hinreichend genau durch die lineare Näherung:

$$
g^{*}\left(v_{c}\right)=1-\frac{v_{c}}{v_{R}}
$$

(siehe gestrichelte Kurve in Abb. 17c, [1, 14,76]) ausgedrückt werden.

Die Kriterien für Rißinstabilität und Rißausbreitung gelten unter dynamischen Bedingungen analog denen der statischen Betrachtungsweise (vgl. Gln. 16 und 17), werden jedoch auf die dynamischen Beanspruchungsgrößen $K\left(v_{c}\right)$ oder $G\left(v_{c}\right)$ bezogen [89]. Zur Unterscheidung von den kritischen Werten des Spannungsintensitätsfaktors und der Energiefreisetzungsrate unter statischen Bedingungen wird im Spannungs- $K$-Bild die Rißlaufzähigkeit $K_{D}$ und im Energie- $G$-Bild die spezifische Bruchausbreitungsenergie $G_{D}$ als dynamische Bruchwiderstandsgrößen eingeführt. Dabei ist zu beachten, daß diese materialspezifischen kritischen Werte von Spannungsintensitätsfaktor und Energiefreisetzungsrate generell keine einparametrige Beschreibung des Bruchprozesses ermöglichen, sondern vielmehr, genau wie die Beanspruchungsparameter, von der Rißausbreitungsgeschwindigkeit abhängen:

$$
K_{D}=K_{D}\left(v_{c}\right) \quad \text { und } \quad G_{D}=G_{D}\left(v_{c}\right)
$$

wobei für übliche Rißausbreitungsgeschwindigkeiten der Korrekturfaktor (s. Gl. 30a) vernachlässigbar ist, so daß beide Größen, zumindest für qualitative Aussagen, weiterhin äquivalent verwendet werden können.

Für die Bruchwiderstand-Bruchgeschwindigkeits-Beziehung eines laufenden Risses ergibt sich prinzipiell der in Abb. 18 (für Modus-I) dargestellte Verlauf. Dieser Verlauf wird durch das Zusammenwirken teilweise gegenläufiger Einflüsse bestimmt: Einerseits dominieren bei geringen Bruchgeschwindigkeiten aufgrund steigender lokaler Beanspruchungsraten materialversprödende Effekte, die zu einer Verringerung der Prozeßzonengröße und somit der Energiedissipation führen und ein Absinken des 


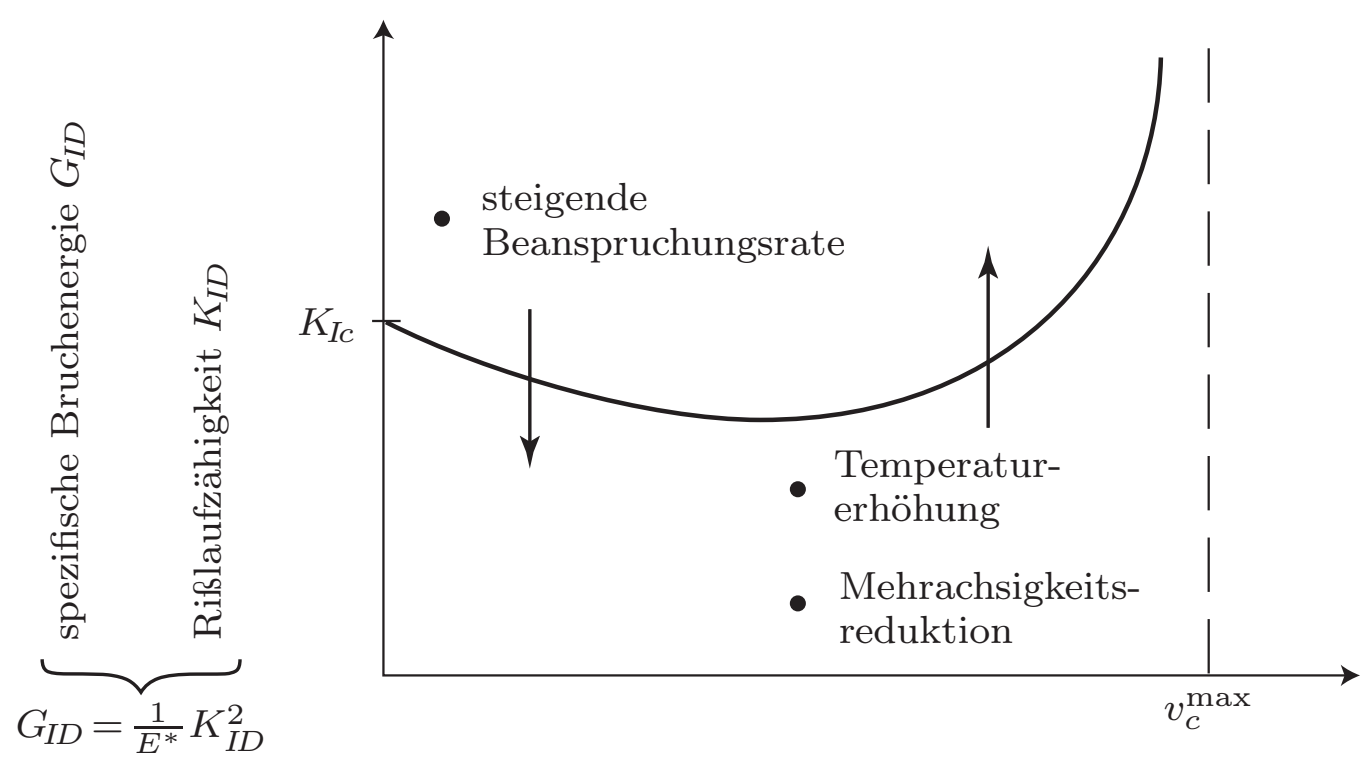

Rißgeschwindigkeit $v_{c}$

Abb. 18: Prinzip des Verhältnisses zwischen Bruchzähigkeit (Bruchenergie) und der Ausbreitungsgeschwindigkeit laufender Risse, schematisch.

Bruchwiderstandes bewirken. Andererseits resultiert die Reduktion der Mehrachsigkeit des Spannungsfeldes, genauso wie die Erhöhung der Temperatur an der Rißspitze, in einer Zunahme der Prozeßzonengröße und verursacht einen steilen Anstieg des Bruchwiderstandes bei hohen Bruchgeschwindigkeiten. Da die Erhöhung des Bruchwiderstandes, zusätzlich zu den bereits angeführten Effekten, einer Rißbeschleunigung entgegenwirkt, ist die sich einstellende maximale Rißausbreitungsgeschwindigkeit $v_{c}^{\max }$ deutlich kleiner als die theoretisch mögliche, die, wie vorstehend beschrieben, durch die Rayleighwellengeschwindigkeit gegeben ist. Experimentelle Untersuchungen zeigen, daß höchstens etwa $70 \%$ der Rayleighwellengeschwindigkeit erreicht werden [5, 90-92].

Vorhersagen zur Bruchwiderstand-Bruchgeschwindigkeits-Kurve eines bestimmten Materials sind aufgrund der Komplexität der vorstehend genannten Einflüsse und ihres Zusammenwirkens im allgemeinen nicht möglich. Allerdings zeigt der Vergleich von Ergebnissen experimenteller Untersuchungen an einer Vielzahl von Materialien, daß ein Absinken der Bruchzähigkeit im Bereich niedriger Bruchgeschwindigkeiten bei Materialien, die von vornherein sprödes Materialverhalten aufweisen, nicht zu erwarten ist. Als Beispiel hierzu ist in Abb. 19 eine Meßkurve für das Epoxidharz Araldit B angegeben. Zugleich ist in der Abbildung zu erkennen, daß die Bruchhöchstgeschwindigkeit für dieses Material etwa $400 \mathrm{~m} / \mathrm{s}$ beträgt (zum Vergleich: $\left.v_{R} \approx 1000 \mathrm{~m} / \mathrm{s}\right)$. Dazu ist zu bemerken, daß es keineswegs möglich ist, die Bruchhöchstgeschwindigkeit aus den elastischen Parametern eines Materials abzuleiten, obwohl es üblich ist die materialspezifische maximale Bruchgeschwindigkeit durch ihr Verhältnis zu den Wellengeschwindigkeiten zu beschreiben. Vielmehr sind selbst 


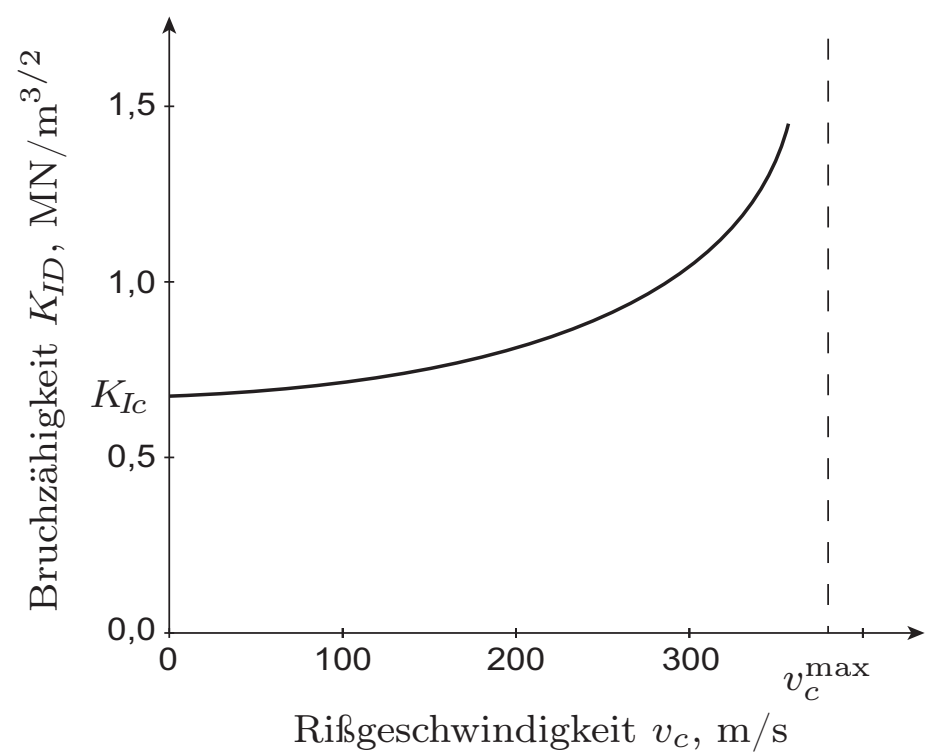

Abb. 19: Meßbeispiel zur Bruchwiderstand-Bruchgeschwindigkeits-Beziehung für das spröde Material Araldit B (aus [93]).

für Materialien derselben Materialklasse, mit ähnlichen elastischen Parametern, deutliche Unterschiede beim Vergleich der Bruchhöchstgeschwindigkeiten zu beobachten. Als Erklärung hierfür werden häufig mikrostrukturelle Ursachen angeführt.

Eine weitere Beobachtung zeigt, daß der steigende Energieumsatz bei steigenden Rißausbreitungsgeschwindigkeiten durch eine kontinuierliche Erhöhung der Rauhigkeit der neu geschaffenen Bruchfläche abgebildet wird [94]. Dieser Prozeß gipfelt darin, daß der Riß bei Erreichen der maximalen Bruchgeschwindigkeit gabelt und die Rißausbreitung danach auf mehreren Rißästen gleichzeitig erfolgt [95].

Obwohl nicht Gegenstand der vorliegenden Arbeit sei erwähnt, daß die Arretierung von Rissen ebenfalls dynamischen Gesetzmäßigkeiten unterliegt [45, 96, 97]. Unter welchen Bedingungen Rißausbreitung gestopt wird, wird durch die Rißarrestzähigkeit („Crack Arrest Toughness“) bestimmt. Sie stellt wie die (statische) Rißinitiierungszähigkeit eine Materialkonstante dar, weist aber nicht denselben Betrag auf. Die experimentelle Untersuchung des Rißarrestphänomens hat in vielerlei Hinsicht das tiefere Verständnis dynamischer bruchmechanischer Prinzipien geprägt. 


\subsection{Anwendung der Bruchmechanik auf geologische Materialien}

Das vielfältige Auftreten von Bruchprozessen in der Erde hat dazu geführt, daß die Bruchmechanik, ursprünglich entwickelt auf der Grundlage ingenieurmäßigen Fragestellungen, auch in den Geowissenschaften eingesetzt wird [98]. Im Gegensatz zur Anwendung der Bruchmechanik bei Bemessungen zur Planung und Optimierung von Strukturen ist es hier in der Regel nicht möglich die Eigenschaften der betreffenden Materialien auszuwählen oder zu verändern. Das mechanische Verhalten natürlicher geologischer Materialien wird wesentlich durch die bei ihrer Genese oder während der thermo-mechanischen Beanspruchungsgeschichte entstandenen Fehlstellen bestimmt. Im Rahmen einer bruchmechanischen Betrachtung sind hierbei im Gestein enthaltene Mikrorisse oder -poren sowie auf der Makroebene Störungen und Klüfte von Bedeutung. Diese präexistierenden Risse sind in der Erde vorwiegend Druckspannungen ausgesetzt. Unabhängig vom Beanspruchungsmodus erfolgt die Ausbreitung in der Regel dennoch als Zug(Modus-I)-Riß (Abb. 20a, [99], vergl. Abs. 4.2). Die Rißausbreitung erfolgt hierbei aufgrund von besonderen Materialeigenschaften, im Gegensatz zu herkömmlichen Strukturmaterialien mit spröder Rißausbreitung, langsam und subkritisch oder schnell und überkritisch (vergl. Abs. 4.2). Daneben resultieren aus den in-situ Umgebungsbedingungen vielfältige Einflußfaktoren, die bei der Rißinitiierung und -ausbreitung in Gestein zu berücksichtigen sind, z. B. Effekte der Temperatur, der Anwesenheit von Formationsfluiden, des Druckes (einschließlich des Fluiddruckes) und der Zeit. Zusätzlich können zudem Materialheterogenitäten auf verschiedenen Längenskalen die Bruchzähigkeit beeinflussen. Aufgrund dessen konzentrieren sich Untersuchungen zur Bestimmung eines intrinsischen bruchmechanischen Parameters in der Gesteinsmechanik (im Gegensatz dazu zeigen Fe-

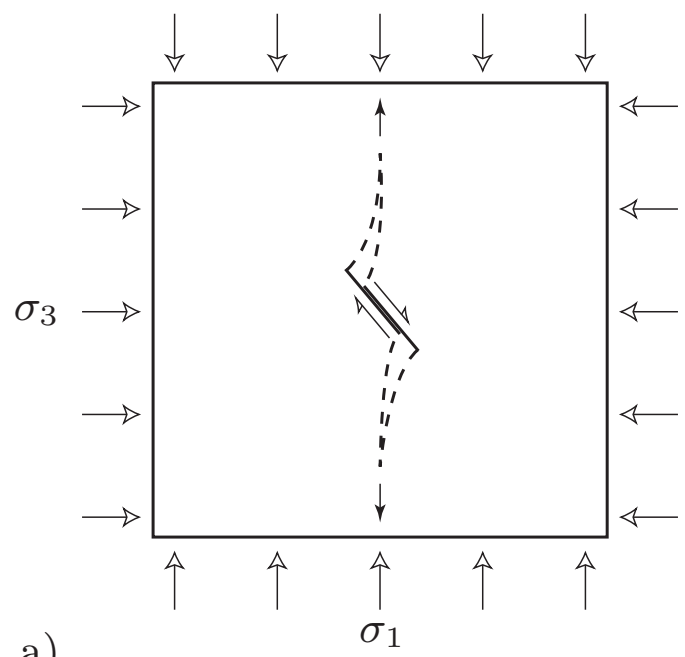

a)

Abb. 20: Rißausbreitung unter Druckbeanspruchung: a) Zugrißausbreitung, b) Scherrißausbreitung (nach [3]).

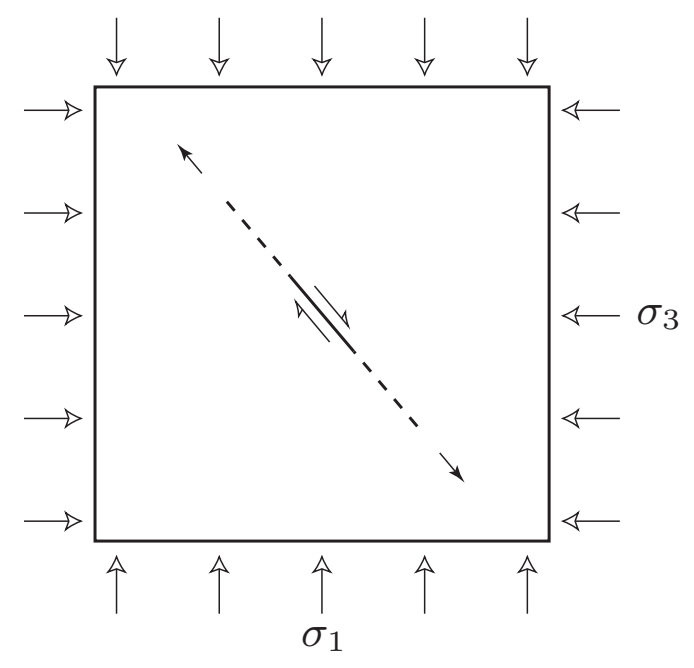

b) 
stigkeitsbetrachtungen eine Abhängigkeit von der Größe der betrachteten Struktur) hauptsächlich auf die Zug(Modus-I)-Rißinitiierung. Experimente zur Bestimmung der Abhängigkeit der Bruchzähigkeit von der Rißgeschwindigkeit wurden lediglich in Ausnahmefällen und im subkritischen Bereich durchgeführt [100,101]. Dynamische Rißausbreitung in Gesteinen bis in den Bereich der maximalen Rißgeschwindigkeit wurde ohne Berücksichtigung der Rißlaufzähigkeit untersucht [102-104].

Frühe Untersuchungen an Gesteinen haben gezeigt, daß die Rißinitiierungszähigkeit $K_{I c}$ bei Messungen an Gesteinen Abhängigkeiten von der Probengeometrie aufweist. Hier ist vor allem eine Abhängigkeit von der Rißlänge zu nennen [105, 106]. Als Resultat einer in Gesteinen oftmals sehr ausgedehnten Prozeßzone* [107] ist häufig die Probengröße zu gering um gültige Bruchzähigkeiten zu bestimmen [30,108]. Als Konsequenz daraus wurde das Testverfahren zur Bestimmung von reproduzierbaren Bruchzähigkeiten von der Internationalen Gesellschaft für Gesteinsmechanik (ISRM) standardisiert $[109,110]$. Diese Prüfvorschriften basieren hauptsächlich auf Arbeiten mit dem Ziel der Erweiterung des Konzeptes der kritischen $K$-Faktoren auf Proben, die nicht den Bedingungen der linear-elastischen Bruchmechanik entsprechen und von den Bestimmungen der ASTM-Prüfvorschrift (s. Abs. 2.1.4) abweichen. Diese wurden derart angepaßt, daß die Verwendung von Gesteinsproben möglich ist, die mit wenig Aufwand aus dem häufig in Form von Bohrkernen vorliegenden Ausgangsmaterial gewonnenen werden. Als Versuchsbedingung ist eine langsame Durchführung der Experimente mit subkritischer Rißausbreitung und wiederholten EntlastungsWiederbelastungs-Schleifen (Hyseteresen) vorgeschrieben, deren Realisierung durch die Verwendung von V-förmigen Starterkerben (Chevron-notches) begünstigt wird. Diese Art der Starterkerbe sorgt zudem dafür, daß trotz der mittels Sägeschnitten eingebrachten Initiierungsstelle die Bruchzähigkeit im Verlauf des Experimentes von einer scharfen Rißspitze bestimmt wird [111]. Die ISRM-Vorschriften enthalten eine zweistufige Korrektur der in den Experimenten gemessenen scheinbaren Bruchzähigkeit. Die erste Korrektur dient der Elimination von Verfälschungen, die von der sogenannten Nichtlinearität der Ergebnisse herrühren und aus der Steigung der Hysteresen bestimmt wird (unter Vermeidung des Begriffes „Plastizitätskorrektur“, der für Gesteine nicht adäquat erscheint - vergl. Abs. 2.1.3). Die zweite Korrektur berücksichtigt einen eventuellen Versatz zwischen der im Experiment gemessenen maximalen Last und der kritischen Last an der Rißposition, die der größten Probensteifigkeit entspricht. Während die erste Korrektur infolge des ,strain softening“-Verhaltens (entspricht ,slip weakening“ unter Scherbedingungen) von Gesteinen zu einer Erhöhung gegenüber den scheinbaren Bruchzähigkeitswerten führt werden diese in der zweiten Stufe reduziert. Eine Auswertung in Bezug auf die für gültige Experimente notwendigen Probengrößen ist enthalten in [112].

*Der Prozeßzonenradius wird wegen der polykristallinen Mikrostruktur von Gesteinen häufig, ähnlich dem Vorgehen bei Keramiken und abweichend von der Definition über die Rißspitzenspannung (s. Abs. 2.1.3), mit der Korngröße in Zusammenhang gebracht. Abschätzungen liefern einen Prozeßzonenradius entsprechend dem zehnfachen des Korndurchmessers, verschiedentlich sogar darüber [98, 101]. 
Im Gegensatz zu der im Ingenieurwesen und für die Geotechnik gebräuchlichen konservativen Betrachtungsweise werden für Sicherheitsabwägungen im Hinblick auf Bruchvorgänge bei Erdbeben $[113,114]$ Versagenswahrscheinlichkeiten benötigt. Hierfür sind bezüglich der Initiierungszähigkeiten aufgrund der zufälligen Orientierung präexistierender Fehlstellen im herrschenden Spannungsfeld sämtliche Beanspruchungsmoden zu berücksichtigen (siehe Abschnitt 2.2.3). Labormessungen von Scherbruchzähigkeiten an Gesteinen sind selten, ein Standard existiert nicht [115].

Auch bei der Rißausbreitung können sich Scherbedingungen einstellen. [116]. Inhomogenitäten und/oder Einflüsse aus den Spannungsbedingungen führen dazu, daß die Rißausbreitung selbstähnlich in Richtung des Startrisses erfolgt (Abb. 20b, [3, 117]). Die Rißausbreitung im ersten Fall resultiert dabei aus der Verbindung einer Vielzahl von Mikrozugrissen zu einem Makroriß [33,118,119], der zweite Fall entspricht der Ausbreitung eines einzelnen Scherrisses bei hohen Manteldrücken, für die die Zugrißausbreitung unterdrückt wird. Eine solche Scherbruchausbreitung ist in der Regel an Schwächezonen gebunden. Aufgrund des oftmals langsamen Spannungsaufbaus kann die Rißlänge bzw. die Länge der Schwächezone die entscheidende Initiierungsgröße sein [120,121], der Anfangsbereich der Scherrißausbreitung wird somit ebenfalls durch subkritische Rißausbreitung dominiert.

Im Bezug auf die Energiebilanz sind bei der Scherbruchausbreitung neben der dissipierten Bruchenergie und der in Form seismischer Wellen abgestrahlten kinetischen Energie als weitere wesentliche Komponente Reibungsphänomene zu berücksichtigen. Die Reibungsenergie kann beträchtliche Größen annehmen, sie führt u. a. zu lokaler Aufschmelzung und der Bildung amorpher Gesteinsgläser, sogenannter Pseudotachylite [122,123]. Die Abschätzung der Energiedissipation bei Erdbeben in einer geschlossenen Betrachtung ist nicht möglich, da hierfür entscheidende Einflußgrößen unbekannt sind (z. B. [124]). Alternativ wird ein einfaches Modell benutzt, um den Anteil der Bruchenergie bei Erdbeben näherungsweise anzugeben:

$$
G_{c}=\Delta \sigma \delta .
$$

In dem Modell ist der Spannungsabfall $\Delta \sigma=\sigma_{i}-\sigma_{r}$ (stress drop) während eines Bruchereignisses, von einem initialen Wert $\sigma_{i}$ auf das Niveau der residualen Reibung $\sigma_{r}$, verknüpft mit der Rißverlängerung $\delta$ (slip-distance) eines idealisiert als eben angenommenen Risses. Die Aussagekraft einer solchen Näherungslösung ist allerdings begrenzt, insbesondere bleibt die tatsächliche Variation des Spannungsfeldes unberücksichtigt. Detailliertere Analysen, die Heterogenitäten im Bezug auf z. B. die tatsächliche Spannungsverteilung, ungleichmäßige ,slip“-Verteilung oder die Bruchgeschwindigkeit berücksichtigen, sind an die Verwendung weiterer, schwierig zu erlangender Eingangsparameter geknüpft. Deren Gewinnung aus der Erdbebenbeobachtung ist dabei an die Erdoberfläche gebunden, wie etwa bei Untersuchungen des Verformungsfeldes mittels geodätischer Daten oder lokal begrenzt auf Bohrlochdaten. Die häufigsten Informationsquellen ist die indirekte Analyse seismischer Wellen 
oder die paläoseismische Untersuchung von Störungen bzw. Klüften. Eine umfassende Beschreibung von Vorgängen bei Erdbeben ist mit der zur Verfügung stehenden Datenbasis zum heutigen Zeitpunkt nicht möglich, zumal unter Berücksichtigung hoher Bruchgeschwindigkeiten in der Größenordnung der Ausbreitungsgeschwindigkeit seismischer Wellen [125-128], wie sie in Feldbeispielen beobachtet wird [129,130].

Ähnliche Schwierigkeiten treten bei dem Versuch auf, Ergebnisse zur Energiedissipation vom Labor mit in-situ-Gegebenheiten in Übereinstimmung zu bringen $[124,131]$. Bruchenergiemessungen in Laborexperimenten variieren von ca. $G_{c}=$ $1 \mathrm{~J} / \mathrm{m}^{2}$ für Einkristalle unter Zug(Modus-I)-Initiierungsbedingungen bis ca. $G_{c}=$ $10^{4} \mathrm{~J} / \mathrm{m}^{2}$ in Experimenten an polykristallinen Gesteinen unter triaxialen Beanspruchungsbedingungen. Experimente an polykristallinen Gesteinen unter Zug(ModusI)-Initiierungsbedingungen zeigen ebenfalls eine steigende Tendenz der Bruchzähigkeit bei Anliegen eines Manteldruckes. Wohingegen sich die Bruchzähigkeit bei der Anwesenheit korrosiver Fluide und unter langsamer Beanspruchung reduziert. Die Labordaten decken sich nicht mit Ergebnissen für Erdbeben. Hier werden, mit unterschiedlichen Ansätzen, relativ einhellig Energien im Bereich von $G_{c}=10^{6} \mathrm{~J} / \mathrm{m}^{2}$ bis $G_{c}=10^{7} \mathrm{~J} / \mathrm{m}^{2}$ abgeschätzt, beeinflußt allerdings durch die o. a. Unzulänglichkeiten der verwendeten Modellvorstellungen. Die Unsicherheit der Abschätzungen zeigt ein Beispiel, bei dem für dasselbe Erdbeben von verschiedenen Autoren Werte von $G_{c}=10^{2} \mathrm{~J} / \mathrm{m}^{2}$ bzw. $G_{c}=10^{7} \mathrm{~J} / \mathrm{m}^{2}$ angegeben werden. Ein Zusammenhang mit der Bruchausbreitungsgeschwindigkeit wird durch die relativ niedrige, als sehr verläßlich klassifizierte Bruchenergie von $G_{c}=10^{2} \mathrm{~J} / \mathrm{m}^{2}$ eines langsamen Bruchereignisses (creep event) angezeigt [114,131]. Um das Verständnis tektonischer Bruchprozesse zu steigern sind daher neue Konzepte und entsprechende Laborergebnisse nötig, die die Berücksichtigung unterschiedlicher Rißausbreitungsgeschwindigkeiten einschließen [132, 133]. 


\section{Methodik}

\section{$3.1 \quad$ Vorbemerkungen}

Die experimentelle Untersuchung von Bruchlaufenergien in Gestein erfordert den Einsatz verfeinerter Methoden, die sowohl bei der Erzeugung als auch bei der Beobachtung von Rißausbreitungsprozessen stark von den üblichen bruchmechanischen Standardverfahren abweichen: Zum einen sind zur Untersuchung der Bruchgeschwindigkeits-Bruchenergie-Beziehung für den gesamten Geschwindigkeitsbereich - von der Initiierung bis zum Erreichen der maximalen Bruchgeschwindigkeit - spezielle Belastungsungstechniken einzusetzen, die das für den angestrebten Geschwindigkeitsbereich adäquate Energieangebot zur Verfügung stellen. Zum anderen sind die Bruchlaufenergien im Verlauf des Ausbreitungsprozesses zu bestimmen und können daher generell nicht aus den globalen Beanspruchungsparametern abgeleitet werden. Sie werden folglich aus dem lokalen Beanspruchungsfeld direkt an der Rißspitze ermittelt. Da die so ermittelten Energiebeträge zusätzlich der momentanen Rißgeschwindigkeit zuzuordnen sind, empfiehlt es sich ein Verfahren einzusetzen, bei dem die Bruchlaufenergie und die Rißgeschwindigkeit gleichzeitig bestimmt werden. $\mathrm{Zu}-$ sätzlich muß das Verfahren bis in den Bereich sehr hoher Geschwindigkeiten anwendbar sein (siehe z. B. [134, 135]). Als in diesem Sinne für eine quantitative Untersuchung geeignet bieten sich grundsätzlich verschiedene optische Methoden an, insbesondere das schattenoptische Kaustikenverfahren (optisches Linienverfahren, [87], s. Anhang A) sowie das photoelastische Verfahren der Spannungsoptik (optisches Flächenverfahren, [22], s. Anhang A). Diesen Verfahren wurde jedoch ein von Dally und Sanford $[136,137]$ eingeführtes, optimiertes Dehnungsmeßstreifen-Ketten-Verfahren (punktuelles Verfahren) vorgezogen, das sich für die vorliegende Untersuchung als besonders geeignet erwies. Dieses wird im folgenden vorgestellt (Abs. 3.2). Auf die experimentelle Realisierung unterschiedlicher Energieniveaus bei Rißinitiierung und -ausbreitung wird im zweiten Teil dieses Kapitels eingegangen Abs. 3.3.

\subsection{Bestimmung von Rißlaufbruchzähigkeiten mit dem opti- mierten Dehnungsmeßstreifen-Ketten-Verfahren}

\subsubsection{Prinzip des optimierten Dehnungsmeßstreifenverfahrens bei stationären Rissen}

Bevor auf den Einsatz der Methode bei laufenden Rissen eingegangen wird (siehe Abs. 3.2.2), werden die Prinzipien des Dehnungsmeßstreifenverfahrens am Beispiel 
stationärer Risse erläutert. Bei dem Dehnungsmeßstreifenverfahren werden in der Nähe der Rißspitze Dehnungsmeßstreifen (DMS) appliziert. Dabei sind grundsätzlich weder der Abstand $r$ des Dehnungsmeßstreifens zur Rißspitze, noch der Winkel $\varphi$ zum Ligament oder die Aus-/Meßrichtung $\alpha$ (vergl. Abb. 21a) vorgegeben.

Wesentlich ist bei der Festlegung dieser Größen, daß der Meßabstand zur Bestimmung von Spannungsintensitätsfaktoren möglichst gering zu halten ist $\left(r_{\mathrm{opt}}=r \rightarrow \min \right)$, allerdings sind hierbei gewisse Einschränkungen zu berücksichtigen. Gemäß den in Kapitel 2 vorgestellten Grundsätzen des $K$-Konzeptes ist für eine einparametrige bruchmechanische Charakterisierung mittels Spannungsintensitätsfaktoren die Applikation begrenzt auf den Bereich, in dem das Spannungsfeld mit hinreichender Genauigkeit durch das singuläre erste Glied der Spannungsverteilung charakterisiert wird. In diesem Bereich können die Einflüsse der als Störterme auftretenden höheren Glieder der Spannungsverteilung sowie der Prozeßzone vernachlässigt werden (s. Abb.21b). Da jedoch in der Praxis Störtermeinflüsse nicht kategorisch auszuschließen sind müssen diese angemessen berücksichtigt werden. Dies wird anhand der Reihenentwicklung des Rißspitzenspannungs-/dehnungsfeldes beispielhaft für Zug(ModusI)-Beanspruchungsbedingungen erläutert; gemäß Gl. (5) gilt für das Rißspitzenspannungsfeld:

$$
\sigma_{i j}=\frac{K}{\sqrt{2 \pi r}} f_{i j}(\varphi)+B_{0} r^{0} g_{i j}(\varphi)+A_{1} r^{\frac{1}{2}} h_{i j}(\varphi)+B_{1} r^{1} i_{i j}(\varphi)+\cdots
$$

Folglich ist für den ebenen Spannungszustand (wie er an einer Probenoberfläche vorliegt, s. Abschnitt 2.1.4 - S. 11) das Dehnungsfeld für einen Zug(Modus-I)-Riß
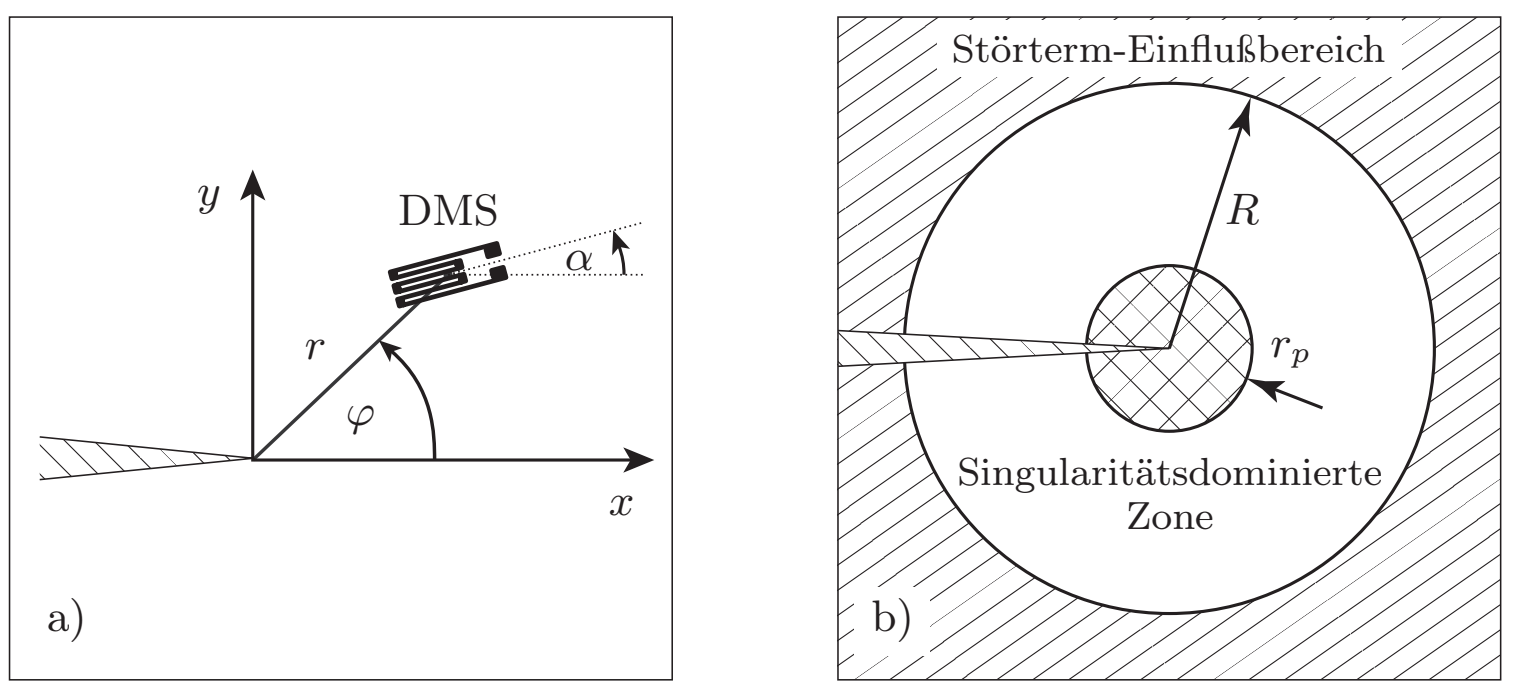

Abb. 21: DMS-Applikationsprinzip: a) Applikationsparameter, b) Parametrisierung des Spannungsfeldes (der innere, kreuzschraffierte Bereich gibt die Prozeßzone wieder). 
gegeben durch [136]:

$$
\begin{aligned}
\epsilon_{x}^{I}=\frac{1}{E}[ & \frac{K_{I}}{\sqrt{2 \pi r}} \cos \frac{\varphi}{2}\left(\frac{1-\nu}{1+\nu}-\sin \frac{\varphi}{2} \sin \frac{3 \varphi}{2}\right)+2 B_{0} r^{0} \\
& \left.+A_{1} r^{\frac{1}{2}} \cos \frac{\varphi}{2}\left(\frac{1-\nu}{1+\nu}+\sin ^{2} \frac{\varphi}{2}\right)+2 B_{1} r^{1} \cos \varphi+\cdots\right] \\
\epsilon_{y}^{I}=\frac{1}{E}[ & {\left[\frac{K_{I}}{\sqrt{2 \pi r}} \cos \frac{\varphi}{2}\left(\frac{1-\nu}{1+\nu}+\sin \frac{\varphi}{2} \sin \frac{3 \varphi}{2}\right)-2 \nu B_{0} r^{0}\right.} \\
& \left.+A_{1} r^{\frac{1}{2}} \cos \frac{\varphi}{2}\left(\frac{1-\nu}{1+\nu}-\sin ^{2} \frac{\varphi}{2}\right)-2 \nu B_{1} r^{1} \cos \varphi+\cdots\right] \\
\gamma_{x y}^{I}=\frac{2(1+\nu)}{E} & {\left[\frac{K_{I}}{2 \sqrt{2 \pi r}} \sin \varphi \cos \frac{3 \varphi}{2}-\frac{A_{1}}{2} r^{\frac{1}{2}} \sin \varphi \cos \frac{\varphi}{2}-2 B_{1} r^{1} \sin \varphi+\cdots\right] . }
\end{aligned}
$$

Wird durch die Ausrichtung der Dehnungsmeßstreifen - d.h. durch Vorgabe der Meßrichtung - der Winkel $\alpha$ als zusätzliche Einflußgröße eingeführt, ergibt sich für die Dehnung $\epsilon_{\alpha}$ nach der Rotation der Koordinaten auf das Orthogonalsystem bzgl. der Meßrichtung nach Dally und Sanford [136]:

$$
\begin{aligned}
\epsilon_{\alpha}^{I}=\frac{1}{E} & \left\{\frac{K_{I}}{\sqrt{2 \pi r}}\left[\frac{1-\nu}{1+\nu} \cos \frac{\varphi}{2}+\frac{1}{2} \sin \varphi\left(\cos \frac{3 \varphi}{2} \sin 2 \alpha-\sin \frac{3 \varphi}{2} \cos 2 \alpha\right)\right]\right. \\
& +B_{0} r^{0}[1-\nu+(1+\nu) \cos 2 \alpha] \\
& +A_{1} r^{\frac{1}{2}}\left[\frac{1-\nu}{1+\nu} \cos \frac{\varphi}{2}+\frac{1}{2} \sin \varphi\left(\sin \frac{\varphi}{2} \cos 2 \alpha-\cos \frac{\varphi}{2} \sin 2 \alpha\right)\right] \\
& +B_{1} r^{1}[(1-\nu) \cos \varphi+(1+\nu)(\cos \varphi \cos 2 \alpha-2 \sin \varphi \sin 2 \alpha)] \\
& +\cdots
\end{aligned}
$$

Die Anzahl der eingesetzten Dehnungsmeßstreifen legt über die Länge der Reihe (maximale Anzahl der betrachteten Reihenglieder, ohne daß das Problem unterbestimmt ist) fest, wie exakt der Spannungsintensitätsfaktor bestimmt wird - prinzipiell gilt hierbei: pro Meßstelle ergibt sich ein Reihenglied, sofern für die Dehnungsmeßstreifen identische Werte für $\alpha$ und $\varphi$ eingestellt werden. Demnach wird der Spannungsintensitätsfaktor mit einem Dehnungsmeßstreifen aus dem ersten Reihenglied bestimmt, durch Hinzunahme eines zweiten Dehnungsmeßstreifens ließe sich die Genauigkeit um eine Ordnung steigern und so fort. Aufgrund der Steigerung des Aufwandes, da die Materialkosten in nicht unerheblichem Umfang zunehmen sowie wegen des bei 
laufenden Rissen begrenzten Nutzens (siehe Anhang B) wurde dieses Verfahren zur Genauigkeitssteigerung nicht eingesetzt. Bürgel nutzte eine solche Extrapolationsmethode mit mehreren Dehnungsmeßstreifen bei stationären Rißspitzen in dynamischen Scher(Modus-II)-Experimenten im Rahmen eines Kalibrationsverfahrens [138].

Kostenneutral wird in der vorliegenden Arbeit die Genauigkeit dadurch erhöht, daß Besonderheiten der Spannungsverteilung ausgenutzt werden. Betrachtet man die vorstehende Gleichung, stellt man fest, daß der $B_{0}$-Term, im Gegensatz zu den übrigen Reihengliedern, nur die Meßrichtung als freien Parameter aufweist:

$$
\epsilon_{\alpha}^{I}=\frac{1}{E}\left(\frac{K_{I}}{\sqrt{2 \pi r}} f(\varphi, \alpha, \nu)+B_{0} r^{0} g(\alpha, \nu)+A_{1} r^{\frac{1}{2}} h(\varphi, \alpha, \nu)+\cdots\right) .
$$

Wie zuerst von Dally und Sanford [136] gezeigt, wird dieser Term (unabhängig vom Betrag des Koeffizienten $B_{0}$ ) für beliebige $r$ und $\varphi$ in der Meßrichtung $\alpha=\alpha_{\mathrm{opt}}(\nu)$ eliminiert:

$$
\alpha_{\mathrm{opt}}=\frac{1}{2} \arccos \frac{\nu-1}{1+\nu} .
$$

Darüber hinaus wird die Genauigkeit weiter erhöht, indem eine bezüglich der Variation des Dehnungsfeldes mit $\varphi$ ausgezeichnete Meßstelle verwendet wird. Bei Applikation eines Dehnungsmeßstreifens mit fester Meßrichtung $\alpha_{\mathrm{opt}}$ hat das dritte Reihenglied, der $A_{1}$-Term, nach Dally und Sanford [136] eine Nullstelle für $\varphi=\varphi_{\mathrm{opt}}$ :

$$
\varphi_{\mathrm{opt}}=2 \arctan \left(-\cot 2 \alpha_{\mathrm{opt}}\right) .
$$

Die Bedeutung dieses Zusammenhangs verdeutlicht eine Skizze der Winkelabhängigkeit der Reihenglieder des Dehnungsfeldes (Abb. 22, vergl. Gl. 38). An der Nullstelle verschwindet zusätzlich zu dem Störeinfluß des konstanten Terms derjenige des $A_{1}$ Terms, und gleichzeitig ist das Dehnungssignal aus dem ersten Reihenglied, der erwünschte Meßwert für den Spannungsintensitätsfaktor, annähernd maximal, so daß keine wesentlichen Aufösungseinbußen zu verzeichnen sind. Ergänzend sei vermerkt, daß das nächst höhere Reihenglied, der $B_{1}$-Term, Nullstellen lediglich bei ganzzahligen Vielfachen von $\pi$ (Extrema bei $(2 n+1) \pi / 2, n \in \mathbb{Z}$ ) hat, also eine weitere Genauigkeitsverbesserung über diesen Term ausgeschlossen ist.

Für die Applikation eines Dehnungsmeßstreifens unter den optimalen Winkeln $\alpha_{\mathrm{opt}}$ und $\varphi_{\text {opt }}$ verschwindet somit das zweite Reihenglied und das dritte Reihenglied verschwindet unter unwesentlichem Verlust an Signalhöhe ebenfalls. Die Auswertung eines Dehnungssignals pro Meßstelle liefert folglich eine dreiparametrige Lösung.

Bei der Festlegung der Applikationsdistanz zur Gewährleistung eines optimalen Meßabstandes müssen neben der Berücksichtigung der Störterme der Spannungsverteilung weitere Abwägungen angestellt werden. Diese resultieren teilweise aus simplen 


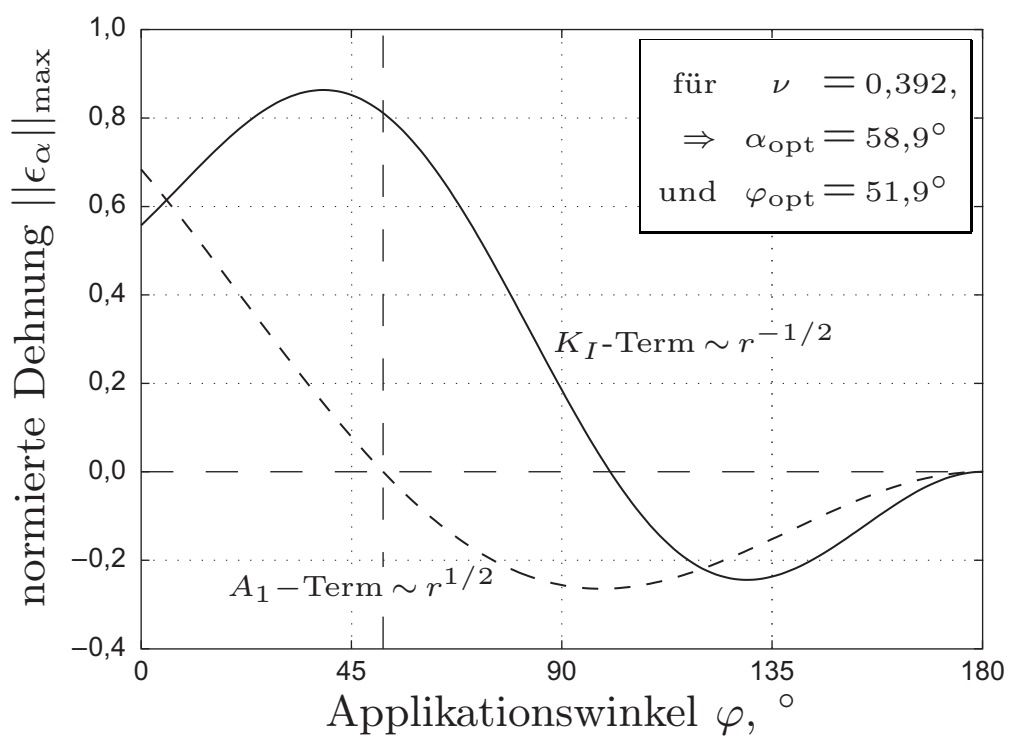

Abb. 22: Normierte Anteile der Reihenglieder des Dehnungsfeldes bei geneigter DMSApplikation im Bereich oberhalb des Ligaments bzw. des Risses (beispielhaft für das Modellmaterial Epoxidharz Araldit B).

geometrischen Beziehungen. Sowohl der Dehnungsmeßstreifen als auch die Rißspitze werden zur Vereinfachung als punktförmig angenommen. Wegen der tatsächlich graduell über die Fläche des Dehnungsmeßstreifens variierenden Dehnung (s. [136]) und der räumlichen Ausdehnung der Prozeßzone kann eine Korrektur der Dehnungsmeßstreifenposition (speziell für geringe Werte der Applikationsdistanz) erforderlich sein. Die Korrektur der Ausdehnung der Prozeßzone wird im allgemeinen über eine Anpassung der Rißspitzenposition vorgenommen: nach Irwin wird hierbei eine effektive Rißlänge $a_{\text {eff }}=a+r_{p}$ bestimmt, indem der Rißlänge einmal der Betrag des Prozeßzonenradius zugeschlagen wird. Allerdings liegen im vorliegenden Fall beide Korrekturen lediglich im Bereich von wenigen Zehntel Millimetern und darunter, sie sind somit zu gering um bei der praktischen Anwendung berücksichtigt zu werden. Dieses Ergebnis wird ebenfalls im Hinblick auf die Anforderungen des Kleinbereichsfließen, hier bezogen auf die Applikationsdistanz (die eine der relevanten Dimensionen gemäß Abschnitt 2.1.4 darstellt, vergl. auch Gl.10) verwendet.

Weiterhin ist der endliche Kerbradius von Bedeutung. Nach Creager und Paris [15] sind die Ergebnisse für das Spannungsfeld eines Risses auf schlanke Kerben übertragbar, wenn mit $\rho \ll r \ll a$ der Kerbradius $\rho$ klein ist im Verhältnis zum Meßabstand $r$ und dieser klein gegenüber der Rißlänge $a$ sowie allen anderen relevanten Dimensionen. Die mathematische Beschreibung der Kerbe erfolgt am besten durch Kegelschnitte (Kurven zweiter Ordnung), wie in Abb.23 am Beispiel der Ellipse dargestellt. Die Abbildung zeigt drei Risse, einen Linienriß mit scharfer Rißspitze (Abb.23a - zur Gerade entartete Ellipse) und zwei Kerben mit abgerundeter/abgestumpfter Spitze (Abbn. 23b - allgemeine elliptische Form und 23c - Kreis). Allgemein ist der Krüm- 


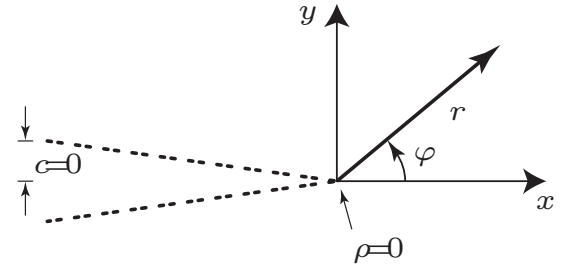

a) Scharfer Riß

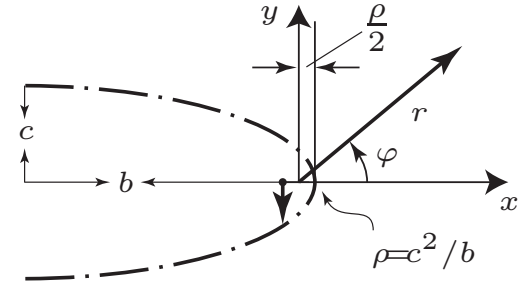

b) Elliptische Kerbe

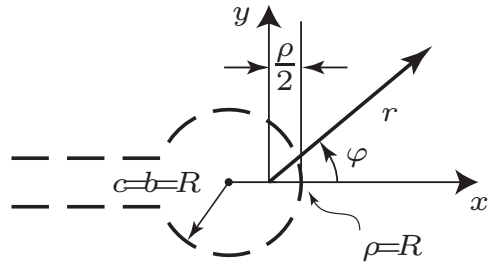

c) Kreisförmige Kerbe

Abb. 23: Ursprung des Spannungs-/Dehnungsfeldes (nach [24]).

mungsradius am Ligament/der Stelle stärkster Krümmung mit $\rho=c^{2} / b$ gleich dem zugehörigen Halbparameter. Für nicht entartete Ellipsen liegt dieser Wert zwischen $0<\rho<R$, dem Kreisradius. Zur Bestimmung des Spannungsfeldes von Kerben wird der Spannungsursprung als um einen halben Krümmungsradius in den Hohlraum der Kerbe hineinverlegt angenommen (s. Abb. 23). Das resultierende Spannungsfeld wird vollständig durch Spannungsintensitätsfaktoren charakterisiert und ist in Bezug auf den hydrostatischen Spannungszustand gleich dem eines scharfen Risses. Für große Abstände von der Rißspitze ist auch die Spannungsverteilung mit der des scharfen Risses identisch, Spannungsumlagerungen machen sich nur in der Nähe der Kerbspitze bemerkbar $(r / \rho<10,[139])$. Für die Applikation von Dehnungsmeßstreifen an Kerbspitzen wird im Rahmen der angestrebten Genauigkeit der um $\rho / 2$ korrigierte Spannungsursprung verwendet. Weitere geometrische Einflüsse, insbesondere solche, die durch die Ausdehnung der Prozeßzone (s. o.) hervorgerufen werden, werden nicht berücksichtigt [140].

Für Zug(Modus-I)-Beanspruchungen wird der Spannungsintensitätsfaktor somit auch bei endlichem Meßpunktabstand $r$ mit hoher Genauigkeit aus der mittels eines Dehnungsmeßstreifens gemessenen Dehnung $\epsilon_{\mathrm{DMS}}$ nach Dally und Sanford [136] bestimmt durch:

$$
K_{I}=\epsilon_{\mathrm{DMS}} E \sqrt{2 \pi r} \hat{f}(\nu, \alpha, \varphi)
$$

mit: $\quad \hat{f}(\nu, \varphi, \alpha)=\left[\frac{1-\nu}{1+\nu} \cos \frac{\varphi}{2}+\frac{1}{2} \sin \varphi\left(\cos \frac{3 \varphi}{2} \sin 2 \alpha-\sin \frac{3 \varphi}{2} \cos 2 \alpha\right)\right]^{-1}$,

wobei für statische Rißspitzen, wie hier beschrieben, die Funktion $\hat{f}(\varphi, \alpha, \nu)\left(\hat{f}=f^{-1}\right.$, vergl. Gl. 39) eine Konstante darstellt. Der Spannungsintensitätsfaktor $K_{I}$ und die gemessene Dehnung $\epsilon_{\mathrm{DMS}}$ sind folglich direkt zueinander proportional.

Für Scher(Modus-II)-Beanspruchungsbedingungen gelten die vorstehenden Grundsätze entsprechend, wiederum ausgehend von Gl. (36) ergibt sich das Rißspitzendeh- 
nungsfeld nach Bürgel [138] zu:

$$
\begin{aligned}
\epsilon_{x}^{I I}=\frac{1}{E}\left[\frac{K_{I I}}{\sqrt{2 \pi r}}\left(-\frac{2}{1+\nu} \sin \frac{\varphi}{2}-\frac{1}{2} \sin \varphi \cos \frac{3 \varphi}{2}\right)+\widetilde{B_{0}} r^{0}\right. \\
\left.+\widetilde{A_{1}} r^{\frac{1}{2}}\left(\frac{2}{1+\nu} \sin \varphi 2+\frac{1}{2} \sin \varphi \cos \frac{\varphi}{2}\right)+\cdots\right] \\
\epsilon_{y}^{I I}=\frac{1}{E}\left[\frac{K_{I I}}{\sqrt{2 \pi r}}\left(\frac{2 \nu}{1+\nu} \sin \frac{\varphi}{2}+\frac{1}{2} \sin \varphi \cos \frac{3 \varphi}{2}\right)-\nu \widetilde{B_{0}} r^{0}\right. \\
\left.\quad+\widetilde{A_{1}} r^{\frac{1}{2}}\left(-\frac{2}{1+\nu} \sin \varphi 2-\frac{1}{2} \sin \varphi \cos \frac{\varphi}{2}\right)+\cdots\right] \\
\gamma_{x y}^{I I}=\frac{2(1+\nu)}{E}\left[\frac{K_{I I}}{\sqrt{2 \pi r}}\left(\cos \frac{\varphi}{2}-\frac{1}{2} \sin \varphi \sin \frac{3 \varphi}{2}\right)\right. \\
\left.+\widetilde{A_{1}} r^{\frac{1}{2}}\left(\cos \frac{\varphi}{2}+\frac{1}{2} \sin \varphi \sin \frac{\varphi}{2}\right)+\cdots\right] .
\end{aligned}
$$

Wobei im allgemeinen die Koeffizienten $\left(\widetilde{A_{i}}, \widetilde{B_{i}} \neq A_{i}, B_{i}.\right)$ in Modus-I und Modus-II verschieden sind.

Des weiteren ist die Dehnung in Meßrichtung eines Dehnungsmeßstreifens nach Bürgel et al. [141] gegeben durch:

$$
\begin{aligned}
& \epsilon_{\alpha}^{I I}=\frac{1}{E}\left\{\frac { K _ { I I } } { \sqrt { 2 \pi r } } \left[-\frac{1-\nu}{1+\nu} \sin \frac{\varphi}{2}-\left(\sin \frac{\varphi}{2}+\frac{1}{2} \sin \varphi \cos \frac{3 \varphi}{2}\right) \cos 2 \alpha\right.\right. \\
& \left.+\left(\cos \frac{\varphi}{2}-\frac{1}{2} \sin \varphi \sin \frac{3 \varphi}{2}\right) \sin 2 \alpha\right] \\
& +\widetilde{B_{0}} r^{0}\left[\frac{1-\nu}{1+\nu}+\cos 2 \alpha\right] \\
& +\widetilde{A_{1}} r^{\frac{1}{2}}\left[\frac{1-\nu}{1+\nu} \sin \frac{\varphi}{2}+\left(\sin \frac{\varphi}{2}+\frac{1}{2} \sin \varphi \cos \frac{\varphi}{2}\right) \cos 2 \alpha\right. \\
& \left.+\left(\cos \frac{\varphi}{2}+\frac{1}{2} \sin \varphi \sin \frac{\varphi}{2}\right) \sin 2 \alpha\right] \\
& +\cdots\}
\end{aligned}
$$

Zur Steigerung der Genauigkeit der Messung wird unter Scher(Modus-II)-Bedingungen analog dem vorstehend geschilderten Verfahren vorgegangen. Hierbei ist nach Bürgel [141]:

$$
\alpha_{\mathrm{opt}}^{I I}=\alpha_{\mathrm{opt}}^{I},
$$


so daß wie unter Zug(Modus-I)-Bedingungen bei entsprechender Applikation der $\widetilde{B_{0}}$ Term eliminiert wird*. Jedoch ergeben sich für den zweiten Störterm, den $\widetilde{A_{1}}$-Term, für $\varphi\left(\alpha_{\mathrm{opt}}\right)$ in einem für Festkörper realistischen Poissonzahlbereich ausschließlich für die Rißufer Nullstellen, so daß hier im Rahmen der Optimierung lediglich die Minimierung dieses Terms erreicht wird. Der optimale Winkel $\varphi_{\text {opt }}^{I I}$ folgt aus der Differentiation der Spannungsentwicklung (für den entsprechenden optimalen Applikationswinkel, Gl. (44), nach der zweiten Stelle abgebrochenen): $\mathrm{d} \epsilon_{\alpha} / \mathrm{d} \varphi=0$ (die resultierenden optimalen Winkel liegen - wiederum für übliche Poissonzahlen - nur wenige Grad oberhalb des Ligaments, siehe Anhang C). Dennoch liefert nach Bürgel [141] die optimierte Methode auch für Scher(Modus-II)-Bedingungen mit:

$$
K_{I I}=\epsilon_{\mathrm{DMS}} E \sqrt{2 \pi r} \bar{f}(\nu, \alpha, \varphi)
$$

und:

$$
\begin{aligned}
\bar{f}(\nu, \varphi, \alpha)=\left[-\frac{1-\nu}{1+\nu} \sin \frac{\varphi}{2}\right. & -\left(\sin \frac{\varphi}{2}+\frac{1}{2} \sin \varphi \cos \frac{3 \varphi}{2}\right) \cos 2 \alpha \\
+ & \left.\left(\cos \frac{\varphi}{2}-\frac{1}{2} \sin \varphi \sin \frac{3 \varphi}{2}\right) \sin 2 \alpha\right]^{-1}
\end{aligned}
$$

sehr genaue, verbesserte Ergebnisse für den Spannungsintensitätsfaktor. Festzuhalten ist, daß in jedem Fall der erste Störterm, der $B_{0}$-Term ( $T$-Stress) nicht auftritt.

\subsubsection{Dehnungsmeßstreifen-Ketten-Verfahren bei laufenden Rissen}

Für Rißausbreitungsvorgänge, betrachtet in einem mit $\dot{x}=v_{c}$ (s. Abb. 24a) an der Rißspitze mitbewegten Koordinatensystem, ergeben sich für ortsfeste Dehnungsmeßstreifen Signale wie in Abb. 24b dargestellt: Bei der Annäherung an den Dehnungsmeßstreifen steigt das Signal an und fällt wieder ab, nachdem die Rißspitze den Dehnungsmeßstreifen passiert hat. Die Parameter $r$ und $\varphi$ sind hierbei nicht konstant, dadurch verschwindet bei Verwendung der optimalen Dehnungsmeßstreifenorientierung zwar der $B_{0}$-Term wie bei stationären Rissen (Beziehungen für $\alpha$ und $\alpha_{\text {opt }}$ gelten unverändert), die vollständige Elimination des $A_{1}$-Terms ist jedoch nicht möglich ( $\varphi$-Abhängigkeit, vergl. Gl. 39). Die Bestimmung von Spannungsintensitätsfaktoren/Rißlaufzähigkeiten aus solchen Dehnungsmeßstreifensignalen sowie die entsprechenden Genauigkeitsbetrachtungen für Rißlaufereignisse sind daher komplizierter als für den statischen Fall.

Das Meßsignal trägt beide gesuchte Informationen: die der Rißlaufgeschwindigkeit und die des Spannungsintensitätsfaktors bzw. des kritischen Wertes hiervon, der Rißlaufzähigkeit. Die Rißlaufgeschwindigkeit bestimmt die Signalweite und die Spannungsintensität/Rißlaufzähigkeit die Signalhöhe: Für jede Kombination von Rißlaufzähigkeit und Geschwindigkeit ergibt sich eine charakteristische Kurvenform, jedoch sind hier ebenfalls die Einflüsse der Störterme zu betrachten. Daraus ergeben sich für

\footnotetext{
*in Modus-II-Reihenentwicklung nach Williams nicht vorhanden [3].
} 


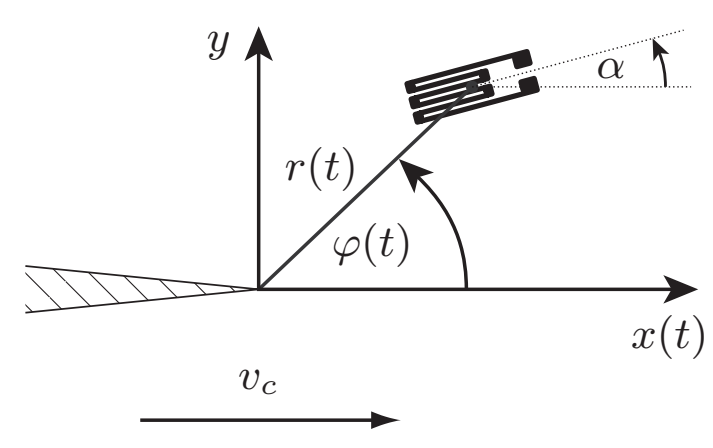

a)

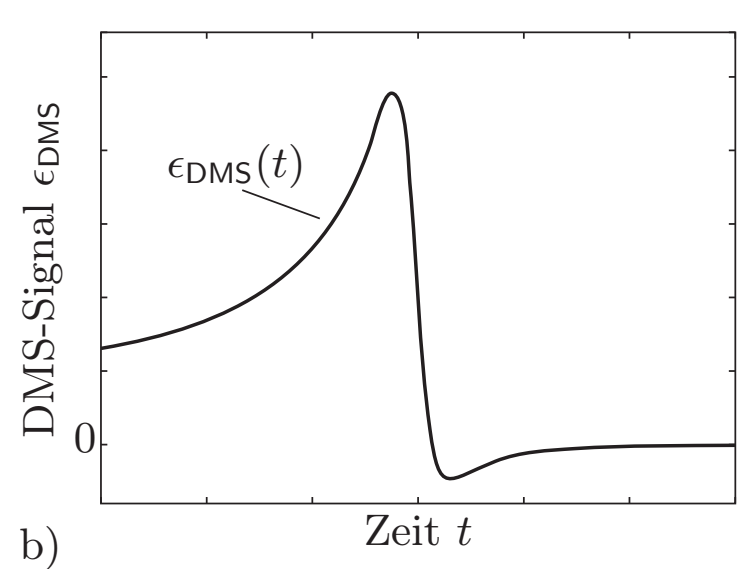

Abb. 24: Dehnungsbestimmung bei propagierender Rißspitze, schematisch: a) Applikationsparameter und b) Charakteristik des Meßsignals.

eine Auswertung der Experimente durch die Analyse der Signalform zwei Probleme. Zum einen sind die Geschwindigkeitsverläufe, die sich für die in den Experimenten erzeugten Rißlaufereignisse einstellen nicht gleichförmig ( $v_{c} \neq$ const.) - ein beschleunigter Rißausbreitungsprozeß ist vielmehr häufig sogar beabsichtigt - und die Signalform bildet in integraler Weise den gesamten Bereich der Rißlaufgeschwindigkeiten im Meßintervall ab. Da sich bei beschleunigenden Rissen, zum zweiten, selbst für geringe Einflüsse von $A_{1}$ signifikante Änderungen in Kurvenform und Amplitude ergeben und somit eine Mehrdeutigkeit auftritt, wie in Anhang B gezeigt, eignet sich eine adaptive Kurvenanpassung einzelner Meßsignale oder die Verwendung von Modellkurvenatlanten nicht zur Auswertung der Experimente [137]. Stattdessen wurde eine Technik entwickelt, die auf jeweils zwei Dehnungsmeßstreifensignalen basiert, wie im folgenden erläutert.

Es werden Dehnungsmeßstreifen-Ketten verwendet, die in der im vorangegangenen Abschnitt geschilderten Art und Weise längs des prospektiven Rißpfades appliziert werden. Für zwei Dehnungsmeßstreifen einer solchen Kette, an denen die Rißspitze nacheinander vorbeiläuft ergibt sich, wie in Abb. 25 gezeigt, ein zeitlicher Abstand $\Delta t$ der beiden Signale. Nach der Beziehung (nach Dally und Sanford, siehe [136]):

$$
K_{I}=\epsilon_{\mathrm{DMS}} E \sqrt{2 \pi r}[f(\varphi, \nu)]^{-1}
$$

unter Berücksichtigung der material- und geometrieabhängigen Funktion $f$ wird die Rißlaufzähigkeit $K_{I D}$ als kritischer Wert des Spannungsintensitätsfaktors bei der maximalen vom Dehnungsmeßstreifen gemessenen Dehnung bestimmt. Der Abstand zweier charakteristischer Punkte der Meßkurven zusammen mit dem Abstand der Dehnungsmeßstreifen liefert die Rißgeschwindigkeit. Zusätzlich wird an der Starterkerbe der Initiierungswert der Bruchzähigkeit $\left(v_{c}=0\right)$ bestimmt. Unter Einsatz einer Vielzahl von Dehnungsmeßstreifen werden der gesamte Geschwindigkeitsverlauf, von der Initiierung bis in den Bereich möglicherweise sehr hoher Rißlaufgeschwindigkeiten und gleichzeitig die zugeordneten Rißlaufzähigkeiten während eines Rißlaufer- 


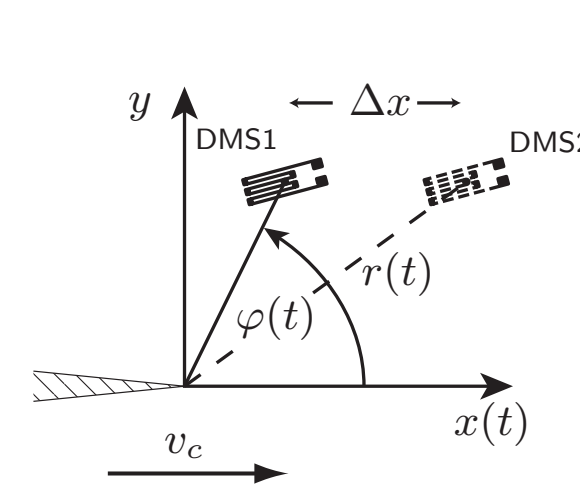

a)

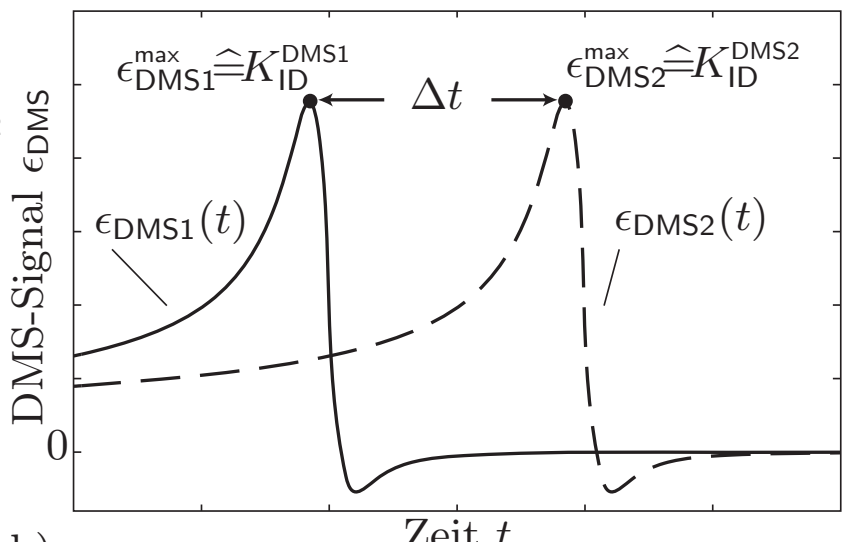

b)

Abb. 25: Prinzip der Bestimmung von Rißlaufzähigkeit $\left(K_{I D}\right)$ und Rißlaufgeschwindigkeit $\left(v_{c}\right)$ mit dem Dehnungsmeßstreifen-Ketten-Verfahren.

eignisses dokumentiert. Mit den eingesetzten Kurzzeit-Datenerfassungsgeräten sind Abtastraten im ns-Sekunden-Bereich möglich. Interpolation wird verwendet, wo dies notwendig/möglich erscheint. Die so mit Dehnungsmeßstreifen-Ketten gemessenen Rißgeschwindigkeiten werden durch einen optischen Vergleich mittels Hochgeschwindigkeitsfotographie bestätigt, der in einigen Fällen gleichzeitig an derselben Probe durchgeführt wird. Dazu wird eine Cranz-Schardin-Hochgeschwindigkeitskamera (ausführlich beschrieben u. a. in [138]) eingesetzt. Mit dieser Kamera wird eine Folge von Einzelbildern mithilfe extrem schnell zündender und abklingender Blitzlichter aufgenommen, wobei während des Experimentes das Labor verdunkelt wird. Aufgrund der Opazität des Materials wird die Probenoberfläche in einem speziellen Verfahren verspiegelt und die Cranz-Schardin-Kamera in einer Reflexionsanordnung betrieben. Die als Spiegel auf der Probenoberfläche aufgebrachte Schicht ist von so geringer Dicke, daß eine Beeinträchtigung des Rißausbreitungsprozesses ausgeschlossen ist (siehe Anhang D).

Generelle Aussagen zur Genauigkeit der Methode in der Anwendung bei Rißlaufereignissen zu treffen ist aufgrund vielfacher Einflüsse kompliziert. Die Genauigkeit hängt durch die stärkeren Störtermeinflüsse in gesteigertem Maße von den Eigenschaften des Materials oder der Probengeometrie ab und ist insgesamt als geringfügig schlechter einzustufen als im stationären Fall. Es liegt eine Mehrdeutigkeit der Ergebnisse vor (s. Anhang B). Der Einfluß der $\varphi$-Variation auf das Meßergebnis läßt sich anhand von Modellexperimenten mit konstanter Rißgeschwindigkeit und konstanter Rißlaufzähigkeit an dem Epoxidharz Araldit-B über Koeffizientenvergleich mit einer analytischen Lösung mit weniger als 10 Prozent beziffern.

Als optimale Distanz $r$ des Dehnungsmeßstreifens zur Rißspitze wurde unter Berücksichtigung der o. a. Gesichtspunkte ein Abstand von $5 \mathrm{~mm}$ bestimmt. Bei der Auswahl der Dehnungsmeßstreifen wurde Faktoren der Meßtechnik, wie Signalstärke oder Applizierbarkeit und materialseitigen, wie etwa der Überdeckung eines repräsentativen, homogenen Flächenelements oder der Kalorimetrie Rechnung getragen. 
Durch die Eliminierung bzw. Minimierung der Störtermeinflüsse (Wegfall des $B_{0^{-}}$ Terms/T-Stresses) werden auch bei dieser Methode Spannungsintensitätsfaktoren mit hoher Genauigkeit bestimmt.

\subsection{Methoden zur Erzeugung von Rißlaufereignissen unter Laborbedingungen von der Initiierung bis in den Bereich der maximalen Geschwindigkeit}

Die Ausbreitungsgeschwindigkeit eines Rißlaufereignisses wird, wie in Abschnitt 2.3.4 beschrieben, durch das jeweils vorliegende Energieangebot bzw. die jeweils vorliegende Energieanlieferungsrate gesteuert. Mittels des Einsatzes geeigneter Belastungstechniken wiederum wird in den Laborexperimenten das für den jeweils angestrebten Geschwindigkeitsbereich notwendige Energieniveau generiert, um den Rißausbreitungsprozeß von der Initiierung bis hin zu sehr hohen Geschwindigkeiten zu untersuchen. Da im Gegensatz zu langsamer Rißausbreitung gerade die Erzeugung schneller Rißausbreitung eine Herausforderung darstellt, wird vor allem auf das hierfür benötigte Energieüberangebot eingegangen, durch das stark steigende Spannungsintensitätsfaktoren oder Energiefreisetzungsraten zur Verfügung gestellt und letztlich maximale Rißausbreitungsgeschwindigkeiten erreicht werden. Zu Beginn des Abschnitts wird im folgenden jedoch beschrieben, wie - auch für den Bereich der langsamen, unter quasistatischen Beanspruchungsbedingungen erzeugten Rißausbreitung - geeignete Techniken eingesetzt werden, mit denen das Energieangebot und somit die Ausbreitungsgeschwindigkeiten auch auf diesem Geschwindigkeitsniveau variiert wird.

\subsubsection{Einflüsse der Probengeometrie und der Belastungsart auf die Erzeugung von Rißlaufereignissen bei quasistatischer Bean- spruchung}

Für bruchmechanische Laborproben hängen die aus elastischer Dehnungsenergie resultierenden Rißspitzen-Beanspruchungsfelder wesentlich von den im Experiment eingestellten Randbedingungen ab. Einer der maßgeblichen Faktoren ist hierbei die Art der Beanspruchungseinleitung, die bei quasistatischen Experimenten unterschieden wird in Bedingungen konstanter Last bzw. konstanter Verschiebung (siehe Abschnitt 2.1.5). Im Experiment wird dies durch die Vorgabe einer konstanten Last(,soft machine") bzw. einer konstanten Verformungsrate (,stiff machine") zumeist unter Verwendung servo-hydraulisch geregelter Belastungssysteme realisiert. Des weiteren sind die Randbedingungen, die aus der jeweiligen Probengeometrie herrühren, von Bedeutung. Für unterschiedliche Probengeometrien sind die Beanspruchungscharakteristiken unter weggeregelter Beanspruchung (Bedingungen konstanter Verschiebung) in Abb. 26a bis Abb. 26d und unter lastgeregelter Beanspruchung (Bedingun- 
gen konstanter Last) in Abb. 26e und Abb. 26f für eine Auswahl häufig verwendeter Grundformen (eine ausführliche Beschreibung unterschiedlicher Probentypen findet sich z. B. in [1]) als Funktion der Rißlänge skizziert (siehe auch [45]). Die Gegenüberstellung zeigt, dass sich die Rißausbreitung von einem Startriß der Länge $a_{0}$ für die in Abb. 26a angegebene Probenformen in einem Feld steigender Beanspruchungsgrößen vollzieht. Dies gilt ebenfalls, wenn auch in geringerem Maße und nur für eine begrenzten Teil des Rißlaufweges, für die in Abb. 26b dargestellten Konfigurationen. Wohingegen für die restlichen angegebenen Fälle konstanter Verschiebung
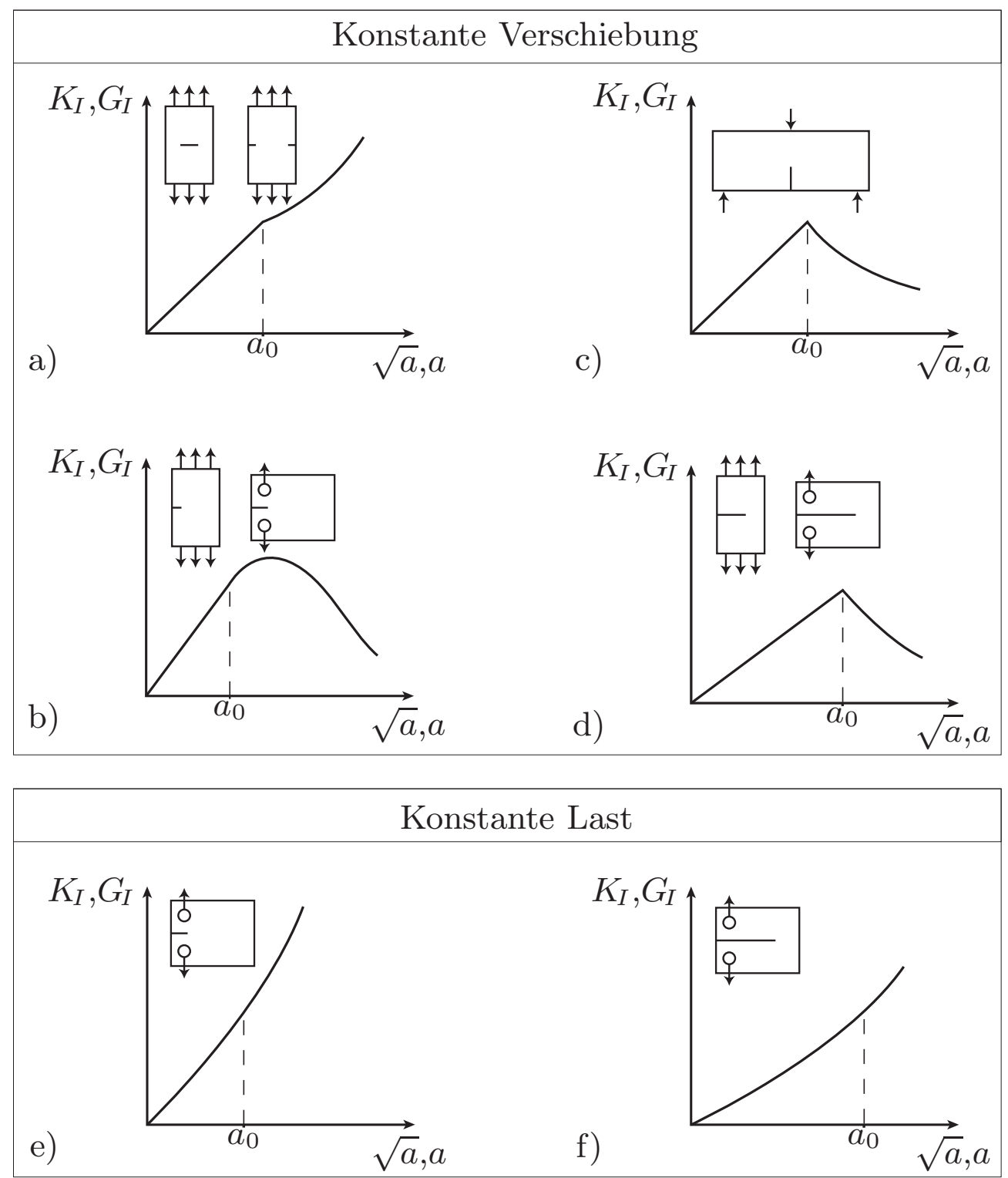

Abb. 26: Unterschiede der Beanspruchungs-Rißlängen-Charakteristik $\left(K_{I}(\sqrt{a})\right.$ und $\left.G_{I}(a)\right)$ für verschiedene, grundlegende Probengeometrien unter Beanspruchungsbedingungen konstanter Verschiebung (a-d) und konstanter Last $(\mathrm{e}, \mathrm{f})$, schematisch nach [142]. 
(Abb. 26c,d) der Rißfortschritt nach dem Beginn der Rißausbreitung unter der Bedingung abfallender Beanspruchungen erfolgt. Die letztgenannten Probentypen sind folglich für die Untersuchung langsamer, subkritischer Rißausbreitung geeignet. Zur Durchführung solcher Experimente werden im Rahmen dieser Arbeit SENB-Proben (single edge notch bend) in Dreipunktbiegeanordnung eingesetzt (Abb. 26c). Diese Festlegung erfolgte $u$. a. deshalb, weil der - durch das äußerst spröde Materialverhalten von Gestein stets nicht unerhebliche - Aufwand bei der Probenfertigung für diesen Probentyp vergleichsweise gering ist, da beispielsweise das Einbringen von Bohrlöchern zur Befestigung der Probenhalterung entfällt.

Für Experimente zur Untersuchung schneller Rißausbreitung ist prinzipiell die Verwendung konstanter Lastraten vorteilhaft, da sich hierbei gegenüber der Methode konstanter Verschiebung grundsätzlich höhere Beanspruchungsraten einstellen. Die Beanspruchungs-Rißlängen-Charakteristik bei konstanter Last gleicht für eine Vielzahl der gängigen Probengeometrien (und für sämtliche der in Abb. 26 angegebenen Proben) in ihrer Gestalt derjenigen der in Abb. 26e,f stellvertretend dargestellten Kurven (siehe [142]). Diese Methode wird im Rahmen der vorliegenden Arbeit im Zusammenhang mit quasistatischer Lasteinleitung nicht eingesetzt. Um eine Änderung bzw. eine Steigerung der Bruchausbreitungsgeschwindigkeit zu erreichen, werden stattdessen alternative Möglichkeiten aus den Einflüssen von Probenform und Beanspruchung genutzt. Hierzu zählt die Variation der Rißlänge. Bei geringerer Rißlänge ergibt sich ein höheres Anfangsenergieniveau, wie der Vergleich der Kurven aus Abb. 26b und Abb. 26d bzw. Abb. 26e und Abb. 26f zeigt.

Die aufgeführten Prinzipien sind grundsätzlich auf Materialien übertragbar, die, wie Gestein, eine steigende $R$-Kurve (s. Abs. 2.2) aufweisen. Um in solchen Materialien hohe Geschwindigkeiten bei der Rißausbreitung bereits nach kurzen Rißlaufwegen zu erreichen, ist - über die vorstehend beschriebenen Methoden hinaus - eine weitere Steigerung des Energieniveaus bei der Initiierung nötig.

Eine weitere Möglichkeit zur Rißgeschwindigkeitserhöhung bietet die Verwendung stumpfer Starterkerben, für die im Vergleich mit scharfen Anrissen größere Beträge von Dehnungsenergie in der Probe gespeichert werden bevor der Rißlaufprozeß initiiert. Den gegenteiligen Effekt hätte der Einsatz sogenannter Chevron-notchStarterkerben, bei denen die Rißfront nach der Initiierung von einer schlanken Rißspitze an Breite zunimmt, wodurch der bei sehr geringen Spannungsintensitäten initiierende Riß bei der Ausbreitung einen ansteigenden Bruchwiderstand überwinden muß. Solche Starterkerben werden, wie bei der Standardmethode zur Bestimmung von Bruchzähigkeiten in Gestein, gezielt eingesetzt, um langsame, subkritische Rißlaufereignisse zu erzeugen [109, 143,144]. An dieser Stelle sei nochmals darauf hingewiesen, daß gültige Bruchzähigkeiten nur für scharfe Rißspitzen vorliegen. In diesem Sinne sind bei Rißlaufereignissen, die von stumpfen Kerben initiiert wurden, zwar die Rißlaufzähigkeiten gültig, Initiierungszähigkeiten sind jedoch nur relativ vergleichbar und müssen dementsprechend gekennzeichnet werden. 


\subsubsection{Erzeugung schneller Rißausbreitung durch dynamische Be- anspruchung}

Zur schnellen Rißausbreitung ist ein Energieniveau erforderlich, das gemäß den Bedingung aus den Gln. (16) und (17) ein Energieüberangebot bereitstellt, für das die Bruchausbreitung nicht nur fortgesetzt wird, sondern zusätzlich beschleunigt verläuft (vergl. auch Gl. 23). Die hierfür benötigten Felder stark steigender Beanspruchungsgrößen $K$ oder $G$ entstehen bei Schlag-/Stoßbelastungsbedingungen. Bei einer solchen dynamischen Belastung bewirken die sich im Probenkörper ausbreitenden elastischen Wellen einen raschen Anstieg der Feldgrößen. Zudem ergibt diese spezielle Belastungsart eine kontinuierliche Energieanlieferung, aus der für jegliche Probenform ansteigende Beanspruchungsfelder $\mathrm{d} K / \mathrm{d} t$ bzw. $\mathrm{d} G / \mathrm{d} t$ resultieren, da hier $W^{a} \neq 0$ sowie $\mathrm{d} W^{a} / \mathrm{d} t \gg 0$ (vergl. Abs. 2.1.5) und somit insgesamt eine sehr hohe Beanspruchungsrate resultiert.

Im allgemeinen vollzieht das Testsystem, bestehend aus dem Stoßkörper und der zu untersuchenden Probe (s. Abb. 27a), angeregt durch den Stoßprozeß charakteristische Schwingungen (vergl. Abschnitt 2.3.3), deren Schwingungsdauer $\tau$ (s. Abb. 27b) im wesentlichen durch die Eigenfrequenz der Laborprobe bestimmt werden [84]. Dies sollte generell bei der Planung und Auswertung von Stoßexperimenten bedacht werden. Für die in dieser Arbeit im dynamischen Dreipunktbiegeversuch unter Stoßbelastung durchgeführten Hauptexperimente werden die Spannungsintensitäten (bzw. durch die Bestimmung der Maxima der Spannungsintensitäten die Bruchzähigkeiten) aus den lokalen Beanspruchungsfeldern an der Rißspitze bestimmt und nicht gemäß dem ASTM-Normprüfverfahren aus den äußeren Beanspruchungsparametern an der Lasteinleitungsstelle oder durch die Rißöffnung. Zu diesem Zweck werden Dehnungsmeßstreifen direkt entlang des prospektiven Rißpfades appliziert, d. h. in diesem Fall entlang des Ligaments. Beiträge aus verfälschenden Einflüssen abseits des Rißlaufweges werden so wesentlich reduziert $[77,80]$. Des weiteren weisen die untersuchten Proben aufgrund konzeptioneller Überlegungen (s. Abs. 4.1) ein großes Verhältnis

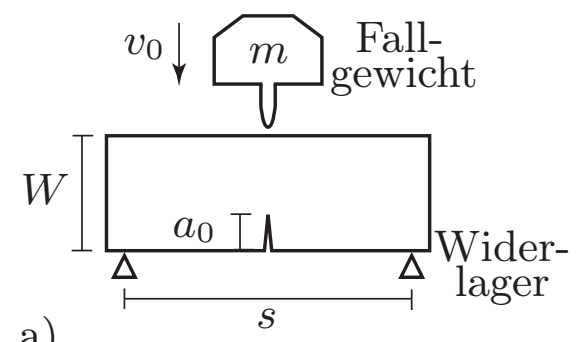

a)

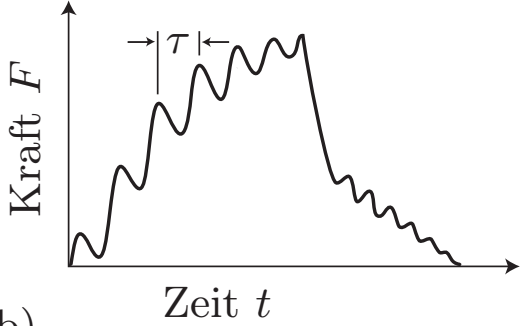

b)

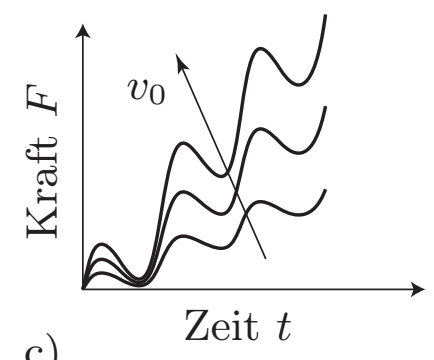

c)

Abb. 27: Prinzip des Schlagbiegeversuchs: a) Skizze der Fallgewichtsapparatur, b) Ausbildung von Schwingungen der Schwingungsdauer $\tau$ im Kraftsignal $F(t)$, schematisch und c) Steigerung der Beanspruchungsrate $F(t)$ bei steigender Stoßgeschwindigkeit $v_{0}$, gezeigt für den Anfangsbereich der Beanspruchung (vereinfacht nach [84]). 
von Probenhöhe $W$ (Abb.27a) zu Probenbreite bzw. zum Auflagerabstand $s$ auf. Daher sind diese Laborproben sehr steif und die resultierenden Schwingungsamplituden grundsätzlich gering. Zudem erfolgt die Rißinitiierung in Gestein aufgrund des spröden Materialversagens zu einem sehr frühen Zeitpunkt in der Beanspruchungsphase [145]. Die Schwingungsphänomene sind aufgrund ihres geringen Einflusses daher nicht zu berücksichtigen. Solche Schwingungsphänomene werden im Abschnitt $4.7 \mathrm{im}$ Zusammenhang mit einem Globalenergie-Verfahren diskutiert. Dieses Verfahren basiert auf einem standardisierten Prüfverfahren und unterliegt prinzipiell im Hinblick auf die Schwingungsproblematik denselben Gesetzmäßigkeiten, die sich bei den dort vorliegenden Probengeometrien und Testbedingungen im Gegensatz zu den hier zu Grunde liegenden stärker abbilden. Die für diese Initiierungsmethode resultierende Beanspruchung setzt sich aus inneren Anteilen durch Wellenausbreitung in der Probe und aus äußeren Anteilen durch Lasteinleitung und Widerlager zusammen (weitere Betrachtungen zur experimentellen Realisierung dynamischer Rißinitiierung finden sich in [84]). Das Energieniveau, das für den Rißausbreitungsprozeß zur Verfügung steht, wird durch die Fallhöhe bzw. durch die Auftreffgeschwindigkeit $v_{0}$ gesteuert (Abb. 27c).

\subsubsection{Einsatz von Scher(Modus-II)-Beanspruchungsbedingungen zur Erzeugung von Rißlaufereignissen mit hoher Bruchgeschwin- digkeit}

Eine Steigerung des Energieniveaus ergibt sich zudem durch Verwendung von ModusII-Initiierung. Für die an der Rißspitze gespeicherte elastische Energie $U_{p}$ (siehe [49]; vergl. auch Abschnitt 2.1.5) unter einer gemischten Beanspruchung aus Modus-I- und Modus-II-Anteilen ( $M M I$ bzw. $M M I I)$ gilt:

$$
\frac{U_{p, M M}}{U_{P M I}}=\left(K_{M M I}^{2}+\frac{2 \kappa+3}{2 \kappa-1} K_{M M I I}^{2}\right) \frac{1}{K_{P M I}^{2}},
$$

wobei $\kappa=(3-\nu) /(1+\nu)$ und $\kappa=3-4 \nu$ für ebenen Spannungszustand bzw. ebenen Dehnungszustand (vergl. Kapitel 2, S. 5). Zur besseren Vergleichbarkeit wurde eine Normierung auf reine Zug(Modus-I)-Beanspruchungsbedingungen ( $P M I: K_{M M I}=$ $\left.K_{P M I} K_{M M I I}=0\right)$ vorgenommen. Äquivalente Beanspruchungsbedingungen: $K_{P M I}=$ $K_{P M I I}=\sqrt{K_{M M I}^{2}+K_{M M I I}^{2}}$ werden vorausgesetzt. Wie in Abb. 28 dargestellt, übersteigt die Rißspitzendehnungsenergie unter reinen Scher(Modus-II)-Beanspruchungsbedingungen diejenige unter reinen Zug(Modus-I)-Beanspruchungsbedingungen mit äquivalentem Beanspruchungsbetrag um mehr als das doppelte. Dieses Ergebnis wurde durch Podleschny [146] verifiziert: Unter Anwendung spezieller Maßnahmen zur Steuerung des Initiierungsniveaus wurden experimentell äquivalente Bedingungen für den kritischen Beanspruchungszustand $\left(K_{I}^{i n i t}=K_{I I}^{i n i t}\right)$ erzeugt. Für unter Scher(Modus-II)-Bedingungen initiierte $\mathrm{Zug}$ (Modus-I)-Risse wurden entsprechend um den Faktor zwei höhere Ausbreitungsgeschwindigkeiten gegenüber Zug(ModusI)-Initiierung bestimmt. Dadurch bedingt ist auch die Ausdehnung der Prozeßzone 


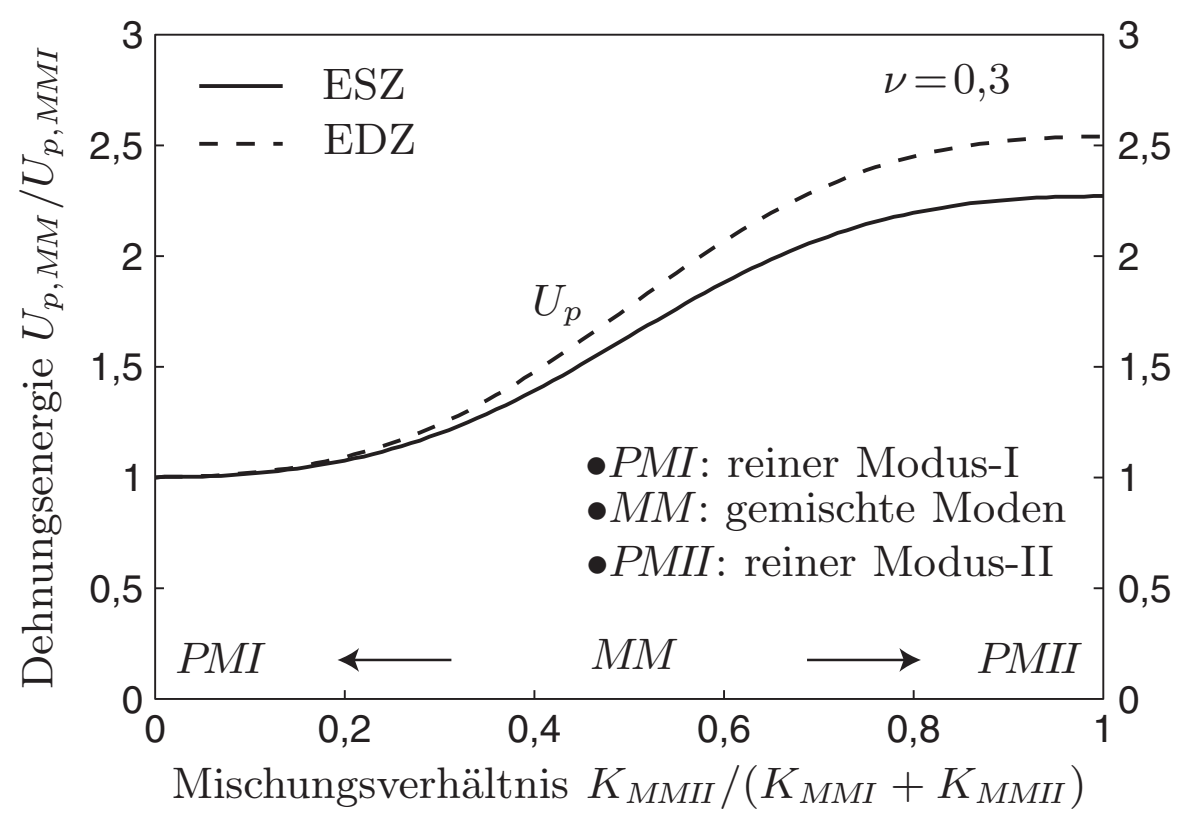

Abb. 28: Rißspitzendehnungsenergie $U_{p}$ als Funktion des Moden-Mischungsverhältnisses: Reine Beanspruchungsmoden liegen für Zug am linken Rand $\left(K_{M M I I} /\left(K_{M M I}+K_{M M I I}\right)=0\right)$ bzw. für Schub am rechten Rand $\left(K_{M M I I} /\right.$ $\left.\left(K_{M M I}+K_{M M I I}\right)=1\right)$ der Abszisse vor (nach [49]).

unter Modus-II-Beanspruchungsbedingungen größer als für Modus-I (vergl. Abb. 5). Ein sehr ähnliches Verhältnis ergibt sich für stumpfe Kerben [49].

\subsubsection{Einsatz dynamischer Scher(Modus-II)-Beanspruchungsbedin- gungen mit der LECEI-Technik zur Erzeugung von Rißlauf- ereignissen mit maximaler Bruchgeschwindigkeit}

Noch höhere Energieniveaus bzw. Beanspruchungsraten bei der Rißinitiierung werden erreicht, wenn Stoß- mit Scherbeanspruchungsbedingungen kombiniert werden. Unter dynamischen Scher(Modus-II)-Beanspruchungsbedingungen gelten die in Abschnitt 2.3 beschriebenen Beziehungen entsprechend.

Um dynamische Scher(Modus-II)-Beanspruchungsbedingungen experimentell zu realisieren, wird die LECEI-Technik (loading of edge çracks by edge inpact) eingesetzt, von Kalthoff eingeführt [147]. Bei dieser Methode wird der Probenkörper, wie in Abb. 29 skizziert, asymmetrisch mit einem Druckspannungspuls beaufschlagt, der durch ein mit einer Geschwindigkeit $v_{0}$ auf die Probe auftreffendes Projektil generiert wird. Die in dieser Arbeit verwendeten quadratischen Probenscheiben sind in halber Probenhöhe mit einer Starterkerbe versehen, die bis zur Mitte der Probe reicht. Die Probe wird so vor dem Beschleunigungsrohr positioniert, daß das gasdruckgetriebene Projektil oberhalb der Kerbe auftrifft. Infolge des Aufschlages des Projektils breitet sich oberhalb der Kerbe eine Druckwelle in der Probe aus, der un- 


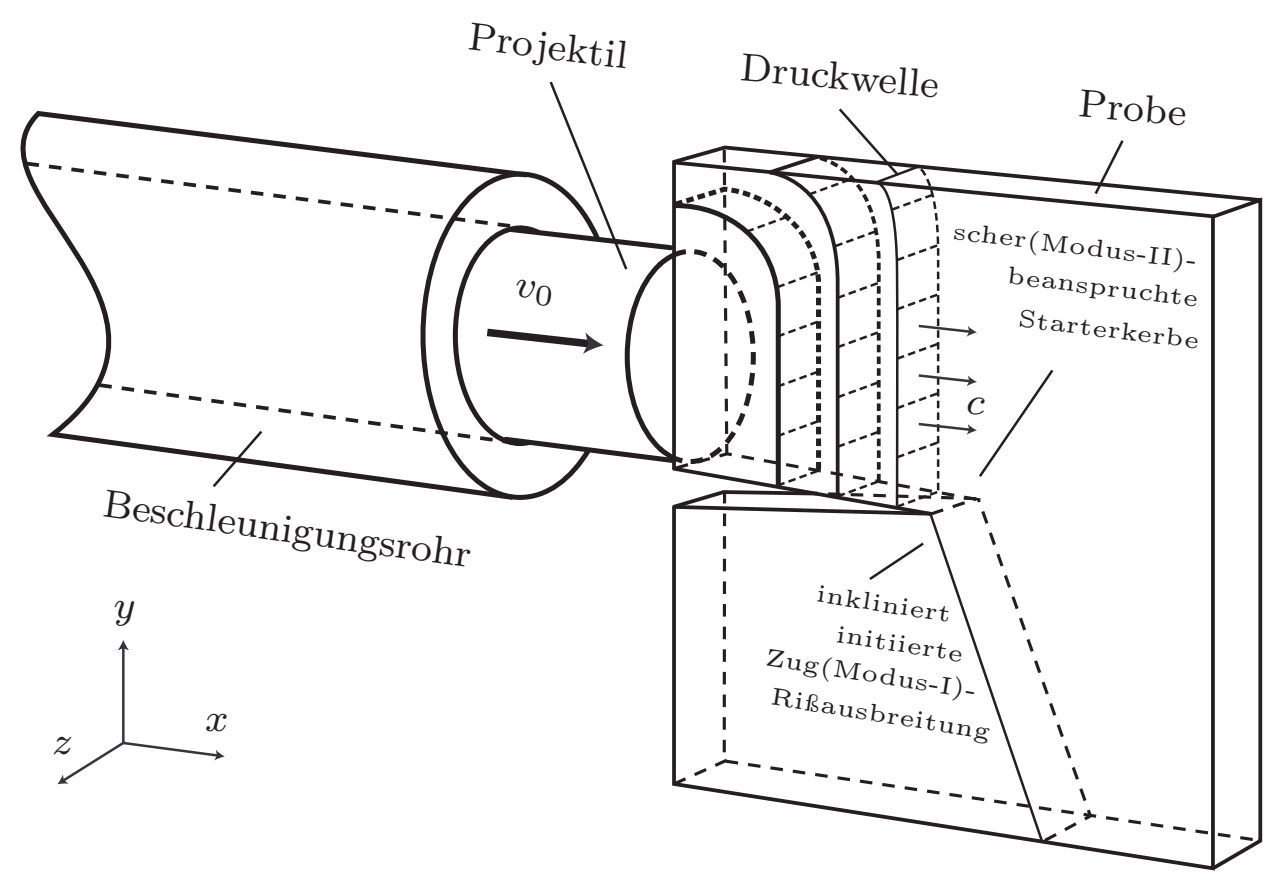

Abb. 29: Prinzipskizze des LECEI-Experimentes.

tere Teil der Probe bleibt zunächst spannungsfrei. Nach dem Eintreffen der Druckoder Schockwelle an der Kerbspitze erzeugt diese dort ein Spannungsintensitätsfeld, das im wesentlichen eine Charakteristik mit positivem Schermodus aufweist. Die Spannungsintensität wächst infolge der großen Druckgradienten in der Schockwelle sehr schnell an. Bei Erreichen der kritischen Spannungsintensität $K_{I I d}$ initiiert von der scherbeanspruchten Kerbspitze ein Zug(Modus-I)-Riß, der eine Inklination zum Ligament aufweist (s. Abschnitt 2.2.2, Seite 19). Der Verlauf des Experimentes ist hochdynamisch, d. h. der zeitliche Ablauf vom Projektilaufschlag bis zur Rißausbreitung erfolgt so schnell, daß auf eine Fixierung der Probe verzichtet werden kann. Um ideale Initiierungsbedingungen zu gewährleisten, wird die Probe auf einem justierbaren Probentisch exakt planparallel zur Stirnfläche des Projektils ausgerichtet. Nach Beendigung der Hauptzugrißimplementierung von der Starterkerbspitze kommt es zu vielfachen sekundären Rißereignissen, die meist von den Probenrändern ausgehen. Diese werden einerseits dadurch verursacht, daß bei der Reflexion der primären Druckwelle an den freien Rändern der Probe ein Phasensprung auftritt und die Probe unter den dadurch auftretenden Zugspannungen versagt. Andererseits kommt es nach der Reflektion an verschiedenen Rändern allgemein zu Überlagerungsphänomenen, die wiederum Rißereignisse initiieren können.

Das Ausbilden der Rißspitzenfelder ist anschaulich im Experiment selbst zu erfassen, wie in Abb. 30 mittels Kaustikenbildern gezeigt. Die Kaustik besteht aus einem Schattenfleck, der Kaustikenfläche, und einer lichtstarken Grenzkurve (zur schattenoptischen Kaustikenmethode in der experimentellen Spannungsanalyse siehe [87, 149]). 

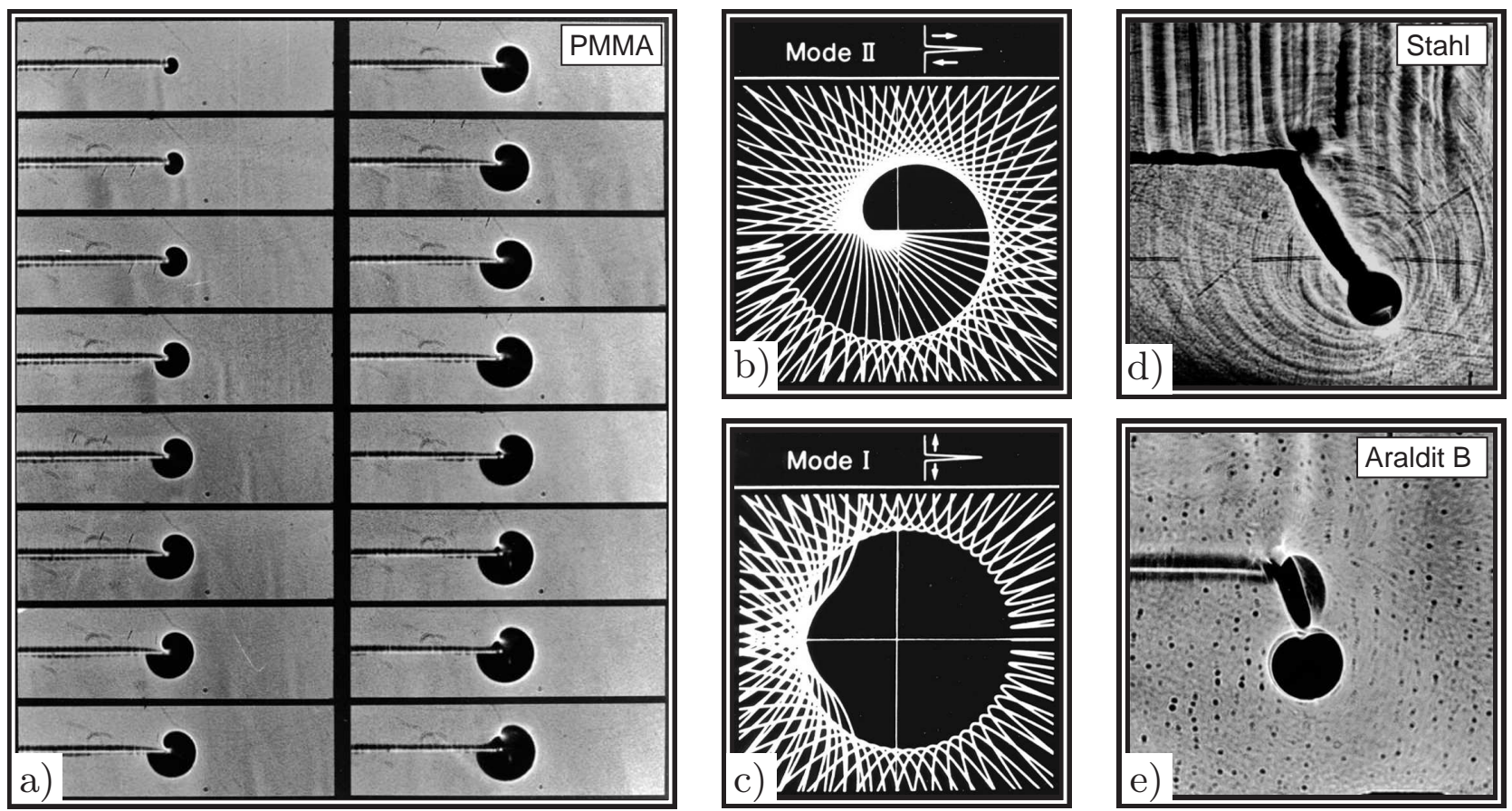

Abb. 30: Entwicklung der Beanspruchungsmoden im Verlauf des LECEIExperimentes, beobachtet mit der schattenoptischen Kaustikenmethode (zusammengestellt aus [148]).

Form und Größe der Kaustik werden durch die Beanspruchungsbedingungen an der Rißspitze bestimmt. Eine Serie von Kaustikenbildern, die während der Beanspruchungsphase im LECEI-Experiment mit einer Hochgeschwindigkeitskamera aufgenommen wurden (Probenmaterial: Polymethylmetacrylat, PMMA), ist in Abb. 30a angegeben. Der Vergleich mit der idealen Form einer Scher(Modus-II)-Kaustik des Lichtintesitätsbildes (Abb. 30b) zeigt, daß nach einer anfänglichen Aufbauphase eine weitgehend reine Scher(Modus-II)-Beanspruchung vorherrscht (in den letzten drei Bildern in Abb.30a ist aufgrund der einsetzenden Rißinitiierung oberhalb der Spitze des Starterrisses eine Veränderung an der Kaustik zu erkennen).

Die Kaustik eines von der Rißspitze initiierten Risses weist die gleiche Form wie das zur Referenz in Abb. 30c angegebene Zug(Modus-I)-Lichtintensitätsbild auf. Als Beispiel sind in den Abbn. 30d,e Hochgeschwindigkeitsphotos von Kaustiken an der Spitze von Zug(Modus-I)-Rissen für zwei unterschiedliche Materialien dargestellt (für Stahl und das Epoxidharz Araldit B). In den Bildern bildet sich zudem die Wellenausbreitung ab, besonders deutlich in Abb.30d zu erkennen. Einschränkend sei erwähnt, daß ein Teil der Initiierungsenergie (25\%) wegen der Richtungsänderung des Risses nicht für den Ausbreitungsprozeß zur Verfügung steht [146].

Angepaßt auf den speziellen experimentellen Rahmen der Arbeit, wurden zur Beurteilung der Initiierungsbedingungen Finite-Elemente-Modellberechnungen durchgeführt. Abbildung 31 zeigt das Spannungsfeld (von-Mises-Spannungen) für ein drei- 


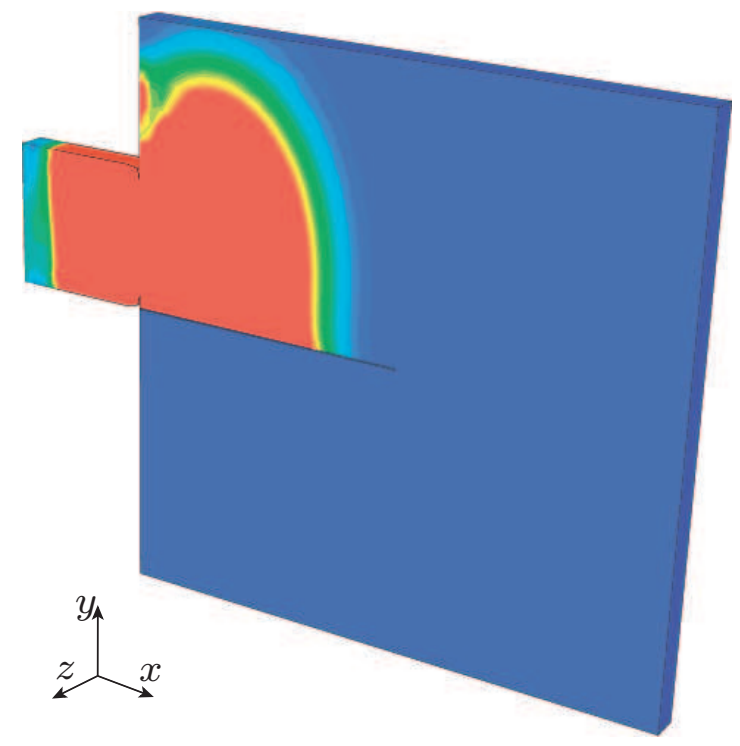

a)

Abb. 31: Finite-Elemente-Modell des LECEI-Experimentes: a) Ausbreitung der Druckwelle und b) Aufbau der Rißspitzenbeanspruchung.

dimensionales Modell mit einer abgerundeten Starterkerbe zu zwei verschiedenen Zeitpunkten. In Abb.31a ist die Druckwelle zu erkennen, die durch das Aufschlagen des Projektils in der Probe generiert wird. Das Rißspitzenspannungsfeld, das sich im weiteren Verlauf des Experimentes ausbildet, ist in Abb.31b dargestellt. Die Form dieses Feldes ist bezüglich der Amplitudenverteilung stark asymmetrisch (vergl. Abb.5). Eine bruchmechanische Auswertung (siehe Anhang E - dort wird auch das Modell vorgestellt) zeigt, daß diese Asymmetrie durch die endliche Ausbreitungsgeschwindigkeit der Druckwelle hervorgerufen wird. Dieser Effekt stellt, gerade bei Untersuchungen der Zug(Modus-I)-Rißausbreitung, keine wesentliche Beeinträchtigung dar. Auf den Betrag des Bruchwinkels ergibt sich allerdings ein Einfluß. Mit dem dynamisch Finite-Elemente-Modell wurden für den Zeitpunkt, zu dem die Spannungsintensitätsfaktoren kritische Werte annehmen, geringen Anteile einer Modus-I-Beanspruchung bestimmt. Bei den vorliegenden Materialparametern ist der Bruchwinkel daher um bis $\mathrm{zu} 5^{\circ}$ geringer als für reine Scherung (vergl. Abs. 2.2.2).

Die Anfangsrißlängen-Ausbreitungsgeschwindigkeits-Relation, wie oben beschrieben, gilt nicht für hochdynamische Vorgänge bei LECEI-Experimenten. Die während der Entwicklung des Spannungsintensitätsfaktors zu berücksichtigende effektive Rißlänge $a_{\text {eff }}$ ist das Produkt aus dem Belastungsintervall (vom Belastungsbeginn bis zur Rißinitiierung) und der Schallgeschwindigkeit des Materials. Diese effektive Rißlänge ist wesentlich geringer als die tatsächliche Ausdehnung des Starterkerbs. Vielmehr ist der maßgebliche Faktor zur Steuerung der Beanspruchungsrate gegeben durch die Projektilgeschwindigkeit zum Zeitpunkt des Aufpralls. Abbildung 32a zeigt, wie sich in zwei Experimenten (Probenmaterial: Stahl) mit unterschiedlichen Schlaggeschwin- 


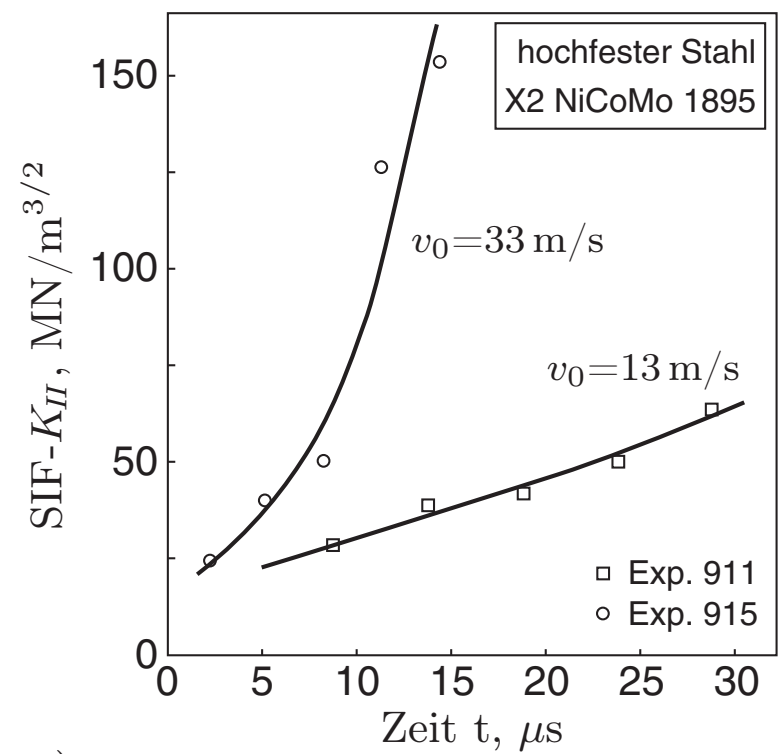

a)

Abb. 32: Abhängigkeit der Beanspruchungsrate $\dot{K}_{I I}$ von Schlaggeschwindigkeit $v_{0}$ und Materialeigenschaften (aus [148]).

digkeiten unterschiedliche Beanspruchungsraten $\dot{K}_{I I}$ entwickeln. Im Modell besteht zwischen der Schlaggeschwindigkeit und der Beanspruchungsrate ein linearer Zusammenhang (siehe Anhang E). Weiterhin ist das Ausbilden des Beanspruchungsfeldes an die Materialeigenschaften gebunden, wie der Vergleich der Experimente an Stahl (Abb.32a) mit einer Serie von Experimenten an dem Epoxidharz Araldit-B (Abb.32b)

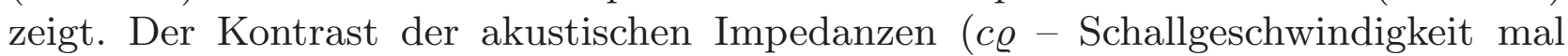
Dichte) zwischen Projektil und Probenmaterial bestimmt den Amplitudenanstieg des Spannungspulses innerhalb der Druckwelle und beeinflußt somit mittelbar ebenfalls die Beanspruchungsrate [150]. Die infolge des Auftreffens eines Stahlprojektils auf eine Gesteinsprobe resultierenden Partikelgeschwindigkeiten von ca. 0,8v0 sind um einen Betrag von ca. $30 \%$ der Stoßgeschwindigkeit $v_{0}$ höher als ohne Impedanzkontrast [151]. Eine Parametervariation bei der Finite-Elemente-Modellierung liefert dieselbe Erhöhung für den Spannungsintensitätsfaktor $K_{I I}$ (siehe Anhang E).

\subsection{Globalenergie-Verfahren}

Die mittels der, in den vorstehenden Abschnitten geschilderten, weiterführenden Methoden zur Kennwertbestimmung und zur Erzeugung schneller Rißausbreitung erzielten Ergebnisse werden mit einem standardisierten Testverfahren verifiziert. Hierzu werden Kerbschlagbiegeversuche an Proben des Charpy-Typs durchgeführt [152]. Bei dem eingesetzten instrumentierten Pendelschlagwerk (s. Abb. 33a) wird an der Hammerfinne während der mechanischen Beanspruchung der gekerbten Probe und dem anschließenden Versagen durch Brechen ein Last-Zeit-Signal $F(t)$ aufgenom- 


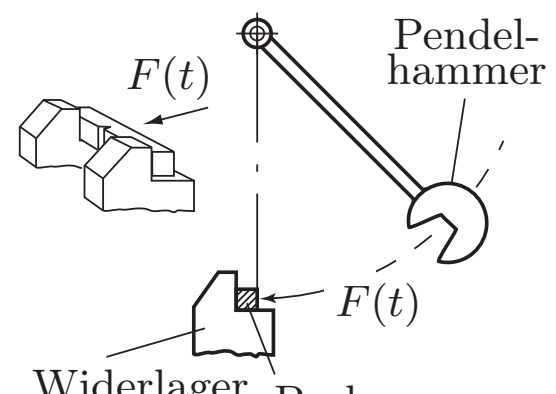

Widerlager Probe

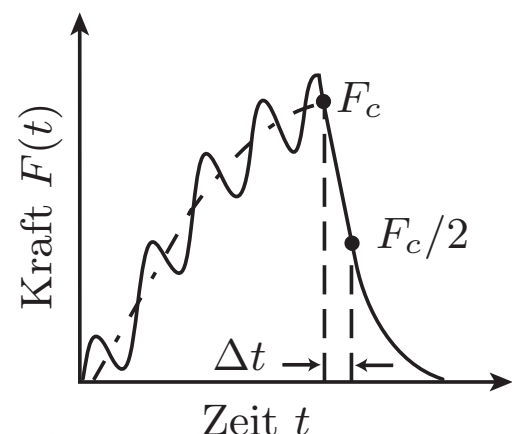

b)

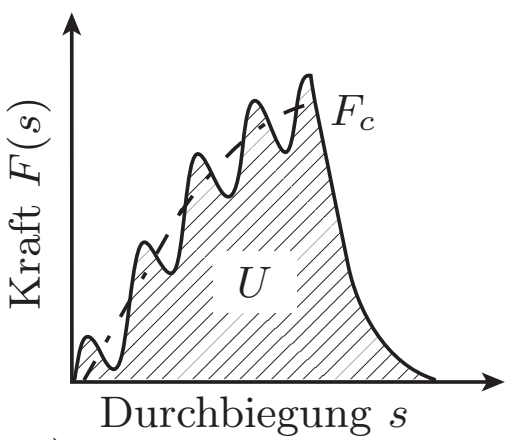

c)

a)

Abb. 33: Prinzip der Globaldaten-Ermittlung im Schlagbiegeversuch.

men (s. Abb. 33b). Die beim Bruch umgesetzte Gesamtenergie $U$ wird aus KraftDurchbiegungs-Kurven $F(s)$ (s. Abb. 33c) ermittelt:

$$
U=\int F(s) \mathrm{d} s .
$$

Die Durchbiegung $s$ ist gegeben durch:

$$
s(t)=\int\left(v_{0}-\frac{1}{m} \int F(t) \mathrm{d} t\right) \mathrm{d} t,
$$

wobei $v_{0}$ die Schlaggeschwindigkeit und $m$ die Masse des Pendelhammers bezeichnet.

Für Proben der Höhe $W$ mit unterschiedlichen Starterkerblängen $a_{0}$ und damit unterschiedlichen Rißlaufwegen $a_{L}=W-a_{0}$ werden aus $U\left(a_{L}\right)$ entsprechend der Definition der Größe $G$ (s. Gl. 11) Approximationen für die Rißlaufenergie $G_{I D}$ bestimmt. Für das jeweilige Rißlaufereignis wird aus dem Rißlaufweg und der Bruchdauer $2 \Delta t$ eine mittlere Rißlaufgeschwindigkeit abgeschätzt. Die so mittelnd über dem Geschwindigkeitsverlauf erzielten Ergebnisse werden als Kontrolldaten verwendet. Darüber hinaus wird aus der Bruchlast $F_{c}$, die durch eine Ausgleichskurve aus dem Lastsignal abgeleitet wird (Abb. 33b), nach dem ASTM-Normprüfverfahren (siehe auch [153]) die Bruchzähigkeit bei Initiierung $K_{I d}^{*}$ bestimmt.

\subsection{Bruchgeschwindigkeitsabschätzung mit Wallnerlinien}

Zur Bestimmung der Rißlaufgeschwindigkeit von Rißlaufereignissen in Einkristallen wird ein Verfahren eingesetzt, das auf einer Analyse der Bruchflächenmorphologie beruht [154-156]. Die Überlagerung von Schallwellen mit dem an der Rißspitze bzw. der Bruchfront mitbewegten Spannungsintensitätsfeld erzeugt Unebenheiten in der Bruchfläche, die als Wallnerlinien bezeichnet werden. Die Emission von Schallwellen erfolgt während des Bruches beispielsweise an Fehlstellen, die von der Bruchfront 
durchlaufen werden und an denen das Spannungsfeld infolge der Kerbwirkung Variationen unterliegt. Die Auswertung von Wallnerlinien auf Bruchflächen ergibt eine Abschätzung für die Bruchausbreitungsgeschwindigkeit im Verhältnis zur Scherwellengeschwindigkeit $v_{s}$. Die Scherwellengeschwindigkeit ist deshalb maßgeblich, weil nur transversale Wellen Spannungsschwankungen in geeigneter Richtung und Stärke verursachen. Eine schematische Darstellung der Entstehung von Wallnerlinien ist in Abb. 34 für die Ausbreitung einer geraden Bruchfront (Abb. 34a) und für eine gekrümmte Bruchfront (Abb. 34b) angegeben. Den Ursprung von Wallnerlinien (in der Abbildung sind die Fehlstellen mit q gekennzeichnet) bilden in Laborproben häufig Störquellen, die bei der Probenbearbeitung entstanden sind. Neben der Krümmung der Bruchfront ergeben sich nichtlineare Wallnerlinien auch, wenn sich die Bruchausbreitungsgeschwindigkeit $v_{c}$ während des Bruchvorganges ändert.

Um eine gute Abschätzung der Bruchausbreitungsgeschwindigkeit zu erhalten, empfiehlt es sich, wenn möglich, mehrere der im folgenden geschilderten Auswertemöglichkeiten $\mathrm{zu}$ kombinieren:

a) Bei der Ausbreitung von ebenen Bruchfronten entstehen lineare Wallnerlinien (Abb.34a). Diese schließen im Falle sich kreuzender Linien einen Winkel $\psi$ ein, d.h. sie bilden einen Winkel von $\psi / 2$ mit der Normalen auf der Bruchausbreitungsrichtung (Abb. 35a). Anhand einfacher Überlegungen ergibt sich (vergl. Abb. 35):

$$
v_{c}=v_{s} \cos \psi / 2
$$

b) finden sich gekrümmte Wallnerlinien, folgt eine Abschätzung der Bruchgeschwindigkeit aus dem Verhältnis der durch Abb. 35b beschriebenen Winkel:

$$
v_{c}=v_{s} \cos \alpha / \cos \beta
$$

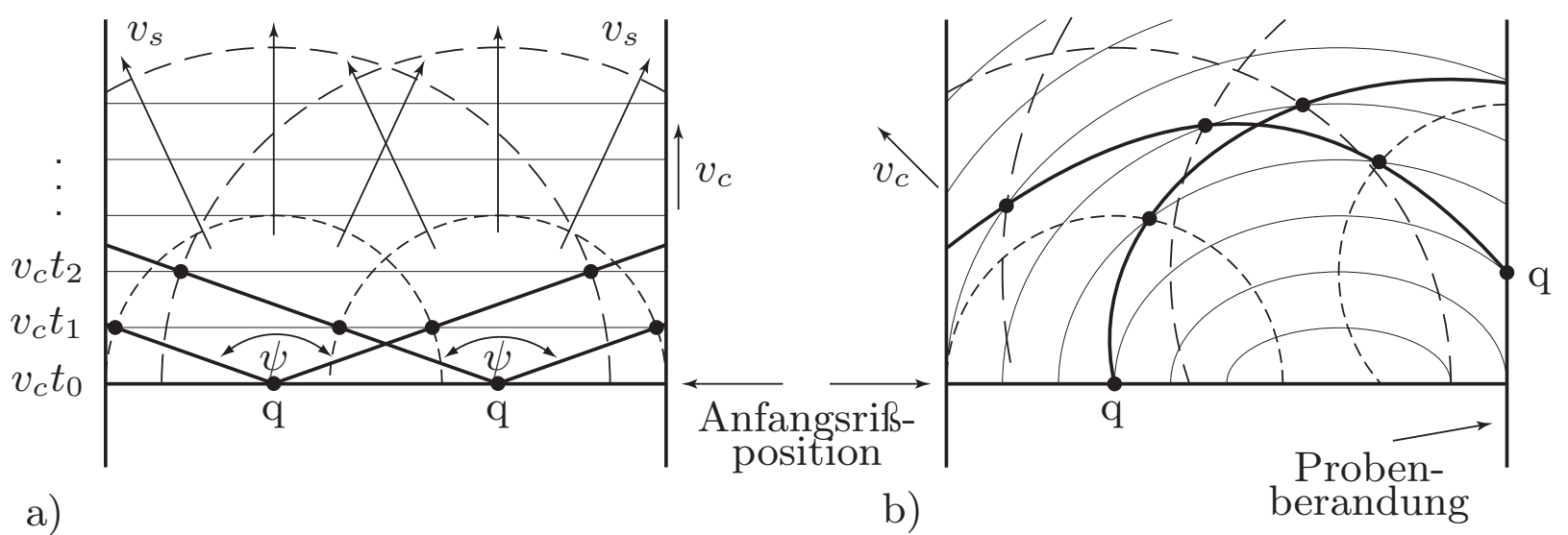

Abb. 34: Entstehung von Wallnerlinien: a) bei gerader Bruchfront und b) bei gekrümmter Bruchfront $\left(v_{s} / v_{c}=3\right)$. 


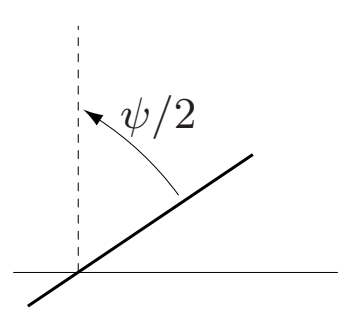

a)

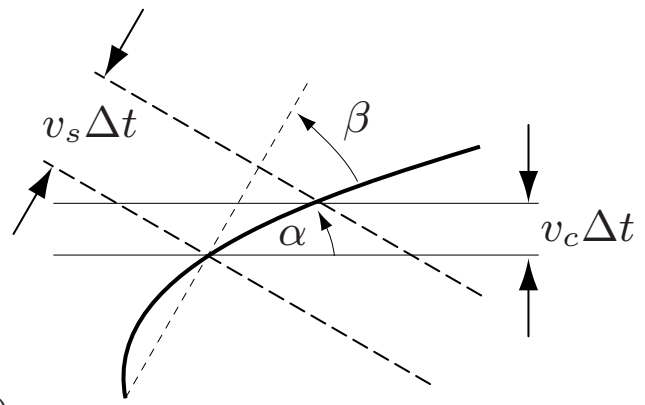

b)

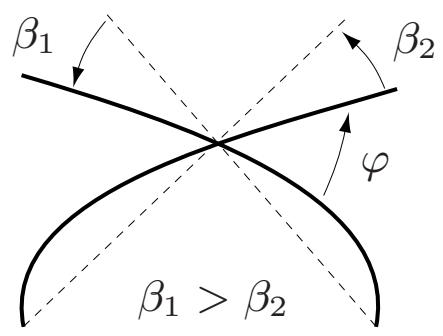

c)

Abb. 35: Auswertung von Wallnerlinien: a) anhand von linearen Wallnerlinien, b) aus einzelnen, gekrümmten Wallnerlinien und c) aus Paaren von kreuzenden Wallnerlinien.

c) bei sich kreuzenden, gekrümmten Linien (Abb. 35c) ist:

$$
v_{c}=v_{s} \sin \varphi /\left[\sin ^{2} \varphi \cos ^{2} \beta_{1}+\left(\cos \varphi \cos \beta_{1}+\cos \beta_{2}\right)^{2}\right]^{1 / 2} \text {. }
$$




\section{Ergebnisse}

\subsection{Probenmaterial und Probendimensionierung}

Es wurden Experimente an zwei verschiedenen Gesteinstypen durchgeführt, an Solnhofener Kalkstein und an Arkansas Novaculit, einem Quarzitgestein. Diese beiden Gesteine wurden bereits eingehend studiert [103,157-159], die bekannten Parameter (ergänzt und verifiziert durch eigene Messungen) sind:

Tabelle 1: Materialparameter der untersuchten Gesteine

\begin{tabular}{|l||c|c|c|c|c|c|}
\hline Gesteinstyp & $\begin{array}{c}\text { E-Modul, } \\
\text { GPa }\end{array}$ & $\begin{array}{c}\text { Poisson- } \\
\text { zahl }\end{array}$ & $\begin{array}{c}v_{p}, \\
\mathrm{~m} / \mathrm{s}\end{array}$ & $\begin{array}{c}v_{s}, \\
\mathrm{~m} / \mathrm{s}\end{array}$ & $\begin{array}{c}v_{R}, \\
\mathrm{~m} / \mathrm{s}\end{array}$ & $\begin{array}{c}\text { Dichte, } \\
\mathrm{kg} / \mathrm{m}^{3}\end{array}$ \\
\hline \hline Solnh. Kalkstein & 62 & 0,30 & 5700 & 3000 & 2800 & 2600 \\
Arkans. Novaculit & 85 & 0,17 & 5900 & 3700 & 3300 & 2650 \\
\hline
\end{tabular}

Anhand von Bruchzähigkeits- und Zugfestigkeitsdaten aus verschiedenen Quellen [103, 159-163] ergeben sich nach den Gln. (7) und (10), wie in der folgenden Tabelle angegeben, Abschätzungen für die zu erwartenden Prozeßzonengrößen und die darüber bestimmten Mindestprobendimensionen:

Tabelle 2: Literaturdaten zu Bruchzähigkeit und Zugfestigkeit der untersuchten Gesteine sowie daraus abgeleitete Prozeßzonen- und Mindestprobendimensionen; ergänzt durch Angaben zu Korndurchmesser und Porosität

\begin{tabular}{|l||c|c||c|c|c||c|c|}
\hline Gesteinstyp & $\begin{array}{c}K_{I c}, \\
\mathrm{MN} / \mathrm{m}^{\frac{3}{2}}\end{array}$ & $\begin{array}{c}\sigma_{t}, \\
\mathrm{MPa}\end{array}$ & $\begin{array}{c}r_{p}^{\mathrm{EDZ}} \\
\mathrm{mm}\end{array}$ & $\begin{array}{c}\left(\frac{K_{I c}}{\sigma_{t}}\right)^{2}, \\
\mathrm{~mm}\end{array}$ & $\begin{array}{c}d_{\text {min }}, \\
\mathrm{mm}\end{array}$ & $\begin{array}{c}\text { Korn- } \\
\text { größe, } \\
\mu \mathrm{m}\end{array}$ & $\begin{array}{c}\text { Poro- } \\
\text { sität, } \\
\%\end{array}$ \\
\hline \hline Solnh. Kalkstein & $0,8-1,1$ & 21 & 0,10 & 1,84 & 4,6 & $\leq 5$ & 4,0 \\
Arkans. Novaculit & $1,0-1,8$ & 45 & 0,05 & 0,83 & 2,1 & 10 & 0,1 \\
\hline
\end{tabular}

Bei der Festlegung der Probendimensionen waren jedoch weitere Faktoren zu berücksichtigen, um eine Beobachtung der Rißausbreitung über ausreichend lange Rißlaufwege zu ermöglichen. Dafür müssen zum einen im Drei-Punkt-Biege-Experiment (SENB-Proben) große Ligamentlängen in der Rißebene zur Verfügung stehen. Zum 
anderen kontrolliert in LECEI-Experimenten die Probengröße das Zeitfenster der, durch von der Probenberandung reflektierte Wellen unbeeinflußten, originären Beanspruchung. Folglich wurden, lediglich durch Beschränkungen in der Verfügbarkeit und den apparativen Aufbau begrenzt, sehr viel größere als die minimalen Probendimensionen festgelegt, so daß die Gültigkeit der in den Experimenten bestimmten Bruchzähigkeiten im Rahmen der Mindestprobenanforderungen gewährleistet ist (vergl. Abschnitt 2.1.4, Seite 12). Die verwendeten Proben unterteilen sich, nach der Verwendung der Initiierungsmethode, in jeweils drei Typen für jedes der untersuchten Gesteine:

Tabelle 3: Probendimensionen bezüglich Probenbreite $L$, -höhe $W$ und -dicke $B$ und Zuordnung der Probentypen zu der Belastungsmethode

\begin{tabular}{|c||c||c|l|}
\hline Gesteinstyp & Probentyp & $L \times W \times B, \mathrm{~mm}$ & Belastungsmethode \\
\hline \hline Soln- & Typ I & $600 \times 200 \times 20$ & quasistatisch \& Fallwerk \\
hofener & Typ II & $200 \times 200 \times 20$ & LECEI \\
Kalkstein & Typ III & $55 \times 10 \times 10$ & Global-Energie \\
\hline Ar- & Typ A & $306 \times 117 \times 22$ & quasistaisch \\
kansas & Typ B & $350 \times 204 \times 22$ & Fallwerk \\
Novaculit & Typ C & $204 \times 204 \times 22$ & LECEI \\
\hline
\end{tabular}

Durch die festgelegten Probengrößen ergeben sich für die Drei-Punkt-Biege-Proben (SENB), die unter Verwendung des Dehnungsmeßstreifen-Ketten-Verfahrens getestet wurden (Typ I bzw. Typ A und Typ B) große Ligamentlängen $W-a_{0}$. Die Starterkerben hatten eine Länge von bis zu $a_{0}=80 \mathrm{~mm}$ (Typ I) und von bis zu $a_{0}=40 \mathrm{~mm}$ (Typ A und Typ B). Die Dimensionen der LECEI-Proben (Typ II und Typ C) legen das Zeitfenster, zwischen Projektilaufschlag und Eintreffen der reflektierten Wellen an der Rißspitze, zu jeweils (d. h. für beide Gesteinstypen) mehr als $45 \mu$ s fest. Vom Beginn der Rißspitzenbeanspruchung als Zeitnullpunkt an (festgestellt durch den Rißspitzendehnungsmeßstreifen, s. u.), ergibt sich ein Zeitraum von jeweils $30 \mu$ s unbeeinflußter, originärer Beanspruchung (siehe Anhang D).

Die Probenpräparation erfolgte unter Einsatz geeigneter Diamantwerkzeuge. Die Maßstoleranz beträgt 1/10 mm oder besser. Besondere Sorgfalt herrschte bei der Herstellung der Initiierungskerben. Der Kerbradius für die Probentypen I und II sowie A-C wurde zwischen $0,5 \mathrm{~mm}$ und $1 \mathrm{~mm}$ variiert. Diese Kerben wurden mit einem Diamantsägeblatt mit elliptischem Querschnitt in die Proben eingebracht, teilweise wurde zur Erzeugung des Kerbgrundes zusätzlich ein Diamanthohlbohrer eingesetzt. Bei den Globaldatenexperimenten (Typ III) wurde eine Drahtsäge verwendet, auf deren Draht (Durchmesser 0,25 mm) eine Diamantschleifpaste aufgebracht wurde. 
Mechanische Veränderungen der Initiierungsstelle durch thermische Einflüsse während der Kerbgrunderzeugung können aufgrund der Verwendung von Wasserkühlung bzw. geringer Schleifgeschwindigkeiten weitgehend ausgeschlossen werden.

Bei beiden Gesteinen handelt es sich um monomineralische, polykristalline natürlich vorkommende Geomaterialien. Solnhofener Kalkstein besteht zu über $99 \%$ aus Calcit $\left(\mathrm{CaCO}_{3}\right.$ - Calciumkarbonatkristalle), bei mikritischen Korngrößen von 1$5 \mu \mathrm{m}[164,165]$. Arkansas Novaculit besteht demgegenüber mit einem ebenso hohen Reinheitsgrad aus Quarz ( $\mathrm{SiO}_{2}$ - Siliziumdioxidkristalle [166]), die mittlere Korngröße ist $10 \mu \mathrm{m}$. Solnhofener Kalkstein, wegen der Art des Vorkommens und seiner Verwendung auch als Solnhofener Plattenkalk oder Litographieschiefer bezeichnet, wird wegen seiner gleichmäßigen feinkörnigen Struktur im Steindruck eingesetzt, aber auch u.a. als Bodenbelag in der Bauindustrie verwendet. Arkansas Novaculit wird hauptsächlich als Schleifmaterial gehandelt - Quarz hat die Härte 7 in der 10-teiligen Härteskala nach Moos und ist damit das härteste der gesteinsbildenden Minerale -, in der vorliegenden Qualität (Handelsbezeichnung: Black Hard Arkansas Novaculite) eignet er sich zum Schärfen chirurgischer Instrumente. Diese Gesteinstypen wurden als Untersuchungsmaterial ausgewählt, da sie hinsichtlich ihrer, gegenüber anderen Gesteinen, herausragenden Eigenschaften besonders gut für die geplanten Experimente einzusetzen sind. Sowohl für Solnhofener Kalkstein als auch für Arkansas Novaculit sind die resultierenden Prozeßzonen vergleichsweise klein. Dadurch ist das Konzept der linear elastischen Bruchmechanik auf diese Gesteine anwendbar. Beide Materialien eignen sich darüber hinaus wegen der geringen Korngrößen bzw. der ausgezeichneten Homogenität zum Einsatz mit der zur Spannungsbestimmung avisierten Dehnungsmeßstreifenmethode. Vorexperimente an Granit und Marmor haben gezeigt (siehe Anhang A), daß sich bei anderen Gesteinen, vermutlich in Abhängigkeit der Korngröße [167], trotz der sehr ähnlichen sonstigen Materialeigenschaften wesentlich ausgedehntere Prozeßzonen bilden können, als in den untersuchten Materialien, wodurch die experimentelle Umsetzung bei der Datengewinnung erschwert würde.

\subsection{Erzeugung von Rißlaufereignissen in Gestein}

Die Bruchausbreitung in sprödbrechendem Gestein weist eine Besonderheit auf. Abbildung 36 zeigt ein Vergleichsexperiment, das diese Besonderheit verdeutlicht. Dazu wurde in einem konventionellem, sprödbrechenden Modellmaterial, dem Epoxidharz Araldit B, unter quasistatischer Belastung und identischen Versuchsbedingungen bezüglich der Probendimensionen, der Anfangskerblänge sowie des Kerbradius und in Solnhofener Kalkstein Rißlaufereignisse initiiert. Bei dem Epoxidharz (Abb. 36a) tritt bereits nach einem kurzen Rißlaufweg Rißgabelung auf. Die Rißgabelung ist ein Zeichen dafür, daß der Riß die maximale Rißgeschwindigkeit erreicht hat (s. Abschnitt 2.3.4). Dies ist ein für sprödbrechende Materialien typisches Rißausbreitungsverhalten [8]. Demgegenüber läuft ein Riß in Solnhofener Kalkstein (Abb.36b) wäh- 

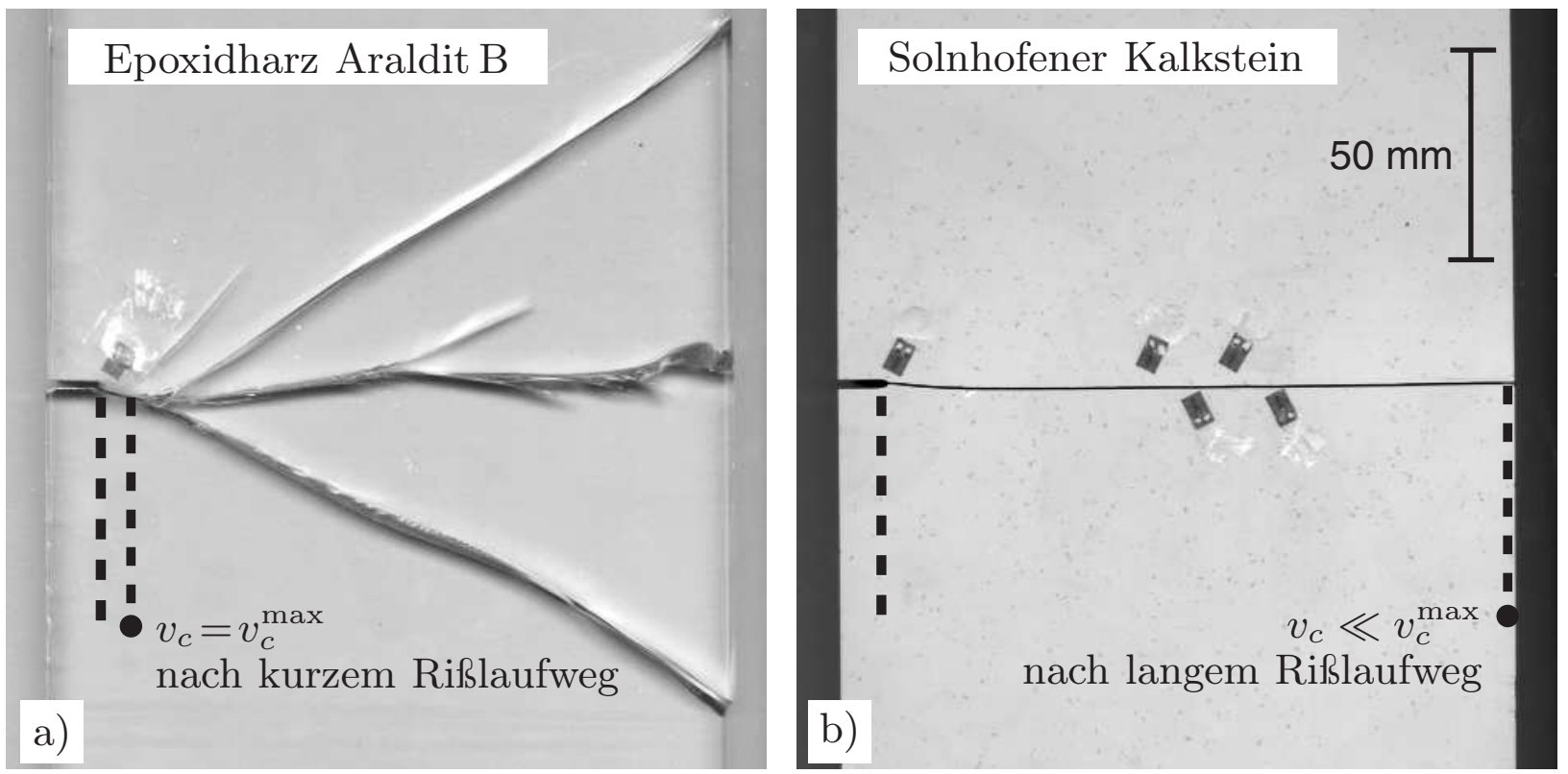

Abb. 36: Unterschied bei der Bruchausbreitung sprödbrechender Materialien: a) Epoxidharz Araldit-B zeigt Rißgabelung und b) Solnhofener Kalkstein Einzelrißausbreitung.

rend des gesamten Experiments, d.h. durch die gesamte Probe, als Einzelriß. Der Riß breitet sich somit über lange Rißlaufwege aus ohne seine maximale Rißlaufgeschwindigkeit zu erreichen. Dieses Rißausbreitungsverhalten ist typisch für Gesteine und wird nicht nur in den untersuchten Gesteinstypen Solnhofener Kalkstein und Arkansas Novaculit sondern auch z. B. in Marmor und Granit beobachtet. Es unterscheidet das Bruchausbreitungsverhalten in Gesteinen von dem klassischen Sprödbruch und steht in Einklang mit Ergebnissen früherer Studien [102,168]. Für sprödbrechende Gesteine ist demnach die Rißlaufenergie offensichtlich deutlich größer als die Rißinitiierungsenergie, so daß für Gesteine sehr große Rißlaufwege in Proben mit notgedrungenermaßen sehr großen Abmessungen erforderlich sind, damit sich an der Spitze des Risses Energien bzw. Spannungsintensitätsfaktoren hinreichender Größe einstellen und damit hohe Bruchgeschwindigkeiten erreichen lassen. Andererseits läßt sich ein, für schnelle Rißausbreitung erforderliches, erhöhtes Energieniveau auch, wie in dieser Arbeit geschehen, durch dynamische Initiierungsmethoden (siehe Abs. 3.3) in Schlag-/Stoßexperimenten und durch die Verwendung von Scher(ModusII)-Belastungsbedingungen realisieren.

Solche Schlag-/Stoßexperimente zur bruchmechanischen Kennwertbestimmung wurden im Rahmen dieser Arbeit erstmalig an Gesteinen durchgeführt. Dazu stand eine Fallgewichtsapparatur (Eigenkonstruktion) zur Verfügung. Die maximale Schlaggeschwindigkeit der Fallgewichtsapparatur beträgt $v_{0}=5 \mathrm{~m} / \mathrm{s}$, die Fallgewichtsmasse $8,1 \mathrm{~kg}$, mit der maximalen Fallhöhe von 1,3 m ergibt sich daraus ein maximales Ener- 
gieangebot von $100 \mathrm{~J}$. In der verwendeten LECEI-Testvorrichtung (Eigenkonstruktion) sind Projektilgeschwindigkeiten von $v_{0}=5-100 \mathrm{~m} / \mathrm{s}$ möglich, der maximale Autoklavendruck beträgt $10 \mathrm{MPa}$, die Länge des Beschleunigungsrohres $3 \mathrm{~m}$ (siehe [138]); die Spezifikationen des eingesetzten Projektils aus gehärtetem C60 Stahl sind: Länge $150 \mathrm{~mm}$, Querschnitt $50 \mathrm{~mm}$, Masse 2,3 kg, wodurch ein maximales Energieangebot von 11,5 kJ resultiert. Zur Erzeugung langsamer Rißlaufereignisse wurde eine elektronisch geregelte, programmierbare servo-hydraulische Universalprüfmaschine (Typ INSTRON) mit folgenden Spezifikationen eingesetzt: $50 \mathrm{kN}$ maximale Last, $1 \mathrm{kHz}$ Regelrate, Säulen zusätzlich versteift, übliche Kolbengeschwindigkeit $v_{0}=10^{-6}$ $10^{-5} \mathrm{~m} / \mathrm{s}$. Zum Einsatz servo-hydraulischer Belastungsapparaturen in der Gesteinsmechanik siehe [168-170]. Die Signale instrumentierter Finnen in dem letztgenannten Versuchsstand ermöglichten es in jedem Experiment zu Kontroll- und Kalibrierungszwecken aus den, direkt an der Einleitungsstelle gemessenen Lasten, vergleichend Initiierungsbruchzähigkeiten zu ermitteln (siehe Anhang C).

\subsection{Experimentell in Gestein erzielte Bruchgeschwindigkeiten}

Als Maß für die erreichten Bruchlaufgeschwindigkeiten sind in Abb. 37 Ausschnitte von Bruchflächen aus drei Experimenten in Solnhofener Kalkstein angegeben, die

a)

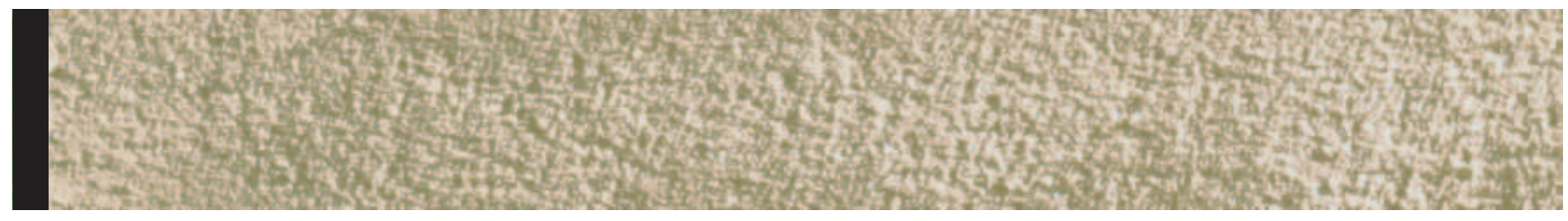

b)

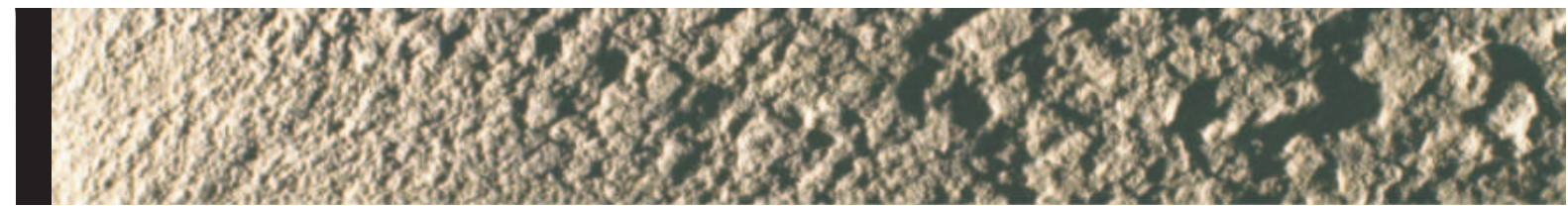

c)

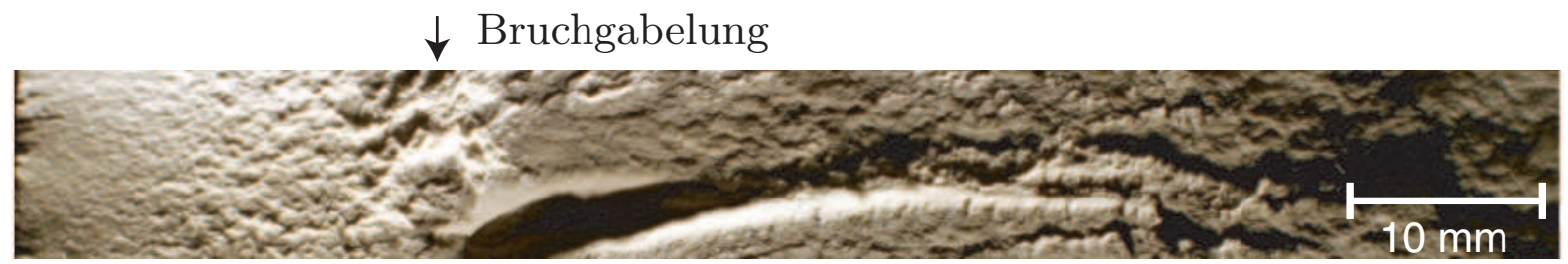

Abb. 37: Typische Bruchflächen in Solnhofener Kalkstein mit a) quasistatischer Initiierung sowie dynamisch initiiert im b) Fallwerk- und c) LECEI-Experiment (Bruchausbreitung jeweils von links nach rechts, die Initiierungskerben befinden sich am linken Bildrand). 
mit den vorstehend genannten Initiierungsmethoden durchgeführt wurden. Der Unterschied der mit den verschiedenen Methoden erreichten Geschwindigkeitsniveaus drückt sich in der Entwicklung der Oberflächenrauhigkeit auf den Bruchflächen aus. Die Bruchflächenrauhigkeit ist ein Maß für die bei dem Bruchvorgang dissipierte Energie [171-173]. Eine zunehmende Bruchflächenrauhigkeit entspricht einem steigenden Bruchwiderstand und somit steigenden Rißlaufzähigkeiten [91]. Dabei ist vereinfacht ausgedrückt - die Morphologie der Bruchfläche proportional der bei ihrer Erzeugung dissipierten Bruchenergie.

Ein Vergleich der Bruchfächen aus Abb. 37 zeigt deutliche Unterschiede in der Rauhigkeitsentwicklung der Bruchflächen. In den Experimenten mit quasistatischer Initiierung wurde in keinem der Experimente eine Zunahme der Bruchflächenrauhigkeit beobachtet. Das vorliegende Beispiel (Abb. 37a) zeigt ein Rißlaufereignis mit einer Bruchgeschwindigkeit von $v_{c}<10 \mathrm{~mm} / \mathrm{s}$. Insgesamt wurden mit dieser Initiierungsmethode, in Abhängigkeit der Anfangsrißlänge, Bruchgegeschwindigkeiten von bis zu $600 \mathrm{~m} / \mathrm{s}$ erzielt. Diese Bruchgegeschwindigkeiten liegen deutlich unter der maximalen Bruchgeschwindigkeit $v_{c}^{\max }$ (vergl. Abs. 2.3.4); sie entsprechen etwa $20 \%$ der Ausbreitungsgeschwindigkeit von Rayleigh-Wellen in diesen Materialien (siehe Tabelle 1).

Im Gegensatz dazu ist bei den dynamisch initiierten Bruchvorgängen (Abb. 37b und Abb. 37c) ein Anstieg der Rauhigkeit der Bruchfäche mit Fortschreiten des Risses zu erkennen. Dabei kann, trotz der raschen Geschwindigkeitszunahme in den unter Zug(Modus-I)-Initiierungsbedingungen durchgeführten Fallwerk-Experimenten, die maximale Bruchgeschwindigkeit $v_{c}^{\max }$ bei den verwendeten Probengrößen (Typ I bzw. Typ B aus Tabelle 3) nicht ganz erreicht werden. Die mit dieser Initiierungsmethode erzielten Bruchausbreitungsgeschwindigkeiten von maximal $1800 \mathrm{~m} / \mathrm{s}$ in Solnhofener Kalkstein (siehe Beispiel in Abb. 37b) und 1900 m/s in Arkansas Novaculit entsprechen ca. $60 \%$ der jeweiligen Rayleigh-Wellengeschwindigkeit. Eine weitere Steigerung der Bruchgeschwindigkeit mit dieser Rißinitiierungsmethode ist nicht möglich, da die Belastung aufgrund der linienhaften Lasteinleitung durch die Verwendung einer spitzen Belastungsfinne und die dadurch auftretenden Spannungskonzentrationen bei einer Erhöhung der Schlaggeschwindigkeit kritisch wird. Die Spannungskonzentrationen führen dazu, daß an der Lasteinleitungsstelle Materialversagen auftritt, wodurch die dort umgesetzte Energie für den Rißausbreitungsvorgang nicht zur Verfügung steht und die Anregung von elastischen Belastungswellen gestört wird. Die Schlaggeschwindigkeiten sollten somit im verwendeten experimentellen Aufbau auf $v_{0} \leq 3 \mathrm{~m} / \mathrm{s}$ beschränkt werden, bei gleichzeitiger Reduktion des maximal zur Verfügung stehenden Energieangebotes auf 36,5 J. Hierbei gibt die so durch die Schlag-/Stoßapparatur zur Verfügung gestellte potentielle Energie lediglich einen Rahmenwert an, da große Teile dieser Energie nicht beim Bruchvorgang umgesetzt werden, sondern bei der Beschleunigung der Probenhälften.

Die Bruchhöchstgeschwindigkeit $v_{c}^{\max }$ wird schließlich in den LECEI-Experimenten unter dynamischen Scher(Modus-II)-Initiierungsbedingungen erreicht. Die Verwen- 
dung höherenergetischer Methoden zur Rißinitiierung, etwa durch Explosionsbeanspruchung, oder der Einsatz größerer Proben mit längeren Rißlaufwegen ist somit nicht erforderlich. Als Bruchhöchstgeschwindigkeiten wurden in Solnhofener Kalkstein rund $2000 \mathrm{~m} / \mathrm{s}$ und in Arkansas Novaculit $2400 \mathrm{~m} / \mathrm{s}$ bestimmt. Dies entspricht in beiden Materialien etwa $70 \%$ der jeweiligen Rayleigh-Wellengeschwindigkeit. Bei Erreichen der maximalen Bruchgeschwindigkeit ist Rißgabelung zu beobachten. Abbildung 37c zeigt ein Beispiel, bei dem der Riß nach einem sehr raschen Geschwindigkeitsanstieg nach etwa $20 \mathrm{~mm}$ gabelt. Bei der Durchführung der LECEI-Experimente wurde der Rißausbreitungsvorgang mit der Cranz-SchardinHochgeschwindigkeitskamera (siehe Abs. 3.2.2) aufgenommen. In Abb. 38 sind solche Hochgeschwindigkeitsphotos dargestellt. Abbildung 38a zeigt eine Serie von Photos, die während des in Abb. 37c abgebildeten Experimentes aufgenommen wurden. Im Vergleich zu einem Experiment in Arkansas Novaculit (Abb. 38b) ist zu erkennen, daß der Riß in Solnhofener Kalkstein (Abb. 38a) nach einem größeren Rißlaufweg gabelt, obwohl die Stoßgeschwindigkeit hier mit ca. $v_{0}=15 \mathrm{~m} / \mathrm{s}$ gegenüber ca. $v_{0}=10 \mathrm{~m} / \mathrm{s}$ etwa um den Faktor 1/3 größer war. Da Risse in Arkansas Novaculit offensichtlich schneller beschleunigen als bei Solnhofener Kalkstein wurden, um die Rißlaufereignisse über einen ausreichenden Rißlaufweg zu beobachten, bei Arkansas Novaculit mit $v_{0}=7,5-10 \mathrm{~m} / \mathrm{s}$ geringere Stoßgeschwindigkeiten gegenüber $v_{0}=15$ $25 \mathrm{~m} / \mathrm{s}$ bei Solnhofener Kalkstein eingesetzt. Die Abknickwinkel, die sich zwischen den unter Scher(Modus-II)-Bedingungen belasteten Anfangskerben und den davon initiierten Zug(Modus-I)-Rissen einstellten, lagen im Mittel mit $67^{\circ}$ bei Solnhofener Kalkstein und mit $68^{\circ}$ bei Arkansas Novaculit geringfügig unter den $70,5^{\circ}$, die bei reiner Scherung auftreten würden. Dies ist in Übereinstimmung mit den Ergebnissen der Finite-Elemente-Modellierung (siehe Abs. 3.3.4). Materialversagen an der Lasteinleitungsstelle tritt in den LECEI-Experimenten wegen der Verteilung der Last auf die gesamte Kontaktläche zwischen Probe und Projektil nicht auf. Daher lassen sich hier höhere Belastungsgeschwindigkeiten realisieren. Der vollständige Ablauf ei-
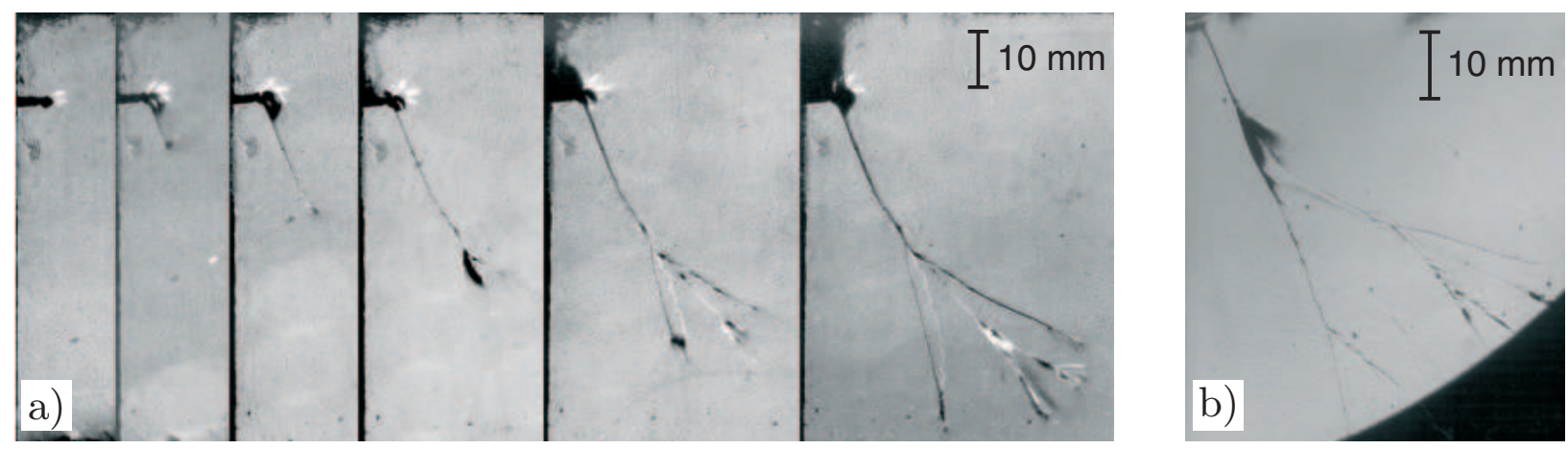

Abb. 38: Hochgeschwindigkeitsphotos von Rißlaufereignissen mit Bruchgabelung, initiiert im LECEI-Experiment: a) in Solnhofener Kalkstein (Bildfolge $8 \mu \mathrm{s}$ ) und b) Arkansas Novaculit. 
nes solchen Experiments ist in Anhang D beschrieben. Nach der Rißgabelung pflanzt sich der Riß mit der gleichen, konstanten Rißlaufgeschwindigkeit, der Bruchhöchstgeschwindigkeit $v_{c}^{\max }$, fort und gabelt dabei mehrfach. Die Bruchfächenrauhigkeit ist hierbei konstant. Da der Riß sich jedoch auf zwei oder mehr Bruchflächen gleichzeitig ausbreitet, steigt die insgesamt dissipierte Energie weiter an.

Die Abwesenheit von Scherlippen, wie sie häufig in Bruchexperimenten an metallischen Materialien auftreten [174], unabhängig von Initiierungsmethode und Geschwindigkeitsniveau verdeutlicht den spröden Charakter von Bruchvorgängen in Gesteinen. Es sei darauf verwiesen, daß die erzielten Ergebnisse grundsätzlich auf andere Gesteine übertragbar sind bzw. prinzipiell die gleichen Ergebnisse erzielt werden. So wurde beispielsweise Bruchgabelung auch an Marmor beobachtet, allerdings erst nach vergleichsweise größeren Rißlaufwegen. Lediglich die Meßtechnik wäre durch die geringere Homogenität, die größeren Korngrößen und die größere Prozeßzonenausdehnung erschwert. Es müßten größere Dehnungsmeßstreifen verwendet, größere Distanzen der Dehnungsmeßstreifen zum Riß eingehalten und größere Proben eingesetzt werden. Dies würde auch die zeitliche Signalauflösung sowie die Signalamplituden negativ beeinflussen.

\subsection{Meßdatengewinnung}

\subsubsection{Vorbemerkungen}

Mit dem in Abschnitt 3.2 beschriebenen Dehnungsmeßstreifen-Ketten-Verfahren wurden Spannungsintensitäten bzw. Bruchzähigkeiten während der Ausbreitung von Rissen in den o. a. Gesteinen bestimmt. Bei den Messungen wurden Dehnungsmeßstreifen des Typs 3/120LY41 (Hersteller HBM, Fläche $3 \times 1$ mm, $120 \Omega$ Grundwiderstand) unter Verwendung einer Wheatstone 1/4-Brückenschaltung in einer Entfernung von $5 \mathrm{~mm}$ zur Rißspitze appliziert. Die Signale wurden mit Signalträger-Meßverstärkern (Eigenkonstruktion, mit Hardware-Tiefpassfilter, $1 \mathrm{MHz}$ obere Abschneidefrequenz) 100-fach verstärkt und mit digitalen Speicheroszilloskopen (Yokogawa, Typ DL708E und DL716E: max. Speichertiefe 10 M-Worte, 12 bit Amplitudendynamik) aufgezeichnet. Als optimale Versorgungsspannung wurde nach Langzeitexperimenten an den untersuchten Gesteinen ein Wert von $6 \mathrm{~V}$ bestimmt. Die Meßdaten wurden zur Verbesserung des Signal-Rausch-Verhältnisses zur Darstellung und Auswertung ggf. mit Butterworth-Tiefpassfiltern unter Berücksichtigung der Signalfrequenz und Kontrolle der Amplitudentreue nachbearbeitet. Amplitudentreue ist gegeben, wenn bei Tiefpassfiltern die Filterfrequenz mindestens zehn mal größer als der Quotient aus Signalund Nyquistfrequenz ist. Die so gewonnenen Rißlaufzähigkeitsdaten wurden, wie in Abschnitt 3.2.2 beschrieben, den gegenwärtigen Ausbreitungsgeschwindigkeiten zugeordnet. Insgesamt wurden 53 Hauptexperimente durchgeführt und 142 Datenpunkte gewonnen. Pro Experiment wurden in der Regel sechs Dehnungsmeßstreifen auf der 
Probe appliziert. Je nach Art der Applikation - d. h. Instrumentierung eines Bereiches des Rißlaufereignisses mit konstanter oder steigender Rißlaufgeschwindigkeit wurde durch Mittelung mehrerer Ergebnisse ein einzelner Rißlaufzähigkeitswert oder durch individuelle Auswertung der Registrierungen mehrere Rißlaufzähigkeitswerte pro Probe ermittelt. Ergebnisse zur Rißlaufzähigkeitsmessung und die wesentlichen Parametervariationen werden im folgenden für die unterschiedlichen Phasen der Rißausbreitung anhand einiger Beispiele erläutert.

\subsubsection{Dehnungsmeßstreifensignale zur Rißlaufzähigkeitsmessung an Gestein}

Mit dem Dehnungsmeßstreifen-Ketten-Verfahren (Abschnitt 3.2) wurden Spannungsintensitäten/Bruchzähigkeiten an propagierenden Rissen bei der gegenwärtigen Ausbreitungsgeschwindigkeit bestimmt. Meßergebnisse für die Rißinitiierung und den Beginn der Rißausbreitung sind in Abb. 39angegeben. In dieser, wie auch in den folgenden Abbildungen, ist der Abstand $\Delta x$ der Dehnungsmeßstreifen in Ausbreitungsrichtung angegeben als $\Delta x_{01}$ für die Distanz zwischen DMS 0 und DMS 1 usw. Des weiteren werden die experimentellen Rahmendaten bezüglich der Belastungsart und der Probenform aufgeführt. Das Beispiel in Abb. 39 zeigt Daten eines quasistatisch initiierten Rißlaufexperimentes in Solnhofener Kalkstein. Es ist zu beobachten, daß die Spannungsintensität an dem Dehnungsmeßstreifen (DMS0), der zur Kon-

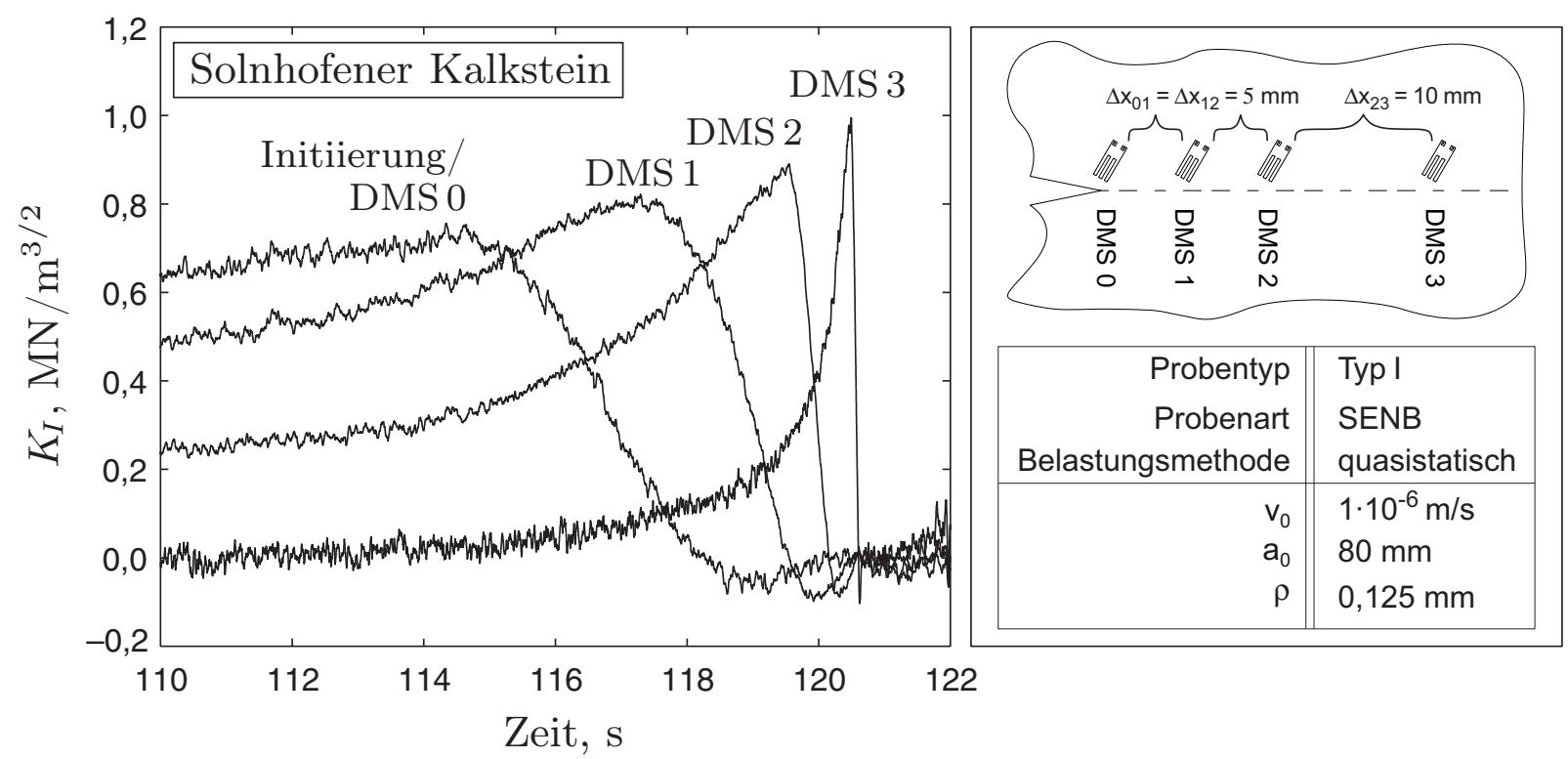

Abb. 39: Dehnungsmeßstreifen-Signale für Solnhofener Kalkstein (Probe KD01): Initiierung und Rißlaufbeginn; unter quasistatischen Belastungsbedingungen erzeugt. 
trolle des Initiierungsvorganges in der Nähe der Rißspitze appliziert wurde, infolge der mit einer konstanten Kolbengeschwindigkeit $\left(v_{0}=10^{-6} \mathrm{~m} / \mathrm{s}\right)$ aufgebrachten Belastung zunächst kontinuierlich ansteigt. Die Rißausbreitung beginnt nachdem der kritische Wert der Spannungsintensität erreicht ist. Sobald der Riß initiiert ist und sich damit der an die Rißspitze gebundene Spannungskonzentrationspunkt von dem Dehnungsmeßstreifen entfernt, fällt das Signal ab. Während des Rißausbreitungsprozesses passiert die Rißspitze nacheinander die entlang des Rißpfades applizierten Dehnungsmeßstreifen (DMS 1-3). Dabei steigt für jeden einzelnen der Dehnungsmeßstreifen die aus dem Dehnungsmeßstreifensignal bestimmte Spannungsintensität bei der Annäherung der Rißspitze an. Nachdem die Rißspitze die einzelnen Dehnungsmeßstreifen passiert hat, fällt das jeweilige Signal ab. Das Maximum der Meßkurven legt die Rißlaufzähigkeit fest. Die Rißlaufgeschwindigkeit bestimmt die Signalform, bzw. den Signalabstand. Aus der Signalcharakteristik im abgebildeten Beispiel ist zu ersehen (am deutlichsten an der Verminderung der Halbwertsbreite der Signale zu erkennen), daß die Rißausbreitung beschleunigt verläuft. Dabei steigt mit zunehmender Rißlaufgeschwindigkeit auch, bereits direkt nach der Initiierung, die Rißlaufzähigkeit an. Der Ursprung der Zeitachse wurde hier, wie auch in allen folgenden Beispielen, auf den Beginn der Rißspitzenbeanspruchung gelegt.

Bei Arkansas Novaculit bestimmt unter den angegebenen Versuchsbedingungen ein sogennantes „pop-in“-Verhalten den Anfangsbereich der Rißausbreitung (Abb. 40). Dabei breitet sich der Riß im Anschluß an die Initiierung für einen begrenzten Zeitraum sprunghaft aus. Dieses Verhalten ist in Abb. 40a an dem initialen Steilabfall des Signals des Rißspitzendehnungsmeßstreifens (DMS0) nach der linearen Belastungsphase zu erkennen. Anders als in Solnhofener Kalkstein wird offensichtlich das sich während der quasistatischen Beanspruchung einstellende Überangebot an Spannungsintensität (experimentell realisiert durch Vorgabe bestimmter Rißlängen $a_{0}$ bzw. Belastungskolbengeschwindigkeiten $v_{0}$ und die Verwendung großer Kerbradien $\rho$, s. Abs. 3.3.1) in Arkansas Novaculit abgebaut, indem sich eine metastabile Rißausbreitung einstellt (siehe z. B. [4]). Danach verflacht die Signalsteigung, der Riß breitet sich zunächst stabil und subkritisch aus. Während dieser Phase wird an der Rißspitze durch die mit konstanter Kolbengeschwindigkeit $\left(v_{0}=10^{-5} \mathrm{~m} / \mathrm{s}\right)$ fortgesetzte Belastung langsam wieder ein Spannungsintensitätsfeld aufgebaut, aufgrund dessen die Signalsteigung graduell zunimmt. Das Signal des Rißspitzendehnungsmeßstreifens (DMS 0) weist dabei eine zunehmend fallende, die folgenden Dehnungsmeßstreifen (DMS 1 und DMS 2) eine zunehmend steigende Charakteristik auf. Infolge dieser sekundären Belastungsphase initiiert der Riß erneut, diesmal von der scharfen Rißspitze, die während dieses Prozesses entstanden ist. Diese Initiierungsphase ist in Abb. 40b festgehalten. Man erkennt, daß das Signal des 1. Dehnungsmeßstreifens der Dehnungsmeßstreifenkette sein Maximum (in der Abbildung nicht erfasst: $K_{I, \mathrm{DMS} 1}^{\max }=2,75 \mathrm{MN} / \mathrm{m}^{3 / 2}$ ) bereits durchschritten hat und infolge der langsamen Rißausbreitung mit einer geringen Steigung abfällt. Dann, bei der erneuten Initiierung, nimmt die Steigung des Signalabfalls plötzlich zu. Das gleiche gilt für das, ebenfalls 

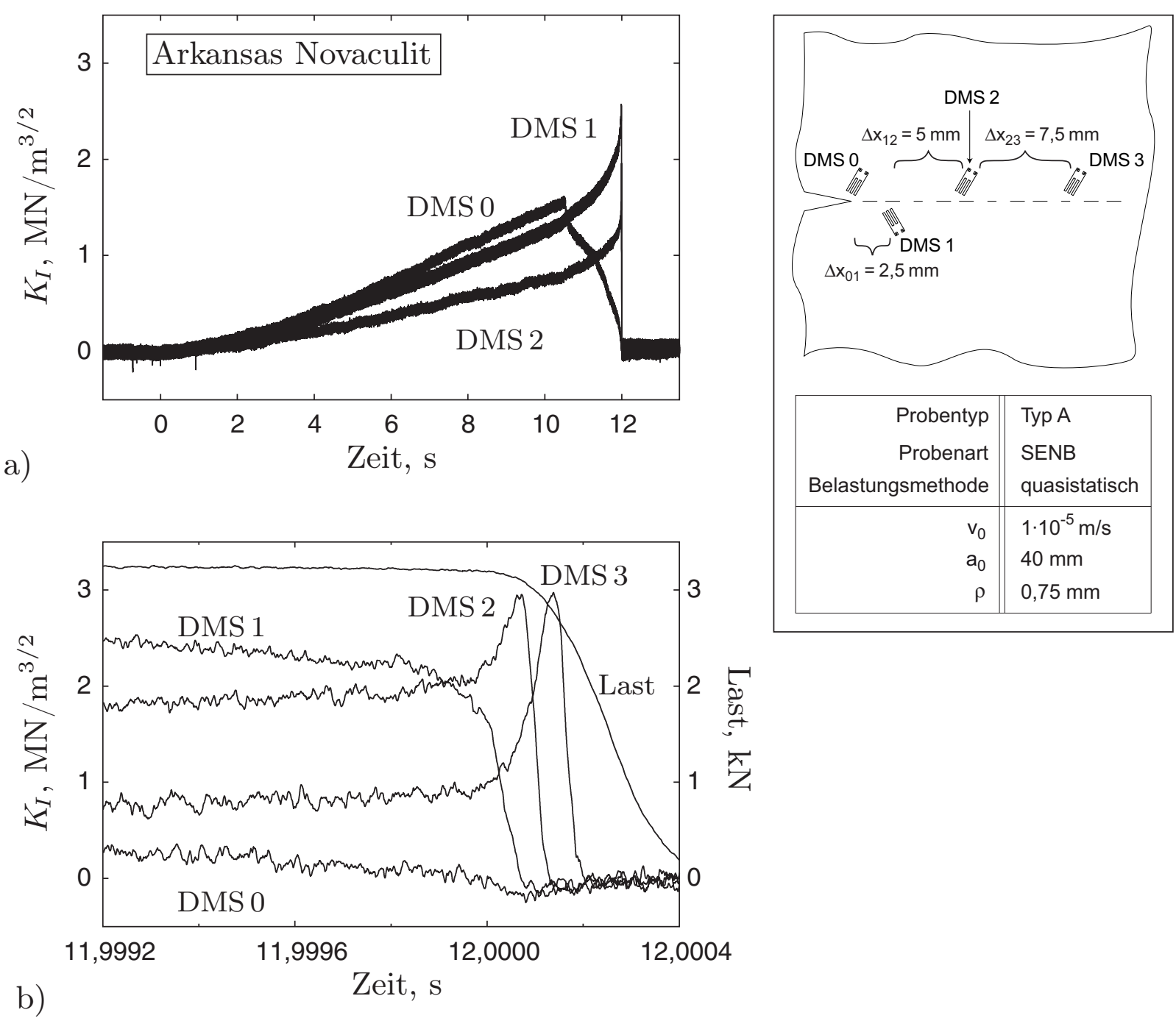

Abb. 40: Mit quasistatischer Belastung initiiertes Rißlaufereignis in Arkansas Novaculit (Probe NQC1). Die Dehnungsmeßstreifen-Signale zeigen: a) das gesamte Experiment mit Belastungsvorgang, Initiierungsphasen und Rißlaufprozeß und b) den Zeitabschnitt mit Beginn und Verlauf der überkritischen Rißausbreitung.

in der Abbildung angegebene, mit der Belastungsfinne an der Lasteinleitungsstelle aufgenommene Lastsignal. Wird das Lastsignal mittels der durch die Dehnungsmeßstreifen bestimmte Rißspitzenposition zum Zeitpunkt der sekundären Initiierung ausgewertet, ergibt sich mit $K_{I c}=2,8 \mathrm{MN} / \mathrm{m}^{3 / 2}$ ein Wert, der deutlich über dem der primären Initiierung liegt (vergl. Abb. 40a). Daraufhin erreicht der Riß schnell eine Rißlaufgeschwindigkeit von $v_{c}>1 \mathrm{~m} / \mathrm{s}$ und breitet sich überkritisch aus. Zwischen der ersten und der zweiten Initiierung liegt im angegebenen Beispiel ein Zeitraum von etwa 1,5 s. Für die Registrierung des Belastungsvorganges einschließlich der Initiierung und des Rißausbreitungsvorganges werden bei quasistatischer Initiierung zwei Datenerfassungen mit unterschiedlichen Abtastraten eingesetzt (dies gilt ebenfalls für 
Experimente an Solnhofener Kalkstein). Die Maxima, die sich bei der schnellen Rißausbreitung ergeben, werden bei der geringen Abtastrate, die für die Aufzeichnung des Belastungsvorganges notwendig ist, nicht erfaßst.

Während die Rißausbreitung in den in einer Drei-Punkt-Biege-Anordnung getesteten Proben (SENB) zu Beginn beschleunigt verläuft, stellt sich im Zentrum der Proben aufgrund der sich dem initial dominierenden Zugspannungsfeld überlagernden Druckspannungen, die von der Lasteinleitungsstelle ausgehen, eine konstante Rißausbreitungsgeschwindigkeit ein. Dies ist in Abb. 41 für ein weiteres, unter quasistatischer Initiierung an Solnhofener Kalkstein durchgeführtes Rißlaufexperiment gezeigt. Bei diesem Experiment wurden drei Dehnungsmeßstreifen der Dehnungsmeßstreifenkette in enger Folge im Probenzentrum appliziert. Es ist zu erkennen, daß die Rißausbreitungsgeschwindigkeiten noch ansteigt, nachdem der erste Dehnungsmeßstreifen passiert wurde, danach aber annähernd konstant ist (dabei ist die Applikationsdistanz der drei letzten Dehnungsmeßstreifen geringer, als die zum ersten Dehnungsmeßstreifen). Ein Vergleich der für Solnhofener Kalkstein (Abb. 39 und Abb.41) und für Arkansas Novaculit (Abb.40) abgebildeten Beispiele zeigt, daß das Signalminimum, das sich für Solnhofener Kalkstein einstellt, deutlicher ausgeprägt ist. Ursächlich für diesen Effekt ist die vergleichsweise höhere Poissonzahl dieses Gesteinsmaterials gegenüber Arkansas Novaculit (siehe Abs. 3.2, auch Anhang B).

Die angeführten Beispiele für quasistatische Rißinitiierung in Gestein zeigen, daß sich während des Rißausbreitungsprozesses deutlich höhere Rißspitzenbeanspruchungsraten ergeben als bei dem initialen Belastungsvorgang. Die Rißspitzenbeanspruchungsraten bei der Belastung hängen von der Länge der Anfangskerbe und von der Ge-

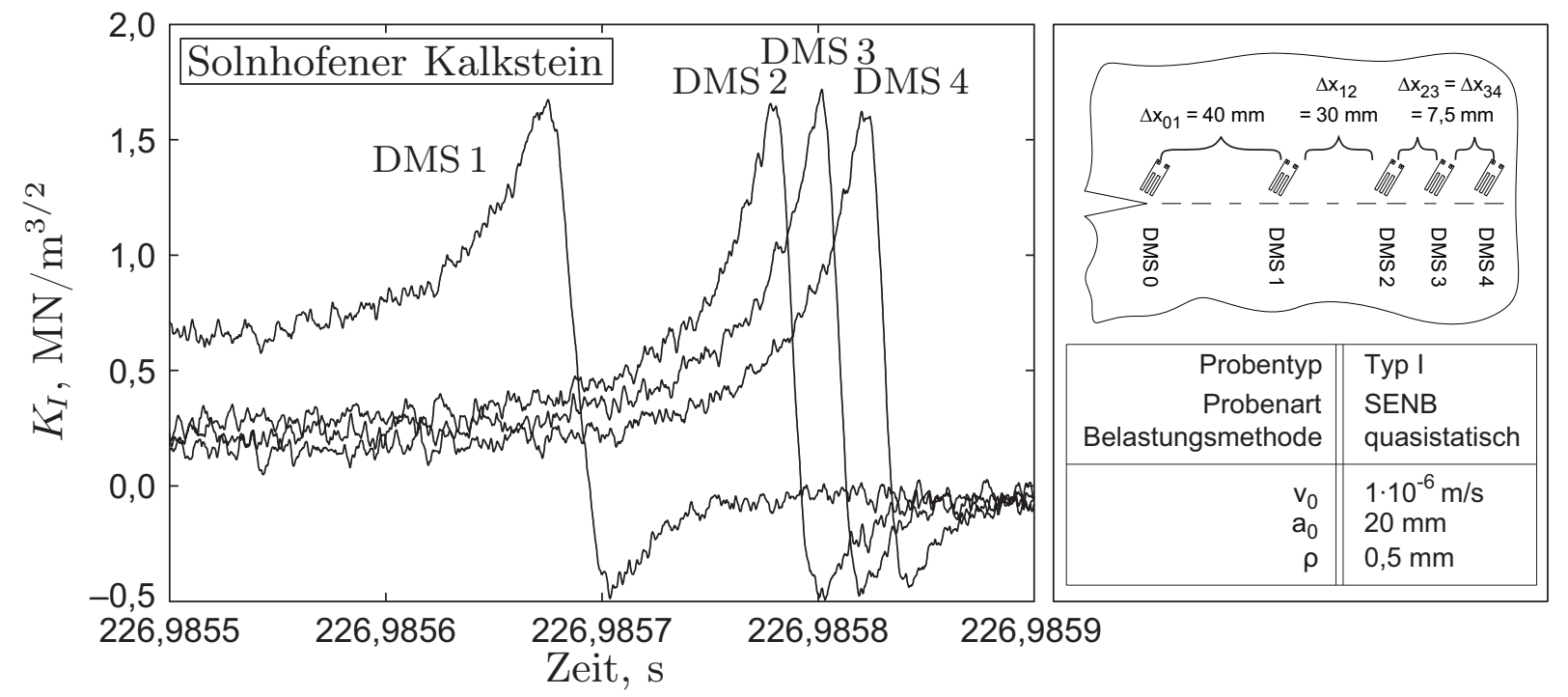

Abb. 41: Dehnungsmeßstreifen-Signale für schnelle überkritische Rißausbreitung in Solnhofener Kalkstein (Probe KE08), unter quasistatischer Beanspruchung initiiert. 
schwindigkeit des Belastungskolbens ab. Bei den verwendeten Geschwindigkeiten für den Belastungskolben von $v_{0}=10^{-6}-10^{-5} \mathrm{~m} / \mathrm{s}$ liegen die sich für Arkansas Novaculit einstellenden Beanspruchungsraten an der Rißspitze in der Größenordnung von $\dot{K}_{I}=10^{-2}-10^{-1} \mathrm{MN} / \mathrm{m}^{3 / 2} / \mathrm{s}$. Diese Beanspruchungsraten sind etwa doppelt so hoch wie für Solnhofener Kalkstein. Beanspruchungsraten von $\dot{K}_{I}=0,55-2,75 \mathrm{MN} / \mathrm{m}^{3 / 2} / \mathrm{s}$, wie sie in der ASTM-Prüfvorschrift E 399 [35] für Metalle vorgeschlagen werden, sind mit quasistatischer Belastung für die getesteten Gesteine nicht zu erreichen bzw. würde dadurch die Dauer eines Experimentes in den Bereich unter einer Sekunde sinken. Das gleiche gilt für Messungen, die analog der für Gesteine etablierten Methode zur Bruchzähigkeitsbestimmung [109] durchgeführt wurden (siehe Abs. 4.7, Seite 83). Eine Bewertung dieser und anderer Abweichungen von den ASTM-Normprüfvorschriften findet sich in [163]. Bei dynamischer Initiierung ergeben sich jedoch weitaus höhere Beanspruchungsraten.

Der in Abb. 42 unter dynamischen $\mathrm{Zug}$ (Modus-I)-Belastungsbedingungen mit einer Drei-Punkt-Biege-Anordnung (SENB-Probe) im Schlagbiegeexperiment mit dem Fallwerk an Solnhofener Kalkstein initiierte Rißlaufvorgang zeigt signifikant höhere Beanspruchungsraten für den Rißspitzendehnungsmeßstreifen (DMS 0). Insgesamt wurden mit dieser Initiierungsmethode, in Abhängigkeit von Schlaggeschwindigkeit und Starterkerblänge, Beanspruchungsraten von bis zu $\dot{K}_{I}=5 \cdot 10^{4} \mathrm{MN} / \mathrm{m}^{3 / 2} / \mathrm{s}$ erreicht. Dennoch stellen sich während des Rißausbreitungsprozesses wiederum höhere Rißspitzenbeanspruchungsraten ein. Es ist zu erkennen, daß der Riß sehr schnell nach der Initiierung eine hohe Ausbreitungsgeschwindigkeit, mit gleichzeitig ansteigender
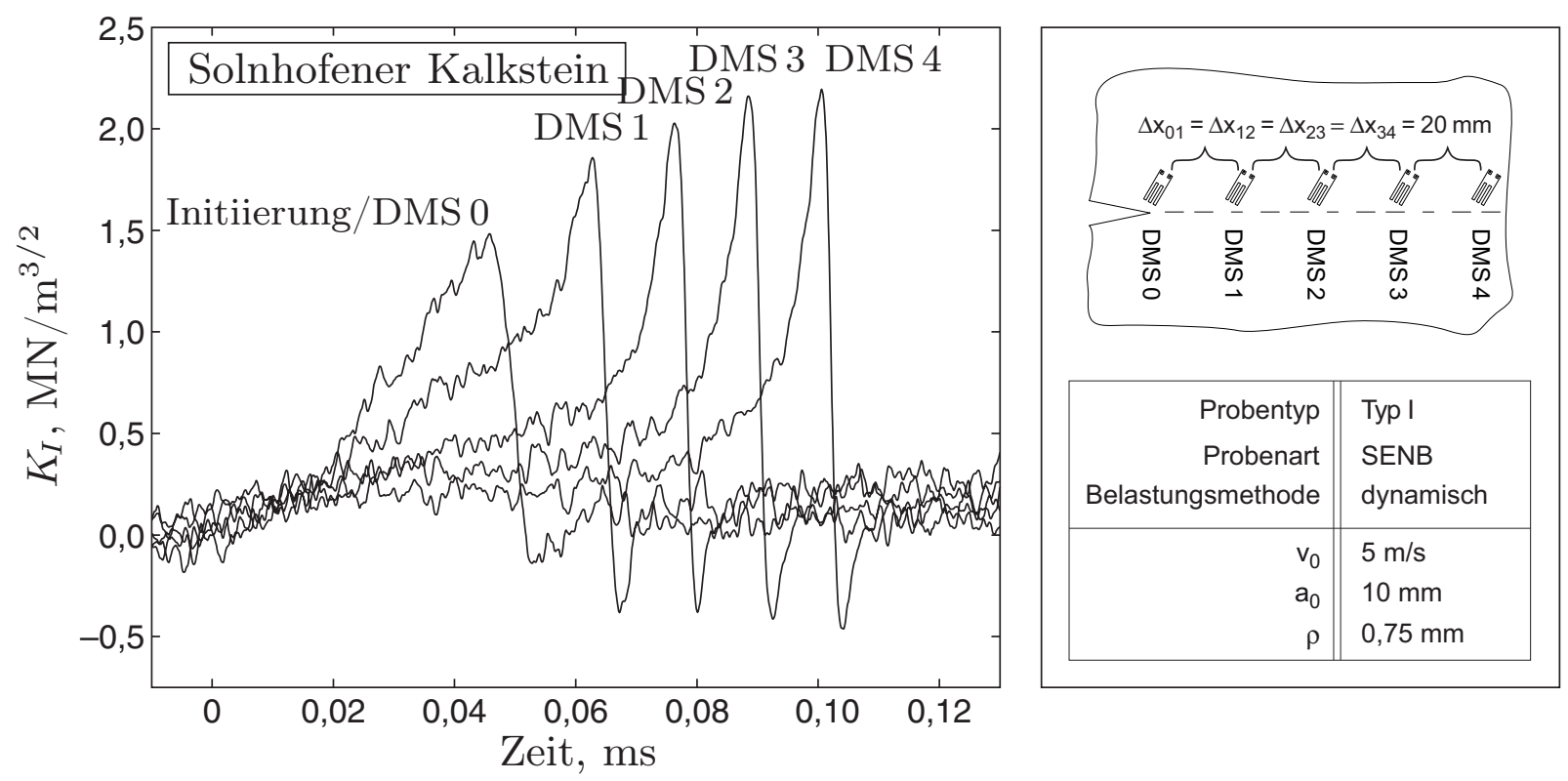

Abb. 42: Dehnungsmeßstreifen-Signale für schnelle Rißausbreitung in Solnhofener Kalkstein (Probe KD08), unter dynamischer Zug(Modus-I)-Belastung in der Fallgewichtsanlage initiiert. 
Rißlaufzähigkeit, annimmt. Dabei vollzieht sich die Initiierung des Risses auf einem höheren Niveau an Spannungsintensität als im quasistatischen Experiment (vergl. Abb. 39).

In Abb. 43 ist je ein Rißlaufexperiment, initiiert unter dynamischen Scher(ModusII)-Belastungsbedingungen mit der LECEI-Testmethode, für jedes der getesteten
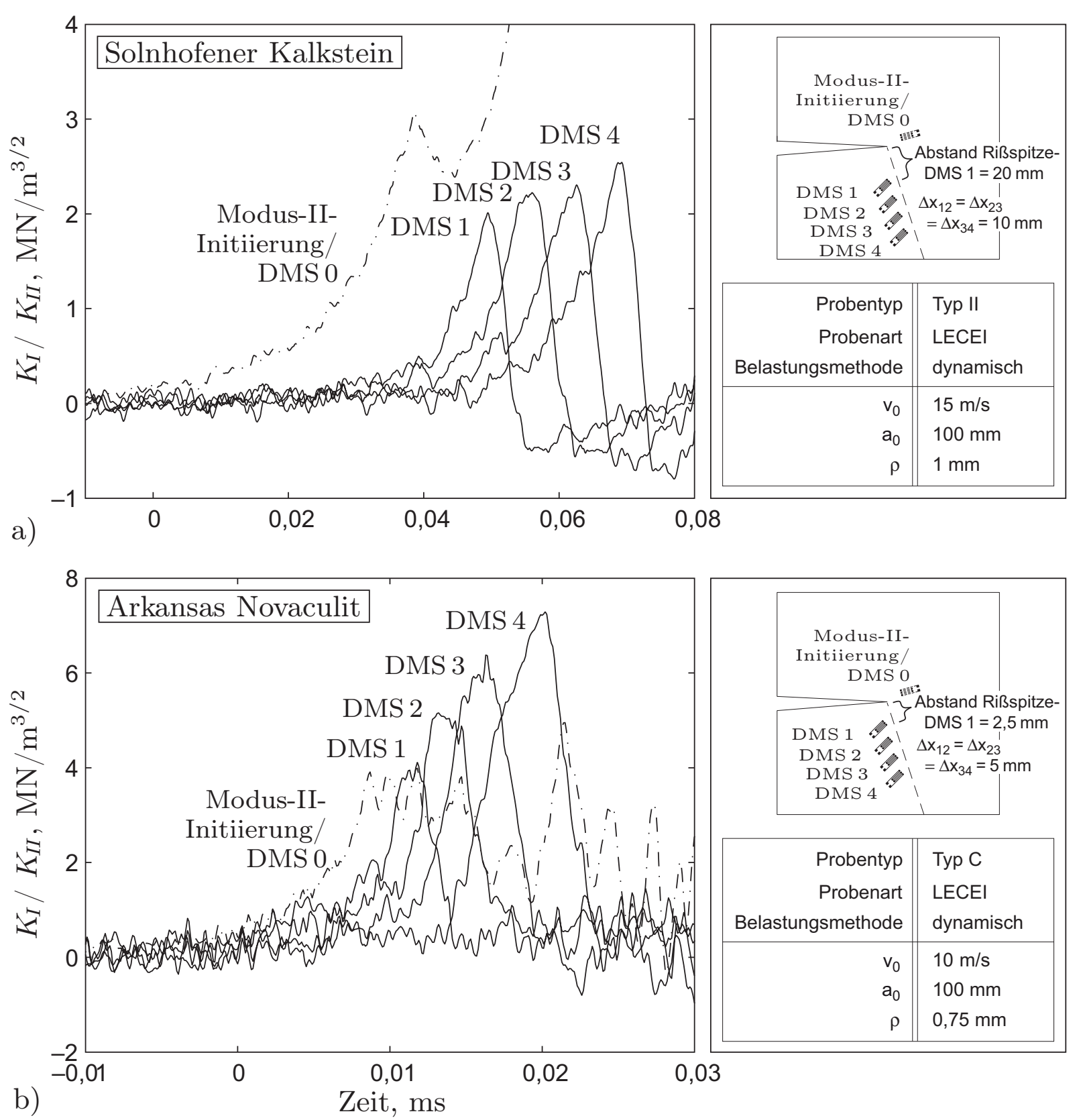

Abb. 43: Dehnungsmeßstreifen-Signale für LECEI-Testmethode: a) Solnhofener Kalkstein und b) Arkansas Novaculit. Die gestrichelte Signalkurve kennzeichnet die Scher(Modus-II)-Initiierung, die restlichen Signale den unter Zug(Modus-I)-Bedingungen laufenden Riß. 
Gesteinsmaterialien abgebildet. Wiederum erfolgt die Initiierung sehr rasch, nachdem die Rißspitzenbeanspruchung einsetzt. Bei der folgenden Rißausbreitung stellen sich sehr schnell hohe Ausbreitungsgeschwindigkeiten ein. Der Vergleich der Rißspitzendehnungsmeßstreifen (DMS 0, in der Abbildung strichpunktiert dargestellt) mit den Dehnungsmeßstreifen, die den Rißausbreitungsvorgang wiedergeben (DMS 1-4) zeigt, daß die Beanspruchungsraten, die während der Initiierung vorliegen, wie erwartet, ein sehr hohes Niveau erreichen und mit denjenigen vergleichbar sind, die sich während der Rißausbreitung einstellen. Auch bei dynamischer Scher(Modus-II)Initiierung vollzieht sich der Aufbau von Spannungsintensitäten bei Arkansas Novaculit doppelt so schnell wie für Solnhofener Kalkstein. Die entsprechenden Beanspruchungsraten liegen in der Größenordnung von $\dot{K}_{I I}=2 \cdot 10^{5} \mathrm{MN} / \mathrm{m}^{3 / 2} / \mathrm{s}$ (Arkansas Novaculit) bzw. $\dot{K}_{I I}=1 \cdot 10^{5} \mathrm{MN} / \mathrm{m}^{3 / 2} / \mathrm{s}$ (Solnhofener Kalkstein). Zudem wird in Arkansas Novaculit schneller ein hohes Geschwindigkeitsniveau mit den entsprechenden hohen Rißlaufzähigkeiten erreicht als in Solnhofener Kalkstein (vergl. Abs. 4.3, Abb. 38).

\subsection{Gesamtergebnisse}

Die beschriebenen Messungen decken den gesamten Geschwindigkeitsbereich, von der Initiierung bis zum Erreichen der maximalen Rißlaufgeschwindigkeit bei Einsetzen von Bruchgabelung ab. Sämtliche Ergebnisse der Meßreihen sind in Abb. 44 für Solnhofener Kalkstein und Abb. 45 für Arkansas Novaculit angegeben (die Daten der Experimente sind in Anhang $\mathrm{F}$ tabelliert).

Insgesamt werden in den untersuchten Gesteinen Rißausbreitungsgeschwindigkeiten bis in den Bereich von $v_{c}=2000 \mathrm{~m} / \mathrm{s}$ bei Solnhofener Kalkstein und von $v_{c}=2400 \mathrm{~m} / \mathrm{s}$ bei Arkansas Novaculit erreicht. Diese mit den Dehnungsmeßstreifen-Ketten bestimmten Rißausbreitungsgeschwindigkeiten werden durch die Ergebnisse der Auswertung von Hochgeschwindigkeitsphotos bestätigt. Die Meßergebnisse weisen, wie erwartet (siehe Abb.18), einen starken Anstieg der Rißlaufzähigkeiten bei hohen Rißausbreitungsgeschwindigkeiten auf. Ein zweiter starker Anstieg wird bei sehr geringen Rißausbreitungsgeschwindigkeiten beobachtet. Zwischen diesen Bereichen hoher Gradienten in der Geschwindigkeits-Bruchzähigkeits-Beziehung bildet sich bei Geschwindigkeiten von etwa $1 \mathrm{~m} / \mathrm{s}$ bis $1500 \mathrm{~m} / \mathrm{s}$ ein Plateau aus, für das große Geschwindigkeitsänderungen, ungeachtet der Streuung der Meßwerte, eine relativ geringe Erhöhung der Rißlaufzähigkeit bewirken. Bei beiden Gesteinsmaterialien ergibt sich, wie durch die in den Abbildungen angegebenen Ausgleichskurven angezeigt, prinzipiell der gleiche Verlauf der Geschwindigkeits-Bruchzähigkeits-Beziehung. Der gesamte Anstieg der Rißlaufzähigkeiten, von der Initiierung bis zur maximalen Rißlaufgeschwindigkeit, beträgt bei beiden Gesteinen jeweils annähernd eine Größenordnung. Jedoch sind die Niveaus der Bruchzähigkeiten, die für die unterschiedlichen Gesteine bestimmt wurden, um den Faktor zwei verschieden. Die niedrigsten Bruchzähigkeiten 


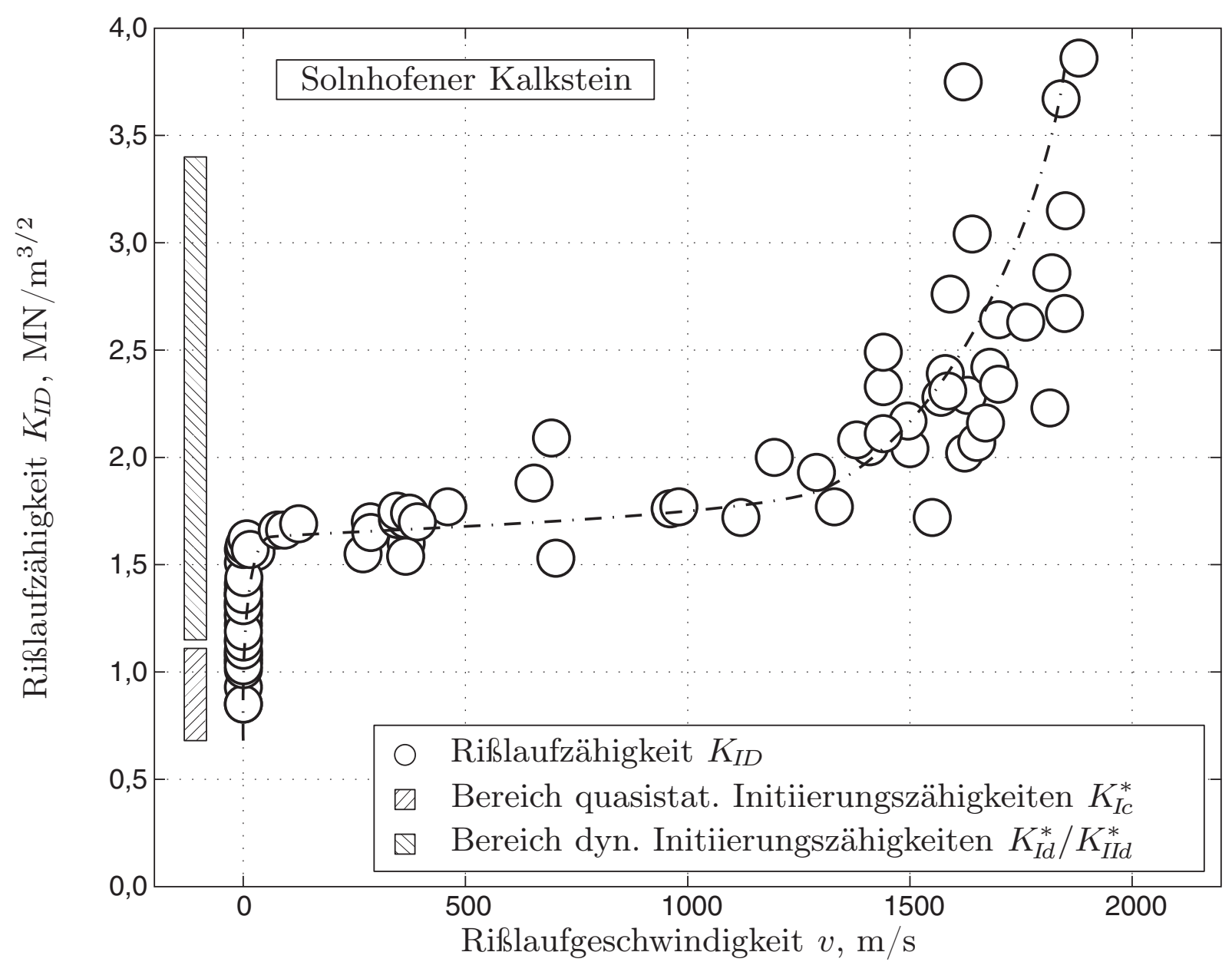

Abb. 44: Ergebnisdaten zu den Rißlaufereignissen in Solnhofener Kalkstein.

ergeben sich, wie im folgenden Absatz beschrieben, für die Initiierung, der die Rißlaufgeschwindigkeit $v_{c}=0$ zuzuordnen ist. Das Plateau, auf das die Rißlaufzähigkeiten bei der anfänglichen Rißbeschleunigung ansteigen, beträgt für Arkansas Novaculit $K_{I D} \approx 3 \mathrm{MN} / \mathrm{m}^{3 / 2}$ während für Solnhofener Kalkstein lediglich $K_{I D} \approx 1,5 \mathrm{MN} / \mathrm{m}^{3 / 2}$ bestimmt wurden. Auch die höchsten Rißlaufzähigkeiten, die im Bereich der maximalen Rißlaufgeschwindigkeit gemessen wurden, sind mit $K_{I D} \approx 8 \mathrm{MN} / \mathrm{m}^{3 / 2}$ für Arkansas Novaculit doppelt so hoch wie für Solnhofener Kalkstein mit $K_{I D} \approx 4 \mathrm{MN} / \mathrm{m}^{3 / 2}$.

Die in den Experimenten bestimmten Initiierungsbruchzähigkeiten sind zum Vergleich, versetzt neben den Rißlaufzähigkeiten mit niedriger Ausbreitungsgeschwindigkeit, in den Abbn. 44 und 45 angegeben, getrennt in Bereiche quasistatischer und dynamischer Initiierungszähigkeiten. Die Rißinitiierungszähigkeiten in den Experimenten der vorliegenden Arbeit wurden ausschließlich an Starterkerben mit einer endlichen Kerbschärfe bestimmt. Da gültige Bruchzähigkeiten nur an Rissen mit einer scharfen Spitze bestimmt werden, sind die Initiierungszähigkeiten von Kerben nur relativ zueinander interpretierbar, gegenüber Bruchzähigkeiten von scharfen Rissen müßten sie eher zu niedrigeren Werten korrigiert werden. Die gemessenen Initiie- 


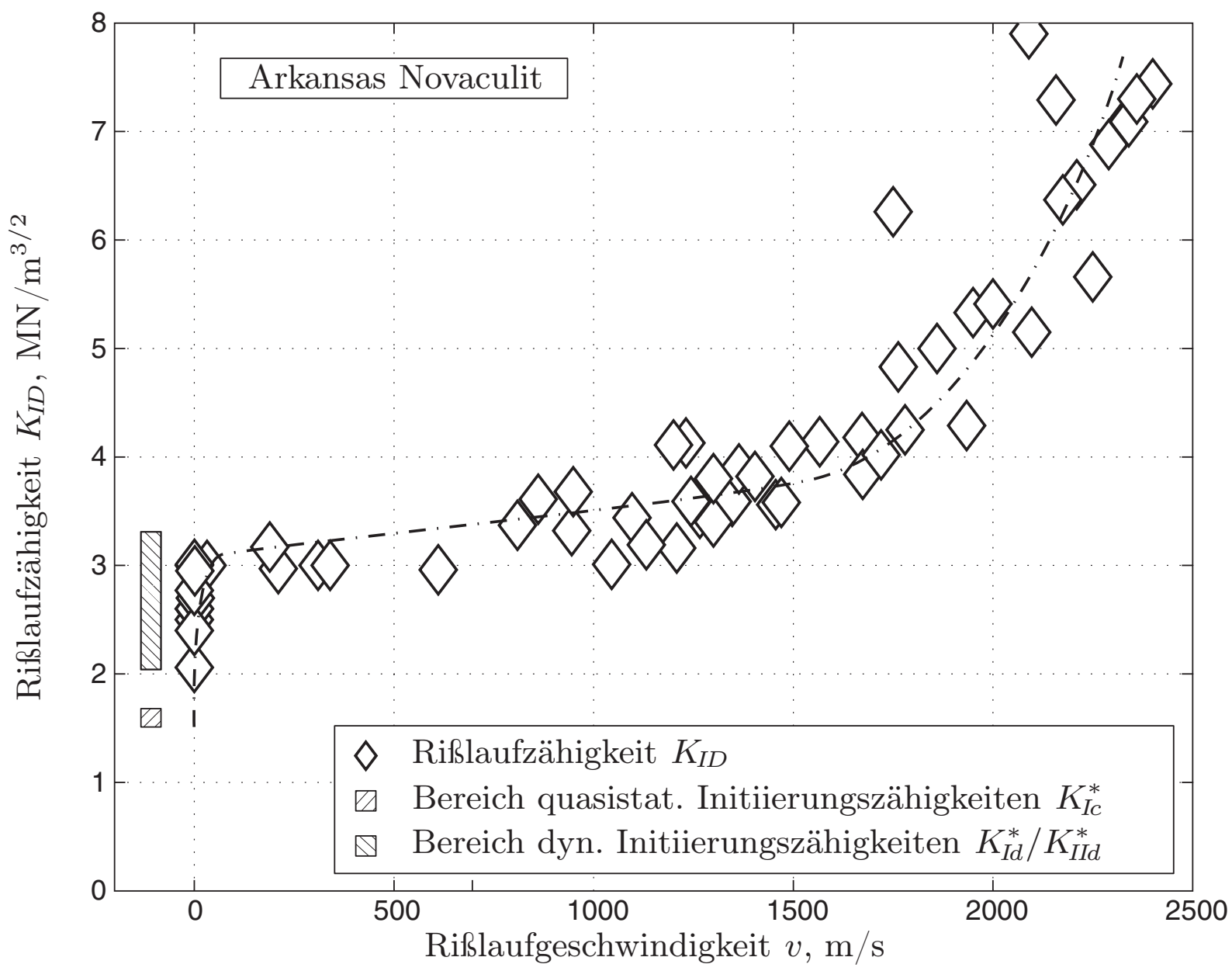

Abb. 45: Ergebnisdaten zu den Rißlaufereignissen in Arkansas Novaculit.

rungszähigkeiten sind daher entsprechend kenntlich gemacht und werden als $K_{I c}^{*}$ für quasistatische Belastung sowie als $K_{I d}^{*}$ und $K_{I d}^{*}$ für dynamische Schlagexperimente angegeben. Die erzielten Ergebnisse für quasistatische Initiierung an Solnhofener Kalkstein sind: $K_{I c}^{s *}=0,86 \pm 0,15 \mathrm{MN} / \mathrm{m}^{3 / 2}$ (als Schwankungsbreite ist die Standardabweichung vom Mittelwert angegeben, Kennzeichnung "s، für den Beginn stabiler subkritischer Rißausbreitung). Diese Werte decken sich sehr gut mit den Literaturdaten dieser Größe (Tabelle 2), wobei sogar, trotz der Verwendung abgerundeter Kerben, teilweise geringere als die tabellierten Bruchzähigkeiten auftraten. Die Meßdaten für Arkansas Novaculit liegen mit $K_{I c}^{s *}=1,60 \pm 0,10 \mathrm{MN} / \mathrm{m}^{3 / 2}$ ebenfalls innerhalb bzw. an der oberen Grenze der publizierten Ergebnisse. Eine Abhängigkeit von der Anfangsrißlänge bzw. von der Belastungsgeschwindigkeit konnte nicht festgestellt werden. Für die dynamischen Initiierungsmethoden wurden Bruchzähigkeiten von $K_{I d}^{*}=1,55 \pm 0,70 \mathrm{MN} / \mathrm{m}^{3 / 2}$ und $K_{I I d}^{*}=3,20 \pm 0,20 \mathrm{MN} / \mathrm{m}^{3 / 2}$ (Solnhofener Kalkstein) sowie von $K_{I d}^{*}=2,40 \pm 0,40 \mathrm{MN} / \mathrm{m}^{3 / 2}$ und $K_{I I d}^{*}=2,50 \pm 0,40 \mathrm{MN} / \mathrm{m}^{3 / 2}$ (Arkansas Novaculit) gemessen. Ein Einfluß der Anfangsrißlänge oder der Belastungs- bzw. Schlaggeschwindigkeit $v_{0}$ und der damit einhergehenden Steigerung der Beanspruchungsrate 
$\dot{K}$ ist für die dynamischen Initiierungszähigkeiten ebenfalls nicht zu beobachten. Der Vergleich der Initiierungsmethoden untereinander zeigt jedoch, daß für dynamische Initiierung regelmäßig höhere Initiierungsbruchzähigkeiten als mit quasistatischer Initiierung bestimmt wurden. Für diesen Effekt muß die Belastungsgeschwindigkeit $v_{0}$ und somit die Beanspruchungsrate um mehrere Größenordnungen bis in den Bereich von $\dot{K}>10^{4} \mathrm{MN} / \mathrm{m}^{3 / 2} / \mathrm{s}$ (s. Abs. 4.4.2) gesteigert werden. Diese Beobachtung deckt sich mit früheren Betrachtungen für spröde Materialien [175]. Die höchsten Initiierungszähigkeiten wurden mit der LECEI-Initiierungsmethode unter dynamischen Scher(Modus-II)-Belastungsbedingungen erzielt. Zum Vergleich an Solnhofener Kalkstein unter quasistatischen Belastungsbedingungen bestimmte Scher(Modus-II)Initiierungszähigkeiten betragen $K_{I I c}^{*}=1,00 \pm 0,10 \mathrm{MN} / \mathrm{m}^{3 / 2}$ (diese liegen geringfügig über den für Modus-I bestimmten Werten).

Im Anschluß an die Initiierung steigen, wie bereits erwähnt, unter den vorgegebenen Bedingungen konstanter Durchbiegungsraten die Rißlaufzähigkeiten bis zum Erreichen eines Niveaus an, für das trotz fortgesetzter Rißbeschleunigung konstante Rißlaufzähigkeiten bestimmt werden. Der Geschwindigkeitsbereich, für den der Übergang auf das konstante Niveau erfolgt, hängt von den jeweiligen Materialeigenschaften ab und ist bei den untersuchten Gesteinen unterschiedlich. Wie in Abb. 46 gezeigt, vollzieht sich dieser Übergang bei Solnhofener Kalkstein bei Rißausbreitungsgeschwindigkeiten von etwa $v_{c} \approx 1 \mathrm{~m} / \mathrm{s}$, während sich konstante Rißlaufzähigkeiten für Arkansas Novaculit schon bei $v_{c} \approx 0,1 \mathrm{~m} / \mathrm{s}$ einstellen.

Trotz der Unterschiede der Beträge der Ergebnisdaten besteht eine prinzipielle Übereinstimmung des Verlaufs der Ergebniskurven der beiden untersuchten Gesteine. Dies zeigt sich bei der Normierung der Meßkurven aus Abb. 44 und Abb. 45 auf die jeweilige Rißinitiierungsbruchzähigkeit $K_{I c}^{i}$ (siehe Kapitel 5) und die jeweilige maximale Rißlaufgeschwindigkeit $v_{c}^{\max }$ besonders deutlich (Abb. 47).

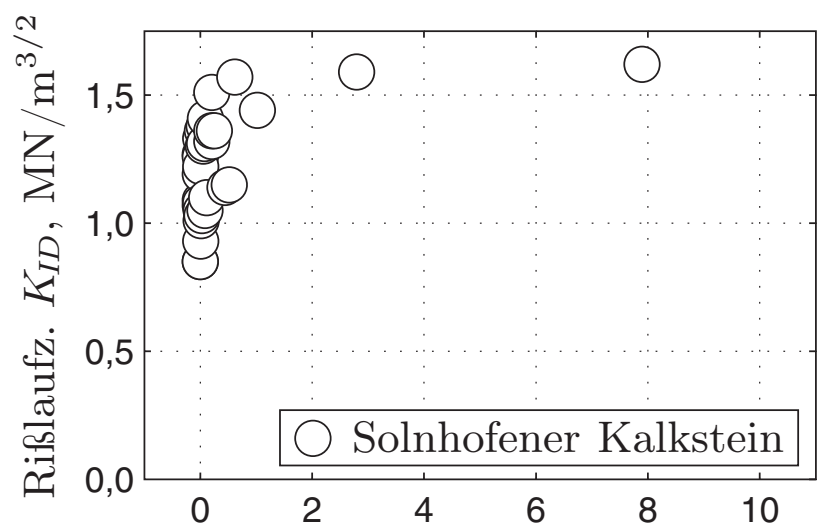

a)

Abb. 46: Ausschnitt aus den Ergebnisdaten für geringe Rißlaufgeschwindigkeiten für: a) Solnhofener Kalkstein und b) Arkansas Novaculit. 


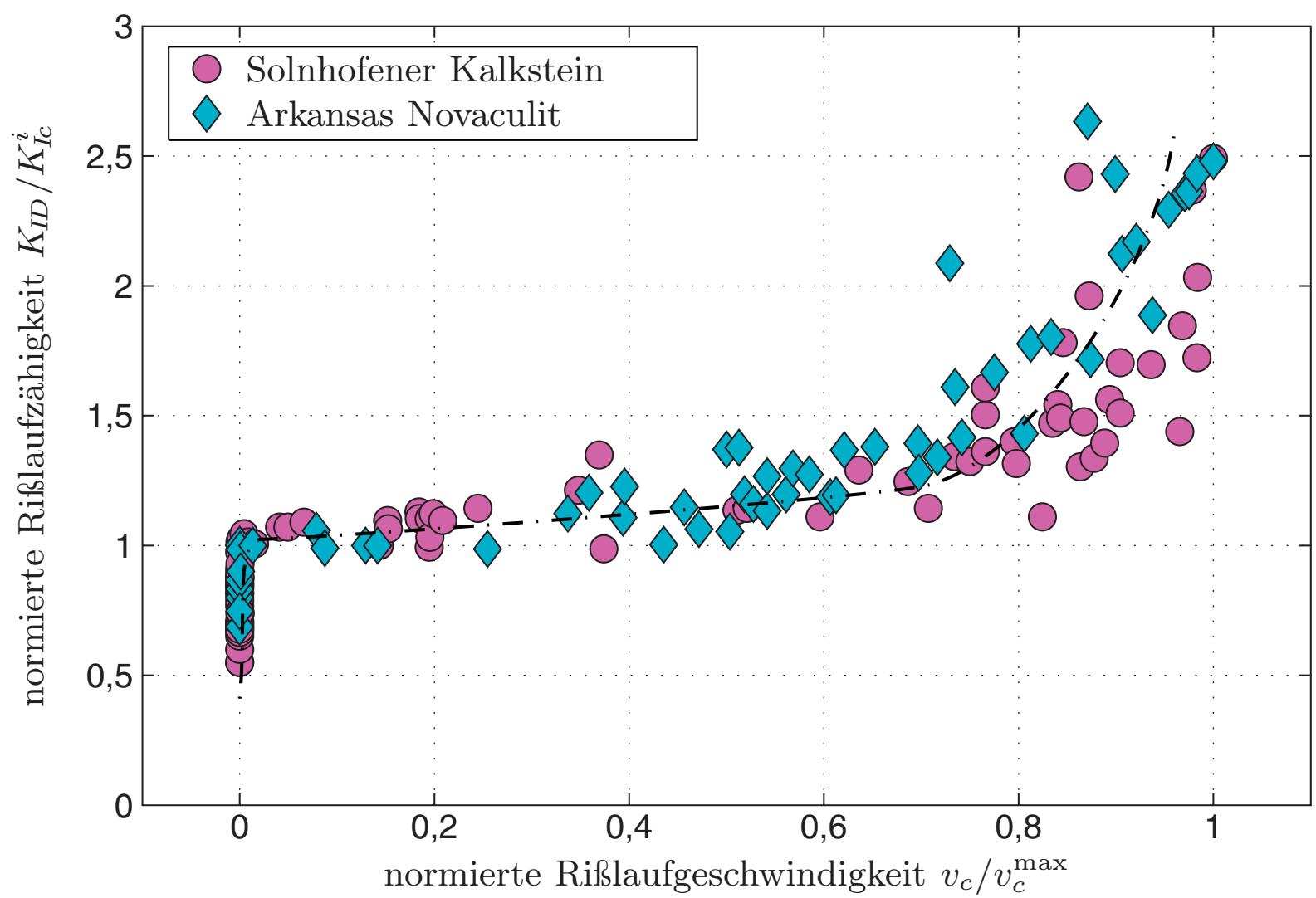

Abb. 47: Ergebnisdaten Arkansas Novaculit und Solnhofener Kalkstein normiert auf die jeweilige Initiierungszähigkeit $K_{I c}^{i}$ und die maximale Rißlaufgeschwindigkeit $v_{c}^{\max }$.

\subsection{Vergleich der Ergebnisse durch mikroskopische Untersu- chungen}

Die Unterschiede des Bruchverhaltens der beiden Gesteine betreffen - wie vorstehend beschrieben - zwei Größen: Auf der einen Seite die Höhe der Bruchzähigkeit, die in den Experimenten bestimmt wurde und auf der anderen Seite die Geschwindigkeitswerte, für die sich der Übergang von der subkritischen Rißerweiterung zum überkritischen Bereich vollzieht $\left(v_{c}^{t}\right)$ sowie diejenigen der maximalen Rißausbreitungsgeschwindigkeit. Diese Unterschiede sind nicht allein auf die unterschiedlichen bruchmechanischen Eigenschaften der entsprechenden Mineralien als Ausgangsmaterial der untersuchten Gesteine zurückzuführen. Zwar ist die Bruchzähigkeit von Quarz deutlich höher als von Calcit [163], jedoch müssen für eine Bewertung der Unterschiede im Bruchverhalten wegen der beobachteten Bedeutung der Energiedissipation in der Rißspitzenprozeßzone auch mikromechanische Effekte berücksichtigt werden [30]. Dazu sind in Abb. 48 vergleichend Bilder von Rißlaufereignissen in beiden Gesteinen dargestellt, die mit der Methode der SekundärelektronenEmission (SEM) mit einem Rasterelektronenmikroskop aufgenommen wurden. Die 

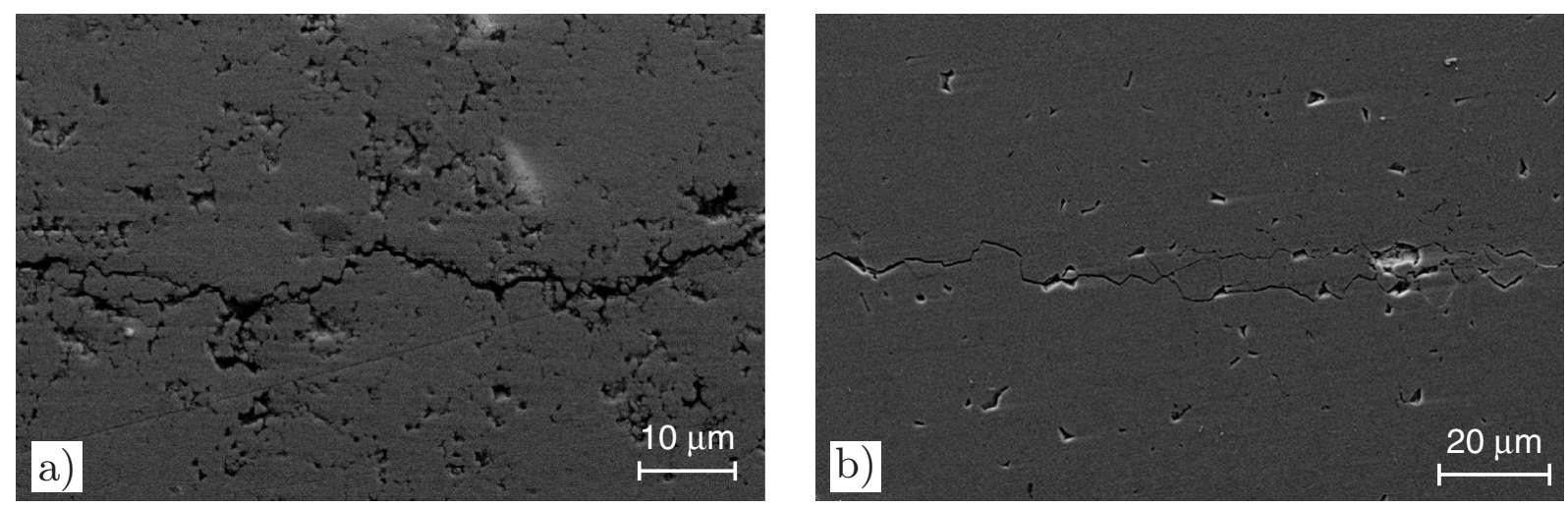

Abb. 48: Rasterelektronenmikroskop-Aufnahmen (SEM) von Rißlaufereignissen in: a) Solnhofener Kalkstein und b) Arkansas Novaculit (Rißlaufrichtung von links nach rechts).

Mikroskopbilder zeigen, daß für beide Gesteine die Rißausbreitung hauptsächlich entlang der Korngrenzen erfolgt, wobei der Riß teilweise auf zwei Seiten um einzelne oder mehrere Körner herumläuft. Unterschiede zwischen Solnhofener Kalkstein (Abb.48a) und Arkansas Novaculit (Abb. 48b) zeigen sich allerdings in der Korngröße und der Porosität. Die Korngrößen, und damit die Länge der Schwächezonen an den Korngrenzen, an denen sich Mikrorisse bilden können, von Arkansas Novaculit sind größer als in Solnhofener Kalkstein (siehe Tabelle 2). Dadurch bestehen in Arkansas Novaculit günstigere Bedingungen, sowohl für Bildung einer ausgedehnten Prozeßzone als auch für die Ausbreitung des Makrorisses durch fortlaufende Verbindung von Mikrorissen. Weiterhin weist Solnhofener Kalkstein mit $\phi=4,0 \%$ eine höhere Porosität auf als Arkansas Novaculit mit $\phi=0,1 \%$. Die Porositäten wurden stichpunktartig in Imbibitionsexperimenten vor der Versuchsdurchführung bestimmt. Ursächlich für die größere Höhe der Porosität ist, daß in Solnhofener Kalkstein präexistierende Poren in Form ausgedehnter Hohlräume vorliegen (Abb.48a). Demgegenüber ist die Porosität bei Arkansas Novaculit an die Korngrenzen gebunden, die Poren haben dadurch die Form flacher Schlitze geringen Volumens. Die im Mikroskopbild von Arkansas Novaculit (Abb. 48b) sichtbaren Hohlräume sind Artefakte, entstanden dadurch, daß bei der Präparation des Dünnschliffes Kristalle aus der Probenoberfläche herausgebrochen sind. Im Gegensatz zu Arkansas Novaculit wird daher bei Solnhofener Kalkstein die Ausbreitung und Beschleunigung von Rissen durch die Porosität behindert. Dies ist in Übereinstimmung mit dem beobachteten Verhalten und erklärt zudem das unterschiedliche Initiierungsverhalten, bei dem für Arkansas Novaculit im Gegensatz zu Solnhofener Kalkstein zu Beginn der Rißausbreitung die Tendenz zu sprunghaftem Rißfortschritt (pop-in, s. o.) besteht.

In Abb.48, ebenso wie in einer Vielzahl weiterer Mikroskopaufnahmen, ist eine Schädigungszone entlang des Rißpfades nicht zu erkennen (vergl. Abb.9, siehe auch [34]). Da aufgrund der geringen Korngrößen der in den Hauptexperimenten getesteten Ge- 
steine die mikroskopische Detektion der Schädigungszone erschwert wird, wurden hierzu Untersuchungen an grobkörnigerem Marmor durchgeführt. Der untersuchte Marmor ist ebenfalls sehr homogen und besteht wie Solnhofener Kalkstein zu $99 \%$ aus Calcit. Calcit besitzt eine für geologische Minerale vergleichsweise hohe Fähigkeit große Spannungen durch plastische Verformung abzubauen. Dies geschieht u. a. über Zwillingsbildung. Lichtmikroskopische Aufnahmen einer angeschnittenen Bruchfläche zeigen daher neben der Mikrorißbildung dieses Phänomen (s. Abb.49a). Transgranulare Brüche sind nur in Ausnahmefällen zu beobachten. Die Probe wurde vor der Präparation des Dünnschliffes mit einem farbigen Epoxidharz imprägniert, um eine weitere Materialschädigung auszuschließen und das Auffinden von Mikrorissen zu erleichtern [176]. Für die Aufnahme wurde polarisiertes Licht verwendet und der Kontrast durch die Änderung der dadurch ursprünglich erzeugten Farbgebung erhöht. Die laterale Erstreckung der Schädigungszone, die bei der Analyse der Deformationen entlang der Bruchfläche bestimmt wurde, lag unter dem erwarteten Wert von $r_{p}=0,4 \mathrm{~mm}$, der sich aus der bruchmechanischen Abschätzung mit Gl. (7) $\left(\sigma_{t}=12 \mathrm{MN} / \mathrm{m}^{2}\right.$ und $\left.K_{I c}=0,6 \mathrm{MN} / \mathrm{m}^{3 / 2}\right)$ und der für das bloße Auge sichtbaren Ausdehnung ergibt (der abgebildete Dünnschliff stammt aus der Probenoberfläche des in Abb. 60 in Anhang A gezeigten Experimentes). Erschwert wird die Bestimmung der Größe der Schädigungs- bzw. der Prozeßzone entlang des Rißufers des makroskopischen Zug(Modus-I)-Risses dadurch, daß Korngrenzen-Mikrorisse in dem vorliegenden Material generell nur schwer detektierbar sind, zumal eine erkennbare Rißöffnung nach Abklingen der Beanspruchung bei den im Labor herrschenden Umgebungsbedingungen nicht persistent ist. Somit entsteht kein deutlicher Kontrast zu den im Material enthaltenen, nicht durch den Bruchvorgang verursachten sonstigen Mikrorissen (siehe [29]; vergl. auch Bilder des Belastungsverlaufs in Granitexperiment, Abb. 58 in Anh.A). Gleichzeitig wurde festgestellt, daß hohe Spannungen schon in geringer Entfernung zur Bruchfläche nicht mehr auftreten. Dies zeigt ein, in Abb.49b vergleichend zu dem Schnitt durch die Bruchfläche abgebildeter, Dünnschliff von Materialpartikeln, die während des Rißlaufexperimentes von der Bruchfläche abgelöst wurden. Dieses Bruchflächenmaterial wurde wesentlich stärker deformiert. Es
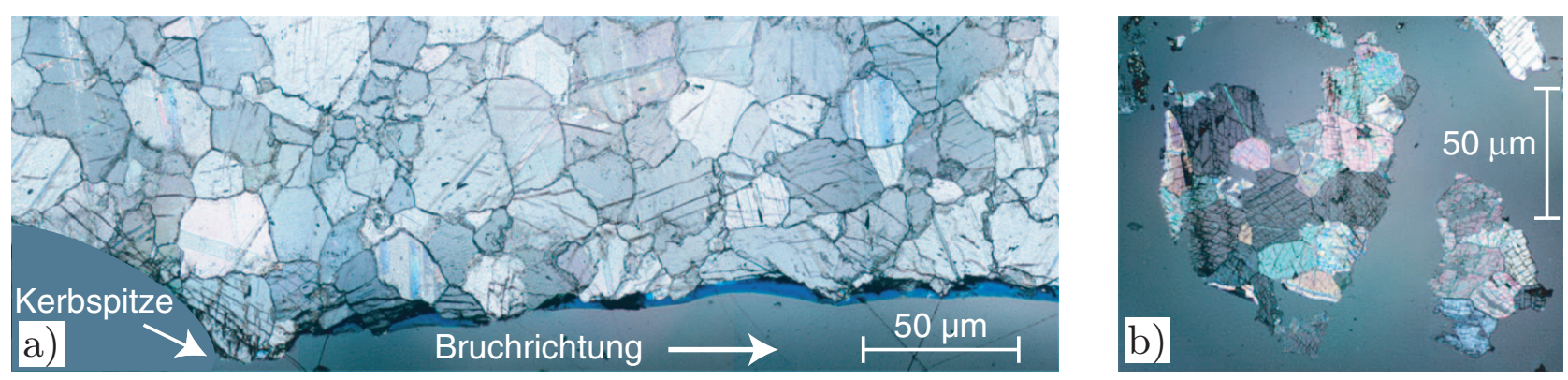

Abb. 49: Ausschnitt aus, in Durchlicht aufgenommenen, Mikroskopbildern eines Rißlaufereignisses in Marmor: a) senkrecht zur Bruchfläche und b) von der Bruchfläche abgelöstes Material. 
ist offensichtlich, daß die Spannungen, die zu diesen Deformationen und zur Ablösung von der Bruchfläche geführt haben, signifikant höher als die für Abb.49a beobachteten gewesen sind. Diese Beobachtungen werden sowohl durch die Analyse von akustischen Emissionen bei der Mikrorißbildung im Verlauf der Rißausbreitung in Laborproben (siehe z. B. [34,107, 177]) als auch durch mikroskopische Untersuchungen an exhumierten natürlichen Rißlaufereignissen bestätigt, für die unter günstigen in-situ-Bedingungen die Mikrorisse der Prozeßzone durch Mineralalteration detektierbar bleiben [178]. 


\subsection{Kontroll- und Vergleichsmessungen}

Bruchenergiemessungen mit dem Globalenergie-Verfahren in Schlagbiegeversuchen

Kontrollmessung zur Erhöhung der Rißlaufzähigkeiten bei hohen Rißlaufgeschwindigkeiten wurden an Proben sehr geringer Abmessungen vom Charpy-Typ III (s. Tabelle 3) an Solnhofener Kalkstein durchgeführt. Die Proben waren mit Initiierungskerben unterschiedlicher Länge von $a_{0}=1 \mathrm{~mm}$ bis $a_{0}=5 \mathrm{~mm}$ versehen und wurden in einem instrumentierten Pendelschlagwerk (Typ Wolpert, Fallmasse $20 \mathrm{~kg}$ ) bei Schlaggeschwindigkeiten von $0,25 \mathrm{~m} / \mathrm{s}$ getestet. Als Beispiel ist ein repräsentatives Lastsignal ist in Abb. 50a über der Zeit $t$ und über der Durchbiegung $s$ dargestellt. Deutlich sind hier die in Abs. 3.3.2 angesprochenen Schwingungen ausgeprägt. Bei

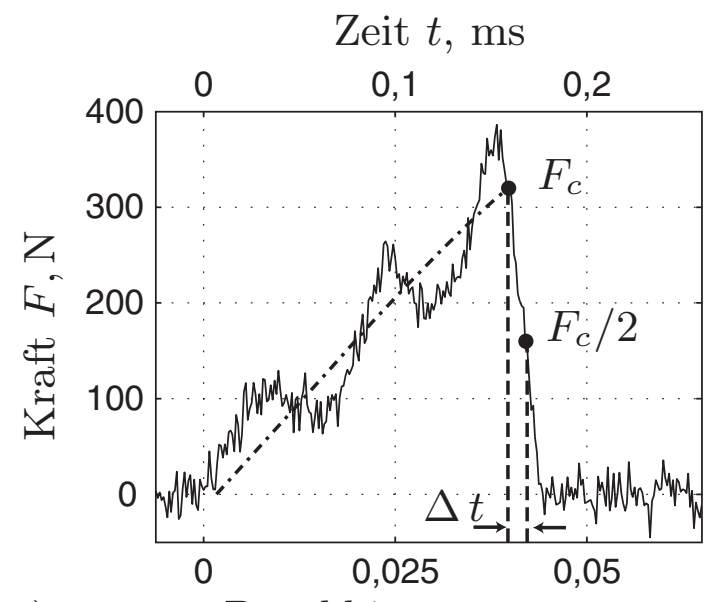

a)

Durchbiegung $s, \mathrm{~mm}$

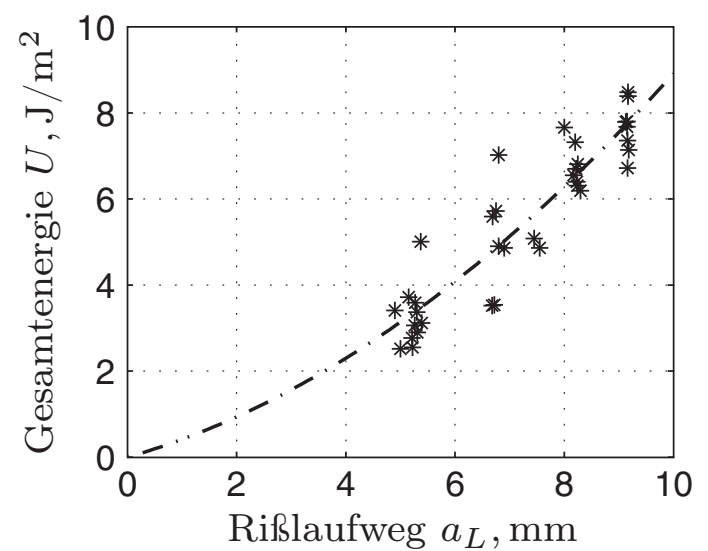

b)

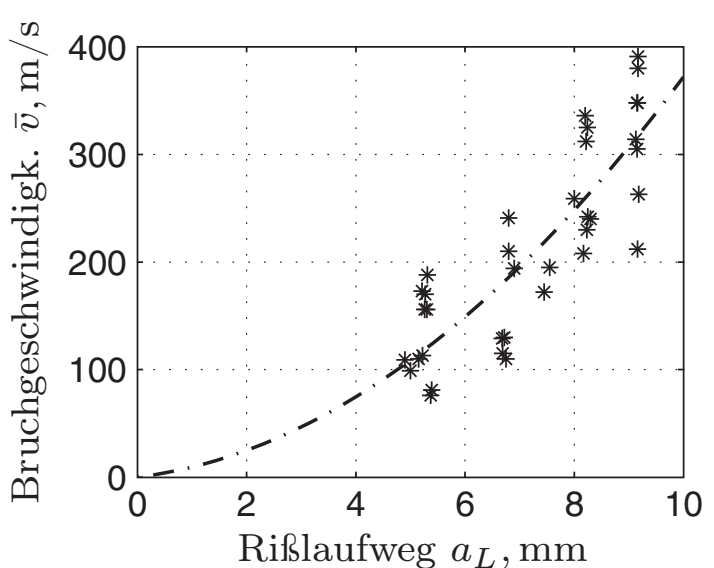

c)

Abb. 50: a) Versuchsergebnis zur Ermittlung von Globaldaten durch instrumentierten Schlagbiegeversuch sowie Ergebnisse des Globalenergie Verfahrens bei unterschiedlichen Rißlaufwegen für b) die Proben-Gesamtenergie und c) die gemittelte Rißgeschwindigkeit. 
der Bestimmung der kritischen Last, beim Versagen der Probe wurde daher eine Ausgleichskurve verwendet. Für die Integration der Schlagenergie ist diese Ausgleichskurve nicht relevant. Die Auswertung erfolgte entsprechend Abs. 3.4. Abbildung 50b zeigt die während der Belastung und dem Versagen von der Probe aufgenommene Gesamtenergie $U$ über dem Rißlaufweg $a_{L}$ (Ligamentlänge) und Abb. 50c die aus dem Kraftabfall für jedes Rißlaufereignis gewonnene mittlere Rißgeschwindigkeit $\bar{v}=\left(W-a_{0}\right) / 2 \Delta t=\left(a_{L}\right) / 2 \Delta t$. Aus der Änderung der Energie (Ausgleichskurve in Abb.50b) für unterschiedliche Rißlaufwegverlängerungen wurden Näherungen für kritische $G$ - bzw. $K$-Werte bestimmt.

Diese mit dem Globalenergie-Verfahren in Schlagbiegeversuchen an Charpy-Proben mittelnd erfassten Bruchenergiewerte $G_{I D}$ sind in Abb. 51 wiedergegeben. Die kritischen Werte der dynamischen Energiefreisetzungsrate wurden gemäß Gl. (15)a in dynamische Bruchzähigkeiten $K_{I D}$ umgewandelt. Eine Korrektur bei der Umrechnung muß wegen der geringen Bruchausbreitungsgeschwindigkeiten im Vergleich mit der Rayleighwellengeschwindigkeit nicht vorgenommen werden. Zusätzlich sind die nach dem gleichen Verfahren gewonnenen Werte für Rißinitiierung bestimmt sowie die entsprechend Abs. 3.4 nach dem ASTM-Normprüfverfahren (ASTM E 23) aus kritischen Kraftwerten $F_{c}$ bestimmten Initiierungszähigkeiten abgebildet. Obwohl in Abb. 51 nur Näherungswerte aus gemittelten Daten für Proben sehr kleiner Abmessungen dargestellt sind und deshalb kein direkter Vergleich der Absolutwerte mit den in den Abbn. 44 und 45 wiedergegebenen Daten erfolgen soll, wird tendenziell das glei-

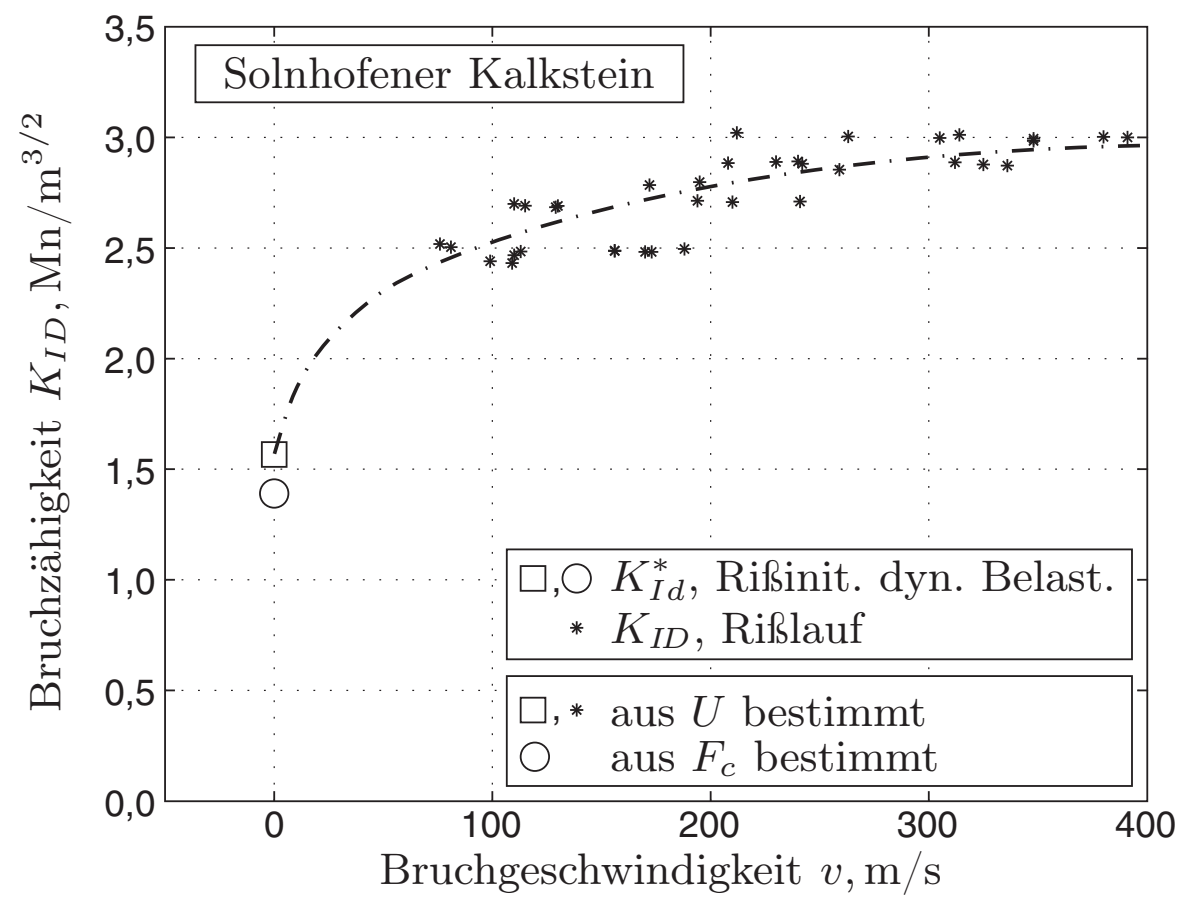

Abb. 51: Bruchzähigkeit für laufende Risse und Rißinitiierung (gemittelte Näherungswerte aus Schlagbiegeversuchen). 
che prinzipielle Verhalten beobachtet: Die Bruchzähigkeiten für laufende Risse sind deutlich größer als für Rißinitiierung. Auch die Initiierungszähigkeiten stimmen trotz der geringen Probengrößen mit den entsprechenden Werten der Hauptexperimente überein.

\section{Rißinitiierung und subkritische Rißausbreitung}

Eine weitere Serie von Kontrollexperimenten wurde unter Bedingungen langsamer subkritischer Rißerweiterung durchgeführt. Die Experimente wurden, angelehnt an die ISRM-Vorschriften, durch Vorgabe einer konstanten Rißöffnungsrate $\left(1 \cdot 10^{-7} \mathrm{~m} / \mathrm{s}\right.$ und $5 \cdot 10^{-7} \mathrm{~m} / \mathrm{s}$ ) durchgeführt. Die Proben waren von rechteckigem Querschnitt, die Starterkerben wurden wie in Abs. 4.1 beschrieben mit einem Sägeschnitt angebracht. Ein typisches Meßergebnis zeigt Abb. 52. Es wurden wiederholt EntlastungsWiederbelastungs-Schleifen durchgeführt. Last und Rißaufweitung COD (Abb. 52a) wurden im Gegensatz zur Durchbiegung $s$ (Abb. 52b) direkt an der Probe gemessen. Letztere wurde aus der Translation des Belastungskolbens bestimmt, wodurch sich hier Setzeffekte der Belastungsapparatur in einer anfänglichen Erhöhung der Steigung der Belastungskurve abbilden.

Das Beispiel zeigt inelastisches Verhalten, das bereits vor Erreichen der maximalen Last einsetzt. Die Auswertung der Meßserie gemäß den ISRM-Korrekturvorschriften liefert mit $K_{I c}=1,00 \pm 0,10 \mathrm{MN} / \mathrm{m}^{3 / 2}$ für Solnhofener Kalkstein eine korrigierte Rißinitiierungszähigkeit, die gegenüber den unkorrigierten Werten $25 \%$ erhöht ist, ohne allerdings die Streuung der Meßwerte zu verbessern. Dieser Bruchzähigkeitswert liegt im oberen Bereich der in den Hauptexperimenten erzielten Werte und

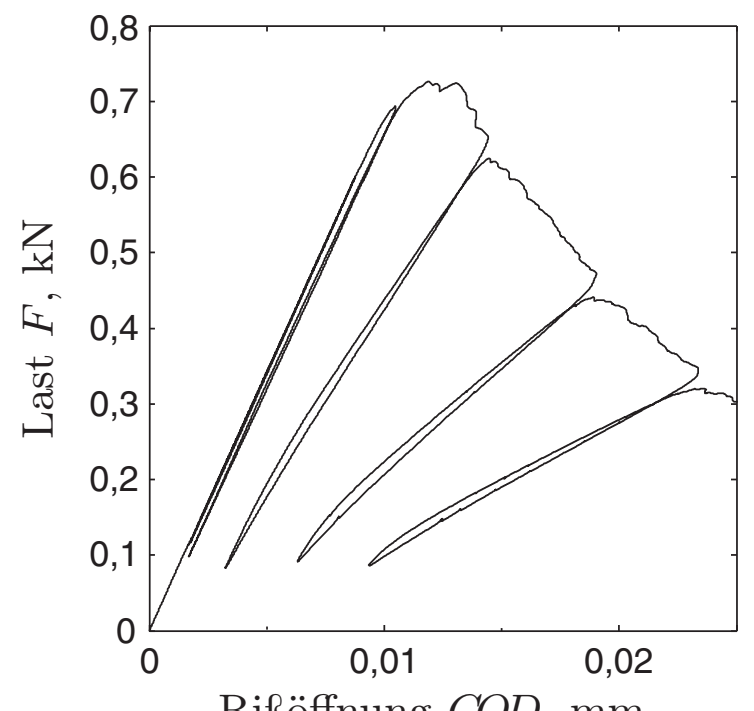

a)

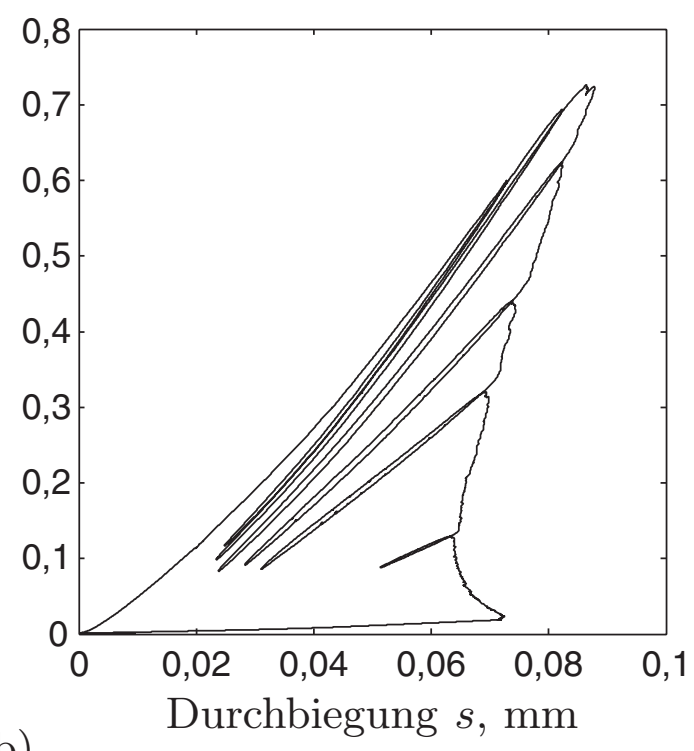

b)

Abb. 52: Typische Versuchsdaten für langsame subkritische Rißausbreitung in Solnhofener Kalkstein: a) als Funktion der Rißöffnung und b) als Funktion der Durchbiegung. 
der o. a. Referenzdaten. Für Arkansas Novaculit wurden Initiierungszähigkteiten von $K_{I c}^{*}=1,22 \pm 0,03 \mathrm{MN} / \mathrm{m}^{3 / 2}$ bestimmt, wobei das zuvor geschilderte ,pop-in“-Verhalten bei Regelung der Rißöffnungsrate nicht zu beobachten war. Diese Werte sind ebenfalls in Übereinstimmung mit den der Literatur entnommenen Meßdaten, decken sich allerdings innerhalb der Schwankungsbreiten nicht mit den in den Hauptexperimenten erzielten Ergebnissen. Die resultierenden Rißausbreitungsgeschwindigkeiten wurden im Mittel zu $v_{c}=1,10 \mathrm{~mm} / \mathrm{s}$ und $v_{c}=1,65 \mathrm{~mm} / \mathrm{s}$ bestimmt.

Gleichzeitig ist zu erkennen, daß im Nachbruchbereich zur Kontrolle des Ausbreitungsvorganges die Verformungsenergie reduziert werden muß. Die Ergebnisse zeigen somit, daß sich in sprödbrechendem Gestein bei konstanten Verformungsraten langsame subkritische Rißerweiterung ausbildet. Kontrollierte Rißerweiterung während eines Rißlaufereignisses in einem herkömmlichen sprödbrechenden Strukturmaterial zu erzeugen ist nicht möglich (vergl. Abs. 4.2).

\section{Rißausbreitung im Einkristall}

Ob langsame subkritische Rißerweiterung nur für polykristalline Gesteine oder auch für deren Ausgangsmaterial vorliegt, wurde in Experimenten an Einkristallen untersucht. Dazu wurden in Quarz-Einkristallen Rißlaufereignisse initiiert. Die Bedingungen waren identisch den im vorangehenden Abschnitt beschriebenen: Es wurden Proben mit rechteckigem Querschnitt in Drei-Punkt-Biege-Anordnung unter Vorgabe einer konstanten Rißöffnungsrate $\left(10^{-7} \mathrm{~m} / \mathrm{s}\right)$ getestet. Als Initiierungsstellen wurden Starterkerben (parallel zur c-Achse) mit einem Krümmungsradius von 0,125 mm verwendet. Das Versagen der Proben war von deutlicher Schallemission begleitet.

Die Bruchausbreitungsgeschwindigkeit wurde anhand von Wallnerlinien auf den Bruchflächen abgeschätzt. Dies ist in Abb.53 für den Ausschnitt aus einer repräsenta-

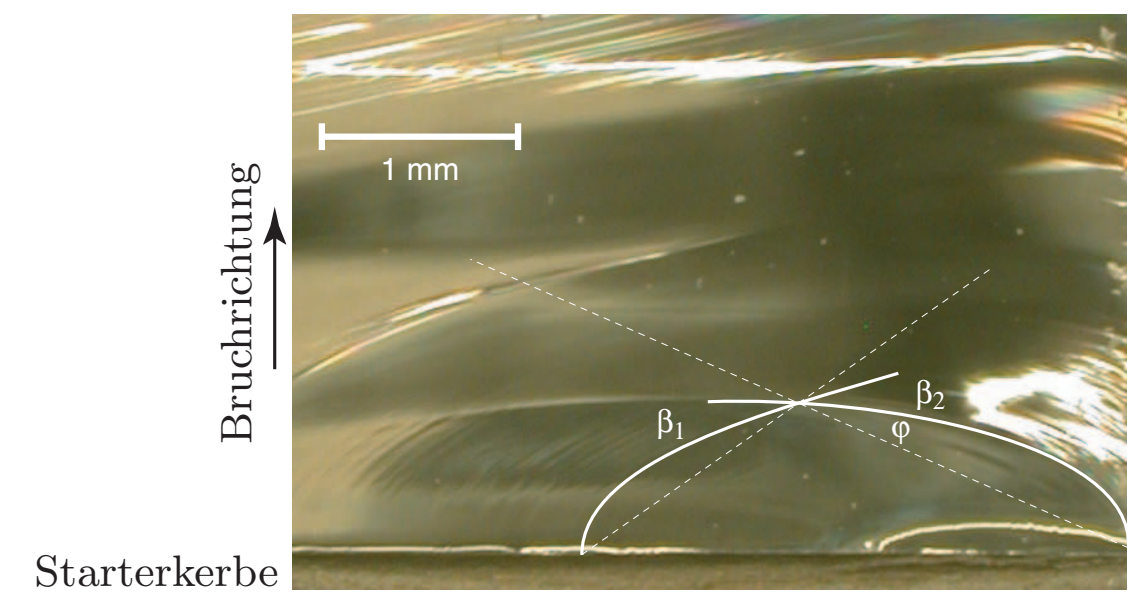

Abb. 53: Bruchfläche eines Quarz-Einkristalles einschließlich der Konstruktionskurven zur Auswertung der Bruchgeschwindigkeit anhand von Wallnerlinien in einem Linienschnittpunkt. 
tiven Bruchfläche gezeigt (vergl. auch Titelbild von [179]). Die Auswertung einer Serie von Experimenten liefert ausschließlich hohe Bruchgeschwindigkeiten. Dabei nimmt die Bruchgeschwindigkeit im Verlaufe des Ausbreitungsvorganges ab. Als niedrigste Rißlaufgeschwindigkeit wurden Werte von $v_{c}=650 \mathrm{~m} / \mathrm{s}$ bestimmt, die höchsten Rißlaufgeschwindigkeiten lagen im Bereich von $v_{c}=2000 \mathrm{~m} / \mathrm{s}$ und waren somit etwa halb so groß wie die Scherwellengeschwindigkeit $\left(v_{s}=4090 \mathrm{~m} / \mathrm{s}\right)$. Zusammenfassend wird festgestellt, daß subkritische Rißerweiterung in Quarz-Einkristallen nicht beobachtet wurde.

\section{Erzeugung von Scherbruchausbreitung}

Die LECEI-Methode der dynamischen Scher(Modus-II)-Beanspruchung wurde, über die Verwendung zur Untersuchung schneller Zug(Modus-I)-Rißausbreitung hinaus, für Experimente zur Erzeugung von Scherrissen eingesetzt, die sich in der Belastungsrichtung ausbreiten (vergl. Abs. 2.4). Dazu wurden die Versuchsbedingungen verändert: Einerseits wurden die Beanspruchungsbedingungen variiert, indem zusätzlich zu der Scher(Modus-II)-Belastung ein Manteldruck auf dem Ligament oder durch Rißschließung ein negativer Modus-I-Spannungsintensitätsfaktor aufgebracht wurde. Des weiteren wurde die Belastungsrate variiert. Andererseits wurden in den verwendeten Proben Schwächezonen in Form von Seitenkerben entlang des avisierten Rißpfades eingebracht. Die Untersuchungen wurde zudem auf geologische Materialien mit geringerer Festigkeit ausgeweitet. Scherbruchausbreitung bzw. eine Änderung des Versagensmodus, wie er beispielsweise in verschiedenen Metallen beobachtet wird, trat jedoch bei sämtlichen Experimenten an Gestein, einschließlich solcher unter quasistatischen Belastungsbedingungen, nicht auf. Rißausbreitung in Richtung der Belastung wurde lediglich in den Experimenten erzielt, die unter Verwendung von Seitenkerben durchgeführt wurden. Allerdings erfolgte die Rißausbreitung in Form von Zugrissen, die, eingefangen in der Schwächezone, unter ständigem Wechsel der Ausbreitungsrichtung in dem Material zwischen den Seitenkerben im Winkel von $70^{\circ}$ auf- und abwärts laufend eine sägezahnförmige Bruchfläche erzeugten. Eine anhand der erzielten Ergebnisse durchgeführte Analyse [180] ergab, daß zur Erzeugung von Scherbruchausbreitung deutlich höhere Spannungsintensitäten als für die Zugrißausbreitung benötigt werden könnten (siehe auch [181]), als unter den vorliegenden Bedingungen und mit den mikrostrukturellen Merkmalen der verwendeten Materialien zu erzielen sind (vergl. [182-184]). Die Ergebnisse stimmen des weiteren mit Modellvorstellungen zum ,strain-softening“-Versagensverhalten geologischer Materialien und den entsprechenden inelastischen Vorgängen innerhalb der Prozeßzone überein [185, 186]. 


\section{Diskussion}

Die in den Experimenten gefundene Rißlaufgeschwindigkeits-Rißlaufzähigkeits-Beziehung $K_{I D}\left(v_{c}\right)$ in Gestein hat den allgemeinen Verlauf, der in Abb. 54 abgebildet ist. Die Kurve teilt sich in drei Geschwindigkeitsbereiche auf, in denen die Höhe der sich einstellenden Rißlaufzähigkeiten bzw. Bruchenergien durch unterschiedliche Mechanismen dominiert wird. Die Ergebnisse weisen im Bereich niedriger Ausbreitungsgeschwindigkeiten eine mit steigender Rißlaufgeschwindigkeit stark ansteigende Rißwiderstandskurve und damit einen $R$-Kurven-Charakter auf, wie er gewöhnlich bei duktilem Rißausbreitungsverhalten beobachtet wird. Für die mit der steigenden Rißlaufgeschwindigkeit einhergehende Rißverlängerung wurden solche steigenden $R$-Kurven in bruchmechanischen Untersuchungen an verschiedenen Gesteinen bereits früh bestimmt und die Bedeutung der getrennten Betrachtung von Rißinitiierung und -ausbreitung erkannt [105, 106,187]. Nach der Implementierung des Meßstandards zur Bestimmung des Initiierungskennwertes wurde dies jedoch lediglich in wenigen Fällen weiterverfolgt [112,188, 189]. Dieser Effekt basiert darauf, wie in Abs. 2.2 beschrieben, daß sich im Verlauf subkritischer Rißausbreitung an der Rißspitze Prozeßzonen zunehmender Größe ausbilden und dadurch ein Anstieg der Energiedissipation resultiert. Die bei zunehmender Bruchgeschwindigkeit steigende Rißwiderstandskurve erklärt das besondere Ausbreitungsverhalten von Rissen in Ge-

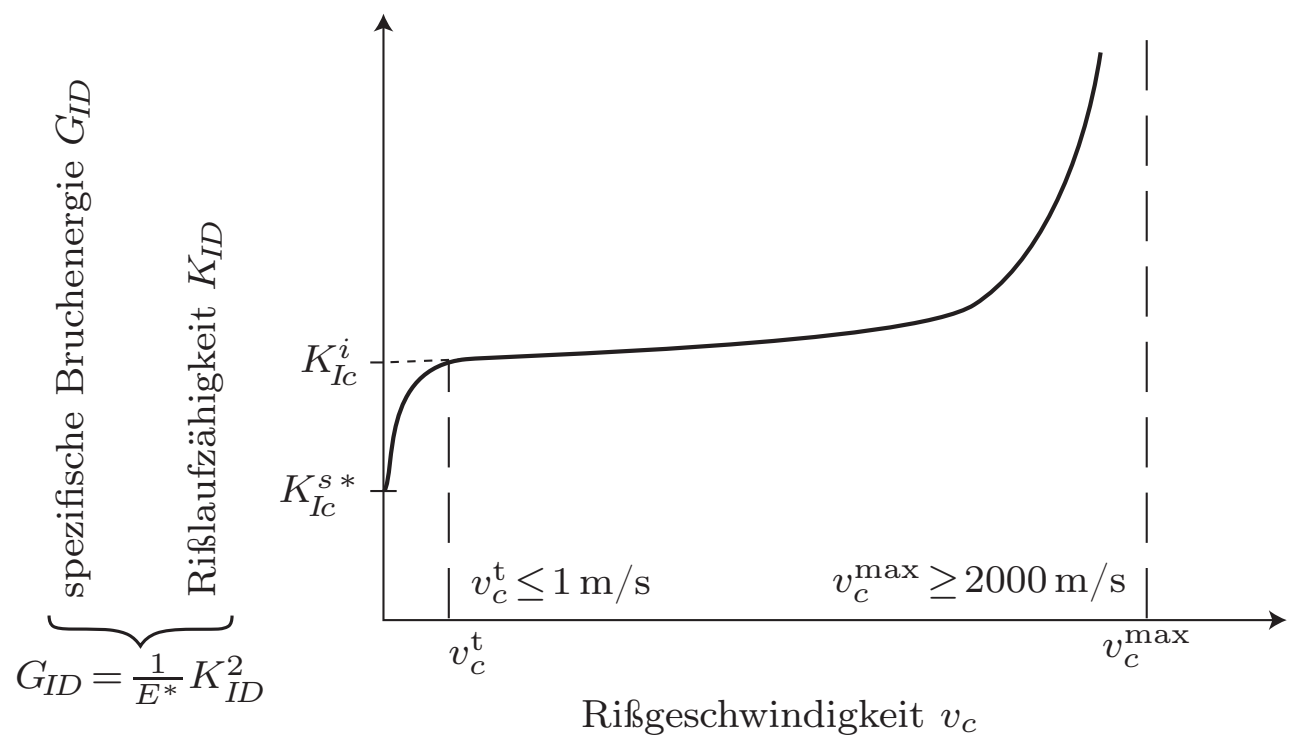

Abb. 54: Experimentell bestimmtes, prinzipielles Verhältnis zwischen Bruchzähigkeit/Bruchenergie und der Ausbreitungsgeschwindigkeit laufender Risse in Gestein, schematisch. 
stein und bestätigt die aufgrund dessen gezogenen Spekulationen (vergl. Abs. 4.2). Dieses Verhalten ist des weiteren ursächlicher Grund für die Möglichkeit der definierten Erzeugung langsamer, stabiler Rißausbreitung in Gestein durch weggeregelte Belastungssysteme trotz des vorliegenden Sprödbruchcharakters. Daß dieses Verhalten auch bei Scherbrüchen beobachtet wird [190] zeigt die universelle Bedeutung der hier gewonnen Erkenntnisse und die Übertragbarkeit auf andere Bruchmoden. Dies gilt möglicherweise ebenso für schnelle Bruchausbreitung, für die bei der untersuchten Zug(Modus-I)-Ausbreitung bei der weiteren Rißbeschleunigung und dem Einsetzen instabilen Rißwachstums die Rißwiderstandskurve zunächst verflacht und in einen Bereich übergeht, für den die gemessenen Rißlaufzähigkeiten keine Abhängigkeit von der Rißlaufgeschwindigkeit aufweisen. Eine Reduktion der Rißlaufzähigkeiten in diesem Geschwindigkeitsbereich, zurückzuführen auf versprödende Effekte durch steigende Dehnraten (vergl. Abs. 2.3.4, Abb. 18), ist nicht zu beobachten. Damit zeigt die Meßkurve in diesem Bereich ein Bruchverhalten, wie es für sprödbrechende Materialien erwartet wird (Abb.19). Gleichsam entspricht der starke Anstieg im Bereich hoher Rißlaufgeschwindigkeiten bis zum Erreichen der maximalen Bruchgeschwindigkeit bei Einsetzen von Bruchgabelung, verursacht durch materialabhängige transiente Effekte im Spannungs- und Temperaturfeld an der Rißspitze (siehe Abs. 2.3.4), dem avisierten Verlauf. Form und Höhe des Anstiegs stimmen mit den, auf der Grundlage unterschiedlicher experimenteller Methoden bestimmten, Ergebnissen anderer sprödbrechender Materialien überein (siehe [191]). Vergleichbare Labordaten an Gesteinen liegen bislang nicht vor. Für geologische Feldbeispiele wird das Vorliegen dynamischer Rißausbreitungsphänomene, auch für Scherbruchausbreitung, bereits diskutiert [129, 130, 192-194].

Das Auftreten stabiler subkritischer Rißerweiterung impliziert, wie auch beobachtet, daß die Bruchzähigkeitswerte $K_{I c}^{s}$, bei denen subkritische Rißlaufereignissse initiieren, einer starken Streuung unterliegen. Durch das Vorliegen des R-Kurvencharakters stellen diese Initiierungszähigkeiten keinen charakteristischen Materialkennwert dar (siehe Abs. 2.2). Ein Initiierungskennwert $K_{I c}^{i}$, der im Sinne der ursprünglichen Definition den Rißwiderstand bei überkritischer Rißausbreitung angibt, läßt sich durch Extrapolation der Meßkurve zu $v_{c}=0$ ermitteln (Abb. 54). Für Solnhofener Kalkstein ergibt sich so ein Initiierungskennwert von $K_{I c}^{i}=1,55 \mathrm{MN} / \mathrm{m}^{3 / 2}$ und für Arkansas Novaculit ist $K_{I c}^{i}=3,00 \mathrm{MN} / \mathrm{m}^{3 / 2}$. Diese Werte liegen in dem Bereich der Initiierungszähigkeiten $K_{I d}^{*}$, die unter dynamischen Beanspruchungsbedingungen erzielt werden (vergl. Abs. 4.5). Ein ähnlicher Anstieg wurde auch für das dynamische Festigkeitsverhalten von Gesteinen festgestellt [195,196]. Ursachen, die zum Anstieg der Initiierungszähigkeiten bei Gesteinen unter dynamischer Beanspruchungsbedingungen führen, wurde aufgrund des Schwerpunktes der Arbeit auf der Beobachtung von Rißausbreitung nicht nachgegangen (vergl. Abs. 2.3.3). Wegen der Verwendung stumpfer Initiierungsstellen ist es nicht möglich über den festgestellten Trend des ansteigenden Verhaltens hinaus quantitative Aussagen über das allgemeine dynamische Initiierungsverhalten von Rissen in Gesteinen aus diesen Beobabachtungen abzulei- 
ten [1]. Dieser Effekt sollte durch weitere gezielte Experimente, auch für scharfe Risse, untersucht werden, zumal für viele Metalle ein gegenteiliges Verhalten beobachtet wird [84].

Insgesamt wurden in beiden Gesteinen für laufende Risse Bruchzähigkeiten $K_{I D}$ gemessen, die die Initiierungszähigkeit $K_{I c}^{s}$ bei Einsetzen stabilen subkritischen Rißwachstums wesentlich übersteigen. Der genaue Vergleich liefert einen Faktor von $K_{I D} / K_{I c}^{s *}=4,5$ für Solnhofener Kalkstein und für Arkansas Novaculit ist $K_{I D} / K_{I c}^{s *}=$ 4,9 (die für den Vergleich herangezogenen Werte für $K_{I c}^{s *}-$ bzw. $G_{I c}^{s *}$, s. u. - beziehen sich jeweils auf die in Abs. 4.5 für $K_{I c}^{s *}$ angegebenen Mittelwerte). Bei der Bestimmung der Bruchenergien $G_{I D}$ aus Rißlaufzähigkeiten $K_{I D}$ muß, bei Rißausbreitungsgeschwindigkeiten bis in den Bereich von $70 \%$ der Rayleighwellengeschwindigkeit, eine Geschwindigkeitskorrektur nach Gl. (30) erfolgen. Für die Bruchenergie ergibt sich so eine Steigerung beim Vergleich der maximalen spezifischen Bruchenergie für laufende Risse $G_{I D}$ mit den entsprechenden quasistatischen Initiierungswerten $G_{I c}^{s *}$ (Abb. 55) Faktoren von $G_{I D} / G_{I c}^{s *}=30$ für Solnhofener Kalkstein bzw. von $G_{I D} / G_{I c}^{s *}=35$ für Arkansas Novaculit. Dabei sei erwähnt, daß schon für Rißlaufereignisse weit unterhalb der maximalen Rißlaufgeschwindigkeiten die Rißlaufenergien die Energien bei quasistatischer Initiierung in beiden Gesteinen signifikant, d. h. um einen Faktor fünf über-

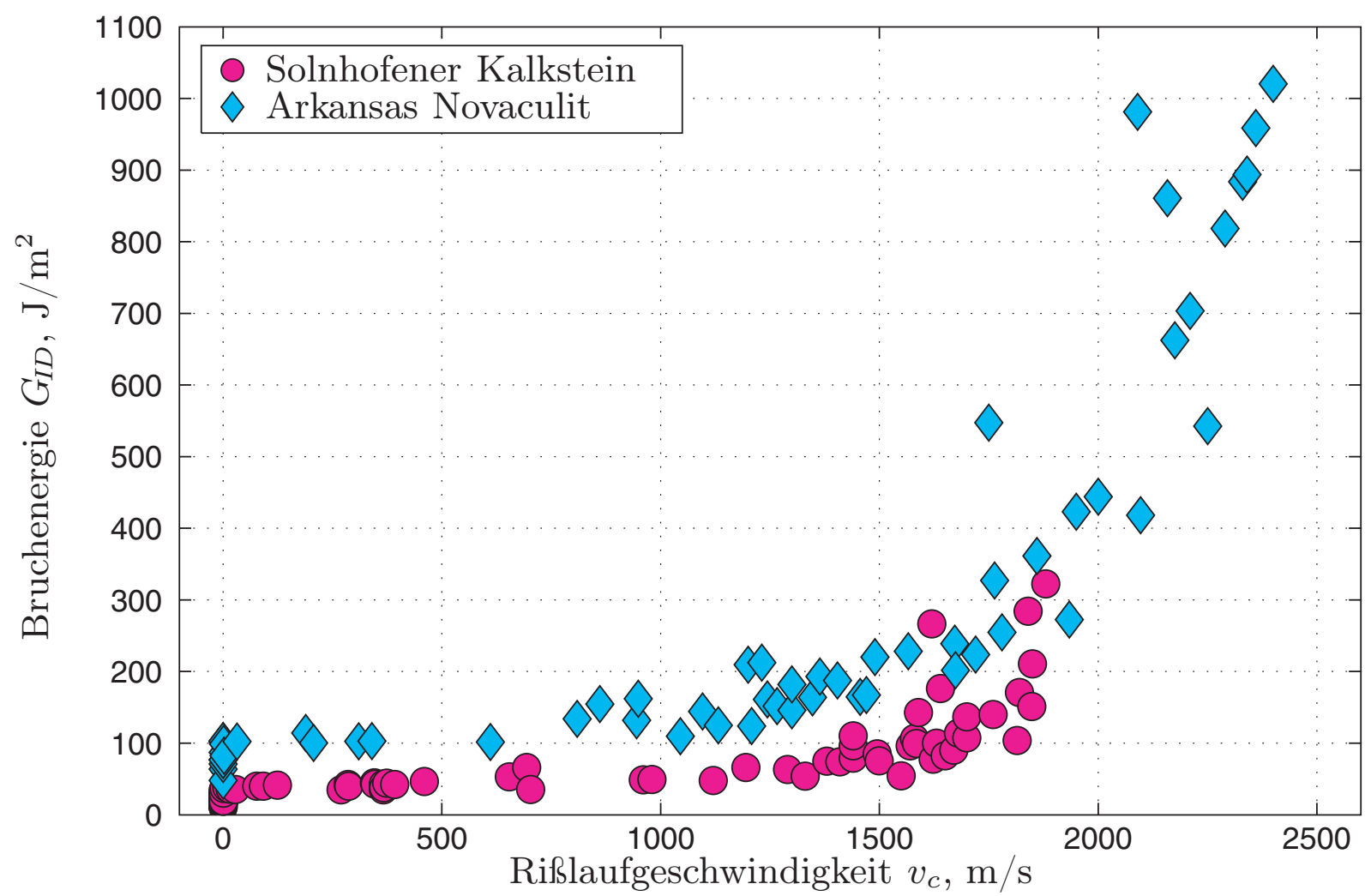

Abb. 55: Bruchenergien laufender Risse in Arkansas Novaculit und Solnhofener Kalkstein. 
steigen. Diese Ergebnisse ermöglichen ein verbessertes Verständnis der Energiebilanz von dynamischen, überkritischen Bruchvorgängen in der Erde. Da solchen Bruchvorgängen Rißlaufereignisse hoher Geschwindigkeiten zugrunde liegen, wird durch Modellvorstellungen auf der Basis von Rißinitiierungsdaten aus Standardlaborversuchen die tatsächliche Energiedissipation unterschätzt. Die Berücksichtigung dynamischer Zug(Modus-I)-Rißausbreitung bis in den Bereich hoher Rißlaufgeschwindigkeiten erklärt allerdings nicht den gesamten Unterschied, der zwischen Laborexperimenten und in-situ-Bruchenergien festgestellt wird (siehe Abs. 2.4). Insgesamt resultiert aus dem o. a. Vergleich eine Steigung der Bruchenergie um etwa zwei Größenordnungen, wenn statt der verwendeten Mittelwerte jeweils die niedrigste der ermittelten Initiierungszähigkeiten angesetzt und berücksichtigt wird, daß die Initiierungszähigkeiten nicht von scharfen Rissen gewonnen wurden. Die Wirkung von, in der Erdkruste vorhandenen, Formationsfluiden sowie zeitabhängigen Effekten, bei üblicherweise gegenüber Laborexperimenten niedrigeren geologischen Belastungsraten, würden diese Initiierungswerte noch weiter reduzieren [162,197].

Dazu ist anzumerken, daß sich aus bruchmechanischen Betrachtungen eine Erhöhung der Energiedissipation von Rißlaufereignissen gegenüber den hier durchgeführten Experimenten unter Berücksichtigung sonstiger in-situ-Umgebungsbedindungen ergibt, wie etwa der Einflüsse des Manteldrucks [181, 184, 198]. Gleiches gilt für Scherbruchphänomene, für die sich eine bruchmechanische Betrachtungsweise noch nicht durchgesetzt hat und dynamische Einflüsse häufig nicht berücksichtigt werden [132,199-202]. Ebenso stellen sich für andere, weniger homogene und feinkörnige geologische Materialien höhere Energiedissipationswerte ein als bei den hier untersuchten Gesteinen [160,163,203]. Die mineralogische Zusammensetzung der Erdkruste wird von dem monomineralischen Quarzit Arkansas Novaculit eher repräsentiert als von dem Calcit-dominierten Solnhofer Kalkstein. Es wird daher davon ausgegangen, dass die Ergebnisse der Bruchexperimente an Arkansas Novaculit relevanter bei der Übertragung auf tektonische Prozesse im Krustenmaßstab sind. Es wird jedoch darauf verwiesen, daß in vielen tektonisch aktiven Gebieten der Erde Karbonatgesteine weit verbreitet sind (z. B. Griechenland). Des weiteren nehmen Karbonatgesteine als Erdölmutter- und Speichergesteine eine wirtschaftlich wichtige Stellung ein.

Die hier berichteten Ergebnisse liefern wichtige Erkenntnisse im Zusammenhang mit der Ausbreitung von Zugrissen in der Erde [194,204-206]. Weitere Anwendungsgebiete ergeben sich auch für geotechnische Fragestellungen z. B. bei der dynamischen Initiierung und Ausbreitung von künstlich erzeugten hydraulischen Zugrissen im Zusammenhang mit der Injektion von Fluiden zur Stimulation von Kohlenwasserstofflagerstätten sowie bei der hydrothermalen Energiegewinnung oder allgemein für Stabilitätsbetrachtungen geotechnischer Bauwerke wie Tunnel, Kavernen oder Bergwerken. Die im Rahmen dieser Arbeit in Laborexperimenten untersuchten Rißlaufereignisse überdecken den gesamten Bereich möglicher Rißlaufgeschwindigkeiten und bilden so die Bedingungen bezüglich der Ausbreitungsgeschwindigkeiten ab, die sich im Verlauf natürlicher Bruchvorgänge einstellen. Somit werden ratenabhängige Prozesse auf 
eine realistische Weise beschrieben und eines der Skalierungsprobleme überwunden, das beim Vergleich von Labordaten mit Feldbeispielen üblicherweise auftritt. Es wird angenommen, daß die im Rahmen dieser Arbeit erzielten Ergebnisse zur geschwindigkeitsabhängigen Energiedissipation laufender Risse prinzipiell auch auf Scherversagensfälle übertragbar und somit als neue Eingangsparameter für Modellvorstellungen zur Interpretation von Bruchvorgängen bei Erdbeben geeignet sind [133]. Dies sollte durch weitere Untersuchungen zur Erzeugung von Scher(Modus-II)-Versagen, unter Beobachtung mikromechanischer Prozesse, in dynamischen Laborexperimenten weiter verfolgt werden. 


\section{Literaturverzeichnis}

[1] Anderson, T. L.: Fracture Mechanics Fundamentals and Applications. CRC Press, Boca Raton, 1991.

[2] Blauel, J. G., J. F. Kalthoff und E. Sommer: Die Bruchmechanik als Grundlage für das Verständnis des Festigkeitsverhaltens. Materialprüfung, $12(3): 69-76,1970$.

[3] Broberg, K. B.: Cracks and Fracture. Academic Press, San Diego, 1999.

[4] Broek, D.: Elementary Engineering Fracture Mechanics. Martinus Nijhoff Publishers, fourth revised edition, 1986.

[5] Gross, D. und Th. Selig: Bruchmechanik - mit einer Einführung in die Mikromechanik. Springer, Berlin, dritte Auflage, 2001.

[6] Hahn, H. G.: Bruchmechanik: Einführung in die theoretischen Grundlagen. Leitfäden der angewandten Mathematik und Mechanik, Bd. 30. Teubner, 1976.

[7] Kalthoff, J. F.: Vorlesung Bruchmechanik - Sicherheitsanalysen I. RuhrUniversität Bochum, WS 2000.

[8] Lawn, B. R.: Fracture of Brittle Solids. Cambridge University Press, Cambridge, second edition, 1993.

[9] Westergand, H. M.: Bearing Pressures and Cracks. Journ. of Applied Mechanics, 6:49-53, 1939.

[10] Sneddon, I. N.: The Distribution of Stress in the Neighborhood of a Crack in an Elastic Solid. Proceedings, Royal Society of London, A-187:229-260, 1946.

[11] Irwin, G. R.: Analysis of Stresses and Strains Near the End of a Crack Traversing a Plate. J. Appl. Mech., 24:361-364, 1957.

[12] Williams, M. L.: On the Stress Distribution at the Base of a Stationary Crack. Journ. of Applied Mechanics, 24:109-114, 1957.

[13] SiH, G. C. (editor): Methods of Analysis and Solutions of Crack Problems, Mechanics of Fracture 1, Leyden, 1973. Noordhoff International Publishing.

[14] Kanninen, M. F. and C. H. Popelar: Advanced Fracture Mechanics, volume 15 of Oxford Engineering Science Series. Oxford University Press, New York, Clarendon Press, Oxford, 1985. 
[15] Creager, M. and P. C. PARis: Elastic Field Equations for Blunt Cracks with Reference to Stress Corrosion Cracking. International Journal of Fracture Mechanics, 3:247-252, 1967.

[16] NeuBer, H.: Kerbspannungslehre. Springer, Berlin, 1937.

[17] Peterson, R. E.: Stress Concentration Factors. John Wiley \& Sons, NY, 1940.

[18] Williams, M. L.: The Bending Stress Distribution at the Base of a Stationary Crack. Journ. of Applied Mechanics, 28:78-82, 1961.

[19] Rice, J. R.: Limitations to the Small Scale Yielding Approximation for Crack Tip Plasticity. Journal of the Mechanics and Physics of Solids, 22:17-26, 1974.

[20] Sin, G. C.: On the Westergaard Method of Crack Analysis. International Journal of Fracture Mechanics, 2:628-631, 1966.

[21] Cotterell, B.: Notes on the Paths and Stability of Cracks. International Journal of Fracture Mechanics, 2(3):526-533, 1966.

[22] Dally, J. W. and W. R. Riley: Experimental Stress Analysis. McGraw-Hill, New York, $3^{\text {rd }}$ edition, 1991.

[23] Sanford, R. J., W. L. Fourney, R. Chona and G. R. Irwin: A Photoelastic Study of the Influence of Non-Singular Stresses in Fracture Test Specimens. Technical Report, University of Maryland, MD, Contr. No. NUREG/CR-2179 \& ORNL/Sub-7778/2, available from GPO Sales Program, Division of Technical Information and Document Control, U.S.-NRC, Washington D.C., 1981.

[24] TAda, H., P. C. PARIS and G. R. IRwin: The Stress Analysis of Cracks Handbook. Paris Productions Inc., St. Louis, second edition, 1985.

[25] Theilig, H. und J. Nickel: Spannungsintesitätsfaktoren. VEB, Leipzig, 1987.

[26] Murakami, Y.: Stress Intensity Faktors Handbook. Pergamon Press, Oxford, 1987.

[27] IRwin, G. R.: Plastic zone near a crack and fracture toughness. In Proceedings of the 7th Sagamore Ordnance Materials Research Conference on Mechanics \& Metals Behavior of Sheet Material, Raquette Lake, NY, August 1960, volume 4, pages 463-478. Syracuse University Press, 1960.

[28] Atkinson, B. K.: Introduction to Fracture Mechanics and its Geohysical Applications. In AtKinson, B. K. (editor): Fracture Mechanics of Rock, chapter 1, pages 1-26. Academic Press, London, 1987. 
[29] Schmidt, R. A.: A Microcrack Model and its Significance to Hydraulic Fracturing and Fracture Toughness Testing. In Summers, D. A. (editor): Proceedings of the 21 $1^{\text {st }}$ U.S. Symposium on Rock Mechanics, May 28-30, 1980, Univ. Missouri, pages 581-590, 1980.

[30] Hoagland, R. G., G. T. Hahn and A. R. Rosenfield: Influence of microstructure on fracture propagation in rock. Rock Mechanics, 5(2):77-106, 1973.

[31] Hiese, W.: Gültigkeitskriterien zur Bestimmung von Scherbruchzähigkeiten. Doktorarbeit, Ruhr-Universität Bochum, Fakultät für Maschinenbau, Januar 2000 .

[32] Fernández Zúñiga, D., J. F. Kalthoff, A. Fernández Canteli, J. Grasa and M. Doblaré: Three dimensional effects along the crack front influencing the validity criteria for determining fracture toughness. ICF XI, $11^{\text {th }}$ International Conference on Fracture, Turin, March 20-25, 2005, Paper No. 4087, 2005.

[33] Curran, D. R., L. Seaman and D. A. Shockey: Dynamic Failure of Solids. Physics Reports, 147(5\&6):253-388, 1987.

[34] Swanson, P. L.: Tensile Fracture Resistance Mechanisms in Brittle Polychrystals: An Ultrasonic and in situ Microscopy Investigation. J. Geophys. Res., 92(B8):8015-8036, 1987.

[35] ASTM E 399-06 ${ }^{\epsilon 1}$ : Standard Test Method for Linear-Elastic Plane-Strain Fracture Toughness $K_{\text {Ic }}$ of Metallic Materials. Annual Book of ASTM Standards 03.01 Metals - Mechanical Testing, American Society for Testing and Materials, West Conshohocken, 2007.

[36] SÄHn, S. und H. GÖLDnER: Bruch- und Beurteilungskriterien in der Festigkeitslehre. Fachbuchverlag, Leipzig, Köln, 2. Auflage, 1993.

[37] FKM-RichtLinie: Bruchmechanischer Festigkeitsnachweis für Maschinenbauteile. 3. Ausgabe, Forschungskuratorium für den Maschinenbau, VDMA Verlag GmbH, Frankfurt, 2006.

[38] Richard, H. A.: Festigkeitsnachweis unter Mixed-Mode-Beanspruchung. Materialprüfung, 45(11-12):513-518, 2003.

[39] Griffith, A. A.: The Phenomena of Rupture and Flow in Solids. Philosophical Transactions of The Royal Society (reprint, commented by Gilman, J. J.), 221A:163-198, 1920. 
[40] Griffith, A. A.: The Theory of Rupture. In Biezeno, C. B. and J. M. Burgers (editors): Proceedings of the First International Congress for Applied Mechanics, Delft, Netherlands, April 22-26, 1924, pages 55-63, Delft, 1925. Waltman Jr., J.

[41] Irwin, G. R.: Fracture. In FlüGGe, S. (editor): Handbuch der Physik, volume 6, pages 551-590. Springer, Berlin, Göttingen, Heidelberg, 1958.

[42] Irwin, G. R.: Fracture Dynamics. In Fracturing of Metals, pages 147-166. ASM, Cleveland, OH, 1948.

[43] Orowan, E.: Fracture and strength of solids. Reports on Progress in Physics, 12:185-232, 1949.

[44] Irwin, G. R. and J. A. Kies: Fracturing and Fracture Dynamics. The Welding Journal Res. Suppl., 31(2):95-6-100-5, 1952.

[45] Kalthoff, J. F.: Zur Ausbreitung und Arretierung schnell laufender Risse. In: Fortschrittsberichte der VDI-Zeitschriften, Band 4 der Reihe 18, Bruchvorgänge und Schadensanalyse. VDI-Verlag, Düsseldorf, Dezember 1978.

[46] Schwalbe, K.-H.: Mechanik und Mechanismen des stabilen Rißwachstums in metalllischen Werkstoffen. In: Fortschrittsberichte der VDI-Zeitschriften, Band 3 der Reihe 18, Bruchvorgänge und Schadensanalyse. VDI-Verlag, Düsseldorf, Oktober 1977.

[47] Ravi-Chandar, K., J. Lu, B. Yang and Z. Zhu: Failure Mode Transitions in Polymers under High Strain Rate Loading. International Journal of Fracture, 101:33-72, 2000.

[48] Chaо, Y-J and X-K Zhu: A Simple Theory for Describing the Transition between Tensile and Shear Mechanisms in Mode I, II, III, and Mixed-Mode Fracture. In Miller, K. J. and D. L. McDowell (editors): Mixed-Mode Crack Behavior, volume ASTM STP 1359, pages 41-57, West Conshohocken, PA, 1999. American Society for Testing and Materials.

[49] Podleschny, R.: Untersuchung zum Instabilitätsverhalten scherbeanspruchter Risse. Doktorarbeit, Ruhr-Universität Bochum, Fakultät für Maschinenbau, Bochum, April 1995.

[50] Horit, H. and S. Nemat-Nasser: Brittle Failure in Compression: Splitting, Faulting and Brittle-Ductile Transition. Phil. Trans. R. Soc. Lond., A 319:337-374, 1986.

[51] Richard, H. A.: Bruchvorhersagen bei überlagerter Normal- und Schubbeanspruchung sowie reiner Schubbelastung von Rissen. Habilitationsschrift, Universität Kaiserslautern, Fachbereich Maschinenwesen, 1984. 
[52] Erdogan, F. and G. C. Sin: On the Crack Extension in Plates under Plane Loading Conditions and Transverse Shear. Journal of Basic Engineering, 85:519-527, 1963.

[53] Blázquez, A., J. F. Kalthoff, I. Palomino and A. Fernández Canteli: Numerical Calculations of the Singular Stress Fields of Failing Mode-II Cracks in Comparison to Experiments. In ICCES 04, International Conference on Computational \& Experimental Engineering 85 Sciences, Madeira, July 26-29, 2004, 2004.

[54] Kalthoff, J. F., A. Fernández Canteli, A. Blázquez and D. FernánDEZ Zúñiga: Singular Stress Fields and Instability Conditions for Mode-II and Mixed-Mode loaded Cracks. Strength, Fracture and Complexity, 4(3):141-160, 2006.

[55] Cotterell, B. and J. R. Rice: Slightly Curved or Kinked Cracks. International Journal of Fracture, 16(2):155-169, 1980.

[56] Bergmannshoff, D.: Das Instabilitätsverhalten zug-/scherbeanspruchter Risse bei Variation des Belastungspfades. Doktorarbeit, Ruhr-Universität Bochum, Fakultät für Maschinenbau, 2007.

[57] Мотт, N. F.: Fracture of Metals: Theoretical Considerations. Engineering, 165:16-18, 1948.

[58] Yoffe, E. H.: The Moving Griffith Crack. Philosophical Magazine, 42:739750, 1951.

[59] Roberts, D. K. and A. A. Wells: The Velocity of Brittle Fracture. Engineering, 171:820-821, 1954.

[60] Schardin, H: Velocity effects in fracture. In Averbach, B. L., D. K. FelBeCK, G. T. HAhn and D. A. Thomas (editors): Fracture, pages 297-330. John Wiley, 1959.

[61] Berry, J. P.: Some Kinetic Considerations of the Griffith Criterion For Fracture-I, Equations of Motion at Constant Force. J. Mech. Phys. Solids, 8:194-206, 1960.

[62] Berry, J. P.: Some Kinetic Considerations of the Griffith Criterion For Fracture-II, Equations of Motion at Constant Deformation. J. Mech. Phys. Solids, 8:207-216, 1960.

[63] Broberg, K. B.: The Propagation of a Brittle Crack. Arkiv för Fysik, 18:159192, 1960. 
[64] Erdogan, F.: Crack propagation theories. In Liebowitz, H. (editor): Fracture: An Advanced Treatise, Mathematical Fundamentals, volume 2, chapter 5, pages 497-590. Academic Press, San Diego, 1968.

[65] Kalthoff, J. F.: On the Characteristic Angle for Crack Branching in Brittle Materials. International Journal of Fracture Mechanics, 7:478-480, 1971.

[66] Freund, L. B.: Crack propagation in an elastic solid subjected to general loading-I, Constant rate of extension. Journal of the Mechanics and Physics of Solids, 20:129-140, 1972.

[67] Freund, L. B.: Crack propagation in an elastic solid subjected to general loading-II, Non-uniform rate of extension. Journal of the Mechanics and Physics of Solids, 20:141-152, 1972.

[68] Freund, L. B.: Crack propagation in an elastic solid subjected to general loading-III, Stress Wave Loading. Journal of the Mechanics and Physics of Solids, 21:47-61, 1973.

[69] Freund, L. B.: The Analysis of Elastodynamic Crack Tip Stress Fields. In Nehmat-Nasser, S. (editor): Mechanics Today, volume 3, pages 55-91. Pergamon, 1976.

[70] Achenbach, J. D.: Dynamic Effects in Brittle Fracture. In Nehmat-Nasser, S. (editor): Mechanics Today, volume 1, 1972, pages 1-57. Pergamon Press, Oxford, 1974.

[71] Gross, D.: Beiträge zu dynamischen Problemen in der Bruchmechanik. Habilitationsschrift, Universität Stuttgart, 1974.

[72] Sin, G. C.: Some Elastodynamic Problems of Cracks. International Journal of Fracture Mechanics, 4:51-68, 1968.

[73] Kerkhoff, F.: Bruchvorgänge in Gläsern. Verlag der Deutschen Glastechn. Ges., Frankfurt a. M., 1970.

[74] Kalthoff, J. F.: On the measurement of dynamic fracture toughnesses a review of recent work. Int. Journ. of Fracture, 27:277-298, 1985.

[75] Washabaugh, P. D. and W. G. Knauss: A Reconciliation of Dynamic Crack Velocity and Rayleigh Wave Speed in Isotropic Brittle Solids. International Journal of Fracture, 65:97-114, 1994.

[76] Freund, L. B.: Dynamic Fracture Mechanics. Cambridge University Press, 1990. 
[77] Kalthoff, J. F.: Dynamic Fracture Toughnesses. In Bushov, K. H. J., R. W. Cahn, M. C. Flemings, B. Ilschner, E. S. Kramer and S. MahaJAN (editors): Encyclopedia of Materials - Science and Technology. Pergamon, Oxford, 2001.

[78] Ireland, D. R.: Critical Review of Instrumented Impact Testing. In International Conference on Dynamic Fracture Toughness, London, 5-7 July 1976, pages 113-125. Welding Institute, Cambridge, 1977.

[79] Kalthoff, J. F. and D. A. Shockey: Instability of Cracks under Impulse Loads. Journ. of Applied Physics, 48(3):986-993, 1977.

[80] Kalthoff, J. F., S. Winkler and J. Beinert: The Influence of Dynamic Effects in Impact Testing. Int. Journ. of Fracture, 13:528-531, 1977.

[81] Böнme, W.: Experimentelle Untersuchungen dynamischer Effekte beim Kerbschlagbiegeversuch. Doktorarbeit, TU Darmstadt, FB Mechanik, 1985.

[82] Kalthoff, J. F.: The Concept of Impact Response Curves. In Metals Handbook, volume 8 of Mechanical Testing, pages 269-273. American Society for Metals, Metals Park, Ohio, 1985.

[83] Nakamura, T., C. F. Shin and L. B. Freund: Analysis of a Dynamically Loaded Three-Point-Bend Ductile Fracture Specimen. Engng. Fracture Mech., 25:323-339, 1986.

[84] Kalthoff, J. F.: Experimental Fracture Dynamics. In Klepaczko, J. R. (editor): CISM Courses and Lectures No. 310, Crack Dynamics in Metallic Materials, International Centre for Mechanical Sciences, Udine, Italy, pages 75-253, Wien - New York, 1990. Springer-Verlag.

[85] Achenbach, J. D.: Wave Propagation in Elastic Solids. North-Holland Publishing Company, Amsterdam - London, 1973.

[86] Pandorf, T.: Experimentelle und Numerische Untersuchungen zur Kerbspitzenbelastung bei schlagbelasteten Biegeproben. Doktorarbeit, Ruhr-Universität Bochum, Fakultät für Maschinenbau, Bochum, August 1998.

[87] Kalthoff, J. F.: Shadow Optical Method of Caustics. In Kobayashi, A. S. (editor): Handbook on Experimental Mechanics, chapter 9, pages 407-476. VCH Publishers, Inc., New York, second edition, 1993.

[88] Kalthoff, J. F.: Persönliche Mitteilung. Bochum, 2006.

[89] KalthofF, J. F.: On some current problems in experimental fracture mechanics. In Knauss, W. G., K. Ravi-Chandar and A. J. Rosakis (editors): Workshop on Dynamic Fracture, California Institute of Technology, Pasadena, February 17-18, 1983, pages 11-35, 1983. 
[90] Broberg, K. B.: How Fast Can a Crack Go? Materials Science, 32(1):80-86, 1996.

[91] Ravi-Chandar, K.: An Experimental Investigation into Dynamic Fracture: II. Microstructural Aspects. International Journal of Fracture, 26:65-80, 1984.

[92] Ravi-Chandar, K.: An Experimental Investigation into Dynamic Fracture: III. On Steady-State Crack Propagation and Crack Branching. International Journal of Fracture, 26:141-154, 1984.

[93] Irwin, G. R., T. Kobayashi, W. L. Fourney, J. T. Metcalf and J. W. DAlly: Photoelastic Studies of Crack Propagation and Arrest in Polymers and 4340 Steel. Technical Report, University of Maryland, MD, Contr. No. NUREG/CR-0542, available from National Technical Information Service, Springfield, VA, December 1978.

[94] Ravi-Chandar, K.: Dynamic Fracture of Nominally Brittle Materials. International Journal of Fracture, 90:83-102, 1998.

[95] Kalthoff, J. F.: On the Propagation Direction of Bifurcated Cracks. In Sin, G. C. (editor): Proc. Int. Conf. on Dynamic Crack Propagation, Lehigh University, Bethlehem, Pa., July 10-12, 1972, pages 449-458. Nordhoff Int. Publ., 1973.

[96] Irwin, G. R.: Crack Arrest Tests. In Metals Handbook Ninth Edition, volume 8 of Mechanical Testing, pages 284-286. American Society for Metals, Metals Park, Ohio, 1985.

[97] Kalthoff, J. F.: Vorlesung Bruchmechanik - Sicherheitsanalysen II. RuhrUniversität Bochum, SS 2001.

[98] Whittaker, B. N., R. N. Singh and G. Sun: Rock Fracture Mechanics Principles, Design and Applications, volume 72 of Developements of Geotechnical Engineering. Elsevier, Amsterdam - London - New York Tokyo, 1992.

[99] Hoek, E. and Z. T. Bieniawski: Brittle fracture propagation in rock under compression. Int. Journ. of Fracture, 1(3):137-155, 1965.

[100] MÜLLER, W.: Experimentelle und numerische Untersuchungen zur Rißausbreitung im anisotropen Gestein in der Nähe von Grenzflächen. Doktorarbeit, Berichte des Institutes für Geophysik der Ruhr-Universität Bochum, Reihe A, Nr. 22, Bochum, Juni 1987.

[101] Peck, L., R. C. Nolen-Hoeksema, C. C. Barton and R. B. Gordon: Measurement of the resistance of imperfectly elastic rock to the propagation of tensile cracks. J. Geophys. Res., 90(B9):7827-7836, August 1985. 
[102] Bieniawski, Z. T.: The Phenomenon of Terminal Fracture Velocity in Rock. Felsmechanik und Ingenieurgeologie, 6(3):113-125, 1968.

[103] Shockey, D. A., D. R. Curran, L. Seaman, J. T. Rosenberg and C. F. Petersen: Fragmentation of Rock under Dynamic Loads. Int. J. Rock Mech. Min. Sci. \& Geomech. Abstr., 11:303-317, 1974.

[104] Grady, D. E. and M. E. Kipp: Dynamic Rock Fragmentation. In Atkinson, B. K. (editor): Fracture Mechanics of Rock, chapter 10, pages 429-476. Academic Press, London, 1987.

[105] Schmidt, R. A.: Fracture-toughness testing of limestone. Experimental Mechanics, 16(5):161-167, 1976.

[106] Schmidt, R. A. and T. L. Lutz: $K_{I c}$ and $J_{I c}$ of Westerly Granite - Effect of Thickness and In-Plane Dimensions. In Freiman, S. W. (editor): Fracture Mechanics Applied to Brittle Materials, volume ASTM STP 678, pages 166-182, Philadelphia, PA, 1979. American Society for Testing and Materials.

[107] Labuz, J. F., S. P. SHAH and C. H. DOWDING: The Fracture Process Zone in Granite: Evidence and Effect. Int. J. Rock Mech. \& Min. Sci., 24(4):235-246, 1987.

[108] Ouchterlony, F.: A simple R-curve Approach to Fracture Toughness Testing of Rock. In Goodman, R. E. and F. E. Heutze (editors): Issues in rock mechanics, Proceedings of the 23 ${ }^{\text {rd }}$ U.S. Symposium on Rock Mechanics, University of California, Berkeley, California, August 25-27 1982, pages 515-522, New York, 1982. AIME.

[109] International Society for Rock Mechanics: Commission on Testing Methods, Ouchterlony, F. (Co-ordinator), Suggested methods for determining the fracture toughness of rock. Int. J. Rock Mech. Min. Sci. \& Geomech. Abstr., 25(2):7196, 1988.

[110] Ouchterlony, F.: On the background to the formulae and accuracy of rock fracture toughness measurements using ISRM standard core specimens. Int. J. Rock Mech. Min. Sci. \& Geomech. Abstr., 26(1):13-23, 1989.

[111] Rummel, F. und R. Winter: Bestimmung der Bruchzähigkeiten und der spezifischen Oberflächenenergie von Gesteinen im Dreipunkt-Biegeversuch. 4. Teilbericht, BMFT-Forschungsvorhaben ET 3023 - Stimulation gering permeabler tiefer Erdgaslagerstätten, Ruhr-Universität Bochum, AG Geophysikalische Gesteinsuntersuchungen, Januar 1979.

[112] Matsuki, K., S. S. Hasibuan and H. Takahashi: Specimen Size Requirements for Determining the Inherent Fracture Toughness of Rocks According to the ISRM Suggested Methods. Int. J. Rock Mech., 28(5):365-374, 1991. 
[113] Rice, J. R.: The Mechanics of Earthquake Rupture. In Dziewonski, A. M. and E. Boschi (editors): Physics of the Earth's Interior (Proc. International School of Physics 'Enrico Fermi', Course 78, 1979), pages 555-649. Italian Physical Society and North-Holland Publ. Co., 1980.

[114] Rudnicki, J. W.: Fracture Mechanics Applied to the Earth's Crust. Annu. Rev. Earth Planet. Sci., 8:489-525, 1980.

[115] Backers, T., O. Stephansson and E. Rybacki: Rock Fracture Toughness Testing in Mode II - Punch-Through Shear Test. Int. J. Rock Mech. \& Min. Sci., 39(6):755-769, 2002.

[116] Petit, J. P. and M. Barquins: Can Natural Faults Propagate Under Mode II Conditions? Tectonics, 7(6):1243-1256, 1988.

[117] Hubert-Ferrari, A., G. King, I. Manighetti, R. Armijo, B. Meyer and P. TAPPONNIER: Long-term Elasticity in the Continental Lithoshere; Modelling the Aden Ridge Propagation and the Anatolian Extrusion Process. Geophysical Journal International, 153(1):111, 2003.

[118] Reches, Z. and D. A. LockneR: Nucleation and growth of faults in brittle rocks. J. Geophys. Res., 99(B9):18159-18173, September 1994.

[119] TE Kamp, L.: Numerische Modellierung von Rißwechselwirkung. Doktorarbeit, Berichte des Institutes für Geophysik der Ruhr-Universität Bochum, Reihe A, Nr. 49, Bochum, Juli 1996.

[120] Vermilye, J. M. and C. H. Scholz: The Process Zone: A Microstructural View of Fault Growth. J. Geophys. Res., 103(B6):12223-12237, June 1998.

[121] Kemeny, J.: A model for non-linear rock deformation under compression due to sub-critical crack growth. Int. J. Rock Mech. Min. Sci. \& Geomech. Abstr., 28(6):459-467, 1991.

[122] Kanamori, H. and H. H. Heaton: Microscopic and Macroscopic Physics of Earthquakes. In Rundle, J. B., D. L. Turcotte and W. Klein (editors): GeoComplexity and the Physics of Earthquakes, volume 120 of Geophysical Monograph Series, page 1429-1496. AGU, 2000.

[123] Di Toro, G., S. Nielsen and G. Pennacchioni: Earthquake rupture dynamics frozen in exhumed ancient faults. Nature, 436(7053):1009-1012, August 2005.

[124] Scholz, C.: The Mechanics of Earthquakes and Faulting. Cambridge University Press, $2^{\text {nd }}$ edition, 2002. 
[125] Freund, L. B.: The Mechanics of Dynamic Shear Crack Propagation. J. Geophys. Res., 84(B5):2199-2209, 1979.

[126] Das, S.: Application of dynamic shear crack models to the study of the earthquake faulting process. International Journal of Fracture, 27(3-4):263-276, 1985.

[127] Rice, J. R.: New Perspectives on Crack and Fault Dynamics. In Aref, H. and J. W. Phillips (editors): Mechanics for a New Millennium (Proceedings of the 20th International Congress of Theoretical and Applied Mechanics, 27 Aug - 2 Sept 2000, Chicago), pages 1-23. Kluwer Academic Publishers, 2001.

[128] Rice, J. R.: Some Studies of Crack Dynamics. In Bouchaud, E., D. Jeulin, C. Prioul and S. Roux (editors): Physical Aspects of Fracture (Proceedings of NATO Advanced Study Institute on Physical Aspects of Fracture, 5-17 June 2000, Cargèse, Corsica), pages 3-11, Dordrecht, 2001. Kluwer Academic Publishers.

[129] Bhat, H. S., M. Olives, R. Dmowska and J. R. Rice: Role of fault branches in earthquake rupture dynamics. J. Geophys. Res., 112(B11309), 2007. doi: 10.1029/2007JB005027.

[130] NüChter, J.A. and B. StöCKhert: Coupled stress and pore fluid pressure changes in the middle crust: Vein record of coseismic loading and postseismic stress relaxation. Tectonics, 27(TC1007), 2008. doi:10.1029/2007TC002180.

[131] LI, V. C.: Mechanics of Shear Rupture Applied to Earthquake Zones. In Atkinson, B. K. (editor): Fracture Mechanics of Rock, chapter 9, pages 351428. Academic Press, London, 1987.

[132] Rice, J. R., C. G. Sammis and R. Parsons: Off-Fault Secondary Failure Induced by a Dynamic Slip Pulse. Bull Seismol. Soc. Am., 95(1):109-134, 2005.

[133] Rice, J. R. and M. Cocco: Seismic fault rheology and earthquake dynamics. In Handy, M. R., G. Hirth and N. Hovius (editors): Tectonic Faults: Agents of Change on a Dynamic Earth (Dahlem Workshop 95, Berlin, 16-21 January 2005, on The Dynamics of Fault Zones), chapter 5, pages 99-137. The MIT Press, Cambridge, MA, 2007.

[134] DeBel, C. P.: Experimental Evaluation of Brittle Crack Propagation Velocity - an Improved Technique. A.E.K. Risø-M-1961, ISBN 87-550-0499-7, Metallurgy Department, Ris $\varnothing$ National Laboratory, DK-4000 Roskilde, Denmark, September 1977. 
[135] SchwalBe, K.-H.: Methoden zur Untersuchung der Rißausbreitung. In: Werkstoffermüdung und Bauteilfestigkeit: Prüfmethoden, Auswertung und Interpretation, Vorträge des DVM-Kolloquiums, 25./26. Sepr. 1980, TU Berlin, Seiten 105-118. Deutscher Verband für Materialforschung und -prüfung e.V., Berlin, 1981.

[136] Dally, J. W. and R. J. SAnford: Strain-Gage Methods for Measuring the Opening-Mode Stress-Intensity Factor, $K_{I}$. Experimental Mechanics, 27(4):381-388, 1987.

[137] Dally, J. W. and R. J. SAnford: On Measuring the Instantaneous Stress Intensity Factor for Propagating Cracks. Advances in Fracture Research, 5:3223$3230,1989$.

[138] Bürgel, A.: Bruchmechanische Kennwerte beim Wechsel im Versagensverhalten dynamisch scherbeanspruchter Risse. Doktorarbeit, Ruhr-Universität Bochum, Fakultät für Maschinenbau, Bochum, August 2000.

[139] Kalthoff, J. F.: Persönliche Mitteilung. Bochum, 2002.

[140] Berger, J. R. and J. W. Dally: An Overdeterministic Approach for Measuring $K_{I}$ using Strain Gages. Experimental Mechanics, 28(2):142-144, 1988.

[141] Bürgel, A., H. S. Shin, D. Bergmannshoff and J. F. Kalthoff: Optimization of the Strain-Gauge-Method for Measuring Mode-II Stress Intensity Factors. Proceedings of the $\mathrm{VII}^{\text {th }}$ Bilateral Czech/German Symposium: Significance of Hybrid Method for Assessment of Reliability and Durability in Engineering Sciences, April 13.-15. 1999, The Liblice Castle, pages 11-12, 1999.

[142] Bluhm, J. I.: Fracture Arrest. In Liebowitz, H. (editor): Fracture Design of Structures, volume V of Fracture, An Advanced Treatise, chapter 1., pages 2-60. Academic Press, New York, San Francisco, London, 1969.

[143] Barker, L. M.: Specimen Size Effects in Short-Rod Fracture Toughness Measurements. In Underwood, J. H., S. W. Freiman and F. I. Baratta (editors): Chevron-notched specimens, testing and stress analysis : a symposium sponsored by ASTM Committee E-24 on Fracture Testing, Louisville, Ky., 21 April 1983, volume ASTM STP 855, pages 117-133, Philadelphia, PA, 1984. American Society for Testing and Materials.

[144] Newman, J. R.: A Review of Chevron-Notched Fracture Specimens. In UnDerwood, J. H., S. W. Freiman and F. I. BARATTA (editors): ChevronNotched Specimens: Testing and Stress Analysis: a symposium sponsored by ASTM Committee E-24 on Fracture Testing, Louisville, Ky., 21 April 1983, 
volume ASTM STP 855, pages 5-31, Philadelphia, PA, 1984. American Society for Testing and Materials.

[145] Kobayashi, T.: Strength and Toughness of Materials. Springer-Verlag, Tokyo, 2004.

[146] Podleschny, R. and J. F. Kalthoff: A Novel Mode-II Fracture Criterion. In Proc. 10th European Conference on Fracture, Structural Integrity: Experiments, Models and Applications, ECF 10, 20.-23. Sept. 1994, Berlin, Germany, pages 211-221. Engineering Materials Advisory Services Ltd., West Midlands, UK, 1994.

[147] Kalthoff, J. F.: Transition in the Failure Behavior of Dynamically Shear Loaded Cracks. In Chen, D. F. (editor): Proc. 11th US National Congress of Applied Mechanics, Tuscon, Arizona, May 21-25, 1990, pages 247-250. also Appl. Mech. Rev., Vol. 43, Part 2, May 1990.

[148] Kalthoff, J. F.: Modes of Dynamic Shear Failure in Solids. In Keynote Paper, 13th US National Congress of Applied Mechanics Session Dynamic Failure Modes in Solids, University of Florida, Gainesville, Florida, June 21-26, 1998, pages 1-31. Special Issue of Int. Journ. of Fracture, Vol. 100, 2000.

[149] Kalthoff, J. F.: Schattenoptische Kaustikenmethode. In Rohrbach, Chr. (editor): Handbuch der experimentellen Spannungsanalyse, chapter D 2.9, pages 397-418. VDI-Verlag, Düsseldorf, 1989.

[150] Kanel, G. I., S. V. Razorenov and V. E. Fortov: Shock-Wave Phenomena and the Properties of Condensed Matter. Springer-Verlag, New York, 2004.

[151] Curran, D. R.: Failure Criteria in LECEI-Tests. unpublished laboratory report, December 2003.

[152] ASTM E 23-97: Standard Test Methods for Notched Bar Impact testing of Metallic Materials. Annual Book of ASTM Standards 03.01 Metals - Mechanical Testing, American Society for Testing and Materials, West Conshohocken, 1997.

[153] Kalthoff, J. F., W. Klemm and S. Winkler: Auswerteprogramm für instrumentierten Kerbschlagbiegeversuch. Fraunhofer-Institut für Werkstoffmechanik, Nr. V45/85, Freiburg, August 1985.

[154] Kerkhof, F.: Morphologie der Bruchfläche. In: Einführungskurs in die Bruchmechanik, Seiten F1-F22. Ernst-Mach-Institut der Fraunhofer Gesellschaft, Freiburg i. Br., 1969. 
[155] Kerkhof, F.: Morphologie der natürlichen"Bruchfläche. In: Bruchvorgänge in Gläsern, Kapitel 7, Seiten 209-220. Verlag der Deutschen Glastechn. Ges., Frankfurt a.M., 1970.

[156] Kerkhof, F.: Bruchmechanische Analyse von Schadensfällen. Glastechnische Berichte, 48(6):112-124, 1975.

[157] Angenheister, G. (editor): Landolt-Börnstein: Numerical Data and Functional Relationships in Science and Technology, New Series, Group 5, Vol. $1 b$ - Physical Properties of Rocks. Springer, Berlin, 1982.

[158] Grady, D. E. and M. E. KIPP: The Micromechanics of Impact Fracture of Rock. Int. J. Rock Mech. Min. Sci. \& Geomech. Abstr., 16:293-302, 1979.

[159] Shockey, D. A., C. F. Petersen, D. R. Curran and J. T. Rosenberg: Failure of Rock under High Rate Tensile Loads. In HARDY, H. R. JR. and R. Stefanko (editors): New Horizons in Rock Mechanics, Fourteenth Symp. On Rock Mechanics, University Park, Pennsylvania, June 11-14, 1972, pages 709-738. American Society of Civil Engineers, 1973.

[160] Rummel, F.: Fracture and Flow of Rocks and Minerals. In Angenheister, G. (editor): Springer Verlag, pages 141-238. Landolt-Börnstein: Numerical Data and Functional Relationships in Science and Technology, New Series, Group 5, Vol. 1b - Physical Properties of Rocks, Berlin, 1982.

[161] Atkinson, B. K.: Stress Corrosion and the Rate-Dependent Tensile Failure of a Fine-Grained Quartz Rock. Tectonophysics, 65(3-4):281-290, June 1980.

[162] Atkinson, B. K.: Subcritical Crack Growth in Geological Materials. J. Geophys. Res., 89(B6):4077-4114, June 1984.

[163] Atkinson, B. K. and P. G. Meredith: Experimental Fracture Mechanics Data for Rocks and Minerals. In Atkinson, B. K. (editor): Fracture Mechanics of Rock, chapter 11, pages 429-476. Academic Press, London, 1987.

[164] Baud, P., A. Schubnel and T.-F. Wong: Dilatancy, compaction, and failure mode in Solnhofen limestone. J. Geophys. Res., 105(B8):19289-19303, August 2000 .

[165] Renner, J. and F. Rummel: The effect of experimental and microstructural parameters on the transition from brittle failure to cataclastic flow of carbonate rocks. Tectonophysics, 258(1-4):151-169, June 1996.

[166] Holbrook, D. F. and C. G. Stone: Arkansas Novaculite - A Silica Resource. Williams, N. F. (editor), State of Arkansas - Arkansas Geological Commission, 
Little Rock, Arkansas, reprinted with minor revisions from: Thirteenth Annual Forum on the Geology of Industrial Minerals, 1978, Oklahoma Geological Survey Circular 79, 1979.

[167] Zietlow, W. K. and J. F. Labuz: Measurement of the Intrinsic Process Zone in Rock Using Acoustic Emission. Int. J. Rock Mech. \& Min. Sci., 35(3):291-299, 1998.

[168] Rummel, F. and C. Fairhurst: Determination of the Post-Failure Behaviour of Brittle Rock Using a Servo-Controlled Testing Machine. Rock Mechanics, 2(4):189-204, 1970.

[169] Hudson, J. A., E. T. Brown and C. FAirhurst: Optimizing the control of rock failure in servo-controlled laboratory tests. Rock Mechanics, 3(4):189-204, 1971.

[170] Labuz, J. F. and L. Biolzi: Experiments with Rock: Remarks on Strength and Stability Issues. Int. J. Rock Mech. \& Min. Sci., 44(4):525-537, 2007.

[171] Broberg, K. B.: Significance of Morphology Changes at a Propagating Crack Edge. International Journal of Fracture, 130(4):723-742, 2004.

[172] Gao, H.: Surface Roughening and Branching Instabilities in Dynamic Fracture. J. Mech. Phys. Solids, 41(3):457-486, 1993.

[173] Marder, M. and J. Fineberg: How Things Break. Physics Today, 49(9):2429, 1996.

[174] SchwalBe, K.-H.: Bruchmechanik metalllischer Werkstoffe. Carl Hanser Verlag, München, Wien, 1980.

[175] Liu, C., W.G. Knauss and A.J. Rosakis: Loading rates and dynamic initiation toughness in brittle solids. Int. Journ. of Fracture, 90(1-2):103-118, 1998.

[176] Alber, M. and E. Hauptfleisch: Generation and Visualization of Microfractures in Carrara Marble for Estimating Fracture Toughness, Fracture Shear and Fracture Normal Stiffness. Int. J. Rock Mech. \& Min. Sci., 36(8):1065-1071, December 1999.

[177] Nasseri, M. H. B., B. Mohanty and R. P. Young: Fracture Toughness Measurements and Acoustic Emission Activity in Brittle Rocks. Pure appl. geophys., 163(5-6):917-945, Juni 2006.

[178] Telenga, K. A., A. Bertram, J.F. Kalthoff and B. Stöckhert: Destiny in Tensile Crack Formation? Geology, submitted, 2008. 
[179] Atkinson, B. K.: Fracture Mechanics of Rock. Academic Press, London, 1987.

[180] Curran, D. R.: Shear Band and Tensile Crack Initiation Criteria in the LECEI-Test. unpublished laboratory report, December 2003.

[181] Broberg, K. B.: Differences Between Mode I and Mode II Crack Propagation. Pageoph, 163(9):1867-1879, September 2006. DOI 10.1007/s00024-006-0101-7.

[182] Broberg, K. B.: On Crack Paths. In Knauss, W. G., K. Ravi-Chandar and A. J. Rosakis (editors): Workshop on Dynamic Fracture, California Institute of Technology, Pasadena, February 17-18, 1983, pages 140-155, 1983.

[183] Batra, R.C. and M.V.S. Ravinsankar: Three-dimensional numerical simulation of the Kalthoff experiment. Int. Journ. of Fracture, 105(2):161-186, 2000 .

[184] Backers, T., E. Rybacki, M. Alber and O. Stephansson: Fractography of rock from the new Punch-Through Shear Test. In Dyskin, A. V., X. Hu and E. SAhouryeH (editors): Structural Integrity and Fracture: Proceedings of the International Conference, SIF 2002, Perth, Australia, 25-28 September 2002, pages 303-308, Lisse, 2002. A.A. Balkema.

[185] Curran, D. R.: Personal Communication. Bochum, March 2008.

[186] Curran, D. R.: Comparison of Mesomechanical and Continuum Granular Flow Models for Ceramics. In Furnish, M. D., M. Elert, T. P. Russell and C. T. White (editors): Shock Compression of Condensed Matter: AIP Conference Proceedings, Baltimore, Maryland (USA), 31 July-5 August, 2005, volume 845, pages 315-318. American Physical Society, December 2006.

[187] Peck, L., C. C. Barton and R. B. Gordon: Microstructure and the Resistance of Rock to Tensile Fracture. J. Geophys. Res., 90(B13):11533-11546, November 1985.

[188] Hashida, T. and H. Takahashi: Fracture Toughness Testing of Corebased Specimens by Acoustic Emission. Int. J. Rock Mech. \& Min. Sci., 30(1):6169, 1993.

[189] Bažant, Z. P., R. Gettu and M. T. Kazemi: Identification of Nonlinear Fracture Properties From Size Effect Tests and Structural Analysis Based on Geometry-dependent R-curves. Int. J. Rock Mech. Min. Sci. \& Geomech. Abstr., 28(1):43-51, 1991.

[190] Wawersik, W. R. and C. Fairhurst: A Study of Brittle Rock Fracture in Laboratory Compression Experiments. Int. J. Rock Mech. Min. Sci. \& Geomech. Abstr., 7(5):561-564, September 1970. 
[191] Dally, J. W., W. L. Fourney and G. R. Irwin: On the Uniqueness of the Stress Intensity Factor - Crack Velocity Relationship. International Journal of Fracture, 27(3-4):159-168, March 1987.

[192] Poliakov, A. N. B., R. Dmowska and J. R. Rice: Dynamic shear rupture interactions with fault bends and off-axis secondary faulting. J. Geophys. Res., 107(B11), November 2002. 2295, doi:10.1029/2001JB000572.

[193] Sagy, A., Z. Reches and I. Roman: Dynamic fracturing: field and experimental observations. J. Stuctural Geology, 23(8):1223-1239, 2001.

[194] Segall, P.: Rate-Dependent Extensional Deformation Resulting From Crack Growth in Rock. J. Geophys. Res., 89(B6):4185-4195, 1984.

[195] Grady, D. E. and J. Lipkin: Criteria for Impulsive Rock Fracture. Geophysical Research Letters, 7(4):255-258, 1980.

[196] Lankford, J.: Technical Note: The Role of Tensile Microfracture in the Strain Rate Dependence of Compressive Strength of Fine-Grained Limestone - Analogy with Strong Ceramics. Int. J. Rock Mech. Min. Sci. \& Geomech. Abstr., 18(2):173-175, 1981.

[197] Swanson, P. L.: Subcritical Crack Growth and Other Time-and EnvironmentDependent Behavior in Crustal Rocks. J. Geophys. Res., 89(B6):4137-4152, June 1984.

[198] Sato, K. and T. Hashida: Cohesive Crack Analysis of Toughness Increase Due to Confining Pressure. Pageoph, 163(5-6):1059-1072, 2006.

[199] Ashby, M. F. and C. G. Sammis: The Damage Mechanics of Brittle Solids in Compression. Pageoph, 133(3):489-521, 1990.

[200] Savage, J. C., J. D. Byerlee and D. A. Lockner: Is internal friction friction? Geophysical Research Letters, 23(5):487-490, March 1996.

[201] Costamagna, R., J. Renner and O. T. Bruhns: Relationship between fracture and friction for brittle rocks. Mechanics of Materials, 39(4):291-301, April 2007. doi:10.1016/j.mechmat.2006.06.001.

[202] Cocco, M., P. Spudich and E. Tinti: On the mechanical work absorbed on faults during earthquake ruptures. In Abercrombie, R., A. MCGARr, H. Kanamori and G. Di Toro (editors): Radiated Energy and the Physics of Earthquakes Faulting, volume 170 of Geophysical Monograph, pages 237-254. AGU, November 2006.

[203] Wawersik, W. R.: Detailed Analysis of Rock Failure in Laboratory Compression Tests. Ph. d. thesis, University of Minnesota, 1968. 
[204] Engvik, A. K., A. Bertram, J. F. Kalthoff, B. Stöckhert, H. AusTRHeim and S. Elvevold: Magma-driven Hydraulic Fracturing and Infiltration of Fluids into the Damaged Host Rock, an Example from Dronning Maud Land, Antarctica. J. Stuctural Geology, 27(5):839-854, 2005.

[205] Rubin, A. M.: Propagation of magma filled cracks. Annu. Rev. Earth Planet. Sci., 8:287-336, 1980.

[206] Segall, P.: Formation and Growth of Extensional Fracture Sets. J. Geophys. Res., 95(4):454-462, April 1984.

[207] Dally, J. W., W. F. Riley and J. S. Sirkis: Strain Gages. In Kobayashi, A. S. (editor): Handbook on Experimental Mechanics, chapter 3, pages 39-77. VCH Publishers, Inc., New York, second edition, 1993.

[208] McConnell, K. G. and W. F. Riley: Strain-Gage Instrumentation and Data Analysis. In Kobayashi, A. S. (editor): Handbook on Experimental Mechanics, chapter 4, pages 79-117. VCH Publishers, Inc., New York, second edition, 1993.

[209] Kalthoff, J. F.: Vorlesung Experimentelle Mechanik I - Grundlagen der Dehnungsmeßtechnik. Ruhr-Universität Bochum, SS 2000.

[210] KeIL, S.: Beanspruchungsermittlung mit Dehnungsmeßstreifen. Cuneus, Zwingenberg, 1995.

[211] RILEM Draft Recommendations: Size-effect method for determining fracture energy and process zone size of concrete. Materials and Structures, 23(6):461465, November 1990.

[212] Abaqus: Abaqus Online Documentation - Version 6.\%. Dassault Systèmes, 2007.

[213] I-DEAS Master Series ${ }^{\mathrm{TM}}$ : Exploring I-DEA $S^{T M}$ Simulation. Handbook, Volume I,II, 1996.

[214] Shin, C. F. and R. J. Asaro: Elastic-Plastic Analysis of Cracks on Bimaterial Interfaces: Part I - Small Scale Yielding. J. Appl. Mech., 55:299-316, 1988. 


\section{Anhang}

\section{A Ergänzende Messungen zur Bestimmung des Spannungs- feldes und des Bruchverhaltens von Gestein}

\section{Alternative Methoden zur Bestimmung der Rißspitzenspannungskonzentrationen}

Zur Überprüfung der Eignung des schattenoptischen Kaustikenverfahrens für Spannungsintensitätsfaktorbestimmungen an Gestein wurde zunächst eine schattenoptische Meßeinrichtung in Reflexion in der Spezifikation der vorhandenen, in Verbindung mit der Hochgeschwindigkeitskamera betriebenen Anlage erstellt. Sorgfalt wurde auf einen hochpräzisen, empfindlichen Aufbau gelegt, da die durch das Prinzip des Meßverfahrens gegebene Abhängigkeit der Größe der Kaustik von dem reziproken Wert des E-Moduls wegen der Höhe dieses Wertes für Gestein sehr große Effekte nicht erwarten ließ. Abbildung 56 zeigt eine Kaustik, die an einer Kalksteinprobe (Typ I, Rißlänge $13 \mathrm{~mm}$ ) bei einer Belastung von $K_{I}=0,5 \mathrm{MN} / \mathrm{m}^{3 / 2}$ aufgenommen wurde. Die Kaustik ist zwar deutlich sichtbar, aber wegen der geringen Größe und aufgrund der fehlenden scharfen Abgrenzung zum Schattengebiet zur quantitativen Kennwertbestimmungen wenig geeignet (siehe zum Vergleich die in Abb. 57 wiedergegebenen, an Polymetacrylat (PMMA) und einem hochfesten Stahl photographierte Kausti-
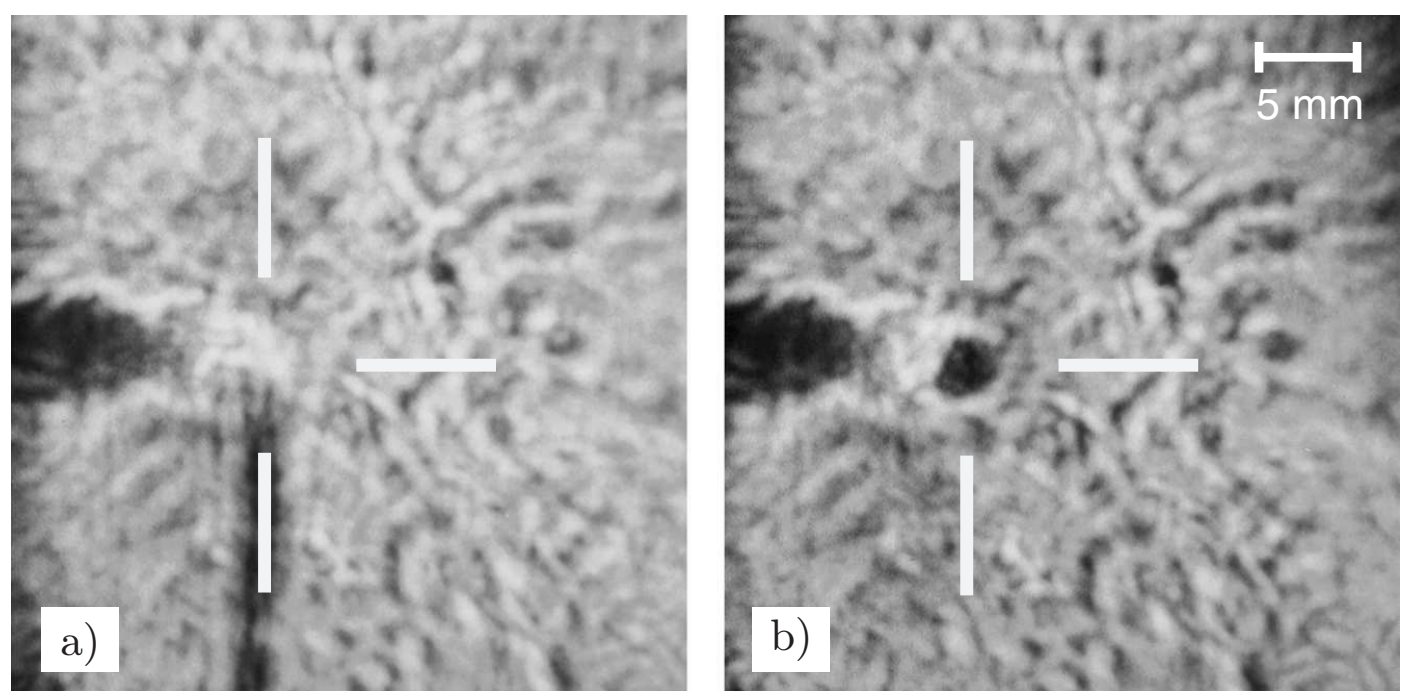

Abb. 56: Anwendung des schattenoptischen Kaustikenverfahrens auf Solnhofener Kalkstein: a) unbelasteter Zustand und b) Ausbildung einer Kaustik mit einem Schattenfleck von geringem Durchmesser sowie einer wenig ausgeprägten lichtstarken Grenzkurve. 

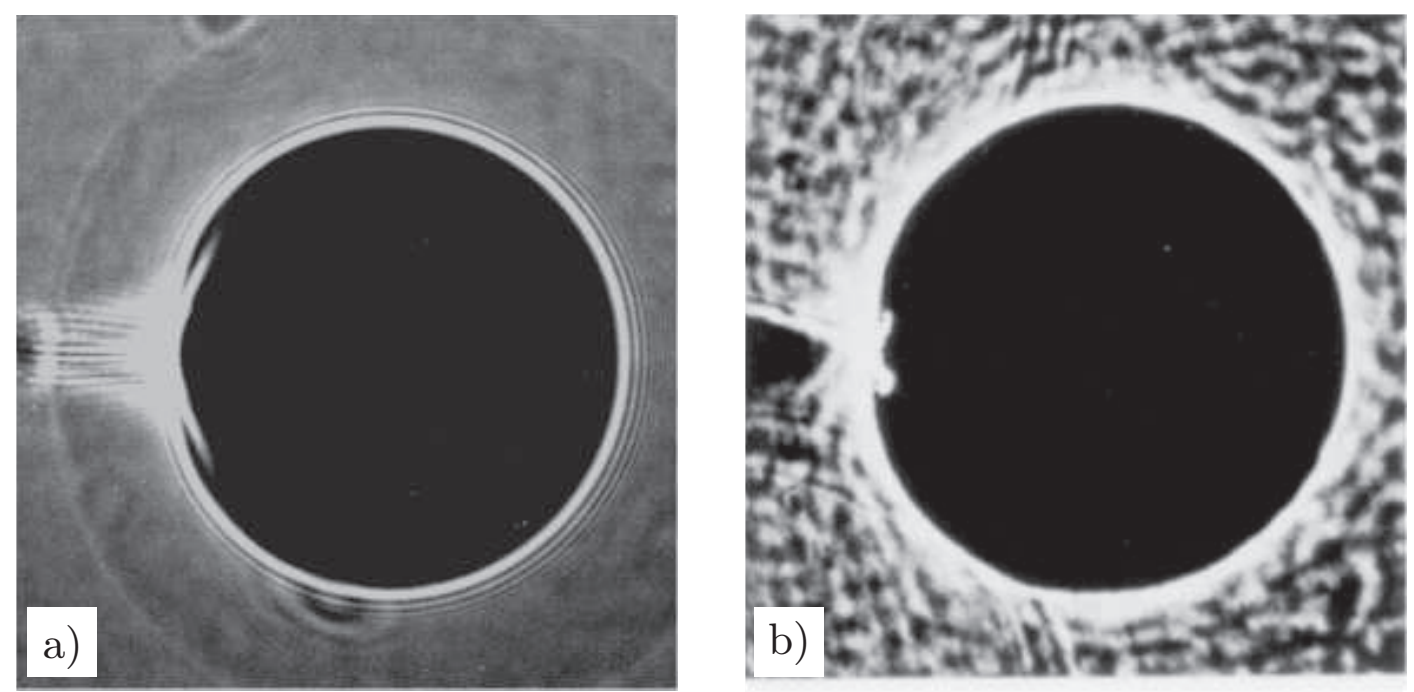

Abb. 57: Quantitativ verwertbare Kaustiken: a) PMMA (Transmission) und b) Stahl (Reflexion).

ken guter Qualität). Methodische Verbesserungen, so z. B. durch Veränderungen des Strahlengangs oder Anwendungen des Prinzips der Kaustikenvervielfachung (Vergrö§erung des schattenoptischen Effekts durch Mehrfachreflexionen) sowie durch Verwendung eines Oberflächenschicht-Kaustikenverfahrens (Übertragung der Vorgänge in Gestein auf eine an der Oberfläche aufgebrachte Schicht hoher schattenoptischer Empfindlichkeit) wurden erprobt, führten aber nicht zu befriedigenden Ergebnissen. Insgesamt wurde als Ergebnis festgestellt, daß das schattenoptische Verfahren bei Gesteinen für quantitative Messungen in der Praxis doch zu insensitiv und stattdessen ein anderes Verfahren einzusetzen sei.

Weiterhin wurde mit dieser Methode eine Besonderheit bei der Ausbildung von Rißspitzenspannungskonzentrationen bei grobkörnigen Gesteinen festgestellt. Abbildung 58 zeigt eine Serie von schattenoptischen Aufnahmen eines Experimentes an einer Granitprobe mit einem mittleren Korndurchmesser von $2 \mathrm{~mm}$. Die Belastung wurde quasistatisch (konstante Durchbiegungsrate: $v_{0}=10^{-6} \mathrm{~m} / \mathrm{s}$ ) durchgeführt und jeweils zur Gewinnung der Bilder angehalten. Die Bruchzähigkeit $K_{I c}^{s *}$ des Materials beträgt $1,9 \mathrm{MN} / \mathrm{m}^{-3 / 2}$, als Startriß wurde eine Kerbe mit einem Radius von 0,125 mm verwendet. Deutlich ist zu erkennen, daß sich neben dem Schattenfleck an der Rißspitze Spannungskonzentrationen an einzelnen Mikrorissen innerhalb der Prozeßzone ausbilden, selbst in Bereichen mit beträchtlicher Entfernung von der Rißspitze. Für ein solches Material sind zu einer kontinuumsmechanischen Betrachtung der Beanspruchung große Abstände zur Rißspitze einzuhalten. Es ist somit für die avisierten Untersuchungen ungeeignet.

Trotzdem bildet sich selbst in diesem Material global ein homogenes Spannungsfeld aus, wie ein Experiment mit dem spannungsoptischen Oberflächenschichtverfahren an demselben Granit zeigt (Abb.59). Die Oberflächenschicht gleicht die Effekte der 

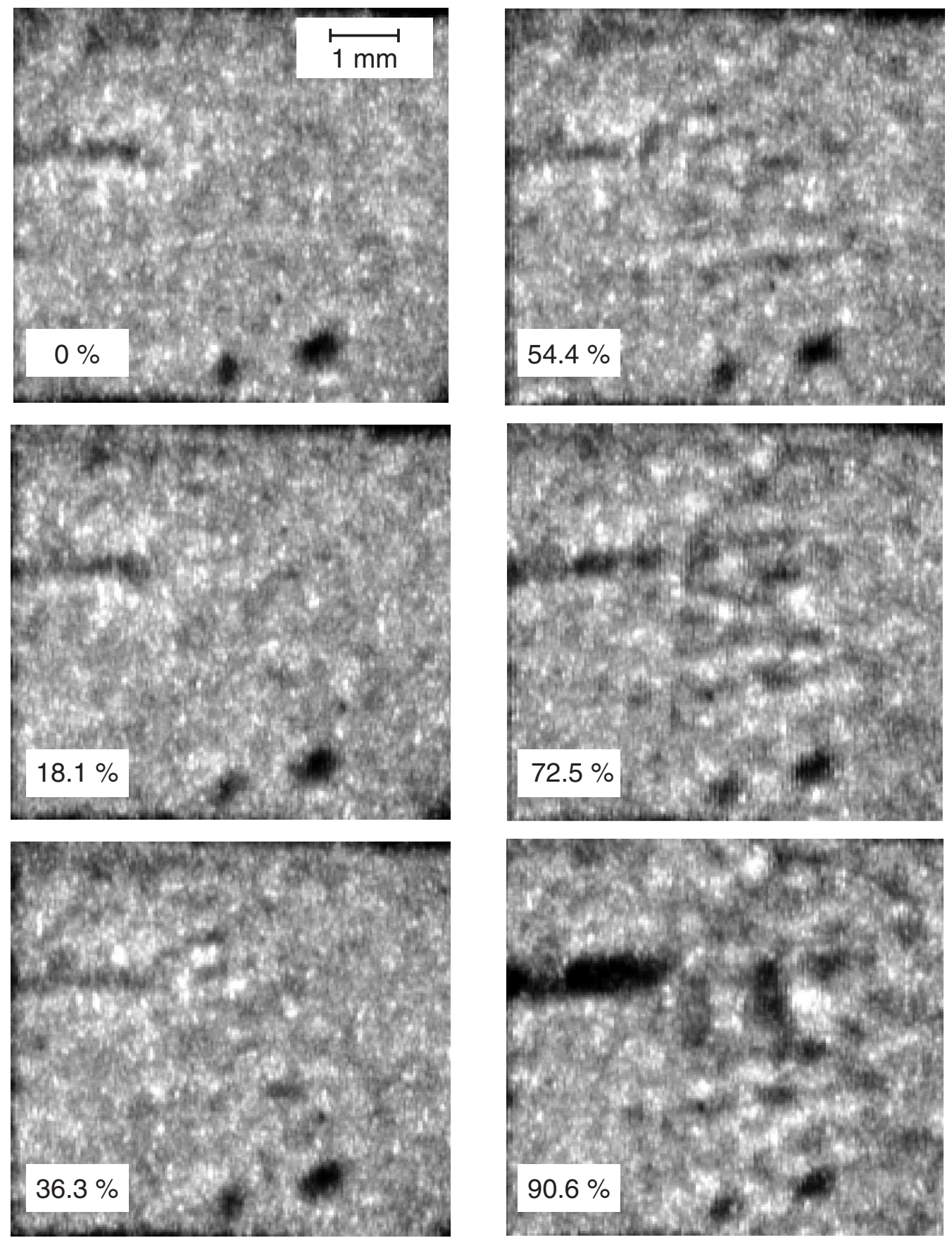

Abb. 58: Ausbildung von Spannungskonzentrationen an Prozeßzonenmikrorissen in Kerbspitzennähe während eines quasistatischen Experimentes an Granit (mittlerer Korndurchmesser: $2 \mathrm{~mm}$ ); Prozentzahlen beziehen sich auf $K_{I c}^{s}$.

Prozeßzone aus und es entstehen symmetrische Isochromaten (Linien gleicher Hauptspannungsdifferenz). Die anliegende Belastung beträgt $K_{I}=1,6 \mathrm{MN} / \mathrm{m}^{3 / 2}$. 


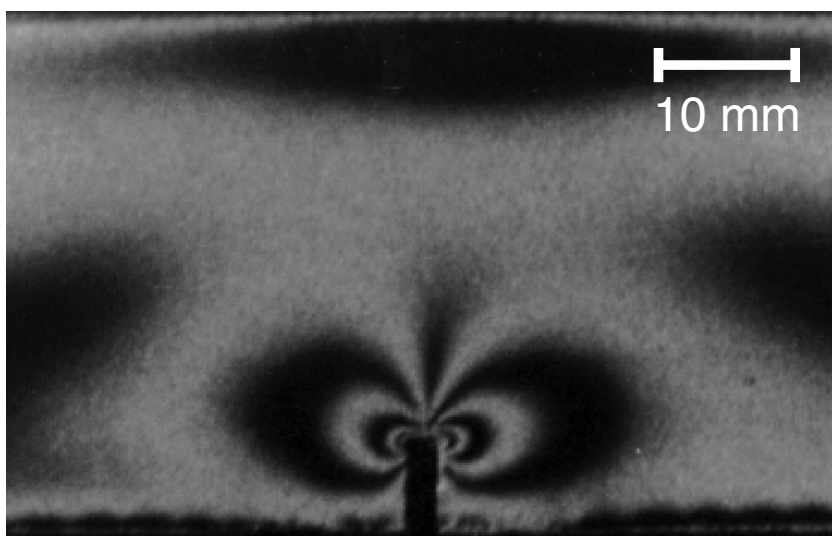

Abb. 59: Symmetrische Isochromaten der Oberflächenschicht in einem Experiment an Granit (mittlerer Korndurchmesser: $2 \mathrm{~mm}$ ).

Zusammenfassend läßt sich feststellen:

- Die Kaustikenmethode führte aufgrund der Materialbesonderheiten nicht zu quantitativ verwertbaren Resultaten, ermöglichte aber eine Klassifizierung der Materialien.

- Die Methode der Spannungsoptik bietet sich nicht an, da das untersuchte Material opaque ist und Rißausbreitungsvorgänge mit der Oberflächenschichtmethode nicht untersucht werden können. Experimente, die mit der Oberflächenschichtmethode durchgeführt wurden, zeigten jedoch, daß das vorliegende Spannungsfeld trotz der Mikrorißbildung homogen ist.

\section{Prozeßzonenentwicklung und Bruchausbreitungsverhalten in Marmor}

Ein mit dem Ziel der späteren mikroskopischen Analyse des Rißlaufereignisses (siehe Abb.49) durchgeführtes Experiment an Marmor zeigt, wie auch das oben angeführte Beispiel an Granit, daß sich in grobkörnigeren geologischen Materialien (die mittlere Korngröße liegt im Bereich von $30 \mu \mathrm{m}$ ) deutlich größere Prozeßzonen ausbilden als bei den im Rahmen der Hauptexperimente untersuchten Gesteinen. Die Initiierungszähigkeit des Materials beträgt $K_{I c}^{s *}=0,6 \mathrm{MN} / \mathrm{m}^{-3 / 2}$, als Initiierungsstelle wurde eine Kerbe mit einem Radius von $0,75 \mathrm{~mm}$ verwendet. Das Experiment wurde mit quasistatischer Belastung (konstante Durchbiegungsrate: $v_{0}=10^{-6} \mathrm{~m} / \mathrm{s}$ ) in einer Dreipunktbiege-Anordnung (SENB-Probe) durchgeführt. Abbildung 60 zeigt eine Serie von Bildern der Probenoberfläche, die im Verlauf des Experimentes mit herkömmlicher Phototechnik aufgenommen wurden. Für jedes Teilbild ist die Höhe der Belastung, bezogen auf die Initiierungszähigkeit, angegeben. Die Belastung wurde zur Aufnahme der Bilder angehalten, um diese eindeutig einer Belastungsstufe zuordnen zu können. Im Vergleich zu der niedrigsten Belastungsstufe bei $14 \%$ ist $\mathrm{zu}$ beobachten, daß sich bei einer Belastung von $57 \%$ vor der Kerbspitze eine 


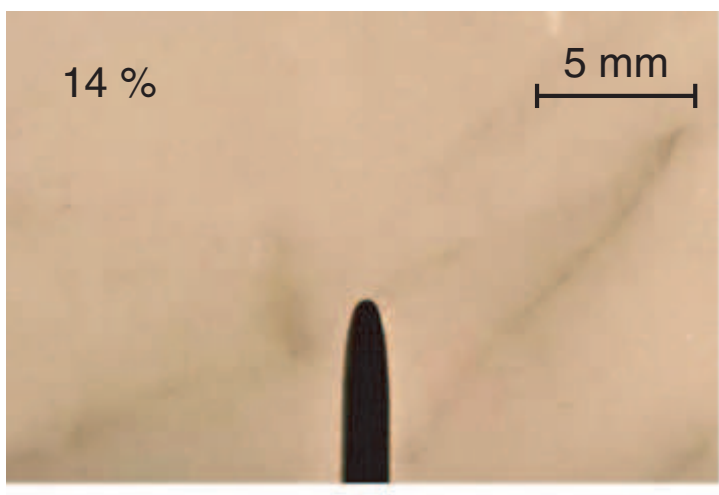

$57 \%$

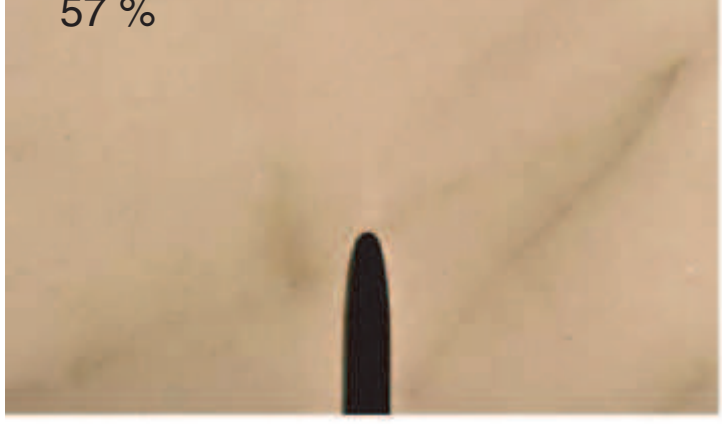

$62 \%$

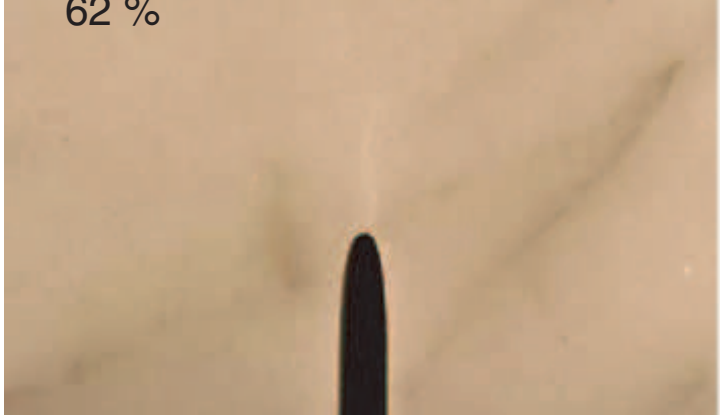

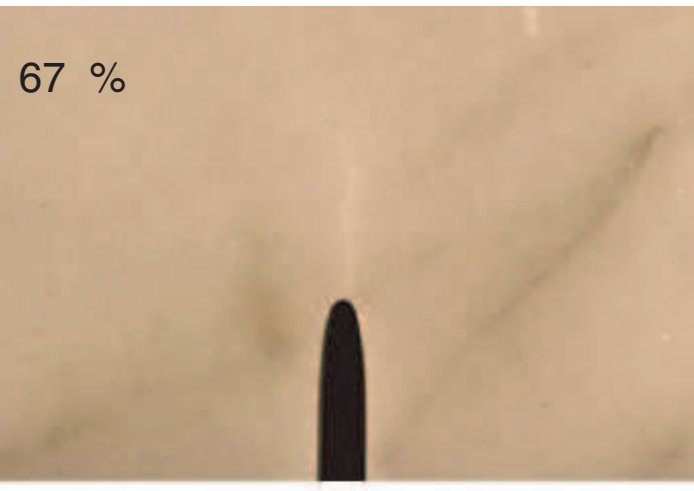

$74 \%$

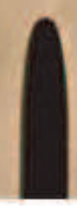

$76 \%$

Abb. 60: Quasistatisches Experiment an Marmor (siehe Mikroskopbild Abb. 49 in Kapitel 5); Prozentzahlen beziehen sich auf $K_{I c}^{s *}$.

Prozeßzone ausbildet. Diese ist als weißliches Gebiet endlicher Ausdehnung für das bloße Auge sichtbar. Im weiteren Verlauf breitet sich in Verlängerung der Starterkerbe ein makroskopischer Riß aus (bereits bei $67 \%$ der maximalen Belastung deutlich zu erkennen). Das Rißwachstum verläuft stabil und subkritisch, d.h. ohne weitere Lasterhöhung während der Belastungspausen erfolgt keine Rißerweiterung. Entsprechend diesem Bruchverhalten wächst der Rißwiderstand des Materials mit steigender Rißlänge, erkennbar daran, daß der Durchmesser der Schädigungszone entlang des Rißufers zunimmt. Erst nach dem Austritt des Risses aus dem Gesichtsfeld der Kamera tritt Instabilität ein und das Rißlaufexperiment wird unkontrollierbar. 


\section{B Zur Meßgenauigkeit/Mehrdeutigkeit von Dehnungsmeß- streifensignalen bei laufenden Rissen}

Um den Einfluß der Variation der Parameter, die den Kurvenverlauf von Dehnungsmeßstreifensignalen bei laufenden Rissen bestimmen und somit möglichen Signalverfälschungen unterliegen oder dazu führen können, zu veranschaulichen, wurden in Form von Modellkurven Meßsignale anhand von synthetischen Daten erzeugt. Die hier verglichenen Parameter sind:

- der Spannungsintensitätsfaktor $K_{I}$ bzw. die Rißlaufzähigkeit $K_{I D}$ und

- die Rißlaufgeschwindigkeit $v_{c}$ als avisierte Meßergebnisse, sowie andererseits

- die Konstante $A_{1}$ des zweiten Störterms (wie beschrieben, wird der erste Störterm, der $B_{0}$-Term bei optimierter Anordnung immer eliminiert).

Modellkurven für Meßketten bestehend aus jeweils fünf Dehnungsmeßstreifen/Meßstellen mit regelmäßigem, für alle Berechnungen gleichem Abstand und ansonsten den Bedingungen der experimentellen Meßwertermittlung nachempfundenen Vorgaben werden im folgenden gegenübergestellt.

\section{Rißbeschleunigung}

Als Referenz dienen die in Abb.61a dargestellten Signale. Für diese ist die Rißausbreitungsgeschwindigkeit $\left(v_{c}>0\right.$ für $t>0$ und $\left.d v_{c} / d t=0\right)$ sowie die Rißlaufzähigkeit konstant $\left(K_{I D}^{0}=1\right)$, desweiteren liegt kein Störtermeinfluß vor $\left(A_{n}, B_{m}=0\right.$, mit $n=1,2,3 \ldots$ und $m=0,1,2,3 \ldots)$. Die Einheiten dieser Größen, sowie der Längen- und
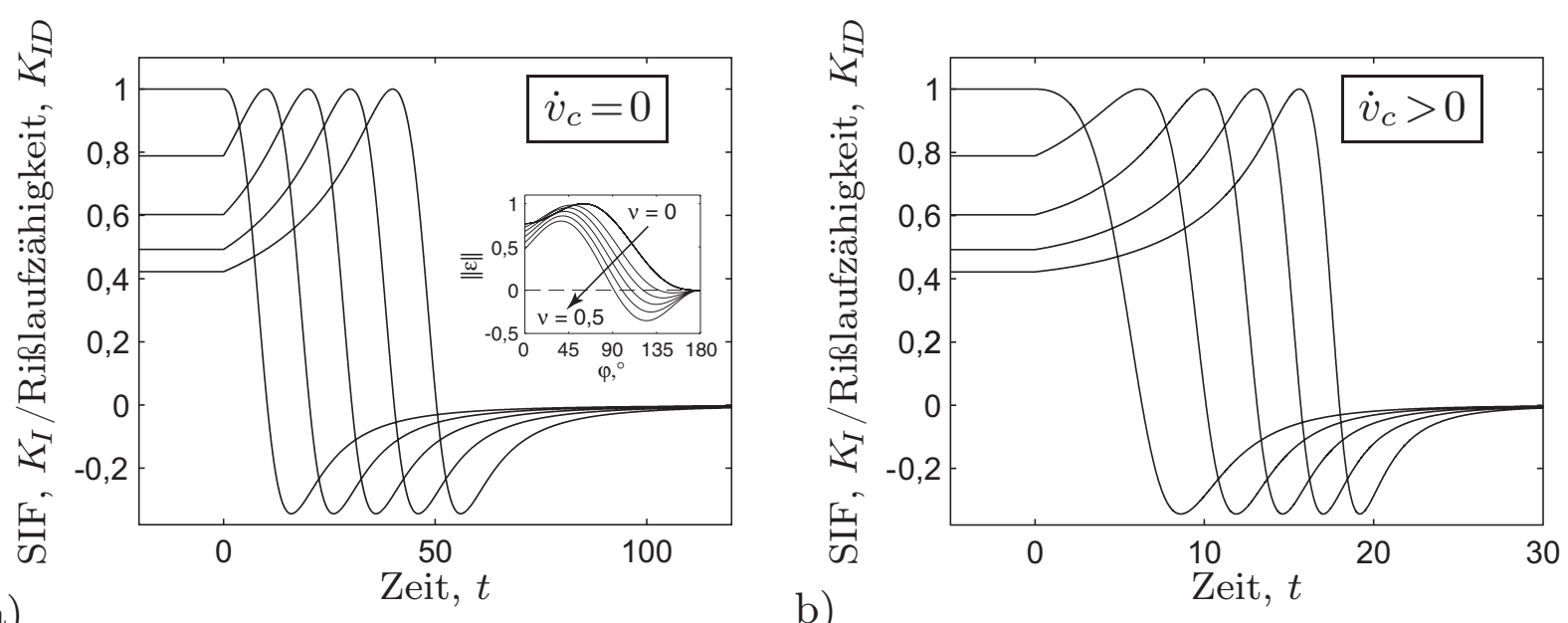

b)

Abb. 61: Synthetisch erzeugte Dehnungsmeßstreifen-Meßsignale: Spannungsintensitätsfaktoren/Rißlaufzähigkeiten a) eines sich mit konstanter Geschwindigkeit und b) eines sich beschleunigt ausbreitenden Risses. Die Kurvenform besonders deutlich zu erkennen beim Vergleich der der Minima der Signale - wird zusätzlich durch die Poissonzahl des Materials beeinflußt. 
Zeiteinheit sind willkürlich, werden jedoch, wie auch die Anfangswinkelpositionen, für sämtliche der nachfolgend gezeigten Beispiele konstant gehalten, so daß die relativen Bezüge skaliert sind. Auch die Poissonzahl $\nu$ wurde für jede der folgenden Berechnungen einheitlich gewählt: die Auswirkung einer Variation der Poissonzahl auf die Kurvenform ist als ,inset" in Abb.61a für die normierte Dehnung als Funktion des Winkels $\varphi$ angegeben.

Für den ersten Dehnungsmeßstreifen der Meßkette ist das Meßsignal bzw. der Spannungsintensitätsfaktor bei Beginn der Rißausbreitung (bei $t=0$ ) bereits maximal, d.h. gleich der Rißlaufzähigkeit, während die Signale der Dehnungsmeßstreifen, auf die die Rißspitze sich weiterhin zubewegt, ansteigen und nacheinander ebenfalls ihr Maximum erreichen. Sämtliche Signale haben denselben Abstand, die gleiche Signalform und -höhe, sie weisen alle dieselbe Rißlaufzähigkeit auf. Die Signale sind nach dem Durchschreiten des Maximums identisch. Dagegen ist für das in Abb. 61b angegebene Beispiel eines sich gleichförmig beschleunigt ausbreitenden Risses $\left(d v_{c} / d t=\right.$ const. $\left.>0\right)$ die Signalform der einzelnen Signale verschieden. Die steigende Ausbreitungsgeschwindigkeit bildet sich als „Stauchung“ der Signale auf der Zeitachse ab, die Meßsignale werden zunehmend steiler (die Rißlaufzähigkeit wurde konstant gehalten). Die Änderung der Kurvenform zeigt sich im Vergleich der Dehnungsmeßstreifen untereinander und mit den Referenzkurven der linken Seite der Abbildung. Aber auch für einen einzelnen Dehnungsmeßstreifen (für dessen Passieren die Rißspitze einen endlichen Zeitraum benötigt) ist im Verlauf der Ausbreitung eine Steigungszunahme infolge der Beschleunigung der Rißspitze erkennbar. Bereits hier wird deutlich (z. B. anhand der, trotz der kontinuierlichen Geschwindigkeitszunahme, im Vergleich zu der des ersten Dehnungsmeßstreifens relativ geringen Änderung der Kurvenform bei den beiden letzten Meßsignalen), daß eine Auswertung von Meßsignalen, die nicht in der hier angegebenen optimalen Form $\left(d v_{c} / d t=\right.$ const., kein Signalrauschen) nach Rißlaufzähigkeit und -geschwindigkeit nicht ohne Schwierigkeiten durchzuführen ist.

Die Abstände $r$ der Dehnungsmeßstreifen zur Rißspitze und die zugehörigen Meßwinkel $\varphi$ sind für die oben diskutierten Beispiele in Abb. 62 vergleichend nebeneinandergestellt. Auffallend ist, daß die Signale nach dem Durchschreiten des Minimums der Entfernung/Maximums der Spannungsintensität für das Referenzbeispiel mit konstanter Rißausbreitungsgeschwindigkeit identisch sind (Abb.62a). Sie unterscheiden sich hauptsächlich durch die, von der unterschiedlichen Anfangsentfernung verursachten Steigungsvariationen im Anfangsbereich der Kurven. Wohingegen für die beschleunigte Rißausbreitung (Abb. 62b) auch für den Bereich nach dem Extremwert Unterschiede festzustellen sind: hier ändert sich die Steigung durch die Geschwindigkeitszunahme, so daß sich die Kurven bei fortschreitender Zeit kontinuierlich annähern. Analog gilt dies ebenso für die Spannungsintensitätsfaktormodellkurven (Abb. 61), im Gegensatz zu der konstanten Geschwindigkeit in Abb. 61a variiert die Kurvenform, trotz der konstanten Rißlaufzähigkeit, für die beschleunigte Rißausbreitung (Abb.61b) im gesamten Kurvenverlauf. 

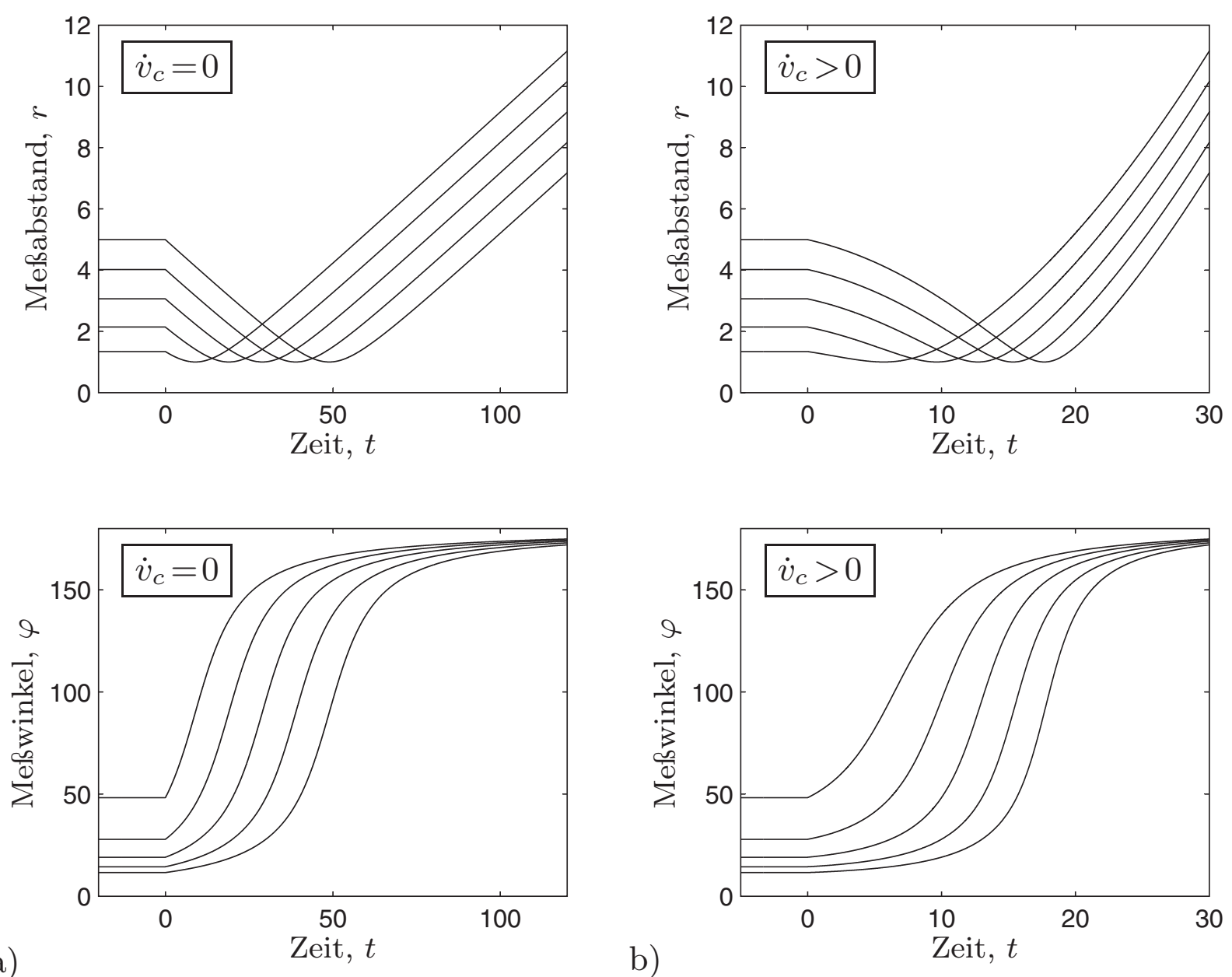

Abb. 62: Synthetisch erzeugte Dehnungsmeßstreifen-Modellkurven: Meßabstand (oben) und Meßwinkel (unten) eines sich a) mit konstanter Geschwindigkeit und b) beschleunigt ausbreitenden Risses.

\section{Änderung von $K_{I}$ und $A_{1}$}

Während für die vorstehenden Beispiele lediglich Kurvenformvariationen auf der Zeitachse auftraten, wird für die folgende Fallunterscheidung durch Änderung der Rißlaufzähigkeit (RZ, $K_{I D}$ ) und des $A_{1}$-Terms der Spannungs-/Dehnungsverteilung die Signalhöhe beeinflußt. Die Steigerung des Spannungsintensitätsfaktors beträgt hierbei $d K_{I} / d t=1 / 100$ (beginnend bei $t=0$ ) und für $A_{1}$ wurden in gleichmäßiger Abstufung Werte zwischen $-0,5 K_{I D}^{0}$ und $0,5 K_{I D}^{0}\left(K_{I D}^{0}=1\right.$, s. o. - die dritte Kurve mit $A_{1}=0$ ist somit identisch mit dem mittleren Signal des Referenzkurvesatzes) eingesetzt. Die entsprechenden Modellkurven finden sich in Abb.63a (Variation von $K_{I D}$ ) und Abb.63b (Variation von $A_{1}$ ), wobei bei letzterem die Variation nur für eine - die mittlere - Meßstelle durchgeführt wurde. Für beide Kurvensätze resultieren Änderungen sowohl für die Amplitude als auch für den zeitlichen Verlauf, erkennbar durch 


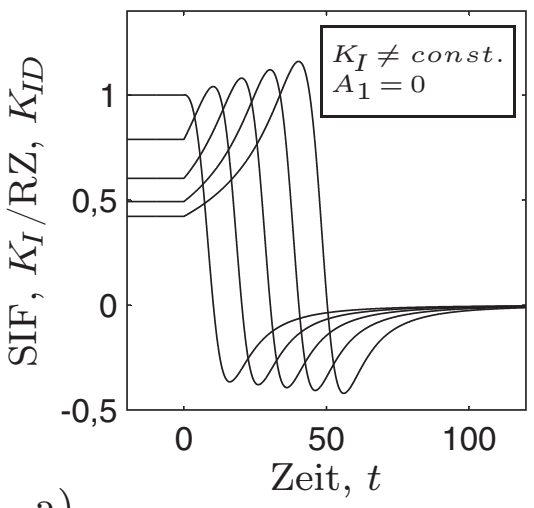

a)

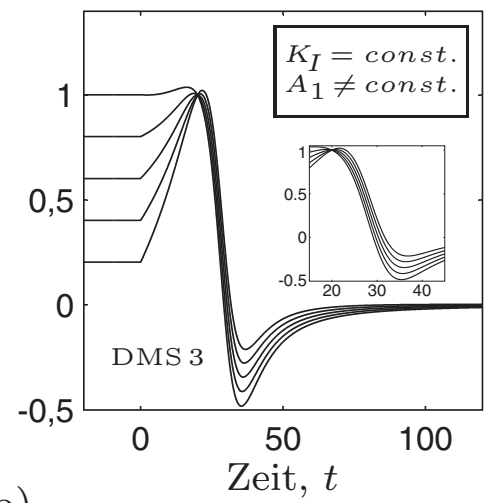

b)

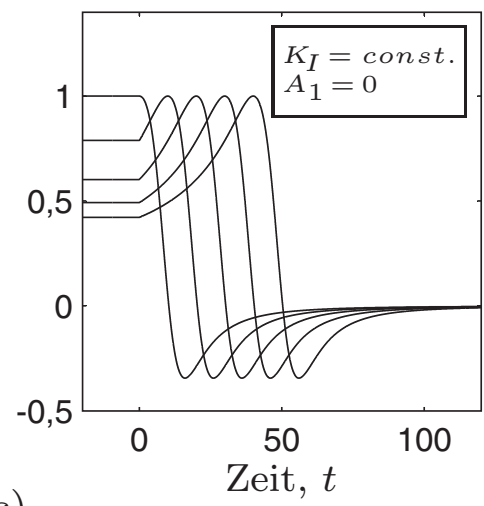

c) Abb. 63: Synthetisch erzeugte Dehnungsmeßstreifen-Modellkurven für: a) meh-
rere Meßstellen bei steigender $R$-Kurve und b) unterschiedliche $A_{1^{-}}$ Störtermwerte einer Meßstelle sowie: c) zum Vergleich die Modellkurven aus Abb. 61a.

die Abweichung vom parallelen Verlauf der Kurven, wie sie bei dem Referenzmodell vorliegen (zum Vergleich nochmals in Abb. 63c angegeben). Dabei ist die Änderung der Kurvenform in Amplitude und Zeit für beide Parametervariationen ähnlich, wie der in Abb. 64 in normierter Form zusammen mit den Referenzkurven aus (Abb.61a) dargestellte Vergleich zeigt. Für den Vergleich werden für die $A_{1}$-Variation wieder die Daten für eine Kette von Meßstellen herangezogen. Beginnend mit $A_{1}=0,5$ für die erste Meßstelle wurde in absteigender Reihenfolge jeweils einer der o. a. $A_{1}$-Werte angesetzt. Geschwindigkeitsänderungen machen sich durch Steigungsänderungen (i. e. Stauchung/Streckung auf der Zeitachse/in $x$-Richtung) bemerkbar, Änderungen von $K_{I} / K_{I D}$ nur in der Amplitude (die implizierte Steigungsänderung führt nicht zu Fehlinterpretationen, da die relative Position der Maxima und Minima unverändert bleibt). Zusätzliche Störtermeinflüsse betreffen beide Merkmale (und zudem die Position der Extremstellen) und somit könnte die Kurvenformänderung fälschlicherweise auch als Geschwindigkeitsänderung interpretiert werden. D.h. durch Vorliegen der Störtermeinflüsse ( $A_{1}$ wird bei Messungen mit Rißausbreitung „nur“ minimiert) ist das Gleichungssystem aus den Gln.(36) bzw. (37) und (38) unterbestimmt und es entsteht eine Mehrdeutigkeit. Gleichzeitig kann nicht angegeben werden, ob durch den Einfluß des $A_{1}$-Störterms, der sich der Rißlaufzähigkeit überlagert, eher zu geringe oder zu hohe Ergebnisse erzielt werden. Keine Verbesserung läßt sich erzielen, wenn zur Erhöhung des Bestimmtheitsgrades mehrere Meßsignale pro Meßstelle eingesetzt werden (etwa durch den Einsatz mehrerer Dehnungsmeßstreifen in unterschiedlicher Entfernung zur Rißspitze pro Meßstelle oder durch Auswertung benachbarter Dehnungsmeßstreifen). Da der Einfluß des $A_{1}$-Terms oder weiterer Störterme mit dem Abstand von der Rißspitze zunimmt und zudem im Verlauf des Rißfortschritts nicht notwendigerweise konstant ist, sind die Ergebnisse verschiedener Meßsignale nicht gemeinsam interpretierbar, das Gleichungsystem bleibt unterdeterminiert. 

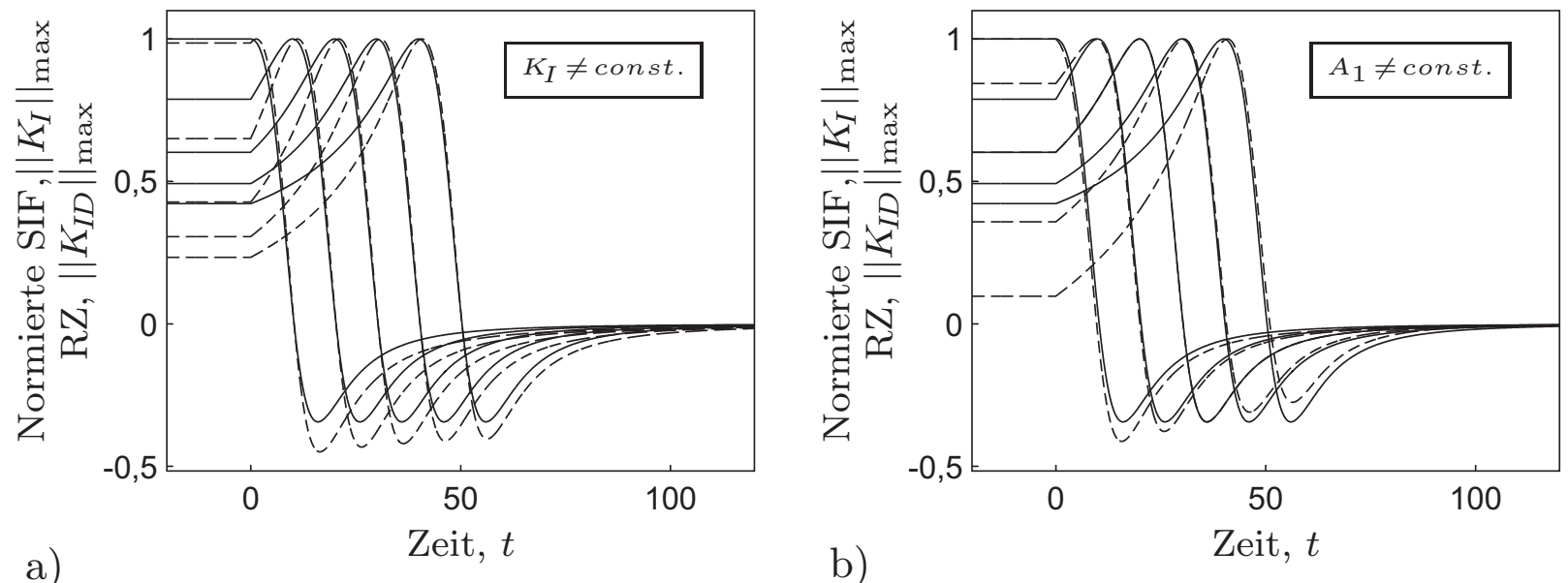

Abb. 64: Vergleich der, auf das Maximum des jeweiligen Signals normierten, Daten aus Abb. 63 (gestrichelte Linien) mit den Referenzkurven (durchgezogene Linien): a) mehrere Meßstellen bei steigender $R$-Kurve (Abb. 63a) und b) unterschiedliche $A_{1}$-Störtermwerte der Meßstellen (Abb.63b). 


\section{Bestimmung von Spannungsintensitätsfaktoren}

\section{Dehnungsmeßstreifen-Methode}

Spannungsintensitätsfaktoren werden bei Verwendung der DehnungsmeßstreifenMethode aus Dehnungsmeßstreifensignalen $\epsilon_{\text {DMS }}$ bestimmt (siehe Abs. 3.2). Dabei wird die mechanische Dehnung durch die Änderung des elektrischen Widerstandes bei der Längenänderung des Dehnungsmeßstreifens festgelegt [22,207-210]).

Bestimmt wird die Dehnung $\epsilon^{\alpha}$ in Meßrichtung $\alpha$ (siehe Abb. 21, Querdehnungen und Temperatureinflüsse sind vernachlässigbar) des Materials unter dem Dehnungsmeßstreifen. Die Empfindlichkeit des Dehnungsmeßstreifens ist dabei gegeben durch die Beziehung: $\Delta R_{s} / R_{s}=k_{s} \epsilon^{\alpha}$ zwischen der aufgenommenen Dehnung $k_{s} \epsilon^{\alpha}$ als Eingangsgröße und der verursachten Widerstandsänderung $\Delta R_{s} / R_{s}$ (der „ $k$-Faktor“ $k_{s}$ ist eine spezifische Proportionalitätskonstante). Bei der verwendeten WheatstoneViertelbrücken-Schaltung (Abb.65), bestehend aus einem aktiven Dehnungsmeßstreifen $\left(R_{s}\right)$ und drei Blindwiderständen $\left(R_{2,3,4}\right)$, bewirkt eine Widerstandsänderung $\Delta R_{s} / R_{s}$ als Meßsignal einen Spannungsabfall $\Delta U$ :

$$
\Delta U=\frac{R_{s} R_{2}}{\left(R_{s}+R_{2}\right)^{2}} \frac{\Delta R_{s}}{R_{s}} U_{0}=\frac{R_{s} R_{2}}{\left(R_{s}+R_{2}\right)^{2}} k_{s} \epsilon^{\alpha} U_{0}
$$

über der Brücke, die durch eine Spannung $U_{0}$ gespeist wird. Mit der maximalen Leistung $P_{s}^{\max }=R_{s} /\left(R_{s}+R_{2}\right)^{2} U_{0}$ ergibt sich die gemessene Dehnung $\epsilon_{\mathrm{DMS}}=\epsilon^{\alpha}$ zu:

$$
\epsilon_{\mathrm{DMS}}=\frac{R_{s}+R_{2}}{R_{2}} \frac{\Delta U}{\sqrt{R_{s} P_{s}^{\max }} k_{s}} .
$$

Aus dieser wurden unter Verwendung der nachfolgend tabellierten optimalen Applikationswinkel nach den Gln. (46) und (45) Modus-I- bzw. Modus-II-Spannungsintensitätsfaktoren bestimmt.

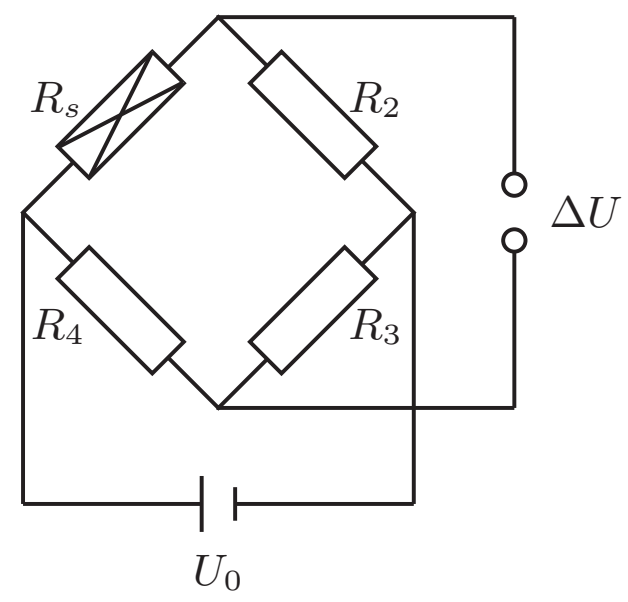

Abb. 65: Wheatstone-Viertelbrücken-Schaltung: $R_{s}$ - aktiver Dehnungsmeßstreifen, $R_{2,3,4}$ - Widerstände, $U_{0}$ - Speisespannung und $\Delta U$ - Meßsignal. 
Optimale Applikationswinkel, jeweils angepaßt an die Poissonzahl der untersuchten Gesteine:

\begin{tabular}{|l||c|c|c|c|}
\hline Gesteinstyp & Poissonzahl & $\alpha_{\mathrm{opt}}^{I, I I}$ & $\varphi_{\mathrm{opt}}^{I}$ & $\varphi_{\mathrm{opt}}^{I I}$ \\
\hline \hline Solnhofener Kalkstein & & & & \\
Arkansas Novaculit & 0,3 & 61,3 & 65,2 & 9,7 \\
\hline
\end{tabular}

Abschließend seien die experimentellen Details angeführt: Der $k$-Faktor der eingesetzten Dehnungsmeßstreifen ist 2,02, der Grund- oder Nennwiderstand beträgt $R_{s}=120 \Omega$ ebenso wie die Widerstände $R_{2,3,4}$. Als Speisespannung wurde $U_{0}=6 \mathrm{~V}$ angelegt und das Meßsignal zudem 100-fach verstärkt.

\section{Standard-Methode}

In Experimenten unter quasistatischer Belastung wurden Spannungsintensitätsfaktoren gleichzeitig nach der standardmäßigen Methode aus der anliegenden Last $F$ bestimmt. In den Drei-Punkt-Biege-Experimenten ergibt sich für die verwendeten Proben des Typs I bzw. Typs A (siehe Tabelle 3) der Spannungsintensitätsfaktor aus:

$$
K_{I}=\sigma \sqrt{\pi a} f(a / W) ;
$$

hierbei ist $W$ die Probenhöhe und $a$ die Rißlänge. Die Spannung ergibt sich nach: $\sigma=6 M / B W^{2}$ aus dem Biegemoment $M=F s / 4$, dabei bezeichnet $B$ die Probendicke und $s$ den Auflagerabstand. In den Experimenten wurde ein Verhältnis zwischen Auflagerabstand und Probenhöhe von 2,5 eingestellt. Für dieses Verhältnis lautet der geometrieabhängige Faktor $f(a / W)[211]$ :

$$
f(a / W)=\frac{1-2,5(a / W)+4,49(a / W)^{2}-3,98(a / W)^{3}+1,33(a / W)^{4}}{(1-a / W)^{3 / 2}}
$$




\section{Ablauf des LECEI-Experimentes}

Anhand eines Beispiels wird der vollständige Ablauf eines LECEI-Experimentes beschrieben. Dazu ist in Abb. 66 eine Serie von Bildern, die mit der Cranz-SchardinHochgeschwindigkeitskamera im Verlauf eines Experimentes an Solnhofener Kalkstein aufgenommen wurden, abgebildet. Zur Verwendung mit der Hochgeschwindigkeitskamera in einer Reflektionsanordnung ist die vordere Probenoberfläche an zwei Stellen verspiegelt. Als Spiegel wurden wenige Atomlagen messende Aluminiumschichten in einem speziellen Verfahren von einer zuvor bedampften Trägerplatte mittels einer dünnen Schicht eines Epoxid-Klebstoffes derart aufgebracht, daß der Rißausbreitungsvorgang durch die Spiegel nicht beeinflußt wird. Die Position der Spiegel, an der Lasteinleitungsstelle und unterhalb der Starterkerbe (siehe Abb. 66), gewährleisten sowohl die Kontrolle des Belastungsvorganges als auch des Rißlaufereignisses.

Die Photoserie (Abb. 66) zeigt, wie sich nach dem Aufschlag des Projektils eine Oberflächenwelle in der Probe ausbreitet. Da die Ausbreitungsgeschwindigkeit der Oberflächenwelle gering gegenüber den Körperwellen ist, erfolgt der Beginn Rißspitzenbeanspruchung bereits vor dem Eintreffen dieses Wellentyps an der Rißspitze. Der Beanspruchungsbeginn wird mit dem Rißspitzendehnungsmeßstreifen bestimmt, der zur Kontrolle der Initiierungsbedingungen ebenfalls auf der Probe appliziert ist. Dadurch wird der Zeitursprung $t_{0}$ festgelegt, auf den sich sämtliche Zeitangaben in den Teilbildern beziehen. Vom Zeitpunkt $t_{0}$ an, unter Berücksichtigung der späteren Rißausbreitung, wird die Rißspitzenbeanspruchung für ca. $30 \mu$ s nicht durch von den Probenrändern reflektierten Wellen beeinflußt. Nach einer sehr kurzen Phase der Beanspruchung durch die Wellenbelastung ist zu erkennen, daß von der scher(ModusII)-beanspruchten Rißspitze, im Winkel von etwa $70^{\circ}$, ein Zug(Modus-I)-Riß initiiert. Nach einem kurzen Rißlaufweg erreicht der Riß seine maximale Geschwindigkeit, angezeigt durch das Phänomen der Rißgabelung. Die Rißgabelung, die das Ende des Experimentes festlegt, erfolgt zu einem Zeitpunkt, bevor das Eintreffen sekundärer Wellen das Rißspitzenspannungsfeld stört.

Das Ausbilden von Rißspitzenkaustiken kann mit dem verwendeten optischen Aufbau nicht beobachtet werden, da die Kamera mit der Zielsetzung lediglich den zeitlichen Ablauf des Experimentes festzuhalten auf die Probenoberfläche fokussiert wurde. Die Kenntnis des zeitlichen Ablauf des Experimentes durch die gleichzeitige Beobachtung beider Spiegel ermöglicht es festzustellen, daß das Rißlaufereignis nicht durch Versagensvorgänge beeinflußt wird, die von der Lasteinleitungsstelle ausgehen. 

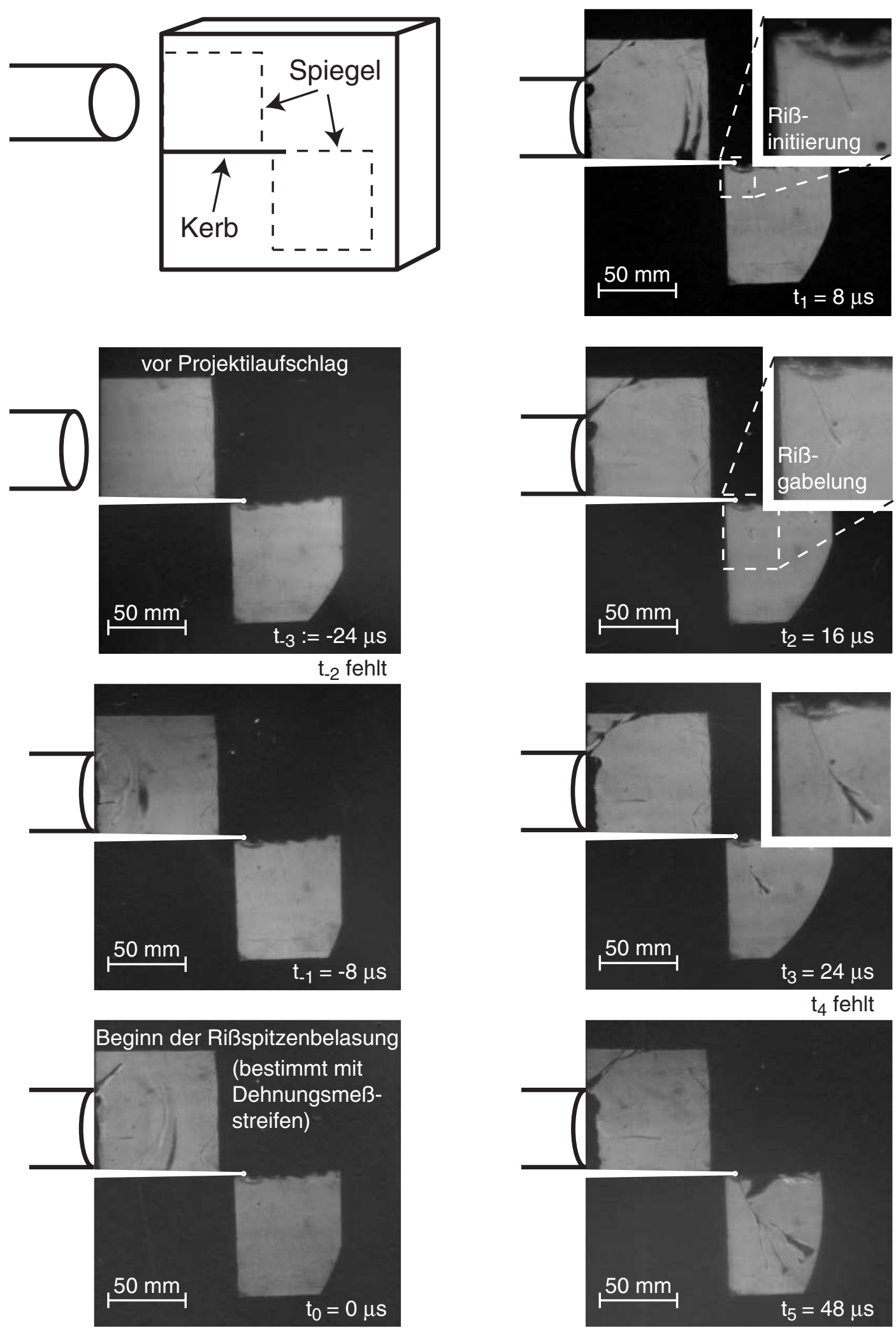

Abb. 66: Vollständiges LECEI-Experiment an Solnhofener Kalkstein. 


\section{E Finite-Elemente-Modellierung des LECEI-Experimentes}

Für die Experimente, die mit der LECEI-Belastungsmethode durchgeführt wurden, konnten keine Projektile aus demselben Material, aus dem die Proben bestanden, eingesetzt werden. Dies stellt eine Abweichung von der üblichen Verwendungsweise der Methode dar. Zur Beurteilung der Einflüsse des daraus resultierenden Impedanzkontrastes zwischen Probe und Projektil wurden mit der Finite-Elemente-Methode (FEM) dreidimensionale numerische Modellberechnungen des Experimentes durchgeführt. Zudem wurde der Einfluß der Stoßgeschwindigkeit auf den Aufbau der Rißspitzenspannungsintensitäten überprüft.

Die Berechnungen wurden mit dem Softwarepaket Abaqus [212] für linear-elastisches Materialverhalten durchgeführt. Es wurden zwei Finite-Elemente-Modelle generiert, die in Abb. 67 dargestellt sind. Die Modelle wurden bezüglich der Proben gemäß den Abmessungen der Laborexperimente erstellt (Tabelle 3, Typ II) und unterscheiden sich dadurch, daß eines der Modelle eine Kerbe und das andere einen scharfen Riß enthält. Vernetzung und Randbedingungen der Modelle, die mit dem Programm „IDeas" (Pre-Processing-Software, [213]) erstellt wurden, sind identisch. Lediglich im Nahbereich um die Kerbspitze wurden Anpassungen an die gegenüber dem scharfen Riß geänderte Geometrie vorgenommen. Das Netz ist aus quaderförmigen Elementen mit jeweils 8 Knoten aufgebaut. Die Gesamtanzahl der Elemente beträgt 52500 und die der Knoten 63726 (Riß-Modell) bzw. 53620 Elemente und 65082 Knoten (KerbModell). Die Netzweite der Modelle wurde so gewählt, daß die für die Berechnung wichtigen Stellen feiner aufgelöst werden. Neben dem Bereich oberhalb des Risses bzw. der Kerbe wurde in den Bereichen um die Initiierungsstelle die Netzweite in zwei Stufen reduziert. Direkt in der Umgebung der Rißspitze bzw. der Kerbe beträgt der Netzabstand $0,25 \mathrm{~mm}$ (auf einer Fläche von $10 \mathrm{~cm}^{2}$, d. h. für jeweils 40 Elemente). Durch die adaptive Netzverfeinerung weicht die Form der Elemente in vielen Bereichen von der eines Quaders ab. Für die Berechnungen wurde die Symmetrie des Problems in der $z$-Richtung (Abb.67, senkrecht zur Blattebene) ausgenutzt und nur die Hälfte des Modells berechnet. In der $z$-Richtung wurden 5 parallele Ebenen mit einer Netzweite von $2 \mathrm{~mm}$ erstellt. Das Projektil wurde als separates Element erstellt, so daß die Materialeigenschaften von Projektil und Probe getrennt gewählt werden können. Als Materialdaten für Gestein wurden folgende Werte, angepaßt auf die Daten von Solnhofener Kalkstein, verwendet: Elastizitätsmodul $E=62 \mathrm{GPa}$, Poissonzahl $\nu=0,3$ und die Dichte von reinem Calcit $\rho=2700 \mathrm{~kg} / \mathrm{m}^{3}$. Für Stahl wurde ein Elastizitätsmodul von $E=210 \mathrm{GPa}$, eine Poissonzahl von $\nu=0,3$ und eine Dichte von $\rho=7820 \mathrm{~kg} / \mathrm{m}^{3}$ gewählt. Diese Materialdaten wurden wahlweise für Projektil und/oder Probe verwendet, je nachdem welche Situation bei der einzelnen Berechnung nachgestellt werden sollte. Die Masse des Projektils ist für die Belastungssituation durch Wellenausbreitung unerheblich (siehe Abs. 3.3.4). Daher konnte zur Minimierung der Rechenzeit von den realen Abmessungen im Laborexperiment abgewichen werden: es wurde eine geringere Länge bezüglich der $x$-Richtung gewählt, in 

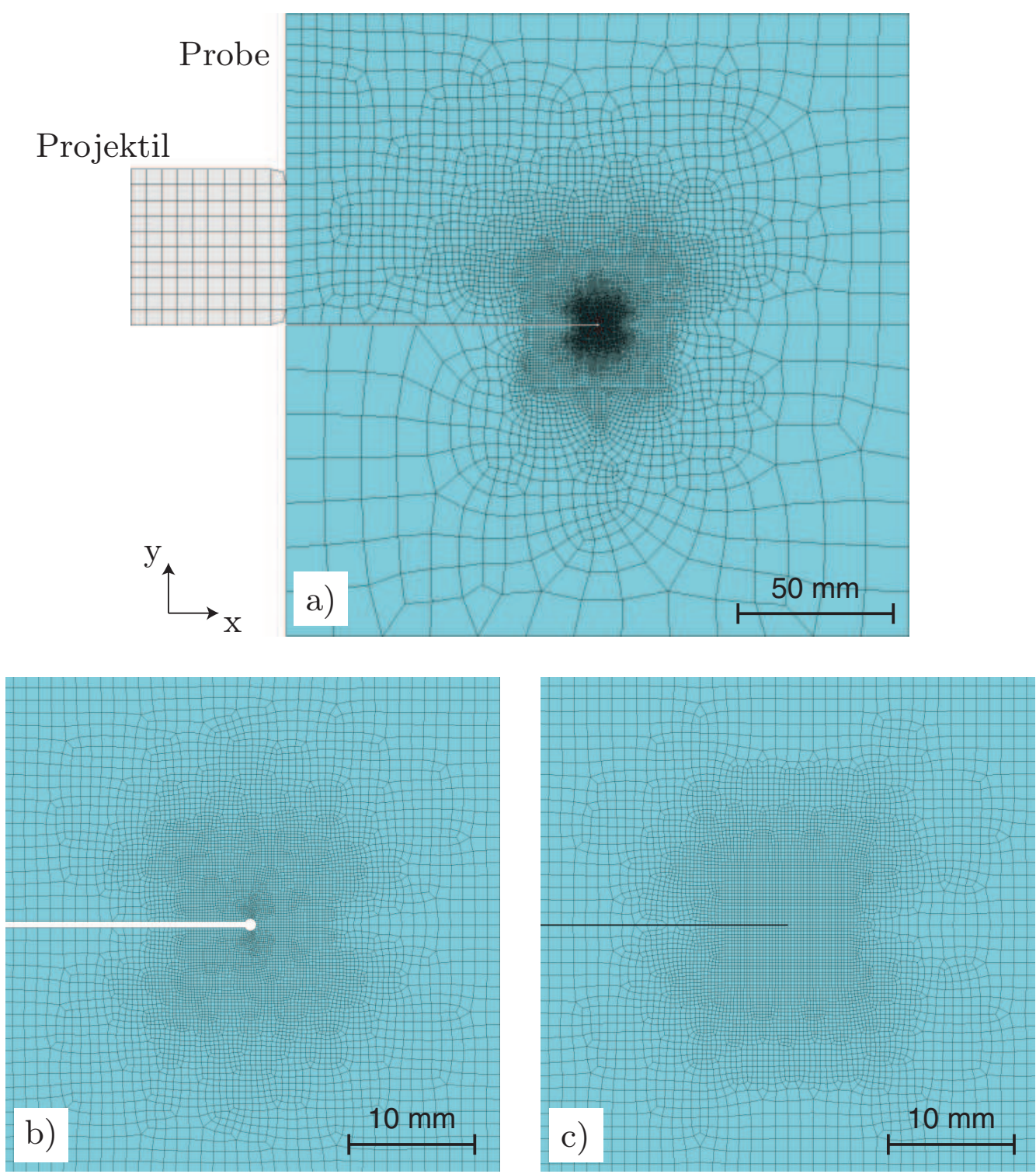

Abb. 67: Finite-Elemente-Netz: a) Gesamtansicht (äußerer Bereich für beide Modelle gleich), b) Ausschnitt um die Spitze des Kerbmodells und c) Ausschnitt um die Spitze des Rißmodells.

der $y$-Richtung stimmt die Dimensionen mit denen der realen Experimente überein und in der $z$-Richtung entspricht sie den Probenabmessungen). Bei der Wahl der Projektillänge wurde berücksichtigt, daß der Beanspruchungsvorgang an der Rißspitze nicht durch Wellenreflektionen von der Rückseite des Projektils beeinflußt wird.

Die FE-Berechnungen wurden dynamisch in 20 Zeitschritten mit einer Schrittweite $\Delta t=1,25 \mu$ s durchgeführt. Die Belastung wird dadurch simuliert, daß sich das Projektil im ersten Zeitschritt der Berechnung mit einer Geschwindigkeit $v_{0}$ in x-Richtung zu bewegen beginnt. Bilder des Belastungsvorganges sind im Haupteil der Arbeit in Abb. 31, Abs. 3.3.4 für das Kerb-Modell angegeben. Die Berechnung von bruch- 
mechanischen Kenngrößen wurde mit den in der Abaqus-Software implementierten Algorithmen durchgeführt. Dazu sei zunächst erwähnt, daß anhand des Kerb-Modells keine bruchmechanischen Kennwerte bestimmt werden können. Aufgrund der endlichen Schärfe der Kerbe ist es nicht möglich, Spannungsintensitätsfaktoren $K$ direkt zu berechnen. Auch die Bestimmung von Spannungsintensitätsfaktoren aus der spezifischen Bruchenergie $G$ mithilfe eines Contour-Integrals in der Umgebung der Kerbe führt nicht zu verwertbaren Ergebnissen. Eine solche Lösung konvergiert nicht. Für die dynamische Berechnung des Integrals über ein räumlich ausgedehntes Volumen kann, wegen Art der Belastung durch Wellen, die Beanspruchung an Kerbe nicht realistisch wiedergegeben werden. Aus diesem Grund wurden Berechnungen zur Charakterisierung der Beanspruchung lediglich mit dem Riß-Modell, d.h. für eine scharfe Rißspitze durchgeführt. Die Berechnung von Spannungsintensitätsfaktoren erfolgt dabei aus einem Contour-Integral um die Rißspitze, für das die Länge der Contour jedoch gegen Null konvergiert und somit nur Ergebnisse für die Knoten der Rißfront liefert $[212,214]$.

Ergebnisse der Berechnungen zur Untersuchung des Einflusses des Impedanzkontrastes sind in Abb. 68 in Form von Scher(Modus-II)-Spannungsintensitätsfaktoren SIF- $K_{I I}$ angegeben. Als Projektilgeschwindigkeit wurde $v_{0}=15 \mathrm{~m} / \mathrm{s}$ gewählt, für jede Materialkombination sind zwei Kurven abgebildet, eine bei Vorliegen des ebenen Dehnungszustandes für das Probeninnere und die andere für den ebenen Spannungszustand auf der Probenoberfläche. Berechnet wurde der Aufbau der Spannungsintensitätsfaktoren für wechselnde Kombinationen der Materialeigenschaften des Projektils und der Probe, diese sind: Stahlprojektil-Gesteinsprobe, GesteinsprojektilGesteinsprobe und Stahlprojektil-Stahlprobe. Der Vergleich zeigt, daß für eine Gesteinsprobe bei der Verwendung eines Stahlprojektils durch den Impedanzkontrast eine höhere Spannungsintensitätsrate $\dot{K}_{I I}$ resultiert, als für den Kontakt von Ge-

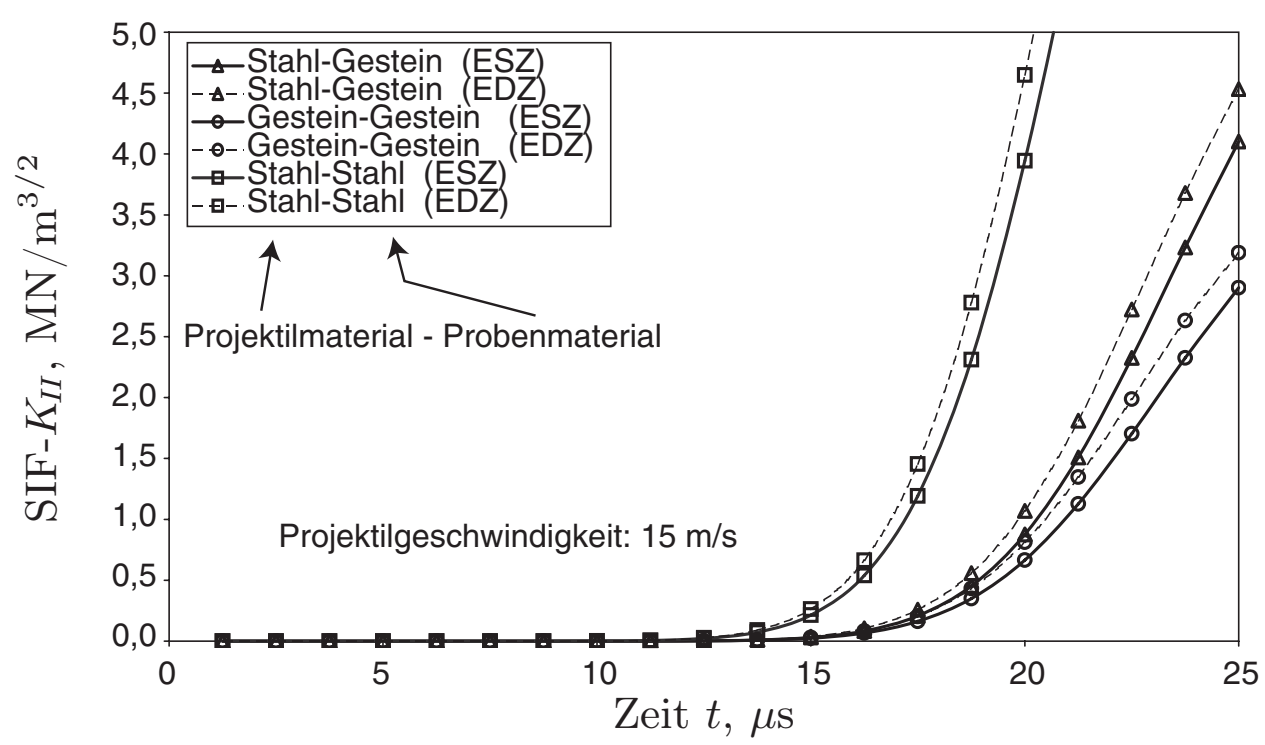

Abb. 68: Vergleich des Einflusses der Impedanz auf den Aufbau von Scher(ModusII)-Spannungsintensitätsfaktoren SIF- $K_{I I}$ im LECEI-Experiment, ermittelt durch Finite-Elemente-Modellierung. 
steinsprojektil und Gesteinsprobe. In Bezug auf die LECEI-Laborexperimente folgt daraus, daß die Verwendung eines Stahlprojektils zur Belastung einer Gesteinsprobe, ohne Einschränkungen möglich ist. Der quantitative Vergleich der Daten liefert mit Impedanzkontrast (Stahlprojektil-Gesteinsprobe) Werte für den Spannungsintensitätsfaktor $K_{I I}$, die $30 \%$ über denen ohne Impedanzkontrast liegen (GesteinsprojektilGesteinsprobe). Die Gegenüberstellung der beiden Berechnungen ohne Impedanzkontrast (Gestein-Gestein und Stahl-Stahl) illustriert die Materialabhängigkeit der Spannungsintensitätsrate $\dot{K}_{I I}$ (vergl. Abb. 32a,b in Abs. 3.3.4).

Zur Beurteilung des Einflusses der Stoßgeschwindigkeit auf den Aufbau der Spannungsintensitätsfaktoren SIF- $K_{I I}$ wurde eine weitere Serie von Berechnungen durchgeführt. In Abb. 69 sind die Ergebnisse für Projektilgeschwindigkeiten $v_{0}=10 \mathrm{~m} / \mathrm{s}$, $v_{0}=15 \mathrm{~m} / \mathrm{s}$ und $v_{0}=20 \mathrm{~m} / \mathrm{s}$ dargestellt, wiederum jeweils für den ebenen Dehnungszustand und für den ebenen Spannungszustand. Der Vergleich der Kurven ergibt, wie auch erwartet (siehe Abs. 3.3.4), einen Anstieg der Spannungsintensitätsrate $\dot{K}_{I I}$ mit der Projektilgeschwindigkeit (Stoßgeschwindigkeit). Der Vergleich der Zahlenwerte der Ergebnisse zeigt, daß dieser Zusammenhang linear ist.

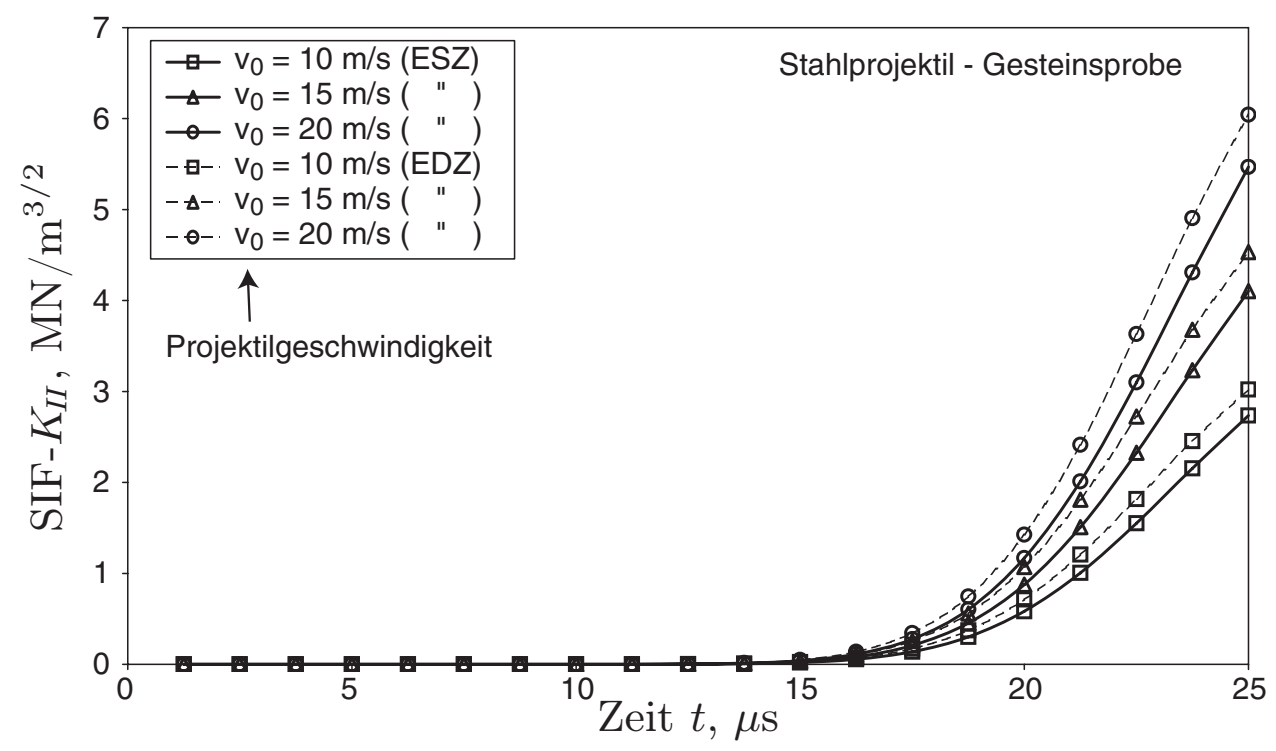

Abb. 69: Vergleich des Einflusses der Stoßgeschwindigkeit $v_{0}$ auf den Aufbau von Scher(Modus-II)-Spannungsintensitätsfaktoren SIF- $K_{I I}$ im LECEIExperiment, ermittelt durch Finite-Elemente-Modellierung. 


\section{F Ergebnistabellen}

Die in den Hauptexperimenten (siehe Abschnitt 4.4.2 und 4.5) erzielten Ergebnisse sind im Folgenden aufgelistet. Dabei werden in den Tabellen F1 und F2 zunächst die Initiierungs-Randbedingungen und dann in den Tabellen F3a-F4b die Zahlenwerte für die Rißlaufereignisse angegeben.

Die Ergebnistabellen F1 und F2 enthalten für alle getesteten Proben die Anfangsrißlänge $a_{0}$, den Kerbradius $\rho$ und die Belastungsgeschwindigkeit $v_{0}$. Die Initierungszähigkeiten, je nach Belastungsart angegeben als $K_{I c}^{s *}, K_{I d}^{*}$ bzw. $K_{I d}^{*}$, wurden von abgerundeten Kerben gewonnen (gekennzeichnet durch , ${ }^{* *}$ ), wobei die unter quasistatischer Belastung erzielten Initiierungszähigkeiten den Beginn stabiler, subkritischer Rißausbreitung (Kennzeichnung "s" $)$ wiedergeben. Die Belastungsraten $d K / d t$ wurden durch Linearisierung aus den Signalen der Rißspitzen-Dehnungsmeßstreifen bestimmt.

Tabelle F1: Experimentelle Grund- und Probendaten, Solnhofener Kalkstein

\begin{tabular}{|l||l|c|c|c|c|c|}
\hline $\begin{array}{l}\text { Probentyp \& } \\
\text { Belastungsart }\end{array}$ & $\begin{array}{l}\text { Proben- } \\
\text { nr. }\end{array}$ & $\begin{array}{c}a_{0}, \\
\mathrm{~mm}\end{array}$ & $\begin{array}{c}\rho, \\
\mathrm{mm}\end{array}$ & $\begin{array}{c}v_{0}, \\
\mathrm{~m} / \mathrm{s}\end{array}$ & $\begin{array}{c}K_{I c}^{s *}, \\
\mathrm{MN} / \mathrm{m}^{3 / 2}\end{array}$ & $\begin{array}{c}d K_{I} / d t, \\
\mathrm{MN} / \mathrm{m}^{3 / 2} / \mathrm{s}\end{array}$ \\
\hline \hline Typ I & KC01 & 10 & 0,75 & $1 \cdot 10^{-6}$ & 0,83 & $5,05 \cdot 10^{-3}$ \\
SENB & KC02 & 2,0 & 0,75 & $1 \cdot 10^{-6}$ & 0,64 & $2,60 \cdot 10^{-3}$ \\
quasistatisch & KC03 & 10 & 0,75 & $1 \cdot 10^{-6}$ & 0,79 & $4,57 \cdot 10^{-3}$ \\
& KC04 & 2,0 & 0,75 & $1 \cdot 10^{-6}$ & 0,63 & $2,75 \cdot 10^{-3}$ \\
& KD01 & 80 & 0,125 & $1 \cdot 10^{-6}$ & 0,76 & $6,49 \cdot 10^{-3}$ \\
& KD04 & 80 & 0,125 & $1 \cdot 10^{-6}$ & 0,68 & $7,27 \cdot 10^{-3}$ \\
& KD05 & 80 & 0,5 & $1 \cdot 10^{-6}$ & 0,70 & $6,67 \cdot 10^{-3}$ \\
& KD10 & 40 & 0,5 & $1 \cdot 10^{-6}$ & 1,04 & $8,33 \cdot 10^{-3}$ \\
& KD16 & 10 & 0,5 & $1 \cdot 10^{-6}$ & 1,00 & $5,22 \cdot 10^{-3}$ \\
& KE01 & 55 & 0,5 & $2 \cdot 10^{-4}$ & 1,11 & 1,03 \\
& KE02 & 60 & 0,5 & $1 \cdot 10^{-6}$ & 1,12 & $8,46 \cdot 10^{-3}$ \\
& KE03 & 70 & 0,5 & $1 \cdot 10^{-6}$ & 0,76 & $7,48 \cdot 10^{-3}$ \\
& KE04 & 80 & 0,5 & $1 \cdot 10^{-6}$ & 0,90 & $7,32 \cdot 10^{-3}$ \\
& KE05 & 50 & 0,5 & $1 \cdot 10^{-6}$ & 0,96 & $7,95 \cdot 10^{-3}$ \\
& KE06 & 40 & 0,5 & $1 \cdot 10^{-6}$ & 0,88 & $5,00 \cdot 10^{-3}$ \\
& KE07 & 30 & 0,5 & $1 \cdot 10^{-6}$ & 0,92 & $4,70 \cdot 10^{-3}$ \\
& KE08 & 20 & 0,5 & $1 \cdot 10^{-6}$ & 1,05 & $4,42 \cdot 10^{-3}$ \\
& KE09 & 10 & 0,5 & $1 \cdot 10^{-6}$ & 0,86 & $4,54 \cdot 10^{-3}$ \\
\hline \hline Probentyp \& & Proben- & $a_{0}$, & $\rho$, & $v_{0}$, & $K_{I d}^{*}$, & $d K_{I} / d t$, \\
Belastungsart & nr. & $\mathrm{mm}$ & $\mathrm{mm}$ & $\mathrm{m} / \mathrm{s}$ & $\mathrm{MN} / \mathrm{m}^{3 / 2}$ & $\mathrm{MN} / \mathrm{m}^{3 / 2} / \mathrm{s}$ \\
\hline \hline Typ I & KC05 & 10 & 0,75 & 1,0 & 1,76 & $1,02 \cdot 10^{4}$ \\
SENB & KC06 & 3,0 & 0,75 & 1,0 & 2,05 & $9,16 \cdot 10^{3}$ \\
dynamisch & KD02 & 80 & 0,125 & 1,0 & 1,38 & $1,03 \cdot 10^{4}$ \\
\hline & \multicolumn{7}{|r|}{} & \multicolumn{5}{|c|}{ Fortsetzung nächste Seite } \\
\hline
\end{tabular}


Tabelle F1: fortgesetzt

\begin{tabular}{|l||l|c|c|c|c|c|}
\hline $\begin{array}{l}\text { Probentyp \& } \\
\text { Belastungsart }\end{array}$ & $\begin{array}{l}\text { Proben- } \\
\text { nr. }\end{array}$ & $\begin{array}{c}a_{0}, \\
\mathrm{~mm}\end{array}$ & $\begin{array}{c}\rho, \\
\mathrm{mm}\end{array}$ & $\begin{array}{c}v_{0}, \\
\mathrm{~m} / \mathrm{s}\end{array}$ & $\begin{array}{c}K_{I d}^{*}, \\
\mathrm{MN} / \mathrm{m}^{3 / 2}\end{array}$ & $\begin{array}{c}d K_{I} / d t, \\
\mathrm{MN} / \mathrm{m}^{3 / 2} / \mathrm{s}\end{array}$ \\
\hline \hline Typ I & KD03 & 40 & 0,75 & 1,0 & 1,41 & $1,34 \cdot 10^{4}$ \\
SENB & KD06 & 10 & 0,75 & 2,0 & 1,15 & $1,48 \cdot 10^{4}$ \\
dynamisch & KD07 & 10 & 0,75 & 3,0 & 1,55 & $2,40 \cdot 10^{4}$ \\
& KD08 & 10 & 0,75 & 5,0 & 1,46 & $3,11 \cdot 10^{4}$ \\
& KD09 & 10 & 0,75 & 5,0 & 1,60 & $5,66 \cdot 10^{4}$ \\
& KD11 & 1,0 & 0,75 & 5,0 & - & - \\
& KD12 & 1,0 & 0,75 & 3,0 & - & - \\
\hline \hline \multirow{2}{*}{ Typ II } & & & & & $K_{I I}^{*}$, & $d K_{I I} / d t$, \\
LECEI & & & & & $\mathrm{MN} / \mathrm{m}^{3 / 2}$ & $\mathrm{MN} / \mathrm{m}^{3 / 2} / \mathrm{s}$ \\
\hline dynamisch & KD0a & 100 & 0,75 & 25 & - & - \\
& KD0b & 100 & 0,125 & 20 & - & - \\
& KD0c & 100 & 0,75 & 35 & - & $9,01 \cdot 10^{4}$ \\
& KD0e & 100 & 0,75 & 15 & 3,11 & $1,19 \cdot 10^{5}$ \\
& KD0f & 100 & 1,0 & 15 & 3,40 & $9,50 \cdot 10^{4}$ \\
& KD0g & 100 & 1,0 & 15 & 3,03 & - \\
& KD0h & 100 & 1,0 & 15 & - & - \\
& KD0i & 100 & 0,5 & 20 & - & - \\
\hline
\end{tabular}

Tabelle F2: Experimentelle Grund- und Probendaten, Arkansas Novaculit

\begin{tabular}{|c|c|c|c|c|c|c|}
\hline $\begin{array}{l}\text { Probentyp \& } \\
\text { Belastungsart }\end{array}$ & $\begin{array}{l}\text { Proben- } \\
\text { nr. }\end{array}$ & $\begin{array}{c}a_{0}, \\
\mathrm{~mm}\end{array}$ & $\begin{array}{c}\rho, \\
\mathrm{mm}\end{array}$ & $\begin{array}{c}v_{0} \\
\mathrm{~m} / \mathrm{s}\end{array}$ & $\begin{array}{c}K_{I c}^{s^{*}} \\
\mathrm{MN} / \mathrm{m}^{3 / 2}\end{array}$ & $\begin{array}{c}d K_{I} / d t, \\
\mathrm{MN} / \mathrm{m}^{3 / 2} / \mathrm{s}\end{array}$ \\
\hline $\begin{array}{l}\text { Typ A } \\
\text { SENB } \\
\text { quasistatisch }\end{array}$ & $\begin{array}{l}\text { NQC2 } \\
\text { NQB1 } \\
\text { NQD1 } \\
\text { NQG1 } \\
\text { NQC1 }\end{array}$ & $\begin{array}{l}25 \\
40 \\
20 \\
40 \\
40\end{array}$ & $\begin{array}{l}0,75 \\
0,75 \\
0,75 \\
0,75 \\
0,75\end{array}$ & $\begin{array}{l}1,0 \cdot 10^{-5} \\
1,0 \cdot 10^{-5} \\
1,0 \cdot 10^{-6} \\
1,0 \cdot 10^{-6} \\
1,0 \cdot 10^{-5}\end{array}$ & $\begin{array}{l}1,51 \\
1,55 \\
1,68 \\
1,58 \\
1,66\end{array}$ & $\begin{array}{l}1,19 \cdot 10^{-1} \\
1,33 \cdot 10^{-1} \\
1,24 \cdot 10^{-2} \\
1,29 \cdot 10^{-2} \\
1,58 \cdot 10^{-1}\end{array}$ \\
\hline $\begin{array}{l}\text { Probentyp \& } \\
\text { Belastungsart }\end{array}$ & $\begin{array}{l}\text { Proben- } \\
\text { nr. }\end{array}$ & $\begin{array}{c}a_{0}, \\
\mathrm{~mm}\end{array}$ & $\begin{array}{c}\rho, \\
\mathrm{mm}\end{array}$ & $\begin{array}{c}v_{0} \\
\mathrm{~m} / \mathrm{s}\end{array}$ & $\begin{array}{c}K_{I d}^{*}, \\
\mathrm{MN} / \mathrm{m}^{3 / 2}\end{array}$ & $\begin{array}{c}d K_{I} / d t \\
\mathrm{MN} / \mathrm{m}^{3 / 2} / \mathrm{s}\end{array}$ \\
\hline $\begin{array}{l}\text { Typ B } \\
\text { SENB } \\
\text { dynamisch }\end{array}$ & $\begin{array}{l}\text { NFC1 } \\
\text { NFB3 } \\
\text { NFB1 } \\
\text { NFD1 } \\
\text { NFB2 }\end{array}$ & $\begin{array}{l}20 \\
30 \\
40 \\
20 \\
10\end{array}$ & $\begin{array}{l}0,75 \\
0,75 \\
0,75 \\
0,75 \\
0,75\end{array}$ & $\begin{array}{l}2,0 \\
2,0 \\
2,0 \\
2,0 \\
2,0\end{array}$ & $\begin{array}{l}2,05 \\
2,92 \\
2,04 \\
2,28 \\
2,75\end{array}$ & $\begin{array}{l}2,00 \cdot 10^{4} \\
4,42 \cdot 10^{4} \\
3,49 \cdot 10^{4} \\
3,33 \cdot 10^{4} \\
3,10 \cdot 10^{4}\end{array}$ \\
\hline & \multicolumn{6}{|c|}{ Fortsetzung nächste Seite } \\
\hline
\end{tabular}


Tabelle F2: fortgesetzt

\begin{tabular}{|l||l|c|c|c|c|c|}
\hline $\begin{array}{l}\text { Probentyp \& } \\
\text { Belastungsart }\end{array}$ & $\begin{array}{l}\text { Proben- } \\
\text { nr. }\end{array}$ & $\begin{array}{c}a_{0}, \\
\mathrm{~mm}\end{array}$ & $\begin{array}{c}\rho, \\
\mathrm{mm}\end{array}$ & $\begin{array}{c}v_{0}, \\
\mathrm{~m} / \mathrm{s}\end{array}$ & $\begin{array}{c}K_{I I d}^{*}, \\
\mathrm{MN} / \mathrm{m}^{3 / 2}\end{array}$ & $\begin{array}{c}d K_{I} / d t, \\
\mathrm{MN} / \mathrm{m}^{3 / 2} / \mathrm{s}\end{array}$ \\
\hline \hline Typ C & NLG1 & 100 & 0,75 & 10 & 2,80 & $1,43 \cdot 10^{5}$ \\
LECEI & NLC1 & 100 & 0,75 & 10 & 2,55 & $1,14 \cdot 10^{5}$ \\
dynamisch & NLD1 & 100 & 0,75 & 10 & 3,00 & $1,83 \cdot 10^{5}$ \\
& NLF1 & 100 & 0,75 & 7,5 & 3,31 & $2,74 \cdot 10^{5}$ \\
& NLE1 & 100 & 0,75 & 10 & 2,09 & $1,92 \cdot 10^{5}$ \\
\hline
\end{tabular}

Sämtliche im Kapitel 4, Abschnitt 4.5 angeführten Ergebnisse sind für Solnhofener Kalkstein in den Tabellen F3a und F3b sowie für Arkansas Novaculit in den Tabellen F4a und F4b aufgelistet. Die Daten sind in Tabelle F3a bzw. F4a fortlaufend nach aufsteigenden Geschwindigkeiten sortiert. In Tabelle F3b bzw. F4b sind die Ergebnisse für die einzelnen Proben in der Reihenfolge der Applikation entlang des Rißpfades gelistet. Angegeben sind die Rißlaufgeschwindigkeit $v_{c}$, das Verhältnis der Rißlaufgeschwindigkeit zur Rayleighwellengeschwindigkeit $v_{c} / v_{R}$, die Rißlaufzähigkeit $K_{I D}$ und die spezifische Bruchausbreitungsenergie $G_{I D}$.

Tabelle F3a: Experimentelle Detaildaten, Solnhofener Kalkstein

\begin{tabular}{|c|c|c|c|c|}
\hline $\begin{array}{l}\text { Proben- } \\
\text { nr. }\end{array}$ & $\begin{array}{l}v_{c} \\
\mathrm{~m} / \mathrm{s}\end{array}$ & $\overline{v_{c} / v_{R}}$ & $\begin{array}{c}K_{I D} \\
\mathrm{MN} / \mathrm{m}^{3 / 2}\end{array}$ & $\begin{array}{r}G_{I D}, \\
\mathrm{~J} / \mathrm{m}^{2}\end{array}$ \\
\hline KD04 & $1,6 \cdot 10^{-3}$ & 0,00 & 1,27 & 23,39 \\
\hline KD01 & $1,7 \cdot 10^{-3}$ & 0,00 & 0,85 & 10,47 \\
\hline KD04 & $1,8 \cdot 10^{-3}$ & 0,00 & 1,07 & 16,59 \\
\hline KD05 & $2,3 \cdot 10^{-3}$ & 0,00 & 0,85 & 10,48 \\
\hline KD10 & $2,5 \cdot 10^{-3}$ & 0,00 & 1,09 & 17,22 \\
\hline KE03 & $3,0 \cdot 10^{-3}$ & 0,00 & 1,26 & 23,02 \\
\hline KE04 & $3,4 \cdot 10^{-3}$ & 0,00 & 1,19 & 20,54 \\
\hline KD01 & $4,5 \cdot 10^{-3}$ & 0,00 & 0,93 & 12,54 \\
\hline KD04 & $4,6 \cdot 10^{-3}$ & 0,00 & 1,22 & 21,58 \\
\hline KD10 & $6,7 \cdot 10^{-3}$ & 0,00 & 1,08 & 16,91 \\
\hline KD16 & $7,4 \cdot 10^{-3}$ & 0,00 & 1,33 & 25,65 \\
\hline KD05 & $1,5 \cdot 10^{-2}$ & 0,00 & 1,01 & 14,79 \\
\hline KD01 & $2,0 \cdot 10^{-2}$ & 0,00 & 1,05 & 15,99 \\
\hline KD10 & $3,2 \cdot 10^{-2}$ & 0,00 & 1,03 & 15,38 \\
\hline KE03 & $3,9 \cdot 10^{-2}$ & 0,00 & 1,36 & 26,82 \\
\hline KD04 & $5,5 \cdot 10^{-2}$ & 0,00 & 1,30 & 24,51 \\
\hline & \multicolumn{4}{|c|}{ Fortsetzung nächste Seite } \\
\hline
\end{tabular}


Tabelle F3a: fortgesetzt

\begin{tabular}{|l||c|c|c|c|}
\hline Proben- & $v_{c}$, & $v_{c} / v_{R}$ & $\begin{array}{c}K_{I D}, \\
\mathrm{MN} / \mathrm{m}^{3 / 2}\end{array}$ & $\begin{array}{c}G_{I D} \\
\mathrm{~J} / \mathrm{m}^{2}\end{array}$ \\
\hline \hline KE03 & $6,7 \cdot 10^{-2}$ & 0,00 & 1,38 & 27,62 \\
$\mathrm{KE04}$ & $7,5 \cdot 10^{-2}$ & 0,00 & 1,31 & 24,88 \\
KD05 & $8,8 \cdot 10^{-2}$ & 0,00 & 1,05 & 15,99 \\
KE03 & $9,3 \cdot 10^{-2}$ & 0,00 & 1,41 & 28,83 \\
KD10 & $1,2 \cdot 10^{-1}$ & 0,00 & 1,10 & 17,55 \\
KD04 & $2,0 \cdot 10^{-1}$ & 0,00 & 1,32 & 25,27 \\
KE01 & $2,0 \cdot 10^{-1}$ & 0,00 & 1,36 & 26,82 \\
KD16 & $2,0 \cdot 10^{-1}$ & 0,00 & 1,51 & 33,06 \\
KE04 & $2,5 \cdot 10^{-1}$ & 0,00 & 1,36 & 26,82 \\
KD10 & $4,4 \cdot 10^{-1}$ & 0,00 & 1,14 & 18,85 \\
KD05 & $5,2 \cdot 10^{-1}$ & 0,00 & 1,15 & 19,18 \\
KE05 & $6,2 \cdot 10^{-1}$ & 0,00 & 1,57 & 35,74 \\
KE04 & 1,02 & 0,00 & 1,44 & 30,07 \\
KE05 & 2,80 & 0,00 & 1,59 & 36,66 \\
KE05 & 7,90 & 0,00 & 1,62 & 38,06 \\
KE06 & 16,0 & 0,01 & 1,57 & 35,74 \\
KD16 & 28,7 & 0,01 & 1,56 & 35,29 \\
KE07 & 77,0 & 0,03 & 1,66 & 39,98 \\
KE07 & 93,0 & 0,03 & 1,66 & 39,99 \\
KE07 & 124 & 0,04 & 1,69 & 41,47 \\
KD16 & 270 & 0,10 & 1,55 & 35,05 \\
KC03 & 286 & 0,10 & 1,70 & 42,19 \\
KE08 & 287 & 0,10 & 1,65 & 39,75 \\
KE09 & 346 & 0,12 & 1,75 & 44,85 \\
KE08 & 347 & 0,12 & 1,71 & 42,82 \\
KD16 & 366 & 0,13 & 1,54 & 34,77 \\
KE08 & 366 & 0,13 & 1,71 & 42,87 \\
KC01 & 367 & 0,13 & 1,60 & 37,54 \\
KE09 & 374 & 0,13 & 1,74 & 44,41 \\
KE09 & 392 & 0,14 & 1,70 & 42,44 \\
KD02 & 460 & 0,16 & 1,77 & 46,23 \\
KD02 & 654 & 0,23 & 1,88 & 53,13 \\
KC04 & 694 & 0,25 & 2,09 & 65,97 \\
KC02 & 703 & 0,25 & 1,53 & 35,39 \\
KD05 & 960 & 0,34 & 1,76 & 48,69 \\
\hline & 980 & 0,35 & 1,77 & 49,42 \\
KD09 & 1120 & 0,40 & 1,72 & 48,02 \\
\hline
\end{tabular}


Tabelle F3a: fortgesetzt

\begin{tabular}{|l||c|c|c|r|}
\hline $\begin{array}{l}\text { Proben- } \\
\text { nr. }\end{array}$ & $\begin{array}{c}v_{c}, \\
\mathrm{~m} / \mathrm{s}\end{array}$ & $v_{c} / v_{R}$ & $\begin{array}{c}K_{I D}, \\
\mathrm{MN} / \mathrm{m}^{3 / 2}\end{array}$ & $\begin{array}{r}G_{I D} \\
\mathrm{~J} / \mathrm{m}^{2}\end{array}$ \\
\hline \hline KD07 & 1195 & 0,43 & 2,00 & 66,07 \\
KD09 & 1290 & 0,46 & 1,93 & 63,07 \\
KD08 & 1330 & 0,48 & 1,77 & 53,65 \\
KD09 & 1380 & 0,49 & 2,08 & 75,21 \\
KC06 & 1410 & 0,50 & 2,05 & 73,75 \\
KD0g & 1440 & 0,51 & 2,11 & 78,90 \\
KD0e & 1440 & 0,51 & 2,33 & 96,21 \\
KD0e & 1440 & 0,51 & 2,49 & 109,87 \\
KD09 & 1495 & 0,53 & 2,17 & 85,05 \\
KD08 & 1500 & 0,54 & 2,04 & 75,30 \\
KD0f & 1550 & 0,55 & 1,72 & 54,53 \\
KD0g & 1570 & 0,56 & 2,28 & 96,57 \\
KD0g & 1580 & 0,56 & 2,39 & 106,53 \\
KD0f & 1585 & 0,57 & 2,31 & 99,72 \\
KD0e & 1590 & 0,57 & 2,76 & 142,65 \\
KD0f & 1620 & 0,58 & 3,75 & 266,61 \\
KD06 & 1623 & 0,58 & 2,02 & 77,46 \\
KD07 & 1630 & 0,58 & 2,29 & 99,85 \\
KD0e & 1640 & 0,59 & 3,04 & 176,71 \\
KD08 & 1650 & 0,59 & 2,07 & 82,29 \\
KD08 & 1670 & 0,60 & 2,16 & 90,40 \\
KD07 & 1680 & 0,60 & 2,42 & 113,99 \\
KD09 & 1700 & 0,61 & 2,34 & 107,57 \\
KD0g & 1700 & 0,61 & 2,64 & 136,92 \\
KD0h & 1760 & 0,63 & 2,63 & 139,94 \\
KD11 & 1815 & 0,65 & 2,23 & 103,59 \\
KD0lb & 1820 & 0,65 & 2,86 & 170,86 \\
KD0h & 1840 & 0,66 & 3,67 & 284,55 \\
KD12 & 1848 & 0,66 & 2,67 & 151,31 \\
KD0la & 1850 & 0,66 & 3,15 & 210,84 \\
KD0f & 1880 & 0,67 & 3,86 & 322,32 \\
\hline
\end{tabular}


Tabelle F3b: Experimentelle Detaildaten, Solnhofener Kalkstein

\begin{tabular}{|c|c|c|c|c|c|}
\hline $\begin{array}{l}\text { Probentyp \& } \\
\text { Belastungsart }\end{array}$ & $\begin{array}{l}\text { Proben- } \\
\text { nr. }\end{array}$ & $\begin{array}{c}v_{c} \\
\mathrm{~m} / \mathrm{s}\end{array}$ & $v_{c} / v_{R}$ & $\begin{array}{c}K_{I D}, \\
\mathrm{MN} / \mathrm{m}^{3 / 2} \\
\end{array}$ & $\begin{array}{r}G_{I D}, \\
\mathrm{~J} / \mathrm{m}^{2}\end{array}$ \\
\hline \multirow{36}{*}{$\begin{array}{r}\text { Typ I } \\
\text { SENB } \\
\text { quasistatisch }\end{array}$} & $\mathrm{KC} 01$ & 367 & 0,13 & 1,60 & 37,54 \\
\hline & KC02 & 703 & 0,25 & 1,53 & 35,39 \\
\hline & KC03 & 286 & 0,10 & 1,70 & 42,19 \\
\hline & $\mathrm{KC04}$ & 694 & 0,25 & 2,09 & 65,97 \\
\hline & KD01 & $1,7 \cdot 10^{-3}$ & 0,00 & 0,85 & 10,47 \\
\hline & KD01 & $4,5 \cdot 10^{-3}$ & 0,00 & 0,93 & 12,54 \\
\hline & KD01 & $2,0 \cdot 10^{-2}$ & 0,00 & 1,05 & 15,99 \\
\hline & KD04 & $1,60 \cdot 10^{-3}$ & 0,00 & 1,27 & 23,39 \\
\hline & KD04 & $1,80 \cdot 10^{-3}$ & 0,00 & 1,07 & 16,59 \\
\hline & KD04 & $4,60 \cdot 10^{-3}$ & 0,00 & 1,22 & 21,58 \\
\hline & KD04 & $5,50 \cdot 10^{-2}$ & 0,00 & 1,30 & 24,51 \\
\hline & KD04 & $2,00 \cdot 10^{-1}$ & 0,00 & 1,32 & 25,27 \\
\hline & KD05 & $2,30 \cdot 10^{-3}$ & 0,00 & 0,85 & 10,48 \\
\hline & KD05 & $1,50 \cdot 10^{-2}$ & 0,00 & 1,01 & 14,79 \\
\hline & KD05 & $8,80 \cdot 10^{-2}$ & 0,00 & 1,05 & 15,99 \\
\hline & KD05 & $5,20 \cdot 10^{-1}$ & 0,00 & 1,15 & 19,18 \\
\hline & KD10 & $2,5 \cdot 10^{-3}$ & 0,00 & 1,09 & 17,22 \\
\hline & KD10 & $6,7 \cdot 10^{-3}$ & 0,00 & 1,08 & 16,91 \\
\hline & KD10 & $3,2 \cdot 10^{-2}$ & 0,00 & 1,03 & 15,38 \\
\hline & KD10 & $1,2 \cdot 10^{-1}$ & 0,00 & 1,10 & 17,55 \\
\hline & KD10 & $4,4 \cdot 10^{-1}$ & 0,00 & 1,14 & 18,85 \\
\hline & KD16 & $7,40 \cdot 10^{-3}$ & 0,00 & 1,33 & 25,65 \\
\hline & KD16 & $2,00 \cdot 10^{-1}$ & 0,00 & 1,51 & 33,06 \\
\hline & KD16 & 28,7 & 0,01 & 1,56 & 35,29 \\
\hline & KD16 & 270 & 0,10 & 1,55 & 35,05 \\
\hline & KD16 & 366 & 0,13 & 1,54 & 34,77 \\
\hline & KE01 & $2,00 \cdot 10^{-1}$ & 0,00 & 1,36 & 26,82 \\
\hline & KE03 & $3,00 \cdot 10^{-3}$ & 0,00 & 1,26 & 23,02 \\
\hline & KE03 & $3,90 \cdot 10^{-2}$ & 0,00 & 1,36 & 26,82 \\
\hline & KE03 & $6,70 \cdot 10^{-2}$ & 0,00 & 1,38 & 27,62 \\
\hline & KE03 & $9,30 \cdot 10^{-2}$ & 0,00 & 1,41 & 28,83 \\
\hline & KE04 & $3,40 \cdot 10^{-3}$ & 0,00 & 1,19 & 20,54 \\
\hline & KE04 & $7,50 \cdot 10^{-2}$ & 0,00 & 1,31 & 24,88 \\
\hline & KE04 & $2,50 \cdot 10^{-1}$ & 0,00 & 1,36 & 26,82 \\
\hline & KE04 & 1,02 & 0,00 & 1,44 & 30,07 \\
\hline & KE05 & $6,20 \cdot 10^{-1}$ & 0,00 & 1,57 & 35,74 \\
\hline & & & For & etzung näc & Seite \\
\hline
\end{tabular}


Tabelle F3b: fortgesetzt

\begin{tabular}{|c|c|c|c|c|c|}
\hline $\begin{array}{l}\text { Probentyp \& } \\
\text { Belastungsart }\end{array}$ & $\begin{array}{l}\text { Proben- } \\
\text { nr. }\end{array}$ & $\begin{array}{l}v_{c} \\
\mathrm{~m} / \mathrm{s}\end{array}$ & $v_{c} / v_{R}$ & $\begin{array}{c}K_{I D} \\
\mathrm{MN} / \mathrm{m}^{3 / 2}\end{array}$ & $\begin{array}{r}G_{I D}, \\
\mathrm{~J} / \mathrm{m}^{2} \\
\end{array}$ \\
\hline \multirow{12}{*}{$\begin{array}{r}\text { Typ I } \\
\text { SENB } \\
\text { quasistatisch }\end{array}$} & KE05 & 2,8 & 0,00 & 1,59 & 36,66 \\
\hline & KE05 & 7,9 & 0,00 & 1,62 & 38,06 \\
\hline & KE06 & 16 & 0,01 & 1,57 & 35,74 \\
\hline & KE07 & 77 & 0,03 & 1,66 & 39,98 \\
\hline & KE07 & 93 & 0,03 & 1,66 & 39,99 \\
\hline & KE07 & 124 & 0,04 & 1,69 & 41,47 \\
\hline & KE08 & 287 & 0,10 & 1,65 & 39,75 \\
\hline & KE08 & 347 & 0,12 & 1,71 & 42,82 \\
\hline & KE08 & 366 & 0,13 & 1,71 & 42,87 \\
\hline & KE09 & 346 & 0,12 & 1,75 & 44,85 \\
\hline & KE09 & 374 & 0,13 & 1,74 & 44,41 \\
\hline & KE09 & 392 & 0,14 & 1,70 & 42,44 \\
\hline \multirow{20}{*}{$\begin{array}{r}\text { Typ I } \\
\text { SENB } \\
\text { dynamisch }\end{array}$} & KC05 & 960 & 0,34 & 1,76 & 48,69 \\
\hline & KC06 & 1410 & 0,50 & 2,05 & 73,75 \\
\hline & KD02 & 460 & 0,16 & 1,77 & 46,23 \\
\hline & KD02 & 654 & 0,23 & 1,88 & 53,13 \\
\hline & KD03 & 980 & 0,35 & 1,77 & 49,42 \\
\hline & KD06 & 1623 & 0,58 & 2,02 & 77,46 \\
\hline & KD07 & 1195 & 0,43 & 2,00 & 66,07 \\
\hline & KD07 & 1630 & 0,58 & 2,29 & 99,85 \\
\hline & KD07 & 1680 & 0,60 & 2,42 & 113,99 \\
\hline & KD08 & 1330 & 0,48 & 1,77 & 53,65 \\
\hline & KD08 & 1500 & 0,54 & 2,04 & 75,30 \\
\hline & KD08 & 1650 & 0,59 & 2,07 & 82,29 \\
\hline & KD08 & 1670 & 0,60 & 2,16 & 90,40 \\
\hline & KD09 & 1120 & 0,40 & 1,72 & 48,02 \\
\hline & KD09 & 1290 & 0,46 & 1,93 & 63,07 \\
\hline & KD09 & 1380 & 0,49 & 2,08 & 75,21 \\
\hline & KD09 & 1495 & 0,53 & 2,17 & 85,05 \\
\hline & KD09 & 1700 & 0,61 & 2,34 & 107,57 \\
\hline & KD11 & 1815 & 0,65 & 2,23 & 103,59 \\
\hline & KD12 & 1848 & 0,66 & 2,67 & 151,31 \\
\hline \multirow{4}{*}{$\begin{array}{r}\text { Typ II } \\
\text { LECEI } \\
\text { dynamisch }\end{array}$} & KD0e & 1440 & 0,51 & 2,33 & 96,21 \\
\hline & KD0e & 1440 & 0,51 & 2,49 & 109,87 \\
\hline & KD0e & 1590 & 0,57 & 2,76 & 142,65 \\
\hline & KD0e & 1640 & 0,59 & 3,04 & 176,71 \\
\hline
\end{tabular}


Tabelle F3b: fortgesetzt

\begin{tabular}{|r|l||c|c|c|r|}
\hline $\begin{array}{r}\text { Probentyp \& } \\
\text { Belastungsart }\end{array}$ & Proben- & $\begin{array}{c}v_{c}, \\
\mathrm{~m} / \mathrm{s} .\end{array}$ & $v_{c} / v_{R}$ & $\begin{array}{c}K_{I D}, \\
\mathrm{MN} / \mathrm{m}^{3 / 2}\end{array}$ & $\begin{array}{r}G_{I D}, \\
\mathrm{~J} / \mathrm{m}^{2}\end{array}$ \\
\hline \hline Typ II & KD0f & 1550 & 0,55 & 1,72 & 54,53 \\
LECEI & KD0f & 1585 & 0,57 & 2,31 & 99,72 \\
dynamisch & KD0f & 1620 & 0,58 & 3,75 & 266,61 \\
& KD0f & 1880 & 0,67 & 3,86 & 322,32 \\
\cline { 2 - 6 } & KD0g & 1440 & 0,51 & 2,11 & 78,90 \\
& KD0g & 1570 & 0,56 & 2,28 & 96,57 \\
& KD0g & 1580 & 0,56 & 2,39 & 106,53 \\
& KD0g & 1700 & 0,61 & 2,64 & 136,92 \\
\cline { 2 - 6 } & KD0h & 1760 & 0,63 & 2,63 & 139,94 \\
& KD0h & 1840 & 0,66 & 3,67 & 284,55 \\
\cline { 2 - 6 } & KD0la & 1850 & 0,66 & 3,15 & 210,84 \\
\cline { 2 - 6 } & KD0lb & 1820 & 0,65 & 2,86 & 170,86 \\
\hline
\end{tabular}

Tabelle F4a: Experimentelle Detaildaten, Arkansas Novaculit

\begin{tabular}{|l||c|c|c|c|}
\hline $\begin{array}{l}\text { Proben- } \\
\text { nr. }\end{array}$ & $\begin{array}{c}v_{c}, \\
\mathrm{~m} / \mathrm{s}\end{array}$ & $v_{c} / v_{R}$ & $\begin{array}{c}K_{I D}, \\
\mathrm{MN} / \mathrm{m}^{3 / 2}\end{array}$ & $\begin{array}{c}G_{I D}, \\
\mathrm{~J} / \mathrm{m}^{2}\end{array}$ \\
\hline \hline NQD1 & $1,4 \cdot 10^{-4}$ & 0,00 & 2,40 & 63,90 \\
NQD1 & $6,4 \cdot 10^{-4}$ & 0,00 & 3,00 & 103,04 \\
NQC1 & $2,5 \cdot 10^{-3}$ & 0,00 & 2,77 & 87,04 \\
NQB1 & $2,8 \cdot 10^{-3}$ & 0,00 & 2,06 & 48,13 \\
NQC2 & $3,8 \cdot 10^{-3}$ & 0,00 & 2,50 & 70,90 \\
NQD1 & $4,0 \cdot 10^{-3}$ & 0,00 & 3,00 & 102,06 \\
NQC2 & $2,5 \cdot 10^{-2}$ & 0,00 & 2,60 & 76,68 \\
NQC1 & $1,0 \cdot 10^{-1}$ & 0,00 & 3,01 & 102,77 \\
NQB1 & $1,2 \cdot 10^{-1}$ & 0,00 & 2,60 & 76,68 \\
NQC1 & 1,20 & 0,00 & 2,95 & 98,72 \\
NQB1 & 2,38 & 0,00 & 2,70 & 82,69 \\
NQC2 & 32,0 & 0,01 & 3,00 & 102,10 \\
NFC1 & 189 & 0,06 & 3,17 & 114,22 \\
NQC1 & 207 & 0,06 & 2,97 & 100,31 \\
NQD1 & 310 & 0,09 & 3,00 & 102,65 \\
NQD1 & 340 & 0,10 & 3,00 & 102,77 \\
NFC1 & 611 & 0,19 & 2,96 & 101,56 \\
NFB3 & 809 & 0,25 & 3,37 & 133,89 \\
NFC1 & 861 & 0,26 & 3,61 & 154,45 \\
\hline \multicolumn{4}{||r|}{ Fortsetzung nächste Seite } \\
\hline
\end{tabular}


Tabelle F4a: fortgesetzt

\begin{tabular}{|c|c|c|c|c|}
\hline $\begin{array}{l}\text { Proben- } \\
\text { nr. }\end{array}$ & $\begin{array}{l}v_{c} \\
\mathrm{~m} / \mathrm{s}\end{array}$ & $v_{c} / v_{R}$ & $\begin{array}{c}K_{I D} \\
\mathrm{MN} / \mathrm{m}^{3 / 2}\end{array}$ & $\begin{array}{l}G_{I D} \\
\mathrm{~J} / \mathrm{m}^{2}\end{array}$ \\
\hline NFB1 & 945 & 0,29 & 3,32 & 131,88 \\
\hline NFD1 & 949 & 0,29 & 3,68 & 162,11 \\
\hline NFC1 & 1045 & 0,32 & 3,01 & 109,78 \\
\hline NFB3 & 1096 & 0,33 & 3,44 & 144,38 \\
\hline NLE1 & 1132 & 0,34 & 3,19 & 124,81 \\
\hline NLE1 & 1200 & 0,36 & 4,11 & 209,36 \\
\hline NFB1 & 1208 & 0,37 & 3,16 & 123,92 \\
\hline NFC1 & 1231 & 0,37 & 4,13 & 212,48 \\
\hline NFB2 & 1244 & 0,38 & 3,59 & 160,89 \\
\hline NFD1 & 1266 & 0,38 & 3,48 & 151,75 \\
\hline NLG1 & 1300 & 0,39 & 3,40 & 145,72 \\
\hline NLG1 & 1300 & 0,39 & 3,80 & 182,02 \\
\hline NFB3 & 1347 & 0,41 & 3,59 & 163,88 \\
\hline NFD1 & 1364 & 0,41 & 3,89 & 193,02 \\
\hline NFB2 & 1404 & 0,43 & 3,82 & 187,58 \\
\hline NFB3 & 1456 & 0,44 & 3,56 & 164,65 \\
\hline NLD1 & 1470 & 0,45 & 3,58 & 167,02 \\
\hline NLF1 & 1490 & 0,45 & 4,10 & 220,01 \\
\hline NFD1 & 1566 & 0,47 & 4,14 & 228,22 \\
\hline NFB3 & 1672 & 0,51 & 4,18 & 238,91 \\
\hline NFD1 & 1674 & 0,51 & 3,84 & 201,74 \\
\hline NLF1 & 1720 & 0,52 & 4,02 & 223,87 \\
\hline NLD1 & 1750 & 0,53 & 6,26 & 547,50 \\
\hline NFB2 & 1763 & 0,53 & 4,83 & 327,14 \\
\hline NFB2 & 1780 & 0,54 & 4,25 & 254,58 \\
\hline NLC1 & 1860 & 0,56 & 5,00 & 361,19 \\
\hline NFB2 & 1934 & 0,59 & 4,29 & 272,59 \\
\hline NLF1 & 1950 & 0,59 & 5,33 & 423,18 \\
\hline NLF1 & 2000 & 0,61 & 5,41 & 444,06 \\
\hline NLC1 & 2090 & 0,63 & 7,90 & 981,41 \\
\hline NLE1 & 2097 & 0,64 & 5,15 & 418,30 \\
\hline NLE1 & 2158 & 0,65 & 7,29 & 860,74 \\
\hline NLE1 & 2175 & 0,66 & 6,37 & 662,53 \\
\hline NLD1 & 2210 & 0,67 & 6,51 & 703,50 \\
\hline NLF1 & 2250 & 0,68 & 5,66 & 542,48 \\
\hline NLD1 & 2290 & 0,69 & 6,88 & 818,51 \\
\hline NLC1 & 2330 & 0,71 & 7,07 & 883,65 \\
\hline \multicolumn{5}{|c|}{ Fortsetzung nächste Seite } \\
\hline
\end{tabular}


Tabelle F4a: fortgesetzt

\begin{tabular}{|l||c|c|c|c|}
\hline $\begin{array}{l}\text { Proben- } \\
\text { nr. }\end{array}$ & $\begin{array}{c}v_{c}, \\
\mathrm{~m} / \mathrm{s}\end{array}$ & $v_{c} / v_{R}$ & $\begin{array}{c}K_{I D}, \\
\mathrm{MN} / \mathrm{m}^{3 / 2}\end{array}$ & $\begin{array}{c}G_{I D}, \\
\mathrm{~J} / \mathrm{m}^{2}\end{array}$ \\
\hline \hline NLC1 & 2340 & 0,71 & 7,09 & 893,75 \\
NLD1 & 2360 & 0,72 & 7,30 & 958,59 \\
NLC1 & 2400 & 0,73 & 7,44 & 1020,23 \\
\hline
\end{tabular}

Tabelle F4b: Experimentelle Detaildaten, Arkansas Novaculit

\begin{tabular}{|r|l||c|c|c|c|}
\hline $\begin{array}{r}\text { Probentyp \& } \\
\text { Belastungsart }\end{array}$ & Proben- & $\begin{array}{c}v_{c}, \\
\mathrm{mr} / \mathrm{s}\end{array}$ & $v_{c} / v_{R}$ & $\begin{array}{c}K_{I D}, \\
\mathrm{MN} / \mathrm{m}^{3 / 2}\end{array}$ & $\begin{array}{c}G_{I D}, \\
\mathrm{~J} / \mathrm{m}^{2}\end{array}$ \\
\hline \hline Typ A & NQB1 & $2,8 \cdot 10^{-3}$ & 0,00 & 2,06 & 48,13 \\
SENB & NQB1 & $1,2 \cdot 10^{-1}$ & 0,00 & 2,60 & 76,68 \\
quasistatisch & NQB1 & 2,38 & 0,00 & 2,70 & 82,69 \\
\cline { 2 - 6 } & NQC1 & $2,5 \cdot 10^{-3}$ & 0,00 & 2,77 & 87,04 \\
& NQC1 & $1,0 \cdot 10^{-1}$ & 0,00 & 3,01 & 102,77 \\
& NQC1 & 1,20 & 0,00 & 2,95 & 98,72 \\
& NQC1 & 207 & 0,06 & 2,97 & 100,31 \\
\cline { 2 - 6 } & NQC2 & $2,5 \cdot 10^{-2}$ & 0,00 & 2,60 & 76,68 \\
& NQC2 & $3,8 \cdot 10^{-3}$ & 0,00 & 2,50 & 70,90 \\
& NQC2 & 32,0 & 0,01 & 3,00 & 102,10 \\
\cline { 2 - 6 } & NQD1 & $1,4 \cdot 10^{-4}$ & 0,00 & 2,40 & 63,90 \\
& NQD1 & 310 & 0,09 & 3,00 & 102,65 \\
& NQD1 & 340 & 0,10 & 3,00 & 102,77 \\
& NQD1 & $4,0 \cdot 10^{-3}$ & 0,00 & 3,00 & 102,06 \\
& NQD1 & $6,4 \cdot 10^{-4}$ & 0,00 & 3,00 & 103,04 \\
\hline \multirow{7}{*}{ Typ B } & NFB1 & 1208 & 0,37 & 3,16 & 123,92 \\
SENB & NFB1 & 945 & 0,29 & 3,32 & 131,88 \\
\cline { 2 - 6 } dynamisch & NFB2 & 1244 & 0,38 & 3,59 & 160,89 \\
& NFB2 & 1404 & 0,43 & 3,82 & 187,58 \\
& NFB2 & 1763 & 0,53 & 4,83 & 327,14 \\
& NFB2 & 1780 & 0,54 & 4,25 & 254,58 \\
& NFB2 & 1934 & 0,59 & 4,29 & 272,59 \\
\cline { 2 - 6 } & NFB3 & 1096 & 0,33 & 3,44 & 144,38 \\
& NFB3 & 1347 & 0,41 & 3,59 & 163,88 \\
& NFB3 & 1456 & 0,44 & 3,56 & 164,65 \\
& NFB3 & $1672^{2}$ & 0,51 & 4,18 & 238,91 \\
& NFB3 & 809 & 0,25 & 3,37 & 133,89 \\
\hline & & $F o r t s e t z u n g ~ n \ddot{c h s t e ~ S e i t e ~}$ \\
\hline
\end{tabular}


Tabelle F4b: fortgesetzt

\begin{tabular}{|r|l||c|c|c|c|}
\hline $\begin{array}{r}\text { Probentyp \& } \\
\text { Belastungsart }\end{array}$ & Proben- & $\begin{array}{c}v_{c}, \\
\mathrm{~m} / \mathrm{s}\end{array}$ & $v_{c} / v_{R}$ & $\begin{array}{c}K_{I D}, \\
\mathrm{MN} / \mathrm{m}^{3 / 2}\end{array}$ & $\begin{array}{c}G_{I D}, \\
\mathrm{~J} / \mathrm{m}^{2}\end{array}$ \\
\hline \hline Typ B & NFC1 & 1045 & 0,32 & 3,01 & 109,78 \\
SENB & NFC1 & 1231 & 0,37 & 4,13 & 212,48 \\
dynamisch & NFC1 & 189 & 0,06 & 3,17 & 114,22 \\
& NFC1 & 611 & 0,19 & 2,96 & 101,56 \\
& NFC1 & 861 & 0,26 & 3,61 & 154,45 \\
\cline { 2 - 6 } & NFD1 & 1266 & 0,38 & 3,48 & 151,75 \\
& NFD1 & 1364 & 0,41 & 3,89 & 193,02 \\
& NFD1 & 1566 & 0,47 & 4,14 & 228,22 \\
& NFD1 & 1674 & 0,51 & 3,84 & 201,74 \\
& NFD1 & 949 & 0,29 & 3,68 & 162,11 \\
\hline TYP C & NLC1 & 1860 & 0,56 & 5,00 & 361,19 \\
LECEI & NLC1 & 2090 & 0,63 & 7,90 & 981,41 \\
& NLC1 & 2330 & 0,71 & 7,07 & 883,65 \\
& NLC1 & 2340 & 0,71 & 7,09 & 893,75 \\
& NLC1 & 2400 & 0,73 & 7,44 & 1020,23 \\
\cline { 2 - 6 } & NLD1 & 1470 & 0,45 & 3,58 & 167,02 \\
& NLD1 & 1750 & 0,53 & 6,26 & 547,50 \\
& NLD1 & 2210 & 0,67 & 6,51 & 703,50 \\
& NLD1 & 2290 & 0,69 & 6,88 & 818,51 \\
& NLD1 & 2360 & 0,72 & 7,30 & 958,59 \\
\cline { 2 - 6 } & NLE1 & 1132 & 0,34 & 3,19 & 124,81 \\
& NLE1 & 1200 & 0,36 & 4,11 & 209,36 \\
& NLE1 & 2097 & 0,64 & 5,15 & 418,30 \\
& NLE1 & 2158 & 0,65 & 7,29 & 860,74 \\
& NLE1 & 2175 & 0,66 & 6,37 & 662,53 \\
\cline { 2 - 6 } & NLF1 & 1490 & 0,45 & 4,10 & 220,01 \\
& NLF1 & 1720 & 0,52 & 4,02 & 223,87 \\
& NLF1 & 1950 & 0,59 & 5,33 & 423,18 \\
& NLF1 & 2000 & 0,61 & 5,41 & 444,06 \\
& NLF1 & 2250 & 0,68 & 5,66 & 542,48 \\
\cline { 2 - 6 } & NLG1 & 1300 & 0,39 & 3,40 & 145,72 \\
& NLG1 & 1300 & 0,39 & 3,80 & 182,02 \\
\hline
\end{tabular}


